\title{
Limits on Planetary Companions from Doppler Surveys of Nearby Stars $^{1}$
}

\author{
Andrew W. Howard, Benjamin J. Fulton \\ Institute for Astronomy, University of Hawaii, 2680 Woodlawn Drive, Honolulu, HI 96822, \\ USA
}

\begin{abstract}
Most of our knowledge of planets orbiting nearby stars comes from Doppler surveys. For spaced-based, high-contrast imaging missions, nearby stars with Doppler-discovered planets are attractive targets. The known orbits tell imaging missions where and when to observe, and the dynamically-determined masses provide important constraints for the interpretation of planetary spectra. Quantifying the set of planet masses and orbits that could have been detected will enable more efficient planet discovery and characterization. We analyzed Doppler measurements from Lick and Keck Observatories collected by the California Planet Survey. We focused on stars that are likely targets for three space-based planet imaging mission concepts studied by NASA - WFIRST-AFTA, Exo-C, and Exo$\mathrm{S}$. The Doppler targets are primarily F8 and later main sequence stars, with observations spanning 1987-2014. We identified 76 stars with Doppler measurements from the prospective mission target lists. We developed an automated planet search and a methodology to estimate the pipeline completeness using injection and recovery tests. We applied this machinery to the Doppler data and computed planet detection limits for each star as a function of planet minimum mass and semi-major axis. For typical stars in the survey, we are sensitive to approximately Saturn-mass planets inside of 1 AU, Jupiter-mass planets inside of $\sim 3 \mathrm{AU}$, and our sensitivity declines out to $\sim 10 \mathrm{AU}$. For the best Doppler targets, we are sensitive to Neptune-mass planets in 3 AU orbits. Using an idealized model of Doppler survey completeness, we forecast the precision of future surveys of non-ideal Doppler targets that are likely targets of imaging missions.
\end{abstract}

Subject headings: Extrasolar Planets — Data Analysis and Techniques

\footnotetext{
${ }^{1}$ Based on observations obtained at the W. M. Keck Observatory, which is operated jointly by the University of California and the California Institute of Technology. Keck time has been granted by NASA, the University of California, and the University of Hawaii.
} 


\section{Introduction}

NASA recently studied three mission concepts capable of directly imaging extrasolar planets from space. The WFIRST-AFTA mission is envisioned as a wide-field, infrared imager on a 2.4-m telescope that could accomplish broad astrophysical goals (Spergel et al. 2013). The baseline mission includes a coronagraph in the instrument suite for exoplanet detection and characterization (Traub et al. 2014; Goullioud et al. 2014). In addition, two Science and Technology Definition Teams (STDTs) studied probe-scale (cost less than \$1B) mission concepts for the direct detection of extrasolar planets orbiting nearby stars. Exo$\mathrm{C}$ is a mission concept based on a telescope with an internal coronagraph to generate the ultra-high contrast images needed for planet detection (Stapelfeldt et al. 2014). Exo-S would image extrasolar planets using a pair of spacecraft flying in formation - an external occulter (starshade) and a telescope (Seager et al. 2014).

Teams studying these possible missions are considering the scientific return of each, including the discovery of new exoplanets orbiting nearby stars, spectroscopy of exoplanet atmospheres, and imaging of debris and protoplanetary disks. Each mission needs a list of nearby stars to search for new planets and to characterize existing planetary systems. The characteristics of these stars (brightness, spectral type, distance, age, sky position, multiplicity, etc.) make some more favorable targets than others. Target selection will also be based on which stars have planets and debris disks already known. Most of our knowledge of exoplanets orbiting nearby stars comes from Doppler surveys that started in the late 1980s and have improved in sensitivity since then. These observational surveys have discovered hundreds of planets orbiting nearby stars, some of which are possible targets for Exo-C, Exo-S, and WFIRST-AFTA. Many of the Doppler target stars have clear non-detections of planets after hundreds of measurements spanning a decade or more. These non-detections can be quantified, as we do below, using injection-recovery tests to measure planet search completeness as a function of planet minimum mass $\left(M_{\mathrm{p}} \sin i\right)$ and orbital period (with assumptions about eccentricities). Knowing that certain stars lack planets with particular masses and semi-major axes constitutes useful information for the imaging missions. The priority of some stars may be reduced if the imaging search space in planet mass and semimajor axis has already been ruled out by Doppler observations. Conversely, other stars may be more attractive if Doppler surveys can rule out dynamically disruptive giant planets and the imaging missions are sensitive to smaller planets that are undetectable by the Doppler measurements.

This paper is the product of a study titled 'Radial Velocity Data Review in Support of Direct Imaging Mission Concept Reports' that was carried out by the authors under contract from NASA Jet Propulsion Laboratory. We were charged with identifying the 
nearby stars that are likely targets of Exo-C, Exo-S, and WFIRST-AFTA and that have historical Doppler measurements at Lick Observatory and Keck Observatory taken by the California Planet Survey (Howard et al. 2010a). For stars with Doppler measurements, we were to estimate the region of discovery space (planet masses and orbital semi-major axes) in which planets can be ruled out. These "completeness" estimates, and the lists of discovered planets orbiting those stars, provide a valuable input to the planning and eventual operation of space-based planet imaging missions.

This report is organized as follows. The next two sections describe target lists for the Exo-S, Exo-C, and WFIRST-AFTA imaging studies (Sec. 2) and the California Planet Search using Doppler spectroscopy (Sec. 3). We review Keplerian orbits in Sec. 4, describe our automated Doppler planet search in Sec.5, and simulate the sensitivity of future Doppler searches in Sec. 6. Machine-readable data files included with this report are described in Appendix A and can be downloaded from http://exoplanetarchive.ipac.caltech.edu/docs/contributed_data.html. The remaining appendices provide exhaustive data that is too large for the main text. Appendix $\mathrm{B}$ includes a complete list of the Exo-C, Exo-S, and WFIRST-AFTA stars that are not part of our Lick/Keck Doppler survey and the likely reasons for their exclusion. Appendix C contains plots summarizing the search results and completeness estimates for every star with Doppler measurements.

\section{Target Lists for the Exo-C, Exo-S, and WFIRST-AFTA Studies}

The Exo-S, Exo-C, and WFIRST-AFTA study teams selected preliminary lists of nearby stars for their missions. These target lists contain real stars and allow for simulations of mission performance that depend on genuine stellar properties. Target lists were provided to us by representatives of the study teams (M. Turnbull and K. Stapelfeldt) and are current as of July 31, 2014. Stellar properties were taken from the "Properties of Nearby Stars" page on the NASA ExEP websit ${ }^{1}$. This catalog was assembled by M. Turnbull and includes catalog numbers from Hipparcos, Henry Draper (HD), and Gliese catalogs, as well as sky coordinates, distances, $V$-band brightnesses, $B-V$ colors, spectral types, and luminosities. These Exo-S, Exo-C, and WFIRST-AFTA target stars are listed in two tables below. Table 1 includes stars for which we have Doppler measurements and provide completeness measurements. Stellar masses (to convert orbital periods into semi-major axes) are mostly derived from stellar synthesis fits using Spectroscopy Made Easy (Valenti \& Fischer 2005). Table 2 gives a summary of the reasons that stars were not part of the Lick/Keck doppler planet search

\footnotetext{
${ }^{1}$ See http://nexsci.caltech.edu/missions/EXEP/EXEPstarlist.html
} 
programs. Table B.1 lists Exo-S, Exo-C, and WFIRST-AFTA targets without Doppler measurements. We adopt HD numbers as the primary stellar names throughout this report because they are the standard star names used internally for our Keck program. 
Table 1. Imaging Target Stars with Doppler Measurements

\begin{tabular}{|c|c|c|c|c|c|c|c|c|c|c|}
\hline $\begin{array}{l}\text { Hipp. } \\
\text { No. }\end{array}$ & $\begin{array}{l}\text { HD } \\
\text { No. }\end{array}$ & $\begin{array}{l}\text { Gliese } \\
\text { No. }\end{array}$ & $\begin{array}{c}\text { Target }^{\mathrm{a}} \\
\text { Lists }\end{array}$ & $\begin{array}{l}\text { Dist. } \\
\text { (pc) }\end{array}$ & $\begin{array}{c}V \\
(\mathrm{mag})\end{array}$ & $\begin{array}{c}B-V \\
(\mathrm{mag})\end{array}$ & $\begin{array}{l}T_{\text {eff }} \\
(\mathrm{K})\end{array}$ & $\begin{array}{c}M_{\star} \\
\left(M_{\odot}\right)\end{array}$ & Sp. T. & Notes \\
\hline 544 & 166 & 5 & S & 13.671 & 6.060 & 0.752 & 5577 & 0.987 & $\mathrm{~K} 0 \mathrm{~V}$ & \\
\hline 3093 & 3651 & 27 & $\mathrm{~S}$ & 11.060 & 5.880 & 0.850 & 5221 & 0.920 & $\mathrm{~K} 0 \mathrm{~V}$ & \\
\hline 3821 & 4614 & $34 \mathrm{~A}$ & $\mathrm{~S}, \mathrm{C}, \mathrm{A}$ & 5.944 & 3.452 & 0.569 & 5941 & 0.986 & G3V & \\
\hline 3765 & 4628 & 33 & $\mathrm{~S}, \mathrm{C}$ & 7.455 & 5.740 & 0.890 & 4944 & 0.728 & $\mathrm{~K} 1 \mathrm{~V}$ & \\
\hline 7513 & 9826 & 61 & $\mathrm{C}, \mathrm{A}$ & 13.492 & 4.090 & 0.536 & 6213 & 1.310 & $\mathrm{~F} 8 \mathrm{~V}$ & \\
\hline 7981 & 10476 & 68 & $\mathrm{~S}, \mathrm{C}, \mathrm{A}$ & 7.532 & 5.240 & 0.836 & 5181 & 0.863 & $\mathrm{~K} 1 \mathrm{~V}$ & \\
\hline 8102 & 10700 & 71 & $\mathrm{~S}, \mathrm{C}, \mathrm{A}$ & 3.650 & 3.490 & 0.727 & 5283 & 0.762 & G8V & \\
\hline 8362 & 10780 & 75 & $\mathrm{~S}$ & 10.067 & 5.630 & 0.804 & 5327 & 0.879 & $\mathrm{~K} 0 \mathrm{~V}$ & \\
\hline 12114 & 16160 & $105 \mathrm{~A}$ & $\mathrm{~S}$ & 7.180 & 5.819 & 0.984 & 4866 & 0.750 & $\mathrm{~K} 3 \mathrm{~V}$ & \\
\hline 13402 & 17925 & 117 & S & 10.352 & 6.046 & 0.862 & 5236 & 0.800 & $\mathrm{~K} 1 \mathrm{~V}$ & \\
\hline 14632 & 19373 & 124 & $\mathrm{~S}, \mathrm{C}, \mathrm{A}$ & 10.541 & 4.050 & 0.595 & 6032 & 1.204 & G0V & \\
\hline 14954 & 19994 & $128 \mathrm{~A}$ & A & 22.580 & 5.070 & 0.580 & 6188 & 1.300 & $\mathrm{~F} 8.5 \mathrm{~V}$ & \\
\hline 15457 & 20630 & 137 & $\mathrm{~S}, \mathrm{C}, \mathrm{A}$ & 9.140 & 4.842 & 0.681 & 5742 & 0.900 & G5V & \\
\hline 16537 & 22049 & 144 & $\mathrm{C}, \mathrm{A}$ & 3.213 & 3.714 & 0.881 & 5146 & 0.815 & $\mathrm{~K} 2 \mathrm{~V}$ & young \\
\hline 16852 & 22484 & 147 & $\mathrm{~S}, \mathrm{C}, \mathrm{A}$ & 13.963 & 4.290 & 0.575 & 6038 & 1.206 & $\mathrm{~F} 8 \mathrm{~V}$ & \\
\hline 17378 & 23249 & 150 & $\mathrm{~S}, \mathrm{C}$ & 9.041 & 3.518 & 0.934 & 5095 & 1.163 & K0IV & subgiant \\
\hline 18859 & 25457 & 159 & $\mathrm{~A}$ & 18.830 & 5.380 & 0.520 & 6308 & 1.179 & F7V & young \\
\hline 19849 & 26965 & $166 \mathrm{~A}$ & $\mathrm{~S}, \mathrm{C}, \mathrm{A}$ & 4.984 & 4.430 & 0.820 & 5151 & 0.775 & $\mathrm{~K} 1 \mathrm{~V}$ & \\
\hline 22263 & 30495 & 177 & $\mathrm{~S}$ & 13.277 & 5.486 & 0.632 & 5759 & 1.000 & G3V & \\
\hline 22449 & 30652 & 178 & $\mathrm{~S}, \mathrm{C}, \mathrm{A}$ & 8.068 & 3.167 & 0.464 & 6424 & 1.236 & $\mathrm{~F} 6 \mathrm{~V}$ & \\
\hline 23311 & 32147 & 183 & $\mathrm{~S}$ & 8.708 & 6.225 & 1.049 & 4827 & 0.821 & $\mathrm{~K} 3 \mathrm{~V}$ & \\
\hline 23835 & 32923 & $188 \mathrm{~A}$ & $\mathrm{~S}, \mathrm{C}, \mathrm{A}$ & 15.434 & 4.910 & 0.657 & 5694 & 1.001 & G4V & \\
\hline 24813 & 34411 & 197 & $\mathrm{~S}, \mathrm{C}, \mathrm{A}$ & 12.631 & 4.691 & 0.614 & 5911 & 1.105 & G0V & \\
\hline 26779 & 37394 & 211 & $\mathrm{~S}$ & 12.277 & 6.198 & 0.840 & 5351 & 0.919 & $\mathrm{~K} 1 \mathrm{~V}$ & young \\
\hline 29650 & 43042 & 3390 & $\mathrm{~A}$ & 20.810 & 5.200 & 0.430 & 6418 & 1.000 & F5.5IV-V & early type \\
\hline 32480 & 48682 & 245 & $\mathrm{~A}$ & 16.720 & 5.240 & 0.550 & 6064 & 1.177 & G0V & \\
\hline 39780 & 67228 & $\ldots$ & $\mathrm{A}$ & 23.290 & 5.300 & 0.640 & 5862 & 1.220 & G2IV & \\
\hline 40693 & 69830 & 302 & S & 12.494 & 5.943 & 0.754 & 5361 & 0.871 & $\mathrm{~K} 0 \mathrm{~V}$ & \\
\hline 40843 & 69897 & 303 & A & 18.270 & 5.130 & 0.470 & 6294 & 1.095 & $\mathrm{~F} 6 \mathrm{~V}$ & early type \\
\hline 42438 & 72905 & 311 & $\mathrm{~S}$ & 14.355 & 5.630 & 0.618 & 5920 & 1.000 & $\mathrm{G} 1.5 \mathrm{Vb}$ & young \\
\hline 43587 & 75732 & $324 \mathrm{~A}$ & $\mathrm{~S}$ & 12.341 & 5.960 & 0.869 & 5235 & 0.966 & G8V & \\
\hline 47592 & 84117 & 364 & $\mathrm{~S}, \mathrm{C}, \mathrm{A}$ & 15.013 & 4.924 & 0.534 & 6152 & 1.159 & G0V & \\
\hline 48113 & 84737 & 368 & $\mathrm{~A}$ & 18.370 & 5.080 & 0.620 & 5960 & 1.164 & G0V & \\
\hline 49081 & 86728 & 376 & $\mathrm{~S}, \mathrm{~A}$ & 15.047 & 5.375 & 0.671 & 5700 & 1.095 & G1V & \\
\hline 51459 & 90839 & 395 & $\mathrm{~S}, \mathrm{C}, \mathrm{A}$ & 12.780 & 4.820 & 0.541 & 6126 & 1.200 & F8V & \\
\hline 53721 & 95128 & 407 & A & 14.060 & 5.030 & 0.610 & 5882 & 1.083 & G1V & \\
\hline
\end{tabular}




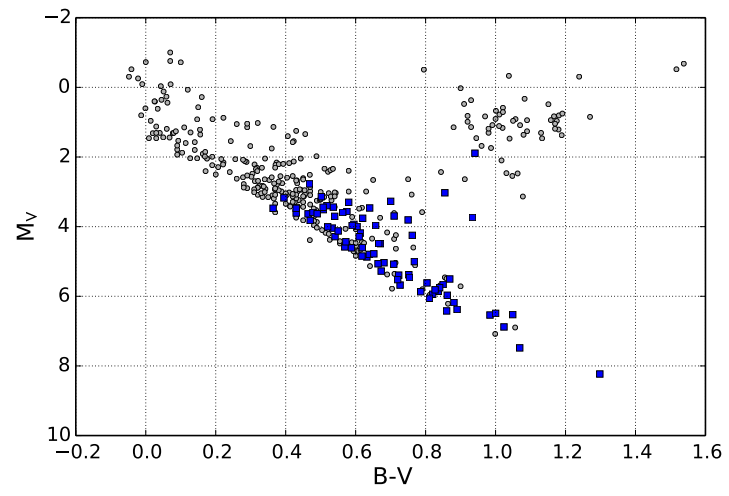

Fig. 1.- HR diagram of stars in the Exo-S, Exo-C, and WFIRST-AFTA target lists with (blue squares) and without (gray circles) Doppler observations from Lick and Keck Observatories. Doppler searches favor late-F through mid-M type dwarfs for planet detectability. Imaging searches for planets in reflected light typically prefer bright stars, which are dominated by early type stars. The region of overlap encompasses primarily F8-K0 dwarfs. 
Table 1-Continued

\begin{tabular}{|c|c|c|c|c|c|c|c|c|c|c|}
\hline $\begin{array}{l}\text { Hipp. } \\
\text { No. }\end{array}$ & $\begin{array}{l}\text { HD } \\
\text { No. }\end{array}$ & $\begin{array}{l}\text { Gliese } \\
\text { No. }\end{array}$ & $\begin{array}{c}\text { Target }^{\mathrm{a}} \\
\text { Lists }\end{array}$ & $\begin{array}{l}\text { Dist. } \\
\text { (pc) }\end{array}$ & $\begin{array}{c}V \\
(\mathrm{mag})\end{array}$ & $\begin{array}{c}B-V \\
(\mathrm{mag})\end{array}$ & $\begin{array}{l}T_{\text {eff }} \\
(\mathrm{K})\end{array}$ & $\begin{array}{c}M_{\star} \\
\left(M_{\odot}\right)\end{array}$ & Sp. T. & Notes \\
\hline 56452 & 100623 & $432 \mathrm{~A}$ & S & 9.559 & 5.959 & 0.811 & 5189 & 0.747 & $\mathrm{~K} 0 \mathrm{~V}$ & \\
\hline 56997 & 101501 & 434 & $\mathrm{~S}, \mathrm{C}, \mathrm{A}$ & 9.612 & 5.307 & 0.723 & 5488 & 0.913 & G8V & \\
\hline 57443 & 102365 & $442 \mathrm{~A}$ & $\mathrm{~S}, \mathrm{C}, \mathrm{A}$ & 9.220 & 4.890 & 0.664 & 5630 & 0.863 & G5V & \\
\hline 57757 & 102870 & 449 & $\mathrm{~S}, \mathrm{C}, \mathrm{A}$ & 10.929 & 3.590 & 0.518 & 6161 & 1.100 & F9V & \\
\hline 58576 & 104304 & 454 & $\mathrm{~S}$ & 12.763 & 5.533 & 0.768 & 5565 & 1.018 & G8V & \\
\hline 61317 & 109358 & 475 & $\mathrm{~S}, \mathrm{C}, \mathrm{A}$ & 8.440 & 4.241 & 0.588 & 5930 & 0.929 & G0V & \\
\hline 64394 & 114710 & 502 & $\mathrm{~S}, \mathrm{C}, \mathrm{A}$ & 9.129 & 4.237 & 0.572 & 6075 & 1.150 & G0V & \\
\hline 64408 & 114613 & 501.2 & $\mathrm{~A}$ & 20.670 & 4.850 & 0.700 & 5782 & 1.266 & G3V & \\
\hline 64792 & 115383 & 504 & $\mathrm{~A}$ & 17.560 & 5.190 & 0.590 & 6234 & 2.310 & G0IV & young \\
\hline 64924 & 115617 & 506 & $\mathrm{~S}, \mathrm{C}, \mathrm{A}$ & 8.555 & 4.740 & 0.709 & 5571 & 0.954 & G5V & \\
\hline 65721 & 117176 & 512.1 & $\mathrm{~A}$ & 17.990 & 4.970 & 0.710 & 5545 & 1.109 & G5V & \\
\hline 67275 & 120136 & $527 \mathrm{~A}$ & $\mathrm{~S}, \mathrm{C}, \mathrm{A}$ & 15.618 & 4.479 & 0.508 & 6387 & 1.341 & F7V & \\
\hline 71284 & 128167 & 557 & $\mathrm{~S}, \mathrm{C}, \mathrm{A}$ & 15.833 & 4.470 & 0.364 & 6566 & 1.500 & F3V & early type \\
\hline 72659 & 131156 & $566 \mathrm{~A}$ & $\mathrm{~S}, \mathrm{C}, \mathrm{A}$ & 6.776 & 4.675 & 0.720 & 5380 & 0.920 & G7V & young \\
\hline 73184 & 131977 & $570 \mathrm{~A}$ & $\mathrm{~S}, \mathrm{C}$ & 5.861 & 5.720 & 1.024 & 4744 & 0.760 & $\mathrm{~K} 4 \mathrm{~V}$ & \\
\hline 73996 & 134083 & 578 & $\mathrm{~A}$ & 19.550 & 4.930 & 0.430 & 6435 & 1.210 & $\mathrm{~F} 5 \mathrm{~V}$ & early type \\
\hline 75181 & 136352 & 582 & $\mathrm{~S}$ & 14.810 & 5.650 & 0.639 & 5672 & 0.848 & G4V & \\
\hline 77257 & 141004 & 598 & $\mathrm{~S}, \mathrm{C}, \mathrm{A}$ & 12.124 & 4.413 & 0.604 & 5936 & 1.036 & G0Vvar & \\
\hline 77760 & 142373 & 602 & $\mathrm{~S}, \mathrm{C}, \mathrm{A}$ & 15.893 & 4.599 & 0.563 & 5861 & 1.100 & F9V & \\
\hline 78072 & 142860 & 603 & $\mathrm{~S}, \mathrm{C}, \mathrm{A}$ & 11.254 & 3.850 & 0.478 & 6262 & 1.300 & $\mathrm{~F} 6 \mathrm{~V}$ & \\
\hline 79672 & 146233 & 616 & $\mathrm{~S}$ & 13.900 & 5.496 & 0.652 & 5791 & 1.038 & G5V & \\
\hline 81300 & 149661 & 631 & S & 9.751 & 5.760 & 0.827 & 5277 & 0.883 & $\mathrm{~K} 1 \mathrm{~V}$ & young \\
\hline 84862 & 157214 & 672 & $\mathrm{~S}, \mathrm{~A}$ & 14.327 & 5.383 & 0.619 & 5697 & 0.871 & G0V & \\
\hline 86974 & 161797 & $695 \mathrm{~A}$ & $\mathrm{~S}, \mathrm{C}, \mathrm{A}$ & 8.310 & 3.405 & 0.750 & 5641 & 1.142 & G5IV & subgiant \\
\hline 89962 & 168723 & 711 & $\mathrm{C}$ & 18.543 & 3.232 & 0.941 & 4975 & 1.721 & K0III-IV & subgiant \\
\hline 91438 & 172051 & 722 & S & 13.084 & 5.860 & 0.673 & 5564 & 0.855 & G5V & \\
\hline 92043 & 173667 & 725.2 & $\mathrm{C}, \mathrm{A}$ & 19.209 & 4.189 & 0.468 & 6423 & 1.100 & F6V & \\
\hline 95447 & 182572 & 759 & $\mathrm{~S}, \mathrm{~A}$ & 15.177 & 5.157 & 0.762 & 5656 & 1.141 & G8IVvar & \\
\hline 96100 & 185144 & 764 & $\mathrm{~S}, \mathrm{C}, \mathrm{A}$ & 5.754 & 4.668 & 0.786 & 5246 & 0.801 & K0V & \\
\hline 96441 & 185395 & $765 \mathrm{~A}$ & $\mathrm{C}, \mathrm{A}$ & 18.335 & 4.490 & 0.395 & 6594 & 1.380 & F4V & early type \\
\hline 97675 & 187691 & $768.1 \mathrm{~A}$ & $\mathrm{~A}$ & 19.190 & 5.120 & 0.540 & 6139 & 1.370 & F8V & \\
\hline 98036 & 188512 & $771 \mathrm{~A}$ & $\mathrm{~S}, \mathrm{C}$ & 13.699 & 3.711 & 0.855 & 5163 & 1.257 & G8IVvar & \\
\hline 99461 & 191408 & $783 \mathrm{~A}$ & $\mathrm{~S}, \mathrm{C}, \mathrm{A}$ & 6.015 & 5.317 & 0.860 & 4922 & 0.686 & $\mathrm{~K} 2.5 \mathrm{~V}$ & \\
\hline 104214 & 201091 & $820 \mathrm{~A}$ & $\mathrm{~S}, \mathrm{C}, \mathrm{A}$ & 3.496 & 5.200 & 1.069 & 4655 & 0.662 & $\mathrm{~K} 5 \mathrm{~V}$ & \\
\hline 104217 & 201092 & $820 \mathrm{~B}$ & $\mathrm{~S}$ & 3.496 & 5.950 & 1.298 & 4145 & 0.548 & $\mathrm{~K} 7 \mathrm{~V}$ & \\
\hline 109422 & 210302 & 849.1 & $\mathrm{~A}$ & 18.280 & 4.940 & 0.490 & 6339 & 1.299 & F6V & early type \\
\hline
\end{tabular}


Figure1 1 shows the Exo-S, Exo-C, and WFIRST-AFTA targets in a Hertzsprung-Russell diagram. Stars with Doppler measurements from Lick or Keck Observatories are highlighted in blue. These stars are nearly all main sequence stars with spectral type F8 and later $(B-V>0.4)$ with a handful of K-type subgiants and giants. Table 2 summarizes the number of stars in each of the three imaging missions for which we have Lick and/or Keck Doppler measurements. The table also attempts to reconstruct the reasons that stars in the imaging mission target lists were not observed by the Lick/Keck searches.

\section{Doppler Targets and Measurements}

The historic Lick Planet Search (Fischer et al. 2014) with the the Hamilton spectrograph (Vogt 1987) began in 1987 with a spectrum of $\tau$ Ceti. The original target list included 120 stars from the Bright Star Catalog (Hoffleit \& Jaschek 1982) and the Gliese-Jahreiss catalog (Gliese 1969; Gliese \& Jahreiß 1979). In 1997 many fainter stars were moved to the new Keck Planet Search and 200 additional stars were added to the Lick Planet Search. An additional 67 metal-rich stars were added in 2001, bringing the total in the Lick Planet Search to 367 stars (Fischer et al. 2014). The Lick target list was dominated by hotter and brighter stars (typically $B-V=0.4-0.7$ ). Earlier type stars were generally excluded because of increased Doppler noise $\left(>5 \mathrm{~ms}^{-1}\right)$. Observations continued through 2011.

Doppler velocities were measured for the Lick and Keck Planet Searches with the aid of an iodine cell. These glass cells containing gaseous $\mathrm{I}_{2}$ act a transmission filter, imprinting thousands of narrow iodine absorption lines on the stellar spectra in the wavelength region 5000-6200 A. The dense set of molecular absorption lines provide a robust wavelength fiducial against which Doppler shifts are measured, and place strong constraints on the shape of

Table 1 - Continued

\begin{tabular}{ccrcccccccc}
\hline \hline $\begin{array}{c}\text { Hipp. } \\
\text { No. }\end{array}$ & $\begin{array}{c}\text { HD } \\
\text { No. }\end{array}$ & $\begin{array}{c}\text { Gliese } \\
\text { No. }\end{array}$ & $\begin{array}{c}\text { Target } \\
\text { Lists }\end{array}$ & $\begin{array}{c}\text { Dist. } \\
(\mathrm{pc})\end{array}$ & $\begin{array}{c}V \\
(\mathrm{mag})\end{array}$ & $\begin{array}{c}B-V \\
(\mathrm{mag})\end{array}$ & $\begin{array}{c}T_{\text {eff }} \\
(\mathrm{K})\end{array}$ & $\begin{array}{c}M_{\star} \\
\left(M_{\odot}\right)\end{array}$ & Sp. T. & Notes \\
\hline 112447 & 215648 & $872 \mathrm{~A}$ & S, C, A & 16.297 & 4.200 & 0.502 & 6204 & 1.300 & F7V & \\
113357 & 217014 & 882 & S & 15.608 & 5.452 & 0.666 & 5787 & 1.064 & G5V & \\
114622 & 219134 & 892 & S, C & 6.543 & 5.570 & 1.000 & 4835 & 0.782 & K3V & \\
116771 & 222368 & 904 & S, C, A & 13.714 & 4.130 & 0.507 & 6204 & 1.170 & F7V & \\
\hline
\end{tabular}

a Target list code: $\mathrm{S}=$ starshade study mission target, $\mathrm{C}=$ coronagraph study mission target, $\mathrm{A}=$ WFIRST-AFTA study mission target. 
Table 2. Summary of Reasons for Excluding Stars from Lick/Keck Programs ${ }^{a}$

\begin{tabular}{|c|c|c|c|c|c|c|}
\hline \multirow[t]{2}{*}{ Mission } & \multirow[t]{2}{*}{ Total Stars } & \multirow[t]{2}{*}{ Have RVs ${ }^{b}$} & \multicolumn{4}{|c|}{ No RVs } \\
\hline & & & $\mathrm{Hot}^{\mathrm{c}}$ & Southern $^{\mathrm{d}}$ & Evolved $^{\mathrm{e}}$ & Binary $^{f}$ \\
\hline Exo-S (S) & 127 & 57 & 19 & 24 & 3 & 22 \\
\hline Exo-C (C) & 249 & 40 & 112 & 43 & 39 & 33 \\
\hline WFIRST-AFTA (A) & 263 & 51 & 125 & 51 & 4 & 38 \\
\hline Total $(\mathrm{S}+\mathrm{C}+\mathrm{A})$ & 376 & 76 & 148 & 71 & 40 & 51 \\
\hline
\end{tabular}

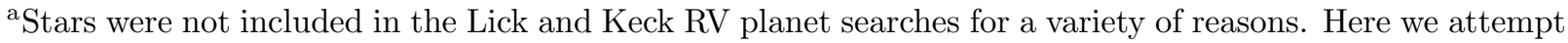
to reconstruct the reasons that stars were not added to those programs, focussing on four non-exclusive and incomplete categories.

${ }^{\mathrm{b}}$ All stars with RVs are listed in Table 1 .

${ }^{\mathrm{c}}$ The number of stars with $B-V<0.44$, corresponding to spectral type F5 V.

${ }^{\mathrm{d}}$ The number of stars with declination $<-30^{\circ}$.

${ }^{\mathrm{e}}$ The number of stars not listed as dwarf spectral types in Simbad.

${ }^{\mathrm{f}} \mathrm{A}$ rough estimate of the number of stellar multiples. We include targets noted as 'spectroscopic binary' or with multiple, distinct spectral types listed in Simbad, or a listing in the Washington Double Star Catalog. 
the spectrometer instrumental profile at the time of each observation (Marcy \& Butler 1992; Valenti et al. 1995). Relative radial velocities were measured using a forward-modeling technique that simultaneously solves for the spatially-varying instrumental profile, wavelength solution, and Doppler shift of each spectrum (Butler et al. 1996).

The Lick Planet Search was world-leading in the discovery and characterization of exoplanets. Major discoveries include the confirmation of 51 Peg b (Marcy et al. 1997), the discovery of the first planet in an eccentric orbit (Marcy \& Butler 1996), the first multiplanet system (Butler et al. 1999), the first sub-Saturn-mass planets (Marcy et al. 2000), the discovery of 70 of the first 100 extrasolar planets (Marcy \& Butler 2000), and the first planet search completeness limits for a large sample of stars (Cumming et al. 1999).

The Keck Planet Search has been in operation since 1996 July using the HIRES echelle spectrometer on the Keck I telescope (Vogt et al. 1994). Selection of the target stars is described in Wright et al. (2004) and Marcy et al. (2005b). By 2004, the Keck target list included $\sim 1000$ stars with spectral types F5-M5. These stars generally lie close to the main sequence and are chromospherically quiet. Most have $B-V>0.55$, declination $>-35^{\circ}$, and have no stellar companion within $2^{\prime \prime}$ that would introduce a second, complicating spectrum into the Doppler analysis. Young, magnetically active stars are excluded from the primary planet search because of increased Doppler noise. The Keck Planet Search was expanded over the years with bright stars added from the original Lick program as it winded down, as well as large samples of subgiants (Johnson et al. 2007), metal-rich main sequence stars (Fischer et al. 2005; Robinson et al. 2007), young stars (Hillenbrand et al. 2014), and other stellar populations.

The Keck Planet Search was also highly successful at discovering and characterizing exoplanets. Highlights include discoveries of the the first Neptune-size planets (Maness et al. 2007) and numerous super-Earths (Howard et al. 2009, 2011a. b, 2014), demonstration of the planet-metallicity correlation (Fischer et al. 2005), statistical studies of planet occurrence (Marcy et al. 2005a; Cumming et al. 2008; Howard et al. 2010b), and recently measurements of transiting planets discovered by NASA's Kepler Mission (Marcy et al. 2014).

Combining RVs and high-contrast imaging to discover planets, brown dwarfs, and other orbiting companions has a strong heritage at Keck Observatory. The TRENDS program has used the long RV time series to detect accelerations from wide companions. Followup imaging with Keck-NIRC2 and other instruments has revealed several brown dwarfs and more massive companions (Crepp et al. 2012a b, 2013a, b, 2014). Similarly, statistical analyses of the long-period accelerations in the Keck RV time series have been used to estimate the fraction of stars with wide-orbit planets (Knutson et al. 2014; Montet et al. 2014) 
For this Doppler planet search completeness study, we analyzed RVs from four instruments with independent RV zero points at Lick and Keck. Instrument codes $\mathrm{p}$ and 1 refer to data from the Hamilton spectrograph on the Shane Telescope at Lick Observatory before and after a major detector upgrade (Fischer et al. 2014). Similarly, codes k and j refer to data taken with HIRES on the Keck I telescope before and after an upgrade in 2004 (Vogt et al. 1994). The detector and/or optics in the Hamilton spectrograph were upgraded several times during its 25 years of operation. The commissioning of the Lick Planet Search in 1987 used a TI $800 \times 800$ pixel CCD that was upgraded to a $2048 \times 2048$ pixel detector in 1990 (dewar 16). During this era, nightly velocity corrections were made at the level of $10 \mathrm{~m}$ $\mathrm{s}^{-1}$ to account for uncontrolled changes in the instrumental setup (Fischer et al. 2014). In November 1994 the Schmidt camera optics were replaced and a field flattener and new CCD were installed. We treat data prior to November of 1994 as a single instrument with instrument code "p". After the 1994 camera and optics upgrade the CCD was changed another two times in February of 1998, and 2001. We treat these CCDs as a single instrument in our analysis with telescope code "l". RV zero-point offsets between the three dewars on the telescope from 1998 to 2011 have been solved for and removed as best as possible by (Fischer et al. 2014). They found negligible average offsets $\left(<0.5 \mathrm{~m} \mathrm{~s}^{-1}\right)$ between all cameras except that RVs collected using the final CCD (dewar 8) were on average $13.1 \mathrm{~m} \mathrm{~s}^{-1}$ higher than the rest. Fischer et al. (2014) do not mention the variance on the mean offset measured for each instrument so we can not say how robustly these offsets have been removed for any given star. Residual uncorrected offsets can lead to spurious planet candidate detections in our automated planet detection pipeline (see Figure C.7) and these cases are all mentioned explicitly in Section 5.2. Dewar 8 was in operation until 2011 when the iodine cell failed and the Lick Planet Search ceased. Lick RVs were taken from Fischer et al. (2014) with no additional processing (with the exception of binning measurements in 2 hour intervals, as needed). The CCD on HIRES was upgraded and a new field flattener was installed on 19 August 2004, introducing an RV zero-point uncertainty. Data taken on or before the HIRES upgrade are assigned the instrument code "k", and post-upgrade data use the code "j". Keck RVs reported here run through 26 August 2014.

Table 3 summarizes the observational histories of each star, including the time baseline, UT date of the first RV measurement, and the number and scatter (RMS) of the RVs for each instrument code. When multiple measurements were gathered in short succession for the same star, we binned the data in $2 \mathrm{hr}$ intervals. 
Table 3. Properties of Doppler Measurements

\begin{tabular}{|c|c|c|c|c|c|c|c|c|c|c|c|}
\hline \multirow{3}{*}{$\begin{array}{c}\text { Hipp. } \\
\text { no. }\end{array}$} & \multirow{3}{*}{$\begin{array}{l}\text { HD } \\
\text { no. }\end{array}$} & \multirow{3}{*}{$\begin{array}{c}t_{\text {span }} \\
(\mathrm{yr})\end{array}$} & \multirow{3}{*}{ Start Date } & \multicolumn{4}{|c|}{ Number of RVs } & \multicolumn{4}{|c|}{$\operatorname{RMS}$ of RVs $\left(\mathrm{ms}^{-1}\right)^{\mathrm{a}}$} \\
\hline & & & & \multicolumn{2}{|c|}{ Lick Dewars } & \multicolumn{2}{|c|}{ Keck Dewars } & \multicolumn{2}{|c|}{ Lick Dewars } & \multicolumn{2}{|c|}{ Keck Dewars } \\
\hline & & & & $\mathrm{p}$ & 1 & $\mathrm{k}$ & $\mathrm{j}$ & $\mathrm{p}$ & 1 & $\mathrm{k}$ & $\mathrm{j}$ \\
\hline 544 & 166 & 27.1 & 1987-06-13 & 22 & 22 & $\cdots$ & 17 & 18.8 & 20.7 & $\ldots$ & 17.5 \\
\hline 3093 & 3651 & 26.3 & 1987-09-09 & 16 & 139 & 29 & 54 & 11.1 & 7.2 & 3.7 & 3.3 \\
\hline 3821 & 4614 & 18.9 & 1995-09-09 & $\cdots$ & 21 & $\cdots$ & 48 & $\cdots$ & 10.4 & $\cdots$ & 10.2 \\
\hline 3765 & 4628 & 26.9 & 1987-09-08 & 15 & 37 & $\cdots$ & 89 & 10.4 & 6.1 & $\ldots$ & 2.7 \\
\hline 7513 & 9826 & 26.9 & 1987-09-08 & 17 & 312 & $\cdots$ & 8 & 30.7 & 13.0 & $\cdots$ & 2.9 \\
\hline 7981 & 10476 & 26.8 & 1987-09-09 & 15 & 49 & 65 & 69 & 8.9 & 8.1 & 5.1 & 2.7 \\
\hline 8102 & 10700 & 27.0 & 1987-09-08 & 95 & 535 & 87 & 191 & 8.9 & 6.0 & 3.9 & 2.5 \\
\hline 8362 & 10780 & 15.6 & $1998-12-05$ & $\cdots$ & 16 & $\ldots$ & 16 & $\ldots$ & 6.2 & $\ldots$ & 4.1 \\
\hline 12114 & 16160 & 25.9 & 1987-09-10 & 14 & 48 & $\ldots$ & 61 & 12.4 & 5.8 & $\ldots$ & 2.5 \\
\hline 13402 & 17925 & 10.9 & $1995-02-20$ & $\ldots$ & 15 & $\ldots$ & $\cdots$ & $\ldots$ & 32.1 & $\ldots$ & $\cdots$ \\
\hline 14632 & 19373 & 27.0 & 1987-09-08 & 71 & 158 & 8 & 74 & 9.0 & 8.8 & 4.7 & 2.9 \\
\hline 15457 & 20630 & 24.1 & 1987-09-08 & 25 & 19 & $\ldots$ & $\ldots$ & 21.9 & 25.6 & $\ldots$ & $\ldots$ \\
\hline 16537 & 22049 & 27.0 & 1987-09-08 & 49 & 127 & $\ldots$ & 61 & 17.8 & 9.6 & $\ldots$ & 8.3 \\
\hline 16852 & 22484 & 18.8 & $1995-02-18$ & $\cdots$ & 34 & $\ldots$ & 5 & $\ldots$ & 10.0 & $\ldots$ & 5.3 \\
\hline 17378 & 23249 & 16.8 & 1997-01-14 & $\ldots$ & $\ldots$ & 20 & 20 & $\ldots$ & $\ldots$ & 4.1 & 3.5 \\
\hline 19849 & 26965 & 19.5 & $1995-02-21$ & $\ldots$ & 77 & 7 & 92 & $\ldots$ & 7.8 & 2.0 & 3.3 \\
\hline 22263 & 30495 & 11.0 & 2000-10-19 & $\ldots$ & 42 & $\ldots$ & .. & $\ldots$ & 16.4 & $\cdots$ & $\ldots$ \\
\hline 22449 & 30652 & 11.1 & 2000-09-09 & $\ldots$ & 48 & $\ldots$ & $\ldots$ & $\ldots$ & 31.0 & $\ldots$ & $\ldots$ \\
\hline 23311 & 32147 & 26.3 & $1987-09-08$ & 7 & 23 & $\cdots$ & 96 & 7.8 & 3.6 & $\ldots$ & 2.8 \\
\hline 23835 & 32923 & 14.7 & $1998-12-05$ & $\ldots$ & 24 & $\ldots$ & 55 & $\ldots$ & 8.5 & $\ldots$ & 5.7 \\
\hline 24813 & 34411 & 26.0 & 1987-09-10 & 17 & 259 & 8 & 60 & 8.6 & 6.7 & 8.6 & 2.9 \\
\hline 26779 & 37394 & 16.8 & $1995-11-11$ & $\ldots$ & 8 & $\ldots$ & 12 & $\ldots$ & 10.7 & $\ldots$ & 15.4 \\
\hline 40693 & 69830 & 13.3 & 2000-11-12 & $\ldots$ & 31 & $\ldots$ & 106 & $\ldots$ & 7.4 & $\ldots$ & 3.2 \\
\hline 42438 & 72905 & 11.0 & $2002-10-25$ & $\ldots$ & $\ldots$ & 4 & 12 & $\ldots$ & $\ldots$ & 45.0 & 40.9 \\
\hline 43587 & 75732 & 25.3 & 1989-02-21 & 14 & 274 & 24 & 193 & 19.7 & 6.8 & 4.4 & 3.0 \\
\hline 47592 & 84117 & 9.2 & 2004-11-29 & $\cdots$ & $\ldots$ & $\ldots$ & 44 & $\cdots$ & $\cdots$ & $\cdots$ & 4.1 \\
\hline 49081 & 86728 & 26.5 & $1987-06-13$ & 15 & 59 & $\ldots$ & 44 & 9.2 & 8.0 & $\ldots$ & 3.2 \\
\hline 51459 & 90839 & 17.0 & $1987-12-20$ & 16 & 12 & $\ldots$ & $\cdots$ & 18.5 & 9.3 & $\ldots$ & $\cdots$ \\
\hline 56452 & 100623 & 17.1 & $1996-12-01$ & $\ldots$ & $\ldots$ & 16 & 3 & $\ldots$ & $\ldots$ & 19.0 & 6.1 \\
\hline 56997 & 101501 & 24.8 & $1987-06-12$ & 20 & 69 & $\ldots$ & 4 & 17.8 & 10.6 & $\ldots$ & 15.9 \\
\hline 57443 & 102365 & 6.6 & 2007-05-26 & $\ldots$ & $\ldots$ & $\ldots$ & 16 & $\ldots$ & $\ldots$ & $\ldots$ & 2.5 \\
\hline 57757 & 102870 & 21.4 & 1987-06-13 & 28 & 70 & $\ldots$ & .. & 20.2 & 12.6 & $\ldots$ & $\ldots$ \\
\hline 58576 & 104304 & 13.2 & 2001-05-11 & $\cdots$ & 23 & $\cdots$ & 36 & $\ldots$ & 8.6 & $\ldots$ & 2.5 \\
\hline 61317 & 109358 & 14.4 & 2000-02-09 & $\cdots$ & $\ldots$ & 10 & 69 & $\ldots$ & $\cdots$ & 3.9 & 3.2 \\
\hline
\end{tabular}


Table 3-Continued

\begin{tabular}{|c|c|c|c|c|c|c|c|c|c|c|c|}
\hline \multirow{3}{*}{$\begin{array}{c}\text { Hipp. } \\
\text { no. }\end{array}$} & \multirow{3}{*}{$\begin{array}{l}\text { HD } \\
\text { no. }\end{array}$} & \multirow{3}{*}{$\begin{array}{c}t_{\text {span }} \\
(\mathrm{yr})\end{array}$} & \multirow{3}{*}{ Start Date } & \multicolumn{4}{|c|}{ Number of RVs } & \multicolumn{4}{|c|}{ RMS of RVs $\left(\mathrm{ms}^{-1}\right)^{\mathrm{a}}$} \\
\hline & & & & \multicolumn{2}{|c|}{$\underline{\text { Lick Dewars }}$} & \multicolumn{2}{|c|}{ Keck Dewars } & \multicolumn{2}{|c|}{$\underline{\text { Lick Dewars }}$} & \multicolumn{2}{|c|}{$\underline{\text { Keck Dewars }}$} \\
\hline & & & & $\mathrm{p}$ & 1 & $\mathrm{k}$ & $\mathrm{j}$ & $\mathrm{p}$ & 1 & $\mathrm{k}$ & $\mathrm{j}$ \\
\hline 64394 & 114710 & 21.7 & $1987-06-13$ & 35 & 66 & $\cdots$ & $\cdots$ & 25.8 & 11.7 & $\cdots$ & $\cdots$ \\
\hline 64924 & 115617 & 23.2 & 1991-04-28 & 14 & 95 & $\cdots$ & 103 & 10.8 & 9.1 & $\cdots$ & 3.0 \\
\hline 67275 & 120136 & 23.7 & $1987-06-12$ & 24 & 99 & $\cdots$ & $\cdots$ & 92.7 & 24.9 & $\ldots$ & $\cdots$ \\
\hline 71284 & 128167 & 20.2 & 1988-03-04 & 18 & 36 & $\cdots$ & $\cdots$ & 53.4 & 51.1 & $\cdots$ & $\cdots$ \\
\hline 72659 & 131156 & 19.4 & $1995-02-21$ & $\cdots$ & 15 & $\cdots$ & 5 & $\cdots$ & 56.8 & $\cdots$ & 41.9 \\
\hline 73184 & 131977 & 14.8 & 1993-08-01 & 5 & 77 & $\ldots$ & $\ldots$ & 18.7 & 8.2 & $\ldots$ & $\ldots$ \\
\hline 75181 & 136352 & 7.2 & $2007-05-26$ & $\ldots$ & $\ldots$ & $\cdots$ & 23 & $\ldots$ & $\cdots$ & $\ldots$ & 4.4 \\
\hline 77257 & 141004 & 26.9 & 1987-09-09 & 16 & 149 & 8 & 81 & 16.1 & 10.2 & 2.7 & 6.2 \\
\hline 77760 & 142373 & 20.8 & 1987-09-10 & 34 & 61 & $\cdots$ & $\ldots$ & 16.8 & 8.8 & $\cdots$ & $\cdots$ \\
\hline 78072 & 142860 & 21.1 & 1987-06-11 & 27 & 30 & $\ldots$ & $\ldots$ & 56.7 & 19.2 & $\ldots$ & $\ldots$ \\
\hline 79672 & 146233 & 18.0 & 1996-07-19 & $\cdots$ & . & 27 & 79 & $\ldots$ & $\ldots$ & 3.7 & 6.2 \\
\hline 81300 & 149661 & 19.0 & 1991-04-28 & 14 & 67 & 31 & 3 & 14.7 & 10.4 & 7.6 & 9.6 \\
\hline 84862 & 157214 & 26.9 & 1987-09-08 & 25 & 71 & 8 & 42 & 10.5 & 7.8 & 1.6 & 3.4 \\
\hline 86974 & 161797 & 26.9 & 1987-09-10 & 29 & 132 & 13 & 40 & 13.1 & 8.5 & 3.7 & 4.4 \\
\hline 89962 & 168723 & 24.1 & 1987-09-10 & 17 & 81 & 47 & 27 & 10.6 & 7.8 & 14.5 & 5.9 \\
\hline 91438 & 172051 & 18.0 & 1996-07-19 & $\ldots$ & $\ldots$ & 32 & 36 & $\cdots$ & $\cdots$ & 4.0 & 3.0 \\
\hline 92043 & 173667 & 24.4 & 1987-06-11 & 31 & 56 & $\ldots$ & $\ldots$ & 89.3 & 76.0 & $\ldots$ & $\ldots$ \\
\hline 95447 & 182572 & 17.8 & $1996-10-10$ & $\cdots$ & $\cdots$ & 49 & 33 & $\ldots$ & $\ldots$ & 4.4 & 3.6 \\
\hline 96100 & 185144 & 23.2 & 1991-04-30 & 5 & 18 & 21 & 229 & 4.5 & 4.1 & 2.4 & 2.1 \\
\hline 96441 & 185395 & 10.2 & 2001-07-21 & $\ldots$ & 223 & $\ldots$ & $\ldots$ & $\cdots$ & 57.1 & $\ldots$ & $\ldots$ \\
\hline 98036 & 188512 & 27.2 & $1987-06-13$ & 28 & 155 & 7 & 20 & 8.0 & 7.4 & 3.9 & 3.9 \\
\hline 99461 & 191408 & 10.0 & 2004-08-20 & $\ldots$ & $\ldots$ & $\ldots$ & 45 & $\ldots$ & $\ldots$ & $\cdots$ & 2.3 \\
\hline 104214 & 201091 & 27.1 & $1987-06-11$ & 44 & 24 & $\cdots$ & 77 & 13.3 & 10.9 & $\ldots$ & 5.4 \\
\hline 104217 & 201092 & 27.1 & $1987-06-12$ & 30 & 26 & $\ldots$ & 74 & 17.0 & 11.7 & $\ldots$ & 7.0 \\
\hline 112447 & 215648 & 23.3 & 1988-06-19 & 23 & 77 & $\cdots$ & $\cdots$ & 17.3 & 11.9 & $\ldots$ & $\cdots$ \\
\hline 113357 & 217014 & 18.8 & $1995-10-12$ & $\ldots$ & 202 & $\ldots$ & 41 & $\ldots$ & 6.2 & $\ldots$ & 2.4 \\
\hline 114622 & 219134 & 21.9 & $1992-10-11$ & 11 & 35 & 1 & 113 & 11.8 & 6.4 & $\ldots$ & 7.8 \\
\hline 116771 & 222368 & 24.1 & $1987-09-08$ & 30 & 23 & $\cdots$ & $\ldots$ & 52.7 & 134.5 & $\cdots$ & $\cdots$ \\
\hline 14954 & 19994 & 24.1 & 1987-09-09 & 16 & 82 & $\ldots$ & $\ldots$ & 33.2 & 13.4 & $\ldots$ & $\ldots$ \\
\hline 18859 & 25457 & 11.1 & 2002-08-29 & $\ldots$ & $\ldots$ & 7 & 14 & $\ldots$ & $\cdots$ & 27.5 & 16.9 \\
\hline 29650 & 43042 & 10.9 & 2000-11-19 & $\ldots$ & 29 & $\cdots$ & $\cdots$ & $\cdots$ & 13.7 & $\ldots$ & $\ldots$ \\
\hline 32480 & 48682 & 27.0 & 1987-09-10 & 16 & 74 & $\cdots$ & 48 & 13.0 & 10.2 & $\ldots$ & 4.4 \\
\hline 39780 & 67228 & 12.8 & $1998-12-26$ & $\ldots$ & 39 & 16 & 21 & $\ldots$ & 11.8 & 16.5 & 8.9 \\
\hline 40843 & 69897 & 21.4 & 1987-09-10 & 23 & 46 & $\cdots$ & .. & 22.2 & 9.6 & $\ldots$ & 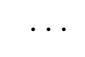 \\
\hline
\end{tabular}


This study is limited to Lick and Keck RVs gathered by the California Planet Search and its predecessor the California/Carnegie Planet Search. For a complete planet census and search completeness estimate, we urge other groups to undertake analyses for the historic Doppler measurements from the CFHT planet search (Walker et al. 1995), the AngloAustralian Planet Search (Tinney et al. 2001), the ELODIE, CORALIE, and HARPS planet searches (Mayor et al. 2011), the McDonald planet search (Wittenmyer et al.|2006), the Lick giant star planet search (Quirrenbach et al. 2011), and others.

\section{Doppler Planet Detection}

A planetary orbit is defined by an orbital period $P$ (or semi-major axis $a$ ), eccentricity $e$, inclination to the line of sight $i$, argument of periastron $\omega$, and longitude of the ascending node $\Omega$ (see e.g., Murray \& Dermott 1999). Imaging measurements of planetary (and stellar companions) consist of measuring the projected separation and position angle of the secondary relative to the primary source of light. Given a sufficient number, precision, and timing of such two-dimensional measurements, all five Keplerian orbital elements and the primary-secondary mass ratio can be inferred.

Doppler planet searches measure the line-of-sight velocity (radial velocity, RV) of the bright primary star and over time fit the measurements with a Keplerian model. Doppler measurements are sensitive to $P, e$, and $\omega$, but not $i$ or $\Omega$. They also are sensitive to the Doppler amplitude $K$ and an orbital reference time (typically a time of periastron passage, $t_{\mathrm{p}}$, or a time of transit, $\left.t_{\mathrm{c}}\right)$. For a planetary system with $n$ planets, the stellar reflex velocity is the sum of Keplerian contributions from each planet,

$$
v(t)=\sum_{j=1}^{n}\left(K_{j}\left(\cos \left(\omega_{j}+f_{j}(t)\right)+e_{j} \cos \omega_{j}\right)\right)+\gamma+\dot{\gamma} \cdot\left(t-t_{0}\right),
$$

where the term in big parentheses refers to the Keplerian signal from planet $j, f_{j}(t)$ is the true anomaly of planet $j$ at time $t, \gamma$ is the time-independent velocity offset (often degenerate with instrument-specific velocity zero points), $\dot{\gamma}$ describes a linear velocity term ("slope," "trend," or constant acceleration, often from a massive, secondary companion whose orbit is only partially sampled), and $t_{0}$ is a conveniently chosen epoch of the observations. The assumption in Eq. 1 that the total Doppler signal is the superposition of each planet's contribution implies that the planets are not dynamically interacting with one another. This is a good assumption because detectable planet-planet interactions require planets to be near or in mean-motion resonances, which have only been detected for a handful of Doppler systems (e.g., Rivera et al. 2010; Tan et al. 2013), and those systems can be discovered using their Keplerian approximations (Eq. 1). 
The true anomaly in Eq. 1 is defined implicitly in terms of the other three Keplerian parameters $P_{j}, t_{\mathrm{p}, j}$, and $e_{j}$ through the relations

$$
\begin{gathered}
\tan \frac{f_{j}(t)}{2}=\sqrt{\frac{1+e_{j}}{1-e_{j}}} \tan \frac{E_{j}(t)}{2}, \\
E_{j}(t)-e_{j} \sin E_{j}(t)=\frac{2 \pi\left(t-t_{\mathrm{p}, j}\right)}{P_{j}}=M_{j}(t) .
\end{gathered}
$$

Here $E_{j}$ is called the eccentric anomaly of planet $j, M_{j}$ is the mean anomaly, and Eq. 3 is Kepler's Equation. The Keplerian function $v(t)$ is sinusoidal for a single planet and $e=0$, but the function becomes significantly cusp-like as $e \rightarrow 1$ and the planet's orbital speed varies dramatically over one orbit (Figure 2). The RV semi-amplitude of the star, $K$, can be expressed in units of $\mathrm{m} \mathrm{s}^{-1}$ with the planet mass in units of Jupiter masses $\left(M_{\mathrm{J}}\right)$,

$$
K=\frac{28.4 \mathrm{~m} \mathrm{~s}^{-1}}{\sqrt{1-e^{2}}} \frac{M_{p} \sin i}{M_{\mathrm{J}}}\left(\frac{M_{\star}+M_{p}}{M_{\odot}}\right)^{-2 / 3}\left(\frac{P}{\mathrm{yr}}\right)^{-1 / 3},
$$

using Kepler's Third Law, $K \propto a^{-1 / 2}$. Thus, Doppler signals from giant planets in few AU orbits are of order $\sim 10 \mathrm{~ms}^{-1}$, while Neptune-mass planets and super-Earths produce 10-100× smaller signals. The timescales to discover a planet using Doppler velocities are daunting for $a$ larger than a couple AU; recall that Jupiter has $a=5.2 \mathrm{AU}$ and $P=11.9 \mathrm{yr}$ and Saturn has $a=9.6 \mathrm{AU}$ and $P=29.5 \mathrm{yr}$.

Also note that $K \propto M_{\mathrm{p}} \sin i$, the planet's "minimum mass," where $\sin i$ is in the range $0-1$ and accounts for the unknown orbital inclination $i$. For example, $i=45^{\circ}$ corresponds to $\sin i=1 / \sqrt{2}$ and the true mass $M_{\mathrm{p}}$ being $41 \%$ larger than $M_{\mathrm{p}} \sin i$. Large $\sin i$ corrections are rare because orbital orientations are randomly distributed on the celestial sphere. (The distribution of $\cos i$ is uniform.) The statistical probability that the orbit inclination is within a range $i_{1}<i<i_{2}$ is $P_{\text {incl }}=\left|\cos \left(i_{2}\right)-\cos \left(i_{1}\right)\right|$. This means that $87 \%$ of orbits have $30^{\circ}<i<90^{\circ}$, and $M_{\mathrm{p}}$ within a factor of two of $M_{\mathrm{p}} \sin i$. Randomly finding $i<30^{\circ}$ is equivalent to randomly selecting a location in the Northern Hemisphere with latitude $>60^{\circ}$, i.e. nearly in the Arctic Circle (latitude $=66.5^{\circ}$ ).

\section{Radial Velocity Analysis}

\subsection{Automated Planet Search}

We search for planets in the RV data using an iterative multi-planet detection algorithm based on the two-dimensional Keplerian Lomb-Scargle (2DKLS) periodogram (O'Toole et al. 
Table 3-Continued

\begin{tabular}{|c|c|c|c|c|c|c|c|c|c|c|c|}
\hline \multirow{3}{*}{$\begin{array}{c}\text { Hipp. } \\
\text { no. }\end{array}$} & \multirow{3}{*}{$\begin{array}{l}\text { HD } \\
\text { no. }\end{array}$} & \multirow{3}{*}{$\begin{array}{c}t_{\text {span }} \\
(\mathrm{yr})\end{array}$} & \multirow{3}{*}{ Start Date } & \multicolumn{4}{|c|}{ Number of RVs } & \multicolumn{4}{|c|}{ RMS of RVs $\left(\mathrm{ms}^{-1}\right)^{\mathrm{a}}$} \\
\hline & & & & \multicolumn{2}{|c|}{ Lick Dewars } & \multicolumn{2}{|c|}{ Keck Dewars } & \multicolumn{2}{|c|}{ Lick Dewars } & \multicolumn{2}{|c|}{ Keck Dewars } \\
\hline & & & & $\mathrm{p}$ & 1 & $\mathrm{k}$ & $\mathrm{j}$ & $\mathrm{p}$ & 1 & $\mathrm{k}$ & $\mathrm{j}$ \\
\hline 48113 & 84737 & 26.5 & $1987-06-13$ & 22 & 66 & $\ldots$ & 44 & 14.2 & 7.8 & $\ldots$ & 3.4 \\
\hline 53721 & 95128 & 27.1 & $1987-06-13$ & 19 & 215 & $\cdots$ & 48 & 15.5 & 8.2 & $\cdots$ & 2.9 \\
\hline 64408 & 114613 & 5.7 & $2007-05-26$ & $\ldots$ & $\ldots$ & $\ldots$ & 24 & $\ldots$ & $\ldots$ & $\ldots$ & 5.9 \\
\hline 64792 & 115383 & 21.7 & $1987-06-12$ & 21 & 16 & $\cdots$ & $\cdots$ & 28.8 & 23.6 & $\ldots$ & $\ldots$ \\
\hline 65721 & 117176 & 26.6 & 1988-02-03 & 21 & 98 & $\ldots$ & 54 & 9.5 & 9.1 & $\ldots$ & 3.5 \\
\hline 73996 & 134083 & 21.0 & $1987-06-11$ & 22 & 23 & $\ldots$ & $\cdots$ & 218.2 & 204.8 & $\ldots$ & $\ldots$ \\
\hline 97675 & 187691 & 11.1 & 2000-09-09 & $\ldots$ & 15 & $\ldots$ & $\ldots$ & $\ldots$ & 15.2 & $\ldots$ & $\ldots$ \\
\hline 109422 & 210302 & 15.1 & 1999-06-12 & $\ldots$ & $\ldots$ & 11 & 30 & $\ldots$ & $\ldots$ & 22.7 & 9.3 \\
\hline
\end{tabular}

aThe RMS of the RVs is computed as the standard deviation of the velocities after subtracting the signals discussed in Sec. 5.2 .

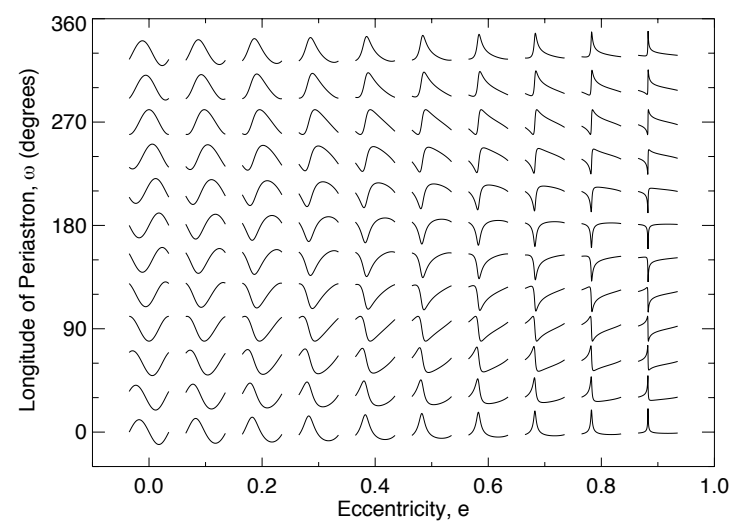

Fig. 2.- Keplerian function shape versus orbital eccentricity, e (0-0.9 in 0.1 steps), and longitude of periastron, $\omega\left(0-330^{\circ}\right.$ in $30^{\circ}$ steps $)$. This function represents the Doppler shift of a planet host star. It is sinusoidal for $e=0$, but becomes closer to a $\delta$-function as $e \rightarrow 1$. Orbits with $e \lesssim 0.4$ are reasonably well fit by single sinusoids, but more eccentric orbits are increasingly less likely to be detected by orbit fitting techniques that assume circular orbits. Each curve represents a Keplerian function with unit amplitude $(K)$, unit orbital period $(P)$, and a time of periastron passage $\left(t_{p}=t_{c}+P / 4\right.$ is shown, where $t_{c}$ is a time of transit), evaluated on the grid of $e$ and $\omega$. 
2009). We create the periodogram by fitting a Keplerian RV model to the dataset at many starting points on a two-dimensional grid of $P$ and $e$. The primary advantages of the 2DKLS periodogram over the Lomb-Scargle periodogram (Lomb 1976; Scargle 1982) are that it is more sensitive to eccentric planets, and both measurement errors and zero point offsets between instruments $\left(\gamma_{i}\right)$ can be incorporated directly into the periodogram.

We fit models to the data using the Levenberg-Marquardt (L-M) $\chi^{2}$-minimization in the RV fitting package (RVLIN, Wright \& Howard 2009). Each RV model is a sum of single planet models $\left(P, t_{\mathrm{p}}, e, \omega\right.$, and $K$ ), but with $\gamma_{i}$ (and $\dot{\gamma}$, if needed) shared by all planets (Eq. 1). We define a grid of search periods following the prescription of Horne \& Baliunas (1986) for optimal frequency sampling. At each period we start an L-M fit at five eccentricities between 0.05 and 0.7. All parameters are free to vary in each fit, but $P$ and $e$ are constrained to intervals that allow them to vary only half the distance to adjacent search $P$ and $e$ ranges in parameter space. All parameters for any previously detected planets are simultaneously re-fit so that slightly incorrect fits (sometimes caused by the presence of other planets) do not lead to the false detection of additional planets. The periodogram power is

$$
Z(P, e)=\frac{\chi^{2}-\chi_{B}^{2}}{\chi_{B}^{2}},
$$

where $\chi^{2}$ is the sum of the squared, error-normalized residuals to the current $N$-planet Keplerian fit, and $\chi_{B}^{2}$ is for the best $N$-1-planet fit. In the first iteration of the planet search (comparing 1-planet models to a 0-planet fit), $\chi_{B}^{2}$ is simply the sum of the squared, error-normalized residuals to the mean or a linear fit.

We start the iterative planet search by fitting for any known planets in the system using the orbital parameters cataloged in the Exoplanet Orbit Database (Wright et al. 2011) and well-established planet candidates (Howard et al. 2014 in prep.) as initial guesses for RVLIN. We then look for a significant linear trend by fitting a line to the RVs and checking if the total change in RV due to the fitted line is greater than 10 times the median of the individual measurement errors. If a significant trend is detected we allow $\dot{\gamma}$ to vary at each point in the 2DKLS periodogram, or if no trend is detected we fix $\dot{\gamma}=0$. For systems with known planets, we start by creating a periodogram to test if a $N+1$ planet model is a better fit than the model of $N$ known planets; otherwise we start by searching for a single planet and compare to the null hypothesis (the $N=0$ planet model). Note that for cases where the orbital period is between half and the full baseline of the dataset we can suffer a modest reduction in sensitivity. In the case of a partially sampled orbit the data may show an apparent linear trend that is partially absorbed into $\dot{\gamma}$. This effect dictates the smooth rolloff in sensitivity to long-period, low-mass planets as discussed in Section 5.5

Since RV measurement errors are often underestimated due to the presence of system- 


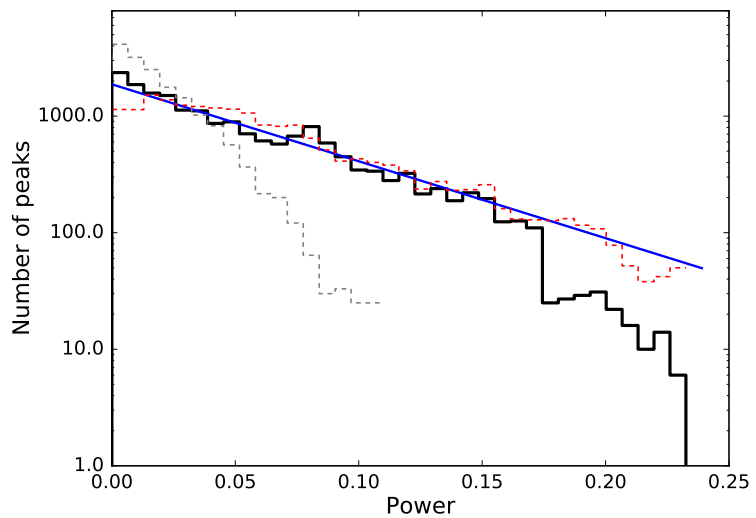

Fig. 3.- Distribution of 2KDLS periodogram peak heights for the HD 10476. (The periodogram is showing the top panel of Fig. 4.) We fit a linear profile to this distribution (blue line) and define the FAP $=1 \%$ threshold as the periodogram power where the linear extrapolation intersects $N=0.01$. As shown by the red line in the top panel of Fig. 4, this FAP threshold is at a power of $Z=0.85$, well separated from the forrest of peaks that have a maximum value of $Z=0.25$. The grey dashed line shows the periodogram value distribution for Gaussian noise, and the red dashed line shows the distribution for $1 / \mathrm{f}$ ("pink") noise simulation as described in $\$ 5.1$. It is interesting to note that our best fit model and the histogram of periodogram peaks for the real dataset more closely match the pure "pink" noise dataset then the "white" noise dataset. The histogram produced from the periodogram of "white" noise has much many more peaks at low power (note log y-axis) and drops off much faster with no periodogram peaks above a power of 0.1 in contrast to the observations. This indicates that the RVs for this star (and likely most stars) are dominated by non-Gaussian noise that arises from a combination of instrumental and astrophysical processes. 

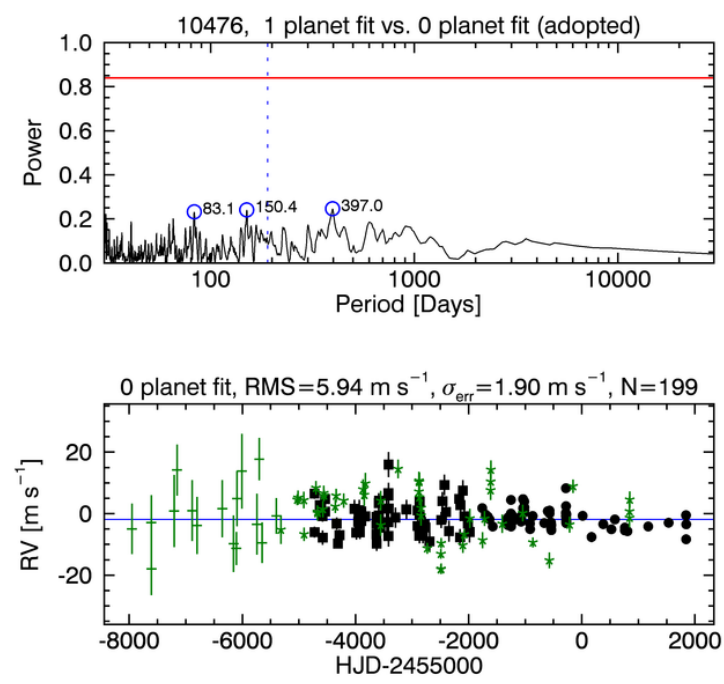

Fig. 4.- Two panel plot showing the results from an automated search for periodic signals in Lick and Keck RVs for the star HD 10476. Appendix C includes plots like this one for all stars without detected signals or Fig. 5 for stars with significant signals. Top panel: 2DKLS periodogram of the RVs from Lick and Keck. Periodogram power is calculated using Eq. 5 by comparing $\chi^{2}$ for prospective single planet fits having the range of periods on the horizontal axis to $\chi^{2}$ of the best-fit model without any planets (but with floating, telescope-specific RV zero points). The three most significant periodogram periods are labeled and circled in blue. In this case none of the peaks exceeds the $1 \%$ empirical FAP (solid red line) required for a solution to be adopted. The dashed vertical line marks the one year alias with the most significant peak (397 days). No planets were found significant in this fit, as indicated by "0 planet fit (adopted)" in the title of the top plot. Bottom panel: RVs as a function of time with $\gamma$ offsets between datasets minimized are plotted (the best-fit "0 planet fit"). The plot symbols are: green crosses (Lick "p"), green stars (Lick "l"), black squares (Keck "k"), black circles (Keck "j"). Annotations above the bottom panel indicate the RMS of the RVs, the median estimated Doppler uncertainty $\left(\sigma_{\text {err }}\right)$, and the number of RVs plotted $\left(N_{\text {obs }}\right)$. 

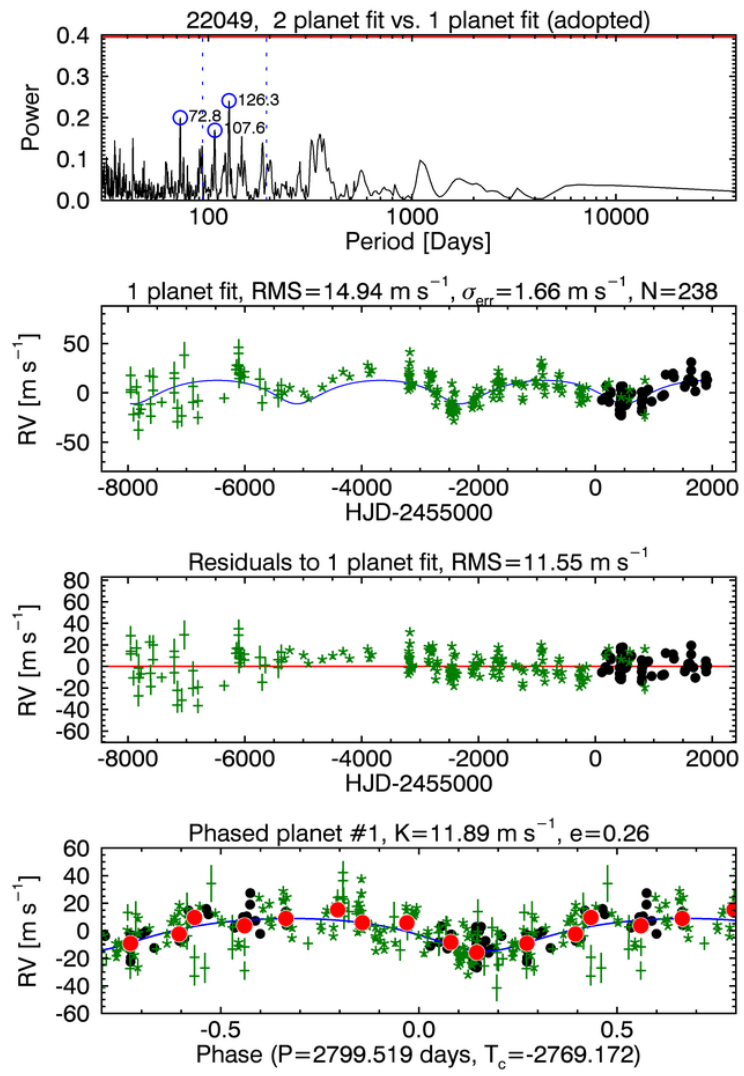

Fig. 5.- Four panel plot showing the results from an automated search for periodic signals in Lick and Keck RVs for the star HD 22049 ( $\epsilon$ Eridani; see Sec. 5.2 for a discussion). Appendix C includes plots of this type for all stars with detected planets. Top panel: 2DKLS periodogram of the RVs from Lick and Keck, as in Fig. 4. Periodogram power is calculated using Eq. 5 by comparing $\chi^{2}$ for prospective two-planet fits having the range of periods on the horizontal axis to $\chi^{2}$ of the best-fit single planet model. The three most significant periodogram periods are labeled and circled in blue. In this case none of the peaks exceeds the $1 \%$ empirical FAP (solid red line) required for a solution to be adopted. The dashed vertical lines mark the one year aliases with the most significant peak (126.3 days). The single planet fit is adopted, as indicated by "1 planet fit (adopted)" in the title of the top plot. Second panel: RVs as a function of time with the best-fit single planet model overplotted (blue line). Plot symbols are the same as in Fig. 4. Annotations above the bottom panel indicate the RMS of the RVs, the median estimated Doppler uncertainty $\left(\sigma_{\text {err }}\right)$, and the number of RVs plotted ( $N_{\text {obs }}$ ). Third panel: RV time series showing residuals to the best-fit single planet model in the second panel. The RMS of these residuals is indicated in the panel title. Bottom panel: RVs phased at the period of the adopted single planet model (2799 days). The panel title lists $K$ and $e$ for this planet. 
atic and/or poorly-understood astrophysical noise, periodogram power can not be directly converted into a significance estimate using traditional $\chi^{2}$ statistics. Instead we derive an empirical false alarm probability (FAP) by fitting a histogram of the periodogram amplitudes higher than the median power value to a linear function in $\log n$ vs. $Z$ (Figure 3 ). This provides an estimate of the number of peaks that should fall above a given value, and when multiplied by the number of independent test periods, the approximate probability that we would find a peak of a given value within the particular periodogram. We use this to define a threshold of $\mathrm{FAP}=1 \%$ above which spikes in the periodogram are marked as a planet candidates and we continue searching for the next potential planet in the system. If no significant signals are detected, we stop the search.

In order to compare an observed distribution of periodogram peak heights to some idealized cases we ran 2DKLS periodograms for simulated white and 1/f ("pink") noise datasets (see Figure 3). This was done by taking the real dataset for HD 10476 used to produce the plots in Figure 4 and replacing the velocities with Gaussian noise using sigmas equal to the measurement errors for each individual measurement. We then ran our 2DKLS periodogram on this dataset to measure the distribution of periodogram values in the case of pure white noise (grey dashed line in Figure 3). We also generated 1/f ("pink") noise using the Voss-McCartney algorithm (http://www.firstpr.com.au/dsp/pink-noise/) and scaled it to match the standard deviation of the real post-upgrade Keck RVs for the same star. We added this "pink" noise to the white noise dataset and ran the periodogogram again to measure the distribution of periodogram values in the case of pure white+pink noise (red dashed line in Figure 3).

If a periodogram peak is found to have an empirical FAP less than the $1 \%$ threshold some sanity checks are applied before it is considered a viable candidate. If greater than 20 peaks are found above the threshold we do not consider any of them viable unless the highest peak has an empirical FAP $\leq 10^{-4}$. This is caused by a lack of phase coverage in cases with a low number of observations $\left(N_{\text {obs }} \lesssim 20\right)$ and is not common among the generally well-observed stars studied in this work. If the period corresponding to the highest peak is within $10 \%$ of the periods of any previously found planets we mask this period and continue searching for additional periods until none are above the FAP threshold or a viable candidate is identified at a different period. Finally, if the L-M fit seeded at the period corresponding to the highest peak outputs a period that is $\geq 50 \%$ away from the input period we consider the period very poorly constrained and not a viable candidate.

Note that our empirical approach of estimating a FAP threshold differs from the bootstrapping strategy of scrambling RVs and searching for periodic signals in many realizations of the data, each with the RVs scrambled differently (e.g., Howard et al. 2010a). One ad- 
vantage of our FAP calculation here is that time-correlated RV noise is reflected in the distribution of periodogram peak heights, while the scrambling technique does not preserve that information.

See Figure 4 for an example of the automated planet search algorithm on the star HD 10476. The example shows data from all four instrument codes and the search did not detect any credible planetary signals. Figure 5 provides a second example, in this case a search for a second planet orbiting HD 22049 ( $\epsilon$ Eridani), which is known to host a single giant planet (Hatzes et al. 2000). A complete set of automated planet search plots for all stars in Table 1 is included in Appendix C.

\subsection{Known Signals in Doppler Data}

The automated planet searches for each star describe the completeness of our Doppler data in a search for planets in addition to the already known planets. That is, the completeness limits are for an $N+1$ planet model, compared to an $N$ planet model. We adopt the Exoplanet Orbit Database at exoplanets.org as the definitive source of "known planets" (Wright et al. 2011). In addition, we list below the other "known signals" (wide stellar binaries, activity correlations, etc.) that were seeded into our automated planet searches and that our completeness estimates are with respect to. We also list stars with interesting histories relevant to our RV planet searches. Not all stars with Doppler observations are listed below, only those with known signals/planets and a few for which we make comments.

HD 3651 (HIP 3093; program: $S$ ) Fischer et al. (2003) discovered an eccentric $(e=$ 0.63) sub-Saturn mass planet with $P=62$ day orbiting 54 Piscium. A $\mathrm{T}$ dwarf in a wide orbit was later discovered, making this the first known planetary system with an imaged brown dwarf (Mugrauer et al. 2006; Luhman et al. 2007; Liu et al. 2007). See Fig. C.2.

HD 4614 (HIP 3821; programs: $S, C, A)-\eta$ Cassiopeiae shows a significant linear trend with no detectable curvature, presumably due to its K7V stellar companion, HD 4614B ( $\eta$ Cassiopeiae B). See Fig. C.3.

HD 9826 (HIP 7513; programs: C, A) - v Andromedae hosts three known planets with masses of $0.67,1.9$, and $4.1 M_{\mathrm{J}}$ with orbital periods of 4.6, 241, and 1278 days (Butler et al. 1997, 1999; Wright et al. 2009). We detect an additional periodicity at $\sim 4000$ days and interpret this as the signature of a stellar magnetic activity cycle. See Fig. C.5.

HD 10700 (HIP 8102; programs: S, C, A)— $\tau$ Ceti is an extremely quiet RV standard star. Five low-mass planets were reported to orbit $\tau$ Ceti (Tuomi et al. 2013). However, we 
detect no periodic signals. The automated pipeline does detect a long-period signal but it appears to be caused by a poorly constrained offset between datasets. $\tau$ Ceti has long been an attractive planet search target because of its proximity $(3.7 \mathrm{pc})$ and brightness $(V=3.5)$. Frank Drake (1961) started modern SETI by searching for radio signals from $\tau$ Ceti and $\epsilon$ Eridani. See Fig. C.7.

HD 16160 (HIP 12114; program: S) - The RV time-series for this star shows significant curvature, likely from a late M-type companion (Golimowski et al. 1995a b; Tanner et al. 2010). The RV curvature makes it difficult to detect small, long-period planets $\left(P \gtrsim t_{\text {span }}\right)$ in this system. See Fig. C.9.

HD 17925 (HIP 13402; program: S) - The automated pipeline detects a marginally significant linear trend in the RV time series. See Fig. C.10.

HD 19994 (HIP 14954; program: A) - The star 94 Ceti hosts a giant planet in a $1.4 \mathrm{AU}$ orbit Mayor et al. (2004). See Fig. C.12.

HD 22049 (HIP 1653\%; programs: $C, A)-\epsilon$ Eridani is a young K2 dwarf that exhibits an RV period of $\sim 2500$ days, interpretable as a Jovian-mass planet (Hatzes et al. 2000). This star is one of the best prospects for directly imaging planets (Marengo et al. 2009) due to the star's proximity $(3.2 \mathrm{pc}$ ) and youth, and the detection of a debris disk (Greaves et al. 1998; Moran et al. 2004; Backman et al. 2009). The planet has remained controversial because of high stellar magnetic activity (Metcalfe et al. 2013; Jeffers et al. 2014). However, our measurements of the activity-sensitive Ca II H \& K lines (Isaacson \& Fischer 2010) have a period of $\sim 3$ yr and do not correlate with RVs spanning 6 yr in our Keck spectra. While such a correlation would have cast doubt on the planet, the absence of one strengths the case for a giant planet orbiting this star. See Fig. C.14.

HD 25457 (HIP 18859; program: A) - This young, F6 dwarf is a T Tauri star and a member of the AB Dor moving group (López-Santiago et al. 2006). Ground-based direct imaging searches for planets having yielded non-detections to date, with planet mass limits of $\sim 7 M_{\mathrm{J}}$ at $1 \mathrm{AU}$ (Maire et al. 2014). Our pipeline formally identifies a planet candidate based on 21 Keck RVs. Given the high jitter and small number of observations, we deem this candidate not credible with the current data. See Fig. C.17.

HD 30652 (HIP 22449; programs: $S, C, A)-\pi^{3}$ Orionis hosts no known planets. We detect a marginally significant periodic signal with a period of 338 days. However, the poor observing history and the proximity of the period to one year, we conclude that this signal is not caused by a real planetary companion. The nature of the signal is most likely non-astrophysical in nature. Wittenmyer et al. (2006) did not detect companions in their McDonald Observatory Doppler search. See Fig. C.20. 
HD 34411 (HIP 24813; programs: S, C, A) - The automated pipeline picks up a longperiod, high-eccentricity signal that is likely due to poorly constrained offsets between instruments for $\lambda$ Aurigae. See Fig. C.23.

HD 37394 (HIP 26779; program: S) - This young star is in the Pleiades association (López-Santiago et al. 2006) and shows a small, marginally significant linear trend. See Fig. C.24.

HD 48682 (HIP 32480; program: A) —56 Aurigae has a formally adopted signal that appears to be due to uncorrected zero-point offsets in the Lick RVs and not due a planet. See Fig. C.26.

HD 69830 (HIP 40693; program: S) — This star hosts three Neptune-mass planets (Lovis et al. 2006). However, the automated pipeline can only pick out the planets at 8.7 and 197 days. While we do not detect the 31.6 day period in the Lick or Keck data, we have not performed an analysis to show that our non-detection is dispositive. See Fig. C.28.

HD 75732 (HIP 43587; program: S) — 55 Cancri hosts five known planets (Fischer et al. 2009 ) with at least one that transits (Winn et al. 2011). The orbital periods are $P=0.74$, 14.6, 44, 260, and 5200 days. The middle three planets (b, c, and f) are approximately Saturn-mass while the innermost, transiting planet (e) is a super-Earth and the outermost planet $(d)$ is a super-Jupiter. See Fig. C.31.

HD 84737 (HIP 48113; program: A) - This star has a formally adopted signal that appears to be due to uncorrected zero-point offsets in the Lick RVs and not due a planet. See Fig. C.33.

HD 95128 (HIP 53721; program: A)—47 Ursae Majoris has hosts two well-known giant planets with semi-major axes of 2 and 3.6 AU (Fischer et al. 2002), and possibly a third planet at $\sim 11$ AU (Gregory \& Fischer 2010). Our automated search prefers a model with three planets, although the outer most planet has a poorly constrained orbit. See Fig. C.36.

HD 100623 (HIP 56452; program: S) - This star shows a significant linear trend with no detectable curvature. The Keck RVs $(\operatorname{code}=\mathrm{j})$ are very sparse and only 19 observations were collected in total for this star. See Fig. C.37.

HD 104304 (HIP 58576; program: S) - This system shows a strong long-term linear trend with curvature, likely due to a detected low-mass, stellar companion (Tanner et al. 2010; Schnupp et al. 2010). Our automated search prefers a model with a linear velocity trend (constant acceleration) in addition to the orbit segment from the companion (three bodies total), with considerable model degeneracy between the slope and the mass of the companion causing the RV curvature. See Fig. C.41. 
HD 115617 (HIP 64924; programs: S, C, A)-Vogt et al. (2010) reported three small planets orbiting 61 Virginis with $P=4.2,38$, and 124 days. We see evidence for only the first two planets in our Keck RVs. The inner planet $b$ is a small super-Earth with a mass of $5.3 \mathrm{M}_{\oplus}$ and planet $\mathrm{c}$ is approximately Neptune-mass $\left(19 \mathrm{M}_{\oplus}\right)$. See Fig. C.46.

HD 117176 (HIP 65721; program: A)—70 Virginis hosts a $\sim 7 M_{\mathrm{J}}$ planet in a $0.5 \mathrm{AU}$ orbit (Marcy \& Butler 1996). See Fig. C.47.

HD 120136 (HIP 67275; programs: S, C, A)— $\tau$ Bootis b was one of the first exoplanets discovered (Butler et al. 1997). It is an extreme hot Jupiter with a mass $6 M_{\mathrm{J}}$ and an orbital period of 3.3 days. We also detect a significant linear trend in the RV data and a periodicity at $\sim 5000$ days. However, due to the possibilities of offsets within the Lick data, it is difficult to trust a long-period signal in the Lick data alone (Fischer et al. 2014). The trend is likely real, but the 5000 day periodicity could be due to instrumental effects or a stellar magnetic activity cycle. See Fig. C.48,

HD 131156 (HIP 72659; program: S, C, A)- $\xi$ Bootis A is a young (200 Myr), late G star in a binary stellar system with the late K star, $\xi$ Bootis B (Mamajek \& Hillenbrand 2008) . The significant linear trend with no detectable curvature in the RV time series of $\xi$ Bootis A is likely due to the stellar companion. See Fig. C.50.

HD 131977 (HIP 73184; programs: S, C)—This star has a significant linear RV trend, likely due to a binary companion (Gl 570B). See Fig. C.51.

HD 161797 (HIP 86974; programs: S, C, A)-The RV time-series for $\mu$ Herculis shows a strong linear trend and significant curvature (or a possibly closed orbit). $\mu$ Herculis is a hierarchical triple stellar system. The data can be well-fit by a Keplerian orbit with a period of 9800 days, but this is very poorly constrained and the period is likely to be much longer. See Fig. C.60.

HD 168723 (HIP 89962; program: C) - The Lick data for $\eta$ Serpentis show a marginal linear trend while the long-baseline pre-upgrade Keck data does not show the trend. See Fig. C.61.

HD 185395 (HIP 96441; program: C, A) - This early-type star (F4 V) has high jitter and a claimed, controversial planet with $P \approx 150$ days (Desort et al. 2009). We see evidence in our Lick data for RV variation at this period and other periods related by the yearly alias, however these signals are not statistically significant. See Fig. C.66.

HD 191408 (HIP 99461; programs: S, C, A) - This star shows a slight linear trend with no detectable curvature, presumably due to its common proper motion companion LHS 487 (K4V). See Fig. C.69. 
HD 201091 (HIP 104214; programs: S, C, A) -61 Cygni A shows a strong linear trend caused by 61 Cygni B. Under the direction of Peter van de Kamp, Strand (1943, 1957) suggested that 61 Cygni A was orbited by one or two giant planets. These claims were later rejected by high precision RVs (Walker et al. 1995; Cumming et al. 2008). See Fig. C.70.

HD 201092 (HIP 10421\%; program: S) — 61 Cygni B shows a strong linear trend caused by 61 Cygni A. See Fig. C.71.

HD 217014 (HIP 11335\%; program: $S$ ) -51 Pegasi is the host to the first known exoplanet; a hot Jupiter with a mass of $0.5 M_{\mathrm{J}}$ and an orbital period of 4.2 days (Mayor \& Queloz 1995). We also detect a long-period signal but this is likely caused by instrumental offsets within the Lick dataset. See Fig. C.74.

HD 219134 (HIP 114622; programs: S, C) - This star has candidate planets including a giant planet in a $3 \mathrm{AU}$ orbit that we will continue to examine as more RVs are gathered.

\subsection{Injection-Recovery Completeness}

To determine our sensitivity to planets as a function of $M_{\mathrm{p}} \sin i$ and $P$ we injected synthetic planetary RV signals into the real RV data for each star, preserving the actual times of observation, the previously measured velocities, and the measurement uncertainties. We then used the iterative automated planet search algorithm (Sec. 5.1) to attempt to recover the injected signals. We injected planets on circular orbits uniformly distributed in $\log (K)$ and $\log (P)$ centered around the sensitivity threshold line determined from the method of Howard et al. (2010b). We did not study sensitivity as a function of eccentricity because $>80 \%$ of known Doppler-discovered planets have $e<0.4$ (Wright et al. 2011), for which a circular orbit approximation is adequate (Fig. 2).

Note that the completeness plots are a function of minimum mass, $M_{\mathrm{p}} \sin i$. While $M_{\mathrm{p}} \sin i$ gives a good estimate for $M$ usually, it is a significant underestimate for face-on orbits. These cases are rare though for the same reason that the fractional area of the Earths surface near the poles is small. For example, $87 \%$ of random inclinations are between 30 and 90 degrees (where the true mass is within a factor of two of the minimum mass). And there is only a 0.5 chance of $i<5.7^{\circ}$, where $M_{\mathrm{p}} \sin i$ underestimates $M$ by a factor of ten or more (Fischer et al. 2014).

We injected 5000 synthetic planets into the RV time-series for each star in the sample. The synthetic planets are injected in addition to any known planets in the system and the search algorithm starts by searching for one additional planet while simultaneously fitting 
for all known planets in the system. An injected planet is considered recovered if the highest peak in the periodogram is above the $1 \%$ FAP detection threshold, the period of that peak is within $25 \%$ of the injected period, and the phase of the recovered orbit is within $\pi / 6$ of the injected phase. In some cases multiple planets are recovered when only a single planet is injected. We still consider these cases good recoveries if any of the detected periods are within $25 \%$ of the injected period.

Completeness contours are derived from the injection-recovery tests by computing a two-dimensional moving average of the recovery rate over a $100 \times 100$ grid in semi-major axis (a) and $M_{\mathrm{p}} \sin i$ with an averaging window of width 0.5 dex. Figure 6 shows an example completeness plot. The full set of completeness contours for all stars in the sample are in Appendix C,

In some cases anomalies are visible in the shape of the completeness contours derived from the injection-recovery tests. A feature of enhanced sensitivity is sometimes seen at long periods. This is caused by stellar magnetic activity masquerading as the injected planet. Although we require that the period of the recovered planet is within $25 \%$ of the period of the injected planet and the recovered phase is within $\pi / 6$ of the injected phase, some of the injected planet signals will happen to look very similar to the magnetic activity signal. Planets that are well below the local completeness threshold will still be "recovered" when the algorithm finds the magnetic activity cycle as the most significant periodic signal and finds that it has the correct period and phase to match the injected signal. This situation can occur in $\lesssim 10 \%$ of cases and can corrupt the $16 \%$ and $84 \%$ completeness contours. In other cases narrow spikes of decreased sensitivity are seen well above the local completeness threshold. This occurs in systems with known planets. For example, see Fig. C.31. When a planet is injected at nearly the same period as a known planet, it can be absorbed by the fit for the known planets. Fortunately for the estimate, it is unlikely that two planets with very similar orbital periods could remain dynamically stable. Kepler-36bc has the smallest period ratio detected to date with $P_{c} / P_{b} \simeq 7 / 6$ (Carter et al. 2012).

The one-dimensional contours provided in machine-readable tables as a supplement to this report were derived by scanning the 2D completeness grid column-by-column starting at low then progressively higher $M_{\mathrm{p}} \sin i$ values until recovery rates of $16 \%, 50 \%$, and $84 \%$ are first reached. We report $M_{\mathrm{p}} \sin i$ as a function of $P, a$, and projected on-sky separation $(\theta)$ for each of the three 1D completeness lines which corresponds to the minimum $M_{\mathrm{p}} \sin i$ that is detectable in the given fraction of injections. In separate machine-readable files, we include the two-dimensional completeness for each star using the grid of semi-major axes and $M_{\mathrm{p}} \sin i$ values in the completeness plots. See Appendix A.

Appendix C includes completeness estimates for every star with RVs. 


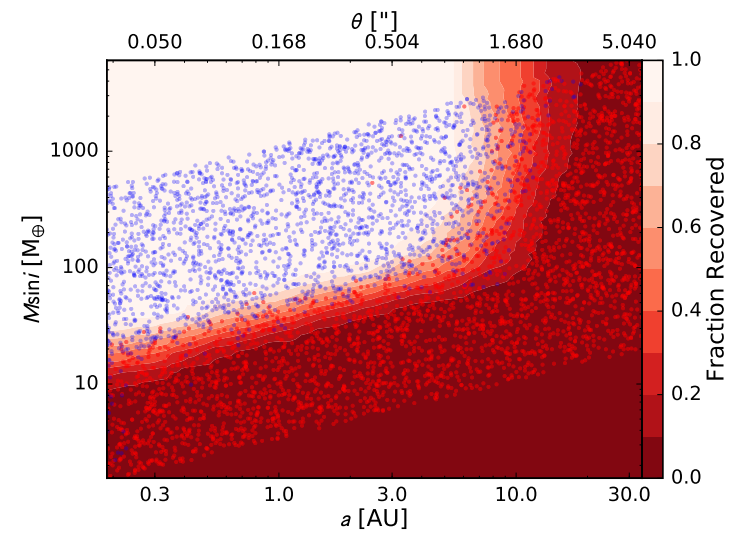

Fig. 6. - Example completeness plot for HD 4614 showing the results of our injection/recovery tests. Blue dots indicate the $M_{\mathrm{p}} \sin i$ and $a$ combinations of injected planets that were successfully recovered by our automated planet search, while red dots indicate injections that were not recovered. The contours are computed from a two-dimensional moving average over a $100 \times 100$ grid with window widths of 0.5 dex in $M_{\mathrm{p}} \sin i$ and $a$. Completeness in regions of the grid outside the limits of the injection-recovery tests are extrapolated vertically along columns from the nearest point in the grid that contains at least 20 injections within the averaging window. Appendix C includes a completeness plot for every star with Doppler measurements. 


\subsection{Summary Results}

Figures 7 and 8 summarize the results of our completeness estimates. Figure 7 shows the $50 \%$ detection thresholds for every star in the sample, with the median completeness contour shown in red. Note that the completeness contours span more than order of magnitude in $M_{\mathrm{p}} \sin i$, while having similar shapes. This variation is mostly due to the differing jitter values for stars observed in the Lick and Keck surveys. Figure 8 summarizes the full set of contours, slicing in both $M_{\mathrm{p}} \sin i$ and semi-major axis.

\subsection{Idealized Completeness}

An idealized Doppler planet search can be characterized by the number of observations $\left(N_{\text {obs }}\right)$, their time span $\left(t_{\text {span }}\right)$, and the precision of a single measurement $\left(\sigma_{\mathrm{RV}}\right)$. Precision stems from astrophysical noise sources ("jitter") that produce apparent Doppler shifts and measurements uncertainties stemming from the signal-to-noise ratio and the Doppler information content (number of lines, $v \sin i$, etc.) of each spectrum. In practice, $\sigma_{\mathrm{RV}}$ for a particular star can be empirically estimated as the standard deviation of the RVs measured over a long time span (assuming no orbital companions are present). That is, single measurement errors are approximately the measurement scatter. To improve sensitivity, several RVs of the same star are often gathered on a given night to average over stellar noise and to improve signal-to-noise. In such cases, $\sigma_{\mathrm{RV}}$ represents the scatter in the nightly averages (or the appropriate binning timescale).

In the limit of large $N_{\text {obs }}$, uniform orbital phase coverage (random times of observation), and an orbital period $P<t_{\text {span }}, 50 \%$ completeness can be characterized by a Doppler amplitude $K_{50}$ that is related to the survey parameters by

$$
K_{50}=\alpha \frac{\sigma_{\mathrm{RV}}}{\sqrt{N_{\mathrm{obs}}}}
$$

where $\alpha$ is a dimensionless parameter that represents the signal-to-noise ratio of a detectable plant, accounting for $\sqrt{N_{\text {obs }}}$ measurements. Naively, we expect $\alpha$ to be greater than one and of order a few. We estimated $\alpha$ using three techniques described below.

First, we estimated $\alpha$ using the completeness plots in Appendix C using $N_{\text {obs }}$ and $t_{\text {span }}$ from our measurements and taking $\sigma_{\mathrm{RV}}$ to be the RMS of the RVs after subtracting all significant signals. Using this technique we found $\alpha$ in the range $\sim 5-20$. This range accounts for the ways that real observations violate our assumptions of the idealized survey: telescope/instrument combinations have heterogenous Doppler precision (choosing the "typical" $\sigma_{\mathrm{RV}}$ difficult to estimate), RV zero point offsets between datasets must be fitted for, and 

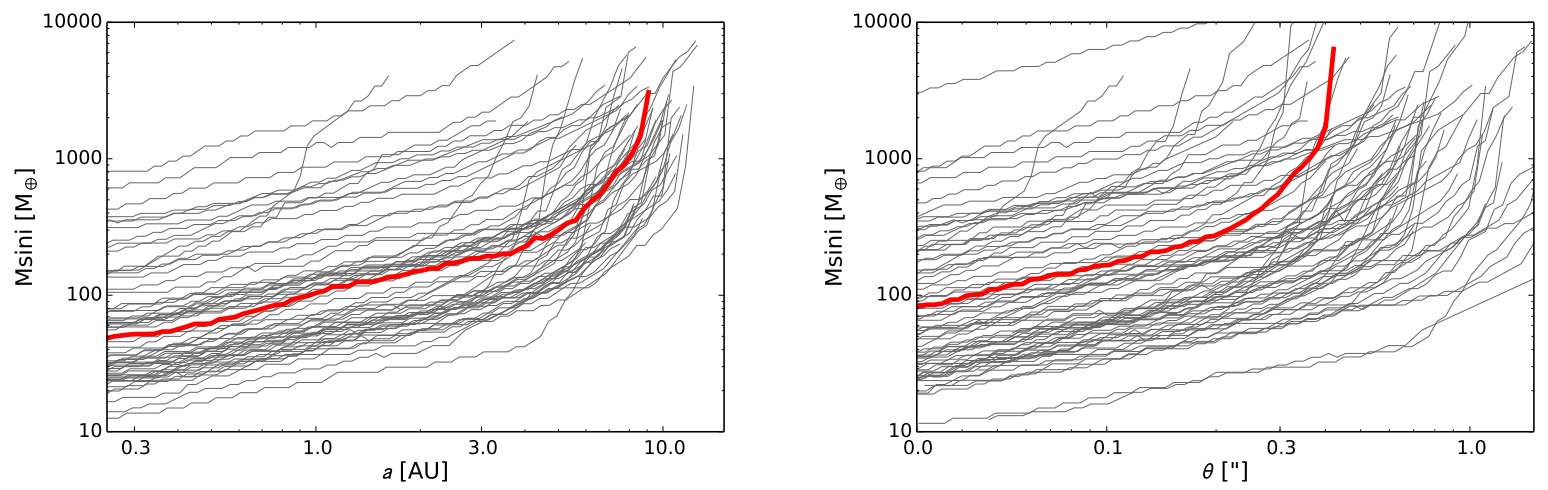

Fig. 7.- Contours showing 50\% completeness for every star with Doppler measurements (gray lines) and the median of those contours (red line). The panels show the same completeness curves as a function of semi-major axis (left) and projected separation (right).
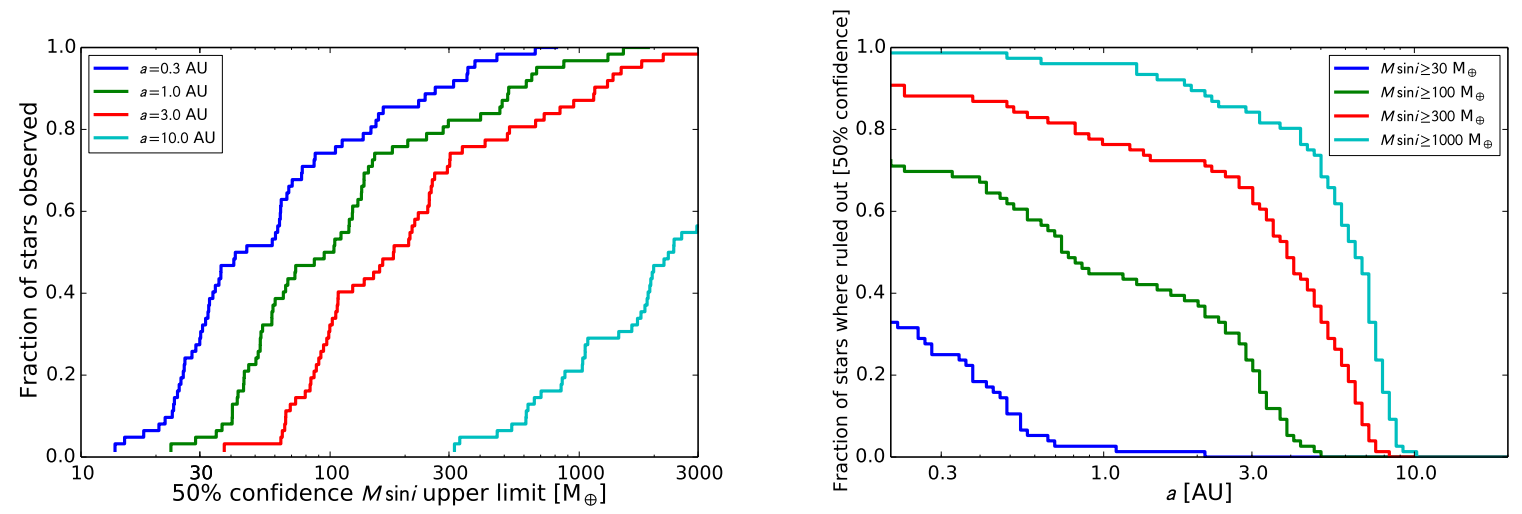

Fig. 8.- Fraction of stars observed by Lick/Keck Planet Searches whose RV observations can exclude planets of particular masses and semi-major axes. The left panel depicts the mass upper limits ( $M_{\mathrm{p}} \sin i$ at $50 \%$ confidence) at four semi-major axes while the right panel shows the fractions of stars with ruled out companions for four $M_{\mathrm{p}} \sin i$ values, as a function of semi-major axis. 
non-random observing cadences. Nevertheless, this factor of four range in $\alpha$ suggests that we might simulate completeness with similar mass precision simply by estimating survey and stellar parameters.

Our second method to estimate $\alpha$ was calculating completeness for an ideal observing campaign. Note in Eq. 6 that $K_{50}$ and $\sigma_{\mathrm{RV}}$ have the same units and scale linearly with one another. Searching for a $K=10 \mathrm{~m} \mathrm{~s}^{-1}$ signal with $\sigma_{\mathrm{RV}}=1 \mathrm{~m} \mathrm{~s}^{-1}$ precision is equivalent to searching for a $K=100 \mathrm{~m} \mathrm{~s}^{-1}$ signal with $\sigma_{\mathrm{RV}}=10 \mathrm{~m} \mathrm{~s}^{-1}$. Making the problem dimensionless, we define a survey sensitivity,

$$
\kappa_{50}=\frac{K_{50}}{\sigma_{\mathrm{RV}}}=\frac{\alpha}{\sqrt{N_{\mathrm{obs}}}}
$$

that is valid for dimensionless orbital periods $\tau=P / t_{\text {span }} \lesssim 1$.

We tested and calibrated this idealized completeness model with injection-recovery simulations. Figure 9 shows the simulation results and their parameters, with the plots demonstrating that $\kappa \propto K /\left(\sigma_{\mathrm{RV}} \cdot \sqrt{N_{\mathrm{obs}}}\right) . \kappa_{50}(\tau)$ is flat in these three simulations for short period orbits. Based on these simulations, we estimate $\alpha \approx 6$. For $\tau \gtrsim 1.5$, the $\kappa_{50}$ contour rises rapidly; we estimate a slope of $\sim 10$ and $\kappa_{50}(\tau)=\alpha \cdot 10^{\tau-1.5} / \sqrt{N_{\text {obs }}}$. We can combine these two functions smoothly using quadrature addition,

$$
\kappa_{50}(\tau)=\frac{\alpha}{\sqrt{N_{\mathrm{obs}}}} \cdot \sqrt{1+\left(10^{\tau-1.5}\right)^{2}}
$$

giving

$$
K_{50}(\tau)=\frac{\sigma_{\mathrm{RV}} \alpha}{\sqrt{N_{\mathrm{obs}}}} \cdot \sqrt{1+\left(10^{\tau-1.5}\right)^{2}}
$$

Eq. 9 nicely encapsulates the $50 \%$ percentile search completeness as a function of a three observational parameters: $\sigma_{\mathrm{RV}}, N_{\mathrm{obs}}$, and $t_{\text {span }}$. However, the simplistic form is only a fit to an idealized observational campaign and is likely accurate to a factor of a few (perhaps good enough for planning observations). Real life observing is more complicated. One should consider the following caveats when applying this idealized completeness model:

1. Measurement errors are rarely Gaussian distributed. Velocity errors from stellar jitter and instrument systematics are often time correlated. Periodic astrophysical noise sources (i.e., magnetic activity cycles) can masquerade as planets and/or the hinder detectability of small planets. Thus, $\sigma_{\mathrm{RV}}$ doesn't fully encapsulate the Doppler noise sources.

2. Times of observation are non-random, depending on daily, monthly, and yearly cycles, not to mention scientific interests and the vicissitudes of telescope time allocation 

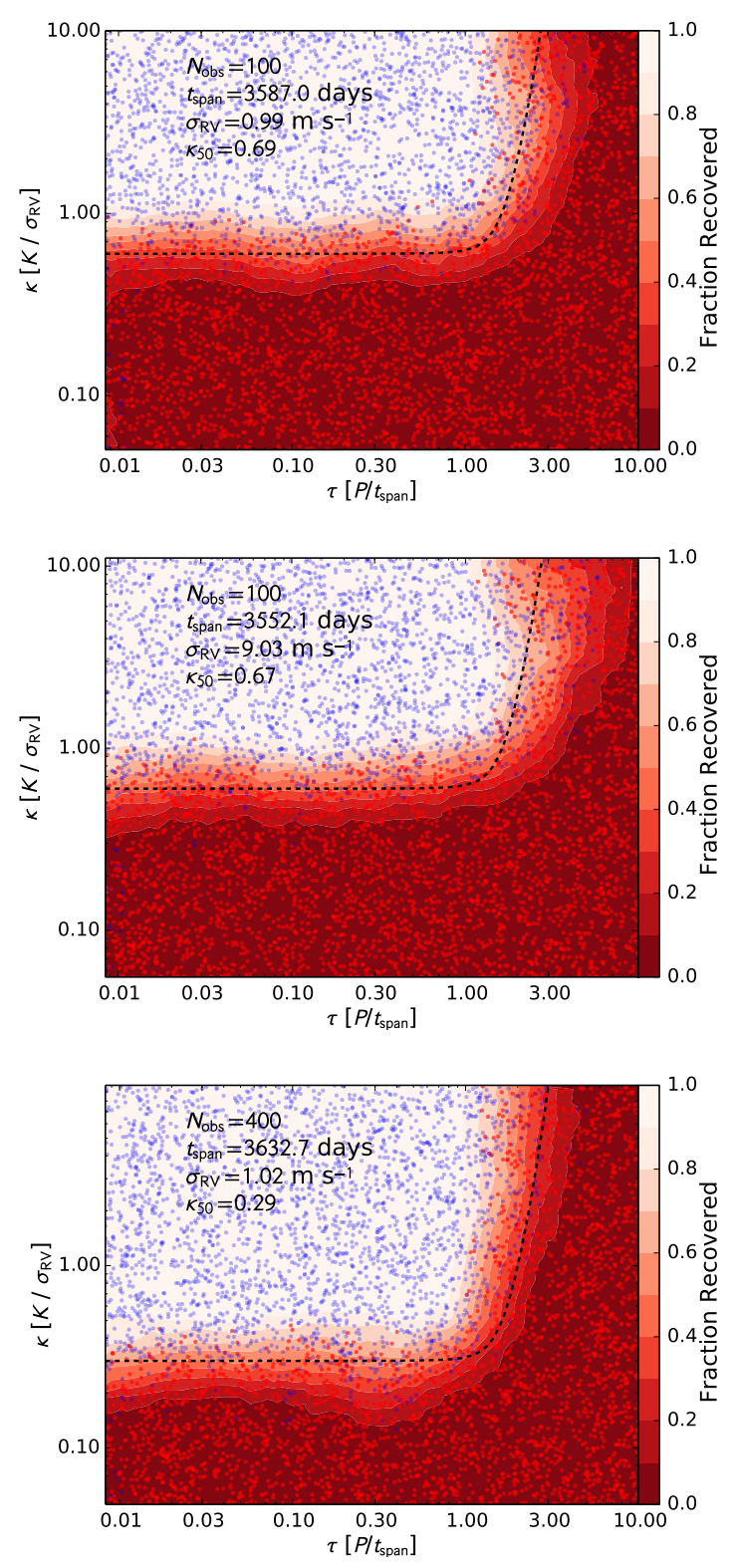

Fig. 9.- Planet search completeness in dimensionless Doppler amplitude $\kappa=K / \sigma_{\mathrm{RV}}$ versus dimensionless orbital period $\tau=P / t_{\text {span }}$ for three idealized observational surveys. The survey parameters are: $N_{\mathrm{obs}}=100, \sigma_{\mathrm{RV}}=1 \mathrm{~m} \mathrm{~s}^{-1}, t_{\mathrm{span}}=10 \mathrm{yr}(\mathrm{top}) ; N_{\mathrm{obs}}=100, \sigma_{\mathrm{RV}}=10$ $\mathrm{m} \mathrm{s}^{-1}, t_{\text {span }}=10 \mathrm{yr}$ (middle); $N_{\mathrm{obs}}=400, \sigma_{\mathrm{RV}}=1 \mathrm{~m} \mathrm{~s}^{-1}, t_{\mathrm{span}}=10 \mathrm{yr}$ (bottom). The three surveys give consistent $\alpha=\kappa_{50} \cdot \sqrt{N_{\mathrm{obs}}} \approx 6$ for $\tau<1$, showing that idealized completeness is scale invariant (see caveats in Sec. 5.5). Dashed black lines show $\kappa_{50}(\tau)$ estimated from Eq. 8. 
committees. Our assumption of uniform phase coverage (from random times of observation and large $N_{\text {obs }}$ ) is rarely achieved for real surveys. Non-randomly timed clusters of observation times inject period aliases into the periodograms, which can suppress or enhance the detectability at particular periods (Dawson \& Fabrycky 2010). Furthermore, combining RVs from multiple telescopes can reduce sensitivity to long-period signals since such signals can be absorbed into the fitted zero-point offsets.

3. Completeness estimates using quantified detection criteria often fail to account for human evaluation of data. Many observers are cautious about announcing planet discoveries, having seen instrumental systematics and correlated astrophysical noise exceed their prior expectations.

Our third method of estimating $\alpha$ involves examining the parameters of known planets on exoplanets.org (Wright et al.2011). Figure 10 shows estimates of $\kappa$ and $\alpha$ for planets with Doppler-measured masses with 100 pc. Only a handful of planets having been discovered with $\kappa<1$, i.e. a signal smaller than the noise. Such planets include HD 85512b (Pepe et al. 2011) and HD 156668b (Howard et al. 2011a). Our estimate of $\alpha \gtrsim 6$ for successful detection based on injection-recovery simulations appears to be slightly optimistic compared to the distribution of discovered planets that have a discovery threshold of $\alpha \gtrsim 10$ more commonly. Note that Fig. 10 plots $\kappa$ and $\alpha$ that are defined slightly differently than in Eq. 7 and 9. The figure shows the Doppler amplitude $K$, not the $50 \%$ completeness value $K_{50}$. And $N_{\text {obs }}$ is the number of measurements in the "orbit reference" paper (ORBREF on exoplanets.org), not the number of measurements when the planet was first detectable. Still, the discovery of some exoplanets with $\alpha \approx 10$ suggests that our estimate of $\alpha \approx 6$ is not wildly off.

\subsection{Prospective Completeness for Unobserved Stars}

For stars without Doppler observations, it is often helpful to estimate the sensitivity of a prospective Doppler campaign. With the above caveats in mind, we can use the idealized completeness formalism of Sec. 5.5 to estimate completeness. The campaign is characterized by $N_{\text {obs }}$ and $t_{\text {span }}$, while the the RV observations are characterized by $\sigma_{\mathrm{RV}}$. The general procedure to estimate the $50 \%$ completeness in mass as a function of semi-major axis, $M_{p} \sin i_{50}(a)$, is:

1. Choose $N_{\text {obs }}$ and $t_{\text {span }}$ for the survey. Choose a star with a particular $M_{\star}$ and estimate $\sigma_{\mathrm{RV}}$ from the expected jitter and measurement uncertainties. 

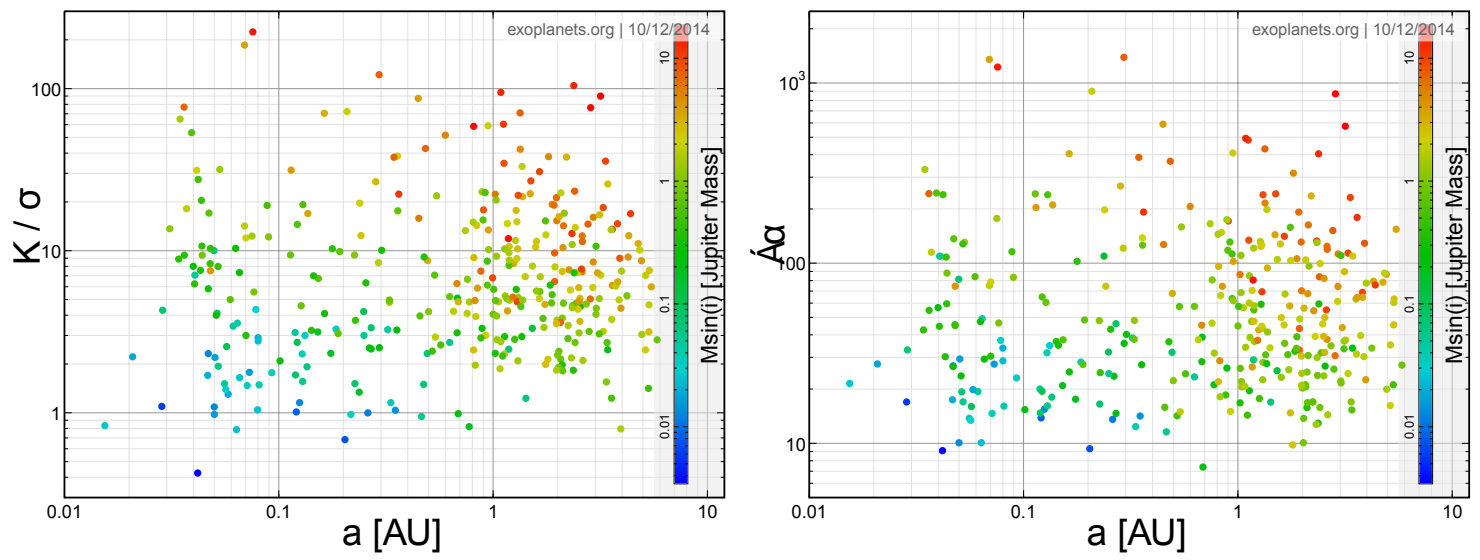

Fig. 10.- Distributions of $\kappa=K / \sigma_{\mathrm{RV}}$ (left) and $\alpha=\sqrt{N_{\mathrm{obs}}} K / \sigma_{\mathrm{RV}}$ (right) vs. semimajor axis $a$ for real planets within 100 pc with Doppler-measured masses on exoplanets.org (Wright et al. 2011). The plot includes discoveries from HIRES, HARPS, and other facilities. Point color indicates $M_{\mathrm{p}} \sin i$.

2. Compute $K_{50}(P)$ from Eq. 9 using $\alpha=6$ (idealized simulations) or $\alpha=10$ (the threshold of detection for historical discoveries).

3. Convert $K_{50}(P)$ into a $50 \%$ mass contour $M_{p} \sin i_{50}(P)$ using the inverted form of Eq. 4 for circular orbits,

$$
\frac{M_{p} \sin i_{50}(P)}{M_{\mathrm{J}}}=\frac{K_{50}(P)}{28.4 \mathrm{~m} \mathrm{~s}^{-1}}\left(\frac{M_{\star}+M_{p}}{M_{\odot}}\right)^{2 / 3}\left(\frac{P}{\mathrm{yr}}\right)^{1 / 3} .
$$

For planetary mass companions $\left(M_{\star} \gg M_{p}\right)$, the first term in parentheses simplifies to $\left(M_{\star} / M_{p}\right)^{2 / 3}$.

4. Convert $P$ to $a$ using Kepler's Third Law, $a=\left(G M_{\star} P^{2} / 4 \pi^{2}\right)^{1 / 3}$, again assuming $M_{\star} \gg M_{p}$.

Computing idealized completeness for all unobserved stars (Table B.1) requires good estimates of $M_{\star}$ and $\sigma_{\mathrm{RV}}$ for every star. $M_{\star}$ could be reasonably estimated from photometry and other sources, but the expected RV scatter, $\sigma_{\mathrm{RV}}$, is difficult to estimate accurately without a detailed study of each star. In lieu of detailed completeness estimates for every star in Table B.1, we provide the above recipe for estimating completeness with due caution that the $M_{p} \sin i_{50}(P)$ limits scale linearly with $\sigma_{\mathrm{RV}}$. Underestimated astrophysical and/or measurement errors will give false hope for planet detectability. 
Stars were not included in our historical Lick/Keck Doppler search for several reasons enumerated in Table B.1. These include: the star is too far South for Keck/Lick; the star has evolved into a subgiant or giant; the star is earlier spectral type than $\sim \mathrm{F} 8 \mathrm{~V}$; the star is young and chromospherically active; and the star is a spectroscopic binary. We discuss the expected Doppler precision $\sigma_{\mathrm{RV}}$ for each class below.

Southern Hemisphere-Many excellent main sequence G and K dwarfs are simply too far South to be easily observed at Keck and/or Lick Observatory (the cutoff is $-30^{\circ}$ to $-40^{\circ}$ declination). Observations of these stars could yield completeness curves comparable to our best cases in Fig. 7. Other teams are likely observing many of these stars (e.g., Mayor et al. 2011).

Early Spectral Type - Spectral types earlier than $\sim$ F8V have a decreasing density of spectral lines and an increasing average $v \sin i$, both of which degrade Doppler precision to $\gtrsim 5 \mathrm{~m} \mathrm{~s}^{-1}$ for the sample identified for Lick and transferred to Keck. Galland et al. (2005) developed a Doppler planet search for A-F stars. They measured the RV scatter for a sample of stars observed with ELODIE and HARPS finding an RV scatter of $\sigma_{\mathrm{RV}} \approx 0.16 \times v \sin i^{1.54}$ (for ELODIE observations with $\mathrm{S} / \mathrm{N}=200$ ). This relationship provided an accurate precision forecast at the factor of two level. The $v \sin i$ scaling to the $\sim 1.5$ power is consistent with a study by Bouchy et al. (2001) on the fundamental photon noise limit to radial velocity measurements, in the case of early $\mathrm{F}$ type main sequence stars. In the examples below, we adopt the Galland et al. (2005) prescription for $\sigma_{\mathrm{RV}}$. Note however that this relation fails for late $\mathrm{A}$ and early $\mathrm{F}$ type stars $(B-V$ between 0.2 and 0.4 ) that are often highly RV variable. This $B-V$ range corresponds to the intersection of the instability strip and the main sequence, where pulsators including $\delta$ Scuti and $\gamma$ Doradus stars are found. The most massive stars in the Exo-C/Exo-S/WFIRST-AFTA target lists are $\mathrm{B}$ stars $(B-V<0$; Fig. 1). Doppler measurements have achieved $\sigma_{\mathrm{RV}}=0.8$ to $2.0 \mathrm{~km} \mathrm{~s}^{-1}$ for such targets, depending on $v \sin i$ (J. Johnson, personal communication).

Evolved Stars - Surface gravity is reduced as stars evolve off the main sequence. The resulting surface oscillations produce RV variations that can be used to measure precise stellar properties (asteroseismology), and that serve as a noise source for Doppler planet searches. Kjeldsen \& Bedding (1995) estimated the Doppler oscillation amplitude, finding a dependence on the light-to-mass ratio, $v_{\text {osc }}=0.234\left(L_{\star} / M_{\star}\right) \mathrm{m} \mathrm{s}^{-1}$. We adopt this estimate of $v_{\text {osc }}$ for $\sigma_{\mathrm{RV}}$ for giant stars. As a check, this formula predicts $v_{\mathrm{osc}}=6 \mathrm{~m} \mathrm{~s}^{-1}$ for Pollux and Reffert et al. (2006) observed an RV scatter of $9 \mathrm{~m} \mathrm{~s}^{-1}$, which is the level of agreement we expect for estimates like these.

Young Stars - Line shape distortions due to rotationally modulated stellar surface features (e.g., spots and plage) make young stars difficult targets for planets searches (Crockett 
et al. 2012). Hillenbrand et al. (2014) measured the RV scatter for a set of young stars observed by HIRES, finding $\sim 100 \mathrm{~m} \mathrm{~s}^{-1}$ scatter for young stars $(\sim 30 \mathrm{Myr})$ with the activity measure $\log R_{\mathrm{HK}}^{\prime}=-4.0$, and RV scatter decreasing approximately linearly with $\log R_{\mathrm{HK}}^{\prime}$ to $\sim 3 \mathrm{~m} \mathrm{~s}^{-1}$ for old, quiet stars with $\log R_{\mathrm{HK}}^{\prime}=-5.0$.

Spectroscopic Binaries - Spectroscopic binaries are routinely excluded from Doppler surveys because their spectra cannot be modeled by standard cross-correlation or forwardmodeling techniques. However, techniques developed by Konacki et al. (2009) show promise for detecting $\sim M_{\mathrm{J}}$ planets for close-in orbits and several $M_{\mathrm{J}}$ planets for $\sim \mathrm{AU}$ orbits. We decline to provide specific advice for the expected Doppler precision and completeness for these stars since such estimates would be highly uncertain.

Using the above estimates for $\sigma_{\mathrm{RV}}$, we computed prospective completeness curves for eight stars that are representative of the imaging targets that lack Doppler measurements. These idealized completeness curves are based on a hypothetic survey with $n_{\mathrm{obs}}=100$ and $t_{\mathrm{span}}=10 \mathrm{yr}$ and $\sigma_{\mathrm{RV}}$ dependent on stellar characteristics. See Figure 11, which includes four early type stars ( $\beta$ Leo, A3V; $\rho$ Gem, F0V; HD 195689, A0V; RR Lyr, F0V, pulsator), a quiet dwarf in the Southern Hemisphere (p Eri A, K0V), and and the three brightest giant stars in Table B.1 (Arcturus, K0III; Aldebaran, K5III; Pollux, K0III). These simulations suggest that giant planets orbiting giant stars in $\sim \mathrm{AU}$ orbits can be detected by dedicated Doppler campaigns, as indeed they have been (e.g., Quirrenbach et al. 2011; Trifonov et al. 2014). Estimating completeness for all stars in Table B.1 can be obtained by applying the formulas for $\sigma_{\mathrm{RV}}$ (above) or measuring it directly (preferred), estimating $M_{\star}$ for each star, and carefully identifying pulsating and young stars that will be particular unpredictable.

\section{Recommendations for Future Doppler Surveys}

\subsection{Limits to Doppler Precision}

The Doppler uncertainty of a single measurement is often expressed as the quadrature sum of three terms: stellar jitter, Poisson uncertainty stemming from the signal-to-noise ratio of the spectrum and the information content in spectrum (Bottom et al. 2013), and instrumental uncertainty.

Next-generation Doppler instruments are pushing for $\leq 0.5 \mathrm{~m} \mathrm{~s}^{-1}$ precision (e.g., Pepe et al. 2014) with the ultimate goal of reaching $<0.1 \mathrm{~m} \mathrm{~s}^{-1}$ precision (Pepe \& Lovis 2008; Pasquini et al. 2010). These are engineering challenges that will only bear fruit if the stars themselves are quiet enough for planet detection at those levels. 
Stellar jitter represents a major challenge for Doppler planet detection. All stars except for old $\mathrm{G}$ and $\mathrm{K}$ dwarfs have jitter that precludes the detection of small planets in few AU orbits (Fig. 11). There are many sources of jitter. Acoustic oscillations including p-modes cause the stellar surface to oscillate with a characteristic timescale of a few minutes and a characteristic amplitude of $\sim 1 \mathrm{~m} \mathrm{~s}^{-1}$ for Solar-type stars. The convective overturning of granules on the stellar surface (granulation) changes the flux balance between sinking cool regions and rising hot cells, producing a jitter of a similar scale. These effects can be mitigated by observational strategies that average over the relevant timescales with multiple RV measurements taken per night (Dumusque et al. 2011a. b). Jitter from rotationally modulated stellar surface features (spots, plage, faculae) cause apparent Doppler shifts due to distorted line profiles at the $\sim 1 \mathrm{~m} \mathrm{~s}^{-1}$ level even for quiet stars. These signals can be mitigated by measuring stellar activity proxies and de-correlating the RVs (Dumusque et al. 2011c). In some sense, these short-term jitter signals are less troublesome for searches for long-period planets. Short-timescale jitter sets an error floor for single measurements, but multiple measurements on the appropriate timescales can average over these noise sources.

Long-term magnetic cycles analogous to our Sun's activity cycle pose perhaps the greatest challenge in the search for long-period planets (Isaacson \& Fischer 2010; Lovis et al. 2011). Coherent, nearly sinusoidal Doppler shifts of a few $\mathrm{m} \mathrm{s}^{-1}$ amplitude with 5-20 yr periods are common even for "inactive" $\mathrm{G}$ and $\mathrm{K}$ dwarfs and have led to a small number of apparent false planet claims. For example, one of the best Jupiter analog exoplanets is HD $154345 \mathrm{~b}$ (Wright et al. 2008), whose existence has been questioned because of a strong correlation between the RVs and the $S_{\mathrm{HK}}$ measure of stellar activity. Current Doppler planet searches routinely monitor activity diagnostics, including the Ca II H \& K lines (from which the $S_{\mathrm{HK}}$ values are derived) and the width and bisectors of the spectrum's cross correlation function. Figure 12 shows two example stars form our Keck-HIRES planet search. Both are "inactive stars." The top star (HD 14412) has a $K \approx 3 \mathrm{~ms}^{-1}$ signal with $P \approx 6 \mathrm{yr}$, the signature of an apparent Saturn-mass planet. This signal is mirrored in the $S_{\mathrm{HK}}$ time series, which has the same period and phase as the RVs. The bottom star (HD 23439) is even less active and has no detectable variation in $S_{\mathrm{HK}}$, and a reduced overall jitter.

Doppler planet searches are pushing the sensitivity limits by modeling and subtracting jitter, de-correlating it, selecting spectral lines and regions less sensitive to jitter, and selecting low-jitter stars for searches. Some groups are developing techniques to suppress stellar activity including modeling magnetic activity with time-series methods (Haywood et al. 2014: Grunblatt et al. 2015), identification and removal of photospheric signals (Hébrard et al. 2014), and Bayesian methods to identify noise by its temporal characteristics. For imaging search to take advantage of Doppler searches with $K \lesssim 0.5 \mathrm{~m} \mathrm{~s}^{-1}$ sensitivity, these developing techniques must be incorporated. 


\subsection{Ten Year Forecast}

We estimated the improvement in completeness that could be produced by continuing our Doppler observations for the next ten years. This estimate only assumes current techniques to identify low-jitter stars and to obtain RVs at $0.5 \mathrm{~m} \mathrm{~s}^{-1}$ precision. Future jitter-suppressing techniques could improve planet sensitivity further. Figure 13 shows our current completeness estimates (top row) for two stars observed at Keck (but not Lick). HD 102365 (left column in Figure 13) is one of the most poorly observed stars in our sample with only $N_{\text {obs }}=16$ measurements spanning the last $6.6 \mathrm{yr}$ with an RMS of $2.5 \mathrm{~m} \mathrm{~s}^{-1}$. (It has been poorly observed because it is relatively far South, $\delta \simeq-40^{\circ}$.) HD 182572 (right column in Fig. 13) has $N_{\text {obs }}=82$ measurements spanning $17.8 \mathrm{yr}$ with an RMS of $4.0 \mathrm{~m} \mathrm{~s}^{-1}(3.6$ $\mathrm{ms}^{-1}$ for HIRES instrument code $\mathrm{j}$ ).

The Fig. 13 plots also show the results of two simulations. In the middle row, we simulated adding $N_{\mathrm{obs}}=30$ measurements over $10 \mathrm{yr}$ to the existing RVs with $\sigma_{\mathrm{RV}}$ of the new measurements equal to the RMS of the most recent HIRES RVs (code $=\mathrm{j}$ ). This simulation represents the improvement in completeness from continuing observations with the current precision and a cadence of $N_{\text {obs }}=3$ RVs per year.

The bottom row of Fig. 13 shows the gain in completeness resulting from improved Doppler precision. Here we adopted $\sigma_{\mathrm{RV}}=0.5 \mathrm{~m} \mathrm{~s}^{-1}$ with $N_{\mathrm{obs}}=100$ over $10 \mathrm{yr}$. This level of precision is possible, but on the cutting edge of current Doppler techniques. It requires the most Doppler quiet stars - old, choromospherically inactive late-G and early-K dwarfsand observational strategies that average over stellar noise. To date, the lowest noise RV planet detections have been with HARPS. For example, HD 88512 b was detected with RVs whose per-night measurement uncertainties are $<0.3 \mathrm{~m} \mathrm{~s}^{-1}$ and the data set has an RMS of $0.75 \mathrm{~m} \mathrm{~s}^{-1}$ after subtracting the single planet model with $K=0.77 \mathrm{~m} \mathrm{~s}^{-1}$ (Pepe et al. 2011). Achieving this level of precision would enable $50 \%$ completeness to $\sim 10 M_{\oplus}$ planets (typical for super-Earths) at $3 \mathrm{AU}$, a factor of $\sim 6$ improvement in mass sensitivity compared adding $N_{\text {obs }}=30$ RVs over $10 \mathrm{yr}$ with the current HIRES precision.

We thank Karl Stapelfeldt and Sara Seager for instigating us and the JPL Program Office to make this study happen. Thanks to Steve Unwin for cheerfully and skillfully shepherding our work on this study and to Margaret Turnbull for providing target lists and associated stellar properties and for helpful conversations. We thank the many observers who contributed to the measurements reported here, including Geoff Marcy, Debra Fischer, Jason Wright, John Johnson, R. Paul Butler, and Steve Vogt. We gratefully acknowledge the efforts and dedication of the staffs of Lick Observatory and Keck Observatory and the time assignment committees of the University of Hawaii, the University of California, NASA, 
the California Institute of Technology, and Yale University for their generous allocations of observing time over the past two decades that enabled these measurements. We thank Geoff Marcy for helpful comments. We acknowledge NASA-JPL award \#1505707. This work made use of the SIMBAD database (operated at CDS, Strasbourg, France), NASA's Astrophysics Data System Bibliographic Services, the NASA Star and Exoplanet Database (NStED), the Exoplanet Orbit Database and the Exoplanet Data Explorer at exoplanets.org. Finally, we extend special thanks to those of Hawai'ian ancestry on whose sacred mountain of Maunakea

we are privileged to be guests. Without their generous hospitality, the Keck observations presented herein would not have been possible.

Facilities: Lick (Hamilton Spectrograph), Keck (HIRES)

\section{REFERENCES}

Backman, D., et al. 2009, ApJ, 690, 1522

Bottom, M., Muirhead, P. S., Johnson, J. A., \& Blake, C. H. 2013, PASP, 125, 240

Bouchy, F., Pepe, F., \& Queloz, D. 2001, A\&A, 374, 733

Butler, R. P., Marcy, G. W., Fischer, D. A., Brown, T. M., Contos, A. R., Korzennik, S. G., Nisenson, P., \& Noyes, R. W. 1999, ApJ, 526, 916

Butler, R. P., Marcy, G. W., Williams, E., Hauser, H., \& Shirts, P. 1997, ApJ, 474, L115

Butler, R. P., Marcy, G. W., Williams, E., McCarthy, C., Dosanjh, P., \& Vogt, S. S. 1996, PASP, 108, 500

Carter, J. A., et al. 2012, Science, 337, 556

Crepp, J. R., Johnson, J. A., Howard, A. W., Marcy, G. W., Brewer, J., Fischer, D. A., Wright, J. T., \& Isaacson, H. 2014, ApJ, 781, 29

Crepp, J. R., Johnson, J. A., Howard, A. W., Marcy, G. W., Gianninas, A., Kilic, M., \& Wright, J. T. 2013a, ApJ, 774, 1

Crepp, J. R., et al. 2012a, ApJ, 751, 97

—. 2012b, ApJ, 761, 39

—. 2013b, ApJ, 771, 46 
Crockett, C. J., Mahmud, N. I., Prato, L., Johns-Krull, C. M., Jaffe, D. T., Hartigan, P. M., \& Beichman, C. A. 2012, ApJ, 761, 164

Cumming, A., Butler, R. P., Marcy, G. W., Vogt, S. S., Wright, J. T., \& Fischer, D. A. 2008, PASP, 120, 531

Cumming, A., Marcy, G. W., \& Butler, R. P. 1999, ApJ, 526, 890

Dawson, R. I., \& Fabrycky, D. C. 2010, ApJ, 722, 937

Desort, M., et al. 2009, A\&A, 506, 1469

Drake, F. D. 1961, Physics Today, 14, 40

Dumusque, X., Santos, N. C., Udry, S., Lovis, C., \& Bonfils, X. 2011a, A\&A, 527, A82

Dumusque, X., Udry, S., Lovis, C., Santos, N. C., \& Monteiro, M. J. P. F. G. 2011b, A\&A, 525, A140

Dumusque, X., et al. 2011c, A\&A, 535, A55

Fischer, D., et al. 2009, ApJ, 703, 1545

Fischer, D. A., Butler, R. P., Marcy, G. W., Vogt, S. S., \& Henry, G. W. 2003, ApJ, 590, 1081

Fischer, D. A., Marcy, G. W., Butler, R. P., Laughlin, G., \& Vogt, S. S. 2002, ApJ, 564, 1028

Fischer, D. A., Marcy, G. W., \& Spronck, J. F. P. 2014, ApJS, 210, 5

Fischer, D. A., et al. 2005, ApJ, 620, 481

Galland, F., Lagrange, A.-M., Udry, S., Chelli, A., Pepe, F., Queloz, D., Beuzit, J.-L., \& Mayor, M. 2005, A\&A, 443, 337

Gliese, W. 1969, Veroeffentlichungen des Astronomischen Rechen-Instituts Heidelberg, 22, 1

Gliese, W., \& Jahreiß, H. 1979, A\&AS, 38, 423

Golimowski, D. A., Fastie, W. G., Schroeder, D. J., \& Uomoto, A. 1995a, ApJ, 452, L125

Golimowski, D. A., Nakajima, T., Kulkarni, S. R., \& Oppenheimer, B. R. 1995b, ApJ, 444, L101 
Goullioud, R., Zhao, F., Tang, H., \& Wu, J. 2014, in Society of Photo-Optical Instrumentation Engineers (SPIE) Conference Series, Vol. 9143, Society of Photo-Optical Instrumentation Engineers (SPIE) Conference Series

Greaves, J. S., et al. 1998, ApJ, 506, L133

Gregory, P. C., \& Fischer, D. A. 2010, MNRAS, 403, 731

Grunblatt, S. K., Howard, A. W., \& Haywood, R. D. 2015, ApJ, 808, 127

Hatzes, A. P., et al. 2000, ApJ, 544, L145

Haywood, R. D., et al. 2014, MNRAS, 443, 2517

Hébrard, É. M., Donati, J.-F., Delfosse, X., Morin, J., Boisse, I., Moutou, C., \& Hébrard, G. 2014, MNRAS, 443, 2599

Hillenbrand, L., Isaacson, H., Marcy, G., Barenfeld, S., Fischer, D., \& Howard, A. 2014, arXiv:1408.3475

Hoffleit, D., \& Jaschek, C. 1982, The Bright Star Catalogue (Yale Univ. Obs.)

Horne, J. H., \& Baliunas, S. L. 1986, ApJ, 302, 757

Howard, A. W., et al. 2009, ApJ, 696, 75

—. 2010a, ApJ, 721, 1467

—. 2010b, Science, 330, 653

-. 2011a, ApJ, 726, 73

-. 2011b, ApJ, 730, 10

-. 2014, ApJ, 794, 51

Isaacson, H., \& Fischer, D. 2010, ApJ, 725, 875

Jeffers, S. V., Petit, P., Marsden, S. C., Morin, J., Donati, J.-F., \& Folsom, C. P. 2014, A\&A, 569, A79

Johnson, J. A., et al. 2007, ApJ, 665, 785

Kjeldsen, H., \& Bedding, T. R. 1995, A\&A, 293, 87

Knutson, H. A., et al. 2014, ApJ, 785, 126 
Konacki, M., Muterspaugh, M. W., Kulkarni, S. R., \& Hełminiak, K. G. 2009, ApJ, 704, 513

Liu, M. C., Leggett, S. K., \& Chiu, K. 2007, ApJ, 660, 1507

Lomb, N. R. 1976, Ap\&SS, 39, 447

López-Santiago, J., Montes, D., Crespo-Chacón, I., \& Fernández-Figueroa, M. J. 2006, ApJ, 643,1160

Lovis, C., et al. 2006, Nature, 441, 305

—. 2011, arXiv:1107.5325

Luhman, K. L., et al. 2007, ApJ, 654, 570

Maire, A.-L., et al. 2014, A\&A, 566, A126

Mamajek, E. E., \& Hillenbrand, L. A. 2008, ApJ, 687, 1264

Maness, H. L., Marcy, G. W., Ford, E. B., Hauschildt, P. H., Shreve, A. T., Basri, G. B., Butler, R. P., \& Vogt, S. S. 2007, PASP, 119, 90

Marcy, G., Butler, R. P., Fischer, D., Vogt, S., Wright, J. T., Tinney, C. G., \& Jones, H. R. A. 2005a, Progress of Theoretical Physics Supplement, 158, 24

Marcy, G. W., \& Butler, R. P. 1992, PASP, 104, 270

-. 1996, ApJ, 464, L147

-. 2000, PASP, 112, 137

Marcy, G. W., Butler, R. P., \& Vogt, S. S. 2000, ApJ, 536, L43

Marcy, G. W., Butler, R. P., Vogt, S. S., Fischer, D. A., Henry, G. W., Laughlin, G., Wright, J. T., \& Johnson, J. A. 2005b, ApJ, 619, 570

Marcy, G. W., Butler, R. P., Williams, E., Bildsten, L., Graham, J. R., Ghez, A. M., \& Jernigan, J. G. 1997, ApJ, 481, 926

Marcy, G. W., et al. 2014, ApJS, 210, 20

Marengo, M., Stapelfeldt, K., Werner, M. W., Hora, J. L., Fazio, G. G., Schuster, M. T., Carson, J. C., \& Megeath, S. T. 2009, ApJ, 700, 1647

Mayor, M., \& Queloz, D. 1995, Nature, 378, 355 
Mayor, M., Udry, S., Naef, D., Pepe, F., Queloz, D., Santos, N. C., \& Burnet, M. 2004, A\&A, 415, 391

Mayor, M., et al. 2011, arXiv:1109.2497

Metcalfe, T. S., et al. 2013, ApJ, 763, L26

Montet, B. T., Crepp, J. R., Johnson, J. A., Howard, A. W., \& Marcy, G. W. 2014, ApJ, 781,28

Moran, S. M., Kuchner, M. J., \& Holman, M. J. 2004, ApJ, 612, 1163

Mugrauer, M., Seifahrt, A., Neuhäuser, R., \& Mazeh, T. 2006, MNRAS, 373, L31

Murray, C., \& Dermott, S. 1999, Solar System Dynamics (Cambridge University Press)

O’Toole, S. J., Tinney, C. G., Jones, H. R. A., Butler, R. P., Marcy, G. W., Carter, B., \& Bailey, J. 2009, MNRAS, 392, 641

Pasquini, L., Cristiani, S., Garcia-Lopez, R., Haehnelt, M., \& Mayor, M. 2010, The Messenger, 140, 20

Pepe, F., et al. 2011, A\&A, 534, A58

—. 2014, Astronomische Nachrichten, 335, 8

Pepe, F. A., \& Lovis, C. 2008, Physica Scripta Volume T, 130, 014007

Quirrenbach, A., Reffert, S., \& Bergmann, C. 2011, in American Institute of Physics Conference Series, Vol. 1331, American Institute of Physics Conference Series, ed. S. Schuh, H. Drechsel, \& U. Heber, 102-109

Reffert, S., Quirrenbach, A., Mitchell, D. S., Albrecht, S., Hekker, S., Fischer, D. A., Marcy, G. W., \& Butler, R. P. 2006, ApJ, 652, 661

Rivera, E. J., Laughlin, G., Butler, R. P., Vogt, S. S., Haghighipour, N., \& Meschiari, S. 2010, ApJ, 719, 890

Robinson, S. E., Ammons, S. M., Kretke, K. A., Strader, J., Wertheimer, J. G., Fischer, D. A., \& Laughlin, G. 2007, ApJS, 169, 430

Scargle, J. D. 1982, ApJ, 263, 835

Schnupp, C., et al. 2010, A\&A, 516, A21 
Seager, S., et al. 2014, in American Astronomical Society Meeting Abstracts, Vol. 224, American Astronomical Society Meeting Abstracts \#224, \#311.06

Spergel, D., et al. 2013, ArXiv e-prints

Stapelfeldt, K. R., et al. 2014, in Society of Photo-Optical Instrumentation Engineers (SPIE) Conference Series, Vol. 9143, Society of Photo-Optical Instrumentation Engineers (SPIE) Conference Series

Strand, K. A. 1943, PASP, 55, 29

-. 1957, AJ, 62, 35

Tan, X., Payne, M. J., Lee, M. H., Ford, E. B., Howard, A. W., Johnson, J. A., Marcy, G. W., \& Wright, J. T. 2013, ApJ, 777, 101

Tanner, A. M., Gelino, C. R., \& Law, N. M. 2010, PASP, 122, 1195

Tinney, C. G., Butler, R. P., Marcy, G. W., Jones, H. R. A., Penny, A. J., Vogt, S. S., Apps, K., \& Henry, G. W. 2001, ApJ, 551, 507

Traub, W. A., et al. 2014, in Society of Photo-Optical Instrumentation Engineers (SPIE) Conference Series, Vol. 9143, Society of Photo-Optical Instrumentation Engineers (SPIE) Conference Series

Trifonov, T., Reffert, S., Tan, X., Lee, M. H., \& Quirrenbach, A. 2014, A\&A, 568, A64

Tuomi, M., et al. 2013, A\&A, 551, A79

Valenti, J. A., Butler, R. P., \& Marcy, G. W. 1995, PASP, 107, 966

Valenti, J. A., \& Fischer, D. A. 2005, ApJS, 159, 141

Vogt, S. S. 1987, PASP, 99, 1214

Vogt, S. S., et al. 1994, in Proc. SPIE Instrumentation in Astronomy VIII, David L. Crawford; Eric R. Craine; Eds., Vol. 2198, p. 362

Vogt, S. S., et al. 2010, ApJ, 708, 1366

Walker, G. A. H., Walker, A. R., Irwin, A. W., Larson, A. M., Yang, S. L. S., \& Richardson, D. C. 1995, Icarus, 116, 359

Winn, J. N., et al. 2011, ApJ, 737, L18 
Wittenmyer, R. A., Endl, M., Cochran, W. D., Hatzes, A. P., Walker, G. A. H., Yang, S. L. S., \& Paulson, D. B. 2006, AJ, 132, 177

Wright, J. T., \& Howard, A. W. 2009, ApJS, 182, 205

Wright, J. T., Marcy, G. W., Butler, R. P., \& Vogt, S. S. 2004, ApJS, 152, 261

Wright, J. T., Marcy, G. W., Butler, R. P., Vogt, S. S., Henry, G. W., Isaacson, H., \& Howard, A. W. 2008, ApJ, 683, L63

Wright, J. T., Upadhyay, S., Marcy, G. W., Fischer, D. A., Ford, E. B., \& Johnson, J. A. 2009, ApJ, 693, 1084

Wright, J. T., et al. 2011, PASP, 123, 412 

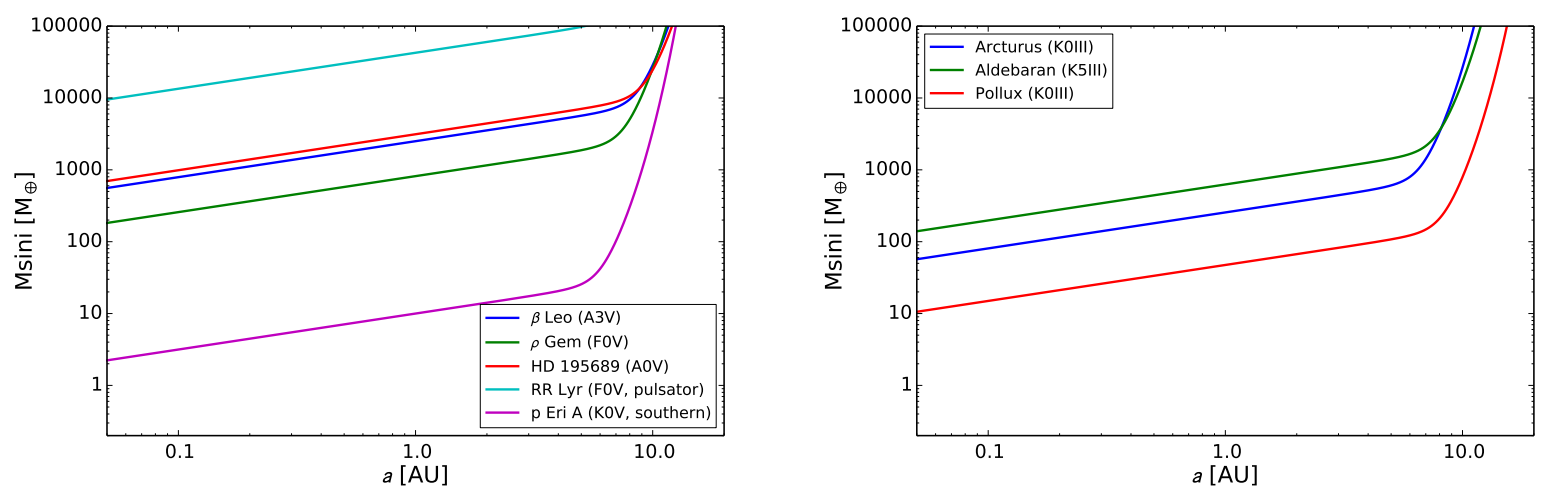

Fig. 11. - Simulated planet sensitivity thresholds, $M_{p} \sin i_{50}(P)$, for a representative sample of early-type and Southern Sun-like stars that were not observed by the Lick and Keck planet searches (left) and evolved stars (right). See Sec. 5.6 for details on the selected stars and the method to compute the sensitivity thresholds. We adopt $\alpha=6$ for these plots. These predictions depend critically on the estimated RV scatter, $\sigma_{\mathrm{RV}}$, which is uncertain at the factor of two or more level for most stars. The excellent sensitivity for p Eri A $\left(\sim 10 M_{\oplus}\right.$ at $1 \mathrm{AU}$ ) is driven by our adopted $\sigma_{\mathrm{RV}}=1 \mathrm{~m} \mathrm{~s}^{-1}$, a factor of 2-2.5 better than we routinely achieve with HIRES. See also the caveats to the idealized completeness method in Sec. 5.5. 

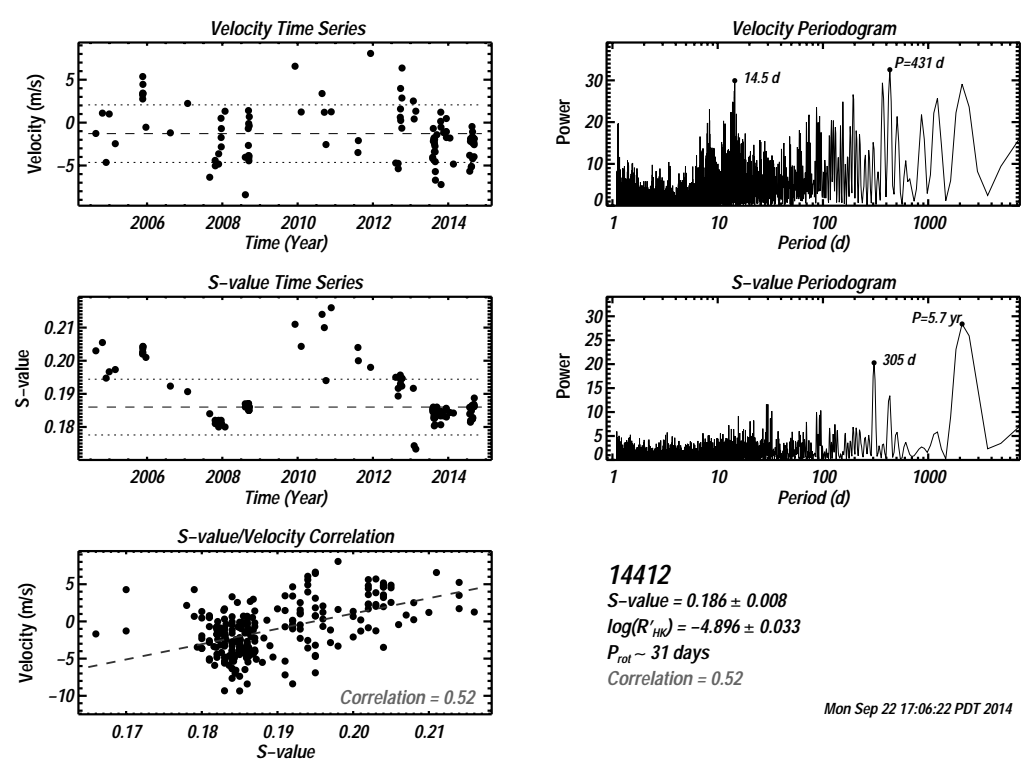

\section{2}

$S$-value $=0.186 \pm 0.008$ $\log \left(R_{H K}^{\prime}\right)=-4.896 \pm 0.033$

$P_{\text {rot }} \sim 31$ days

Correlation $=0.52$

Mon Sep 22 17:06:22 PDT 2014
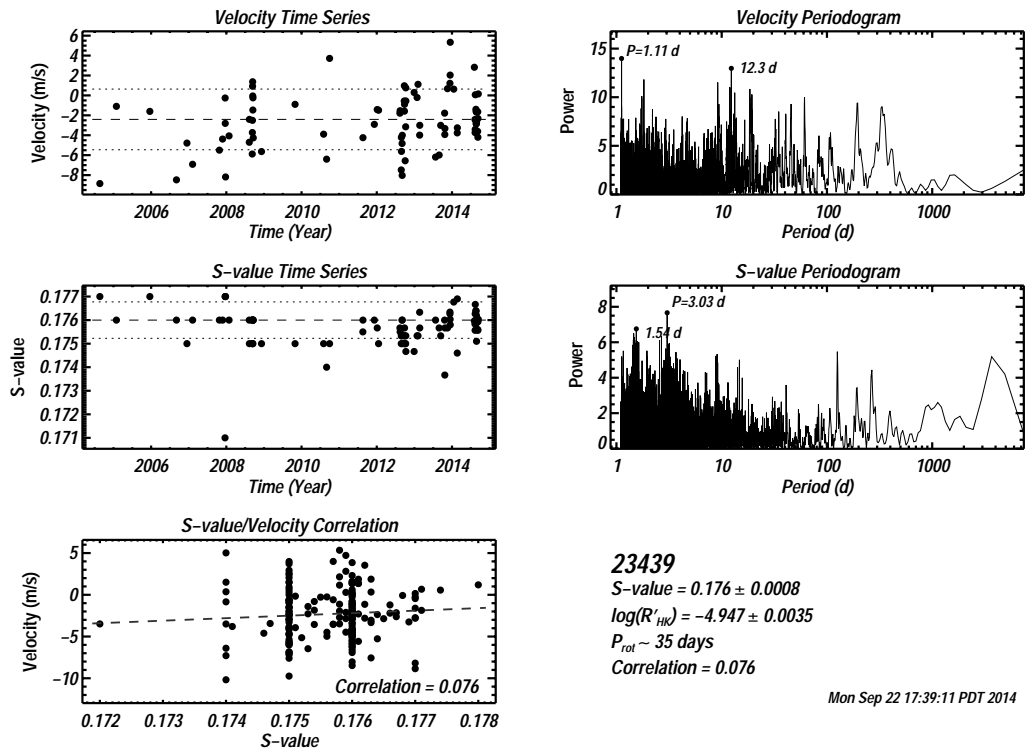

23439

$S$-value $=0.176 \pm 0.0008$ $\log \left(R^{\prime}{ }_{\text {нसk }}\right)=-4.947 \pm 0.0035$

$P_{\text {rot }} \sim 35$ days

Correlation $=0.076$

Mon Sep 22 17:39:11 PDT 2014

Fig. 12.- Automated reports on the correlations between RVs and $S_{\mathrm{HK}}$ activity measurements for two stars observed by the California Planet Survey using Keck-HIRES. Each six-panel plot (HD 14412 - top; HD 23439 - bottom) shows the RV time series (top left) and its periodogram (top right), the $S_{\mathrm{HK}}$ time series (middle left) and its periodogram (middle right), a linear correlation between them (lower left), and annotations (lower right). Both stars are considered "inactive," yet HD 14412 displays a clear RV- $S_{\mathrm{HK}}$ correlation resulting in $\sim 3 \mathrm{~m} \mathrm{~s}^{-1}$ jitter with a timescale of $5.7 \mathrm{yr}$ (presumably the stellar rotation period). HD 23429 is less active $\left(\log R_{\mathrm{HK}}^{\prime}=-4.95\right.$ vs. -4.90$)$ and has no detectable RV- $S_{\mathrm{HK}}$ correlation. 

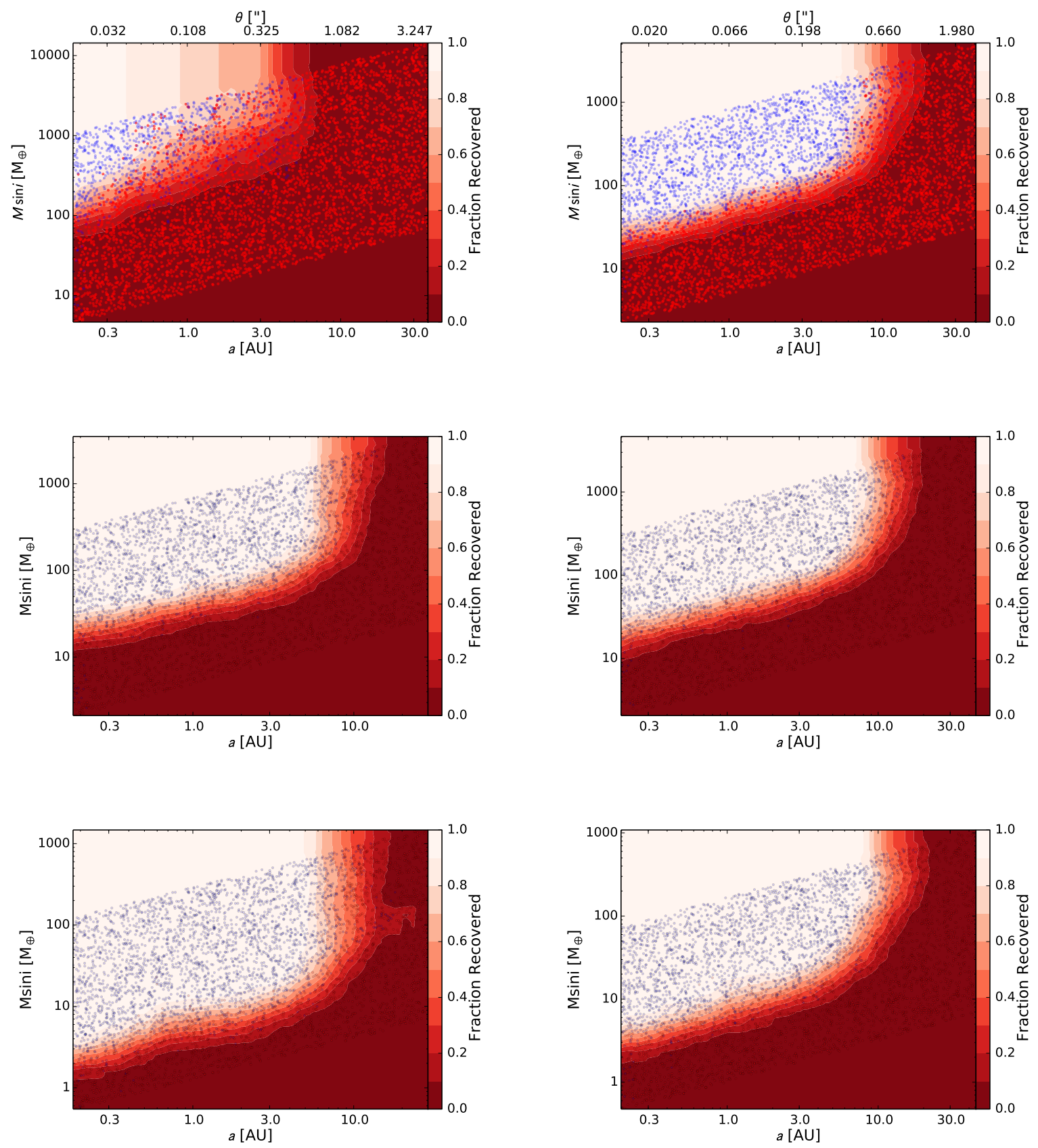

Fig. 13. - Completeness for two stars, HD 102365 (left) and HD 182572 (right). The top row shows the completeness with the observations to date (through 2014). The middle row shows the expected completeness of the same stars after adding ten years of measurements with 3 RVs per year having the same precision as the recent Keck-HIRES observations. The bottom row shows the expected completeness after adding 10 years of $10 \mathrm{RVs}$ per year with $\sigma_{\mathrm{RV}}=$ $0.5 \mathrm{~m} \mathrm{~s}^{-1}$. Such a high-precision planet search would be sensitive to $\lesssim 10 M_{\oplus}$ super-Earth planets in $3 \mathrm{AU}$ orbits. 


\section{A. Machine-readable Data}

The set of RV time series for every star with measurements is supplied as a supplement to this report. ${ }^{2}$ Each star has a separate ASCII file for RV data that is named starname_rv.cSV (e.g., 10700_rv.csv). The file header lists the star name and instrument codes. Each column is briefly labeled in the first row. The four data columns are Heliocentric Julian Date (HJD) less 2,440,000, relative radial velocity $\left(\mathrm{m} \mathrm{s}^{-1}\right)$, uncertainty in the radial velocity $\left(\mathrm{m} \mathrm{s}^{-1}\right)$, and instrument code. Statistical uncertainties in determining the Doppler shifts are accounted for, but astrophysical jitter is not.

We summarize the completeness contours for each star (Appendix C) with two sets of machine-readable files using a format similar to the RV files. The first set of ASCII files are named starname_contours.csv (e.g., 10700_contours.csv). The header lists the star name as well as the stellar mass and distance used to convert between orbital period, semi-major axis, and projected sky separation. Each column is briefly labeled in the first row. The six data columns are orbital period (days), semi-major axis (AU), sky-projected angular distance (arcsec), and the $M \sin i\left(M_{\oplus}\right)$ values that corresponds to completeness levels of $16 \%, 50 \%$, and $84 \%$, respectively.

A second set of ASCII files provide complete, two-dimensional sampling of the completeness measurements. In files named starname_2D_recovery.txt (e.g., 10700_2D_recovery.txt), we list the completeness values (the red shading) at every cell in the completeness plots. These files have headers listing the star masses, distances and column names. The columns are sky-projected angular distance (arcsec), and the $M \sin i\left(M_{\oplus}\right)$, and fractional completeness $(0-1)$.

\section{B. Properties of Stars without Doppler Measurements}

Table B.1 contains the complete list of 244 stars that are on the Exo-S and Exo-C target lists and were not observed at Lick or Keck Observatories. The Notes column lists the reason(s) that the stars were likely not included in our Keck/Lick Planet Searches. This

table is the complement to Table 1, which includes stars with observations. Because of the quantity of this data, we relegated Table B.1 to this appendix.

\footnotetext{
${ }^{2}$ All machine-readable data products can be downloaded from http://exoplanetarchive.ipac.caltech.edu/docs/contributed_data.html
} 
Table B.1. Imaging Target Stars Without Doppler Measurements

\begin{tabular}{|c|c|c|c|c|c|c|c|c|c|}
\hline $\begin{array}{l}\text { Hipp. } \\
\text { No. }\end{array}$ & $\begin{array}{l}\text { HD } \\
\text { No. }\end{array}$ & $\begin{array}{l}\text { Gliese } \\
\text { No. }\end{array}$ & $\begin{array}{c}\text { Target }^{\mathrm{a}} \\
\text { List }\end{array}$ & $\begin{array}{l}\text { Dist. } \\
\text { (pc) }\end{array}$ & $\begin{array}{c}V \\
(\mathrm{mag})\end{array}$ & $\begin{array}{c}B-V \\
(\mathrm{mag})\end{array}$ & $\begin{array}{l}T_{\text {eff }} \\
(\mathrm{K})\end{array}$ & Sp. T. & Notes $^{\mathrm{b}}$ \\
\hline 171 & 224930 & $914 \mathrm{~A}$ & $\mathrm{~S}$ & 12.170 & 5.800 & 0.690 & 5502 & G3V & B \\
\hline 677 & 358 & $\cdots$ & $\mathrm{C}, \mathrm{A}$ & 29.740 & 2.060 & -0.048 & 13098 & B9p & $\mathrm{H}$ \\
\hline 746 & 432 & 8 & $\mathrm{C}, \mathrm{A}$ & 16.780 & 2.260 & 0.365 & 6915 & F2III-IV & $\mathrm{H}$ \\
\hline 910 & 693 & 10 & $\mathrm{C}, \mathrm{A}$ & 18.750 & 4.890 & 0.487 & 6255 & F5V & $\mathrm{H}$ \\
\hline 950 & 739 & 3013 & $\mathrm{~A}$ & 21.280 & 5.240 & 0.430 & 6548 & $\mathrm{~F} 5 \mathrm{~V}$ & $\mathrm{H}, \mathrm{S}$ \\
\hline 1599 & 1581 & 17 & $\mathrm{~S}, \mathrm{C}, \mathrm{A}$ & 8.590 & 4.230 & 0.576 & 5948 & G0V & $\mathrm{S}$ \\
\hline 2021 & 2151 & 19 & $\mathrm{~S}, \mathrm{C}, \mathrm{A}$ & 7.460 & 2.820 & 0.618 & 5873 & G1IV & $\mathrm{S}$ \\
\hline 2072 & 2262 & 20 & $\mathrm{C}, \mathrm{A}$ & 23.810 & 3.930 & 0.175 & 7922 & $\mathrm{~A} 6 \mathrm{VN}$ & $\mathrm{S}$ \\
\hline 2081 & 2261 & $\ldots$ & $\mathrm{C}$ & 25.970 & 2.400 & 1.083 & 4436 & K0IIIB & $\mathrm{E}$ \\
\hline 2762 & 3196 & $23 \mathrm{~A}$ & $\mathrm{~A}$ & 21.250 & 5.200 & 0.570 & 6067 & F8V & B \\
\hline 2941 & 3443 & $25 \mathrm{~A}$ & $\mathrm{~S}$ & 15.400 & 5.570 & 0.715 & 5480 & $\mathrm{~K} 1 \mathrm{~V}+\mathrm{G}$ & B \\
\hline 3419 & 4128 & 31 & $\mathrm{C}$ & 29.530 & 2.020 & 1.038 & 4944 & KOIII & $\mathrm{E}$ \\
\hline 3505 & 4247 & 31.3 & $\mathrm{~A}$ & 26.740 & 5.220 & 0.320 & 6903 & F3V & $\mathrm{H}$ \\
\hline 3810 & 4676 & 34.1 & $\mathrm{~A}$ & 23.450 & 5.070 & 0.500 & 6254 & $\mathrm{~F} 8 \mathrm{~V}$ & $\mathrm{H}$ \\
\hline 3909 & 4813 & 37 & $\mathrm{~S}, \mathrm{~A}$ & 15.753 & 5.164 & 0.514 & 6250 & F7V & $\mathrm{H}, N_{\mathrm{obs}}=2$ \\
\hline 4151 & 5015 & 41 & $\mathrm{C}, \mathrm{A}$ & 18.740 & 4.800 & 0.540 & 6196 & F8V & $\mathrm{B}$ \\
\hline 5336 & 6582 & $53 \mathrm{~A}$ & $\mathrm{~S}, \mathrm{C}, \mathrm{A}$ & 7.550 & 5.170 & 0.704 & 5526 & $\mathrm{G} 5 \mathrm{Vp}$ & B \\
\hline 5799 & 7439 & $54.2 \mathrm{~A}$ & A & 23.380 & 5.140 & 0.450 & 6465 & F5V & $\mathrm{H}$ \\
\hline 5862 & 7570 & 55 & $\mathrm{~S}, \mathrm{C}, \mathrm{A}$ & 15.110 & 4.960 & 0.571 & 6116 & G0V & $\mathrm{S}$ \\
\hline 5896 & 7788 & $55.3 \mathrm{~A}$ & $\mathrm{~A}$ & 20.950 & 4.250 & 0.450 & 6505 & F5V & $\mathrm{H}, \mathrm{S}$ \\
\hline 6537 & 8512 & $\ldots$ & $\mathrm{C}$ & 34.900 & 3.800 & 1.070 & $\ldots$ & K0III & $\mathrm{E}$ \\
\hline 6686 & 8538 & $\ldots$ & $\mathrm{C}$ & 30.500 & 2.700 & 0.160 & $\cdots$ & $\mathrm{A} 5 \mathrm{Vv}$ & $\mathrm{B}, \mathrm{H}$ \\
\hline 6706 & 8723 & $\ldots$ & $\mathrm{A}$ & 25.210 & 5.350 & 0.370 & 6690 & $\mathrm{~F} 2 \mathrm{~V}$ & $\mathrm{H}$ \\
\hline 6813 & 8799 & $\ldots$ & $\mathrm{A}$ & 28.620 & 4.830 & 0.420 & 6555 & F4V & $\mathrm{H}$ \\
\hline 7751 & 10360 & $66 \mathrm{AB}$ & $\mathrm{S}$ & 7.820 & 5.680 & 0.864 & 5016 & $\mathrm{~K} 0 / 4$ & $\mathrm{~S}$ \\
\hline 7918 & 10307 & 67 & $\mathrm{~S}, \mathrm{C}, \mathrm{A}$ & 12.740 & 4.960 & 0.618 & 5874 & $\mathrm{G} 2 \mathrm{~V}$ & B \\
\hline 8209 & 10830 & $\ldots$ & A & 28.110 & 5.290 & 0.400 & 6740 & $\mathrm{~F} 2 \mathrm{~V}$ & $\mathrm{H}$ \\
\hline 8497 & 11171 & $\ldots$ & $\mathrm{C}, \mathrm{A}$ & 23.190 & 4.650 & 0.333 & 7087 & F0V & $\mathrm{H}$ \\
\hline 8796 & 11443 & 78.1 & $\mathrm{C}, \mathrm{A}$ & 19.420 & 3.420 & 0.488 & 6273 & F6IV & B \\
\hline 8903 & 11636 & 80 & $\mathrm{C}, \mathrm{A}$ & 17.990 & 2.630 & 0.156 & 8300 & $\mathrm{~A} 5 \mathrm{~V}$ & $\mathrm{H}$ \\
\hline 9007 & 11937 & $81 \mathrm{~A}$ & $\mathrm{C}$ & 17.850 & 3.690 & 0.844 & 5182 & G8III & $\mathrm{S}, \mathrm{E}$ \\
\hline 9236 & 12311 & 83 & $\mathrm{C}, \mathrm{A}$ & 22.010 & 2.860 & 0.290 & 7201 & F0V & $\mathrm{S}, \mathrm{H}$ \\
\hline 9884 & 12929 & 84.3 & $\mathrm{C}$ & 20.180 & 2.010 & 1.151 & 4504 & K2III & $\mathrm{E}$ \\
\hline 10138 & 13445 & 86 & $\mathrm{~S}$ & 10.780 & 6.120 & 0.812 & 5151 & $\mathrm{~K} 1 \mathrm{~V}$ & $\mathrm{~S}$ \\
\hline 10306 & 13555 & $\ldots$ & $\mathrm{A}$ & 28.870 & 5.230 & 0.440 & 6496 & F5V & $\mathrm{H}$ \\
\hline 10644 & 13974 & 92 & $\mathrm{~S}, \mathrm{C}, \mathrm{A}$ & 10.780 & 4.860 & 0.607 & 5667 & G0V & $\mathrm{B}$ \\
\hline
\end{tabular}


Table B.1—Continued

\begin{tabular}{|c|c|c|c|c|c|c|c|c|c|}
\hline $\begin{array}{l}\text { Hipp. } \\
\text { No. }\end{array}$ & $\begin{array}{l}\text { HD } \\
\text { No. }\end{array}$ & $\begin{array}{c}\text { Gliese } \\
\text { No. }\end{array}$ & $\begin{array}{c}\text { Target }^{\mathrm{a}} \\
\text { List }\end{array}$ & $\begin{array}{c}\text { Dist. } \\
(\mathrm{pc})\end{array}$ & $\begin{array}{c}V \\
(\mathrm{mag})\end{array}$ & $\begin{array}{c}B-V \\
(\mathrm{mag})\end{array}$ & $\begin{array}{l}T_{\text {eff }} \\
(\mathrm{K})\end{array}$ & Sp. T. & Notes $^{b}$ \\
\hline 10670 & 14055 & $\ldots$ & $\mathrm{C}$ & 34.400 & 4.000 & 0.020 & $\ldots$ & A1Vnn & $\mathrm{H}$ \\
\hline 11072 & 14802 & 97 & $\mathrm{~A}$ & 21.950 & 5.190 & 0.610 & 5854 & G1V & B \\
\hline 11783 & 15798 & $\cdots$ & A & 26.690 & 4.740 & 0.450 & 6502 & F5V & $\mathrm{H}$ \\
\hline 12390 & 16620 & $105.4 \mathrm{~A}$ & $\mathrm{~A}$ & 26.960 & 4.830 & 0.430 & 6516 & $\mathrm{~F} 4 \mathrm{~V}$ & $\mathrm{H}$ \\
\hline 12623 & 16739 & 105.6 & $\mathrm{~A}$ & 24.190 & 4.910 & 0.580 & 5973 & F9V & $\mathrm{H}$ \\
\hline 12706 & 16970 & $106.1 \mathrm{~A}$ & $\mathrm{C}, \mathrm{A}$ & 24.410 & 3.470 & 0.093 & 8673 & $\mathrm{~A} 2 \mathrm{Va}$ & $\mathrm{H}$ \\
\hline 12777 & 16895 & $107 \mathrm{~A}$ & $\mathrm{~S}, \mathrm{C}, \mathrm{A}$ & 11.127 & 4.098 & 0.489 & 6344 & F7V & $\mathrm{H}, N_{\mathrm{obs}}=2$ \\
\hline 12828 & 17094 & $\cdots$ & $\mathrm{C}, \mathrm{A}$ & 25.770 & 4.270 & 0.311 & 7225 & F1III-IV & $\mathrm{H}$ \\
\hline 12843 & 17206 & 111 & $\mathrm{~S}, \mathrm{C}, \mathrm{A}$ & 14.220 & 4.470 & 0.481 & 6378 & $\mathrm{~F} 5 / 6 \mathrm{~V}$ & $\mathrm{H}$ \\
\hline 14146 & 18978 & 121 & $\mathrm{C}, \mathrm{A}$ & 27.170 & 4.080 & 0.163 & 8045 & $\mathrm{~A} 4 \mathrm{~V}$ & $\mathrm{H}$ \\
\hline 14576 & 19356 & $\ldots$ & $\mathrm{C}, \mathrm{A}$ & 27.570 & 2.110 & -0.010 & 9634 & B8V & $\mathrm{H}$ \\
\hline 14668 & 19476 & $\ldots$ & $\mathrm{C}$ & 34.600 & 4.000 & 0.980 & $\ldots$ & K0III & $\mathrm{E}$ \\
\hline 14879 & 20010 & $127 \mathrm{~A}$ & $\mathrm{~S}, \mathrm{C}, \mathrm{A}$ & 14.240 & 3.800 & 0.543 & 6258 & F8V & B \\
\hline 15330 & 20766 & 136 & $\mathrm{~S}$ & 12.010 & 5.530 & 0.641 & 5699 & $\mathrm{G} 3 / 5 \mathrm{~V}$ & $\mathrm{~S}$ \\
\hline 15371 & 20807 & 138 & $\mathrm{~S}, \mathrm{C}, \mathrm{A}$ & 12.030 & 5.240 & 0.600 & 5845 & $\mathrm{G} 2 \mathrm{~V}$ & $\mathrm{~S}$ \\
\hline 15510 & 20794 & 139 & $\mathrm{~S}, \mathrm{C}, \mathrm{A}$ & 6.040 & 4.260 & 0.711 & 5401 & G8.0V & $\mathrm{S}$ \\
\hline 16245 & 22001 & $143.2 \mathrm{~A}$ & $\mathrm{C}, \mathrm{A}$ & 21.680 & 4.700 & 0.410 & 6629 & F3IV/V & $\mathrm{S}, \mathrm{H}$ \\
\hline 17440 & $\ldots$ & $\cdots$ & $\mathrm{C}$ & 29.860 & 3.840 & 1.133 & 4514 & K0IV & $\mathrm{B}, \mathrm{S}$ \\
\hline 17651 & 23754 & 155 & $\mathrm{C}, \mathrm{A}$ & 17.630 & 4.220 & 0.434 & 6631 & $\mathrm{~F} 3 / 5 \mathrm{~V}$ & $\mathrm{H}$ \\
\hline 18907 & 25490 & $\cdots$ & $\mathrm{C}$ & 35.900 & 3.900 & 0.030 & $\ldots$ & A1V & $\mathrm{H}$ \\
\hline 19205 & 25867 & $\cdots$ & A & 27.600 & 5.210 & 0.340 & 6979 & F1V & $\mathrm{H}$ \\
\hline 19747 & 26967 & $\cdots$ & $\mathrm{C}$ & 35.300 & 4.000 & 1.090 & $\ldots$ & K1III & $\mathrm{E}$ \\
\hline 19893 & 27290 & 167.1 & $\mathrm{C}, \mathrm{A}$ & 20.460 & 4.250 & 0.312 & 7060 & F0V & $\mathrm{S}, \mathrm{H}$ \\
\hline 19921 & 27442 & 167.3 & $\mathrm{C}$ & 18.240 & 4.440 & 1.078 & 4846 & $\mathrm{~K} 1 / 2 \mathrm{III}$ & $\mathrm{S}, \mathrm{E}$ \\
\hline 19990 & 27045 & $\ldots$ & $\mathrm{A}$ & 28.940 & 4.930 & 0.260 & 7384 & A3M & $\mathrm{H}$ \\
\hline 21421 & 29139 & 171.1A & $\mathrm{C}$ & 20.430 & 0.870 & 1.538 & 3889 & K5III & $\mathrm{E}$ \\
\hline 21547 & 29391 & $\ldots$ & $\mathrm{A}$ & 29.430 & 5.220 & 0.280 & 7257 & F0V & $\mathrm{H}$ \\
\hline 21770 & 29875 & $174.1 \mathrm{~A}$ & $\mathrm{C}, \mathrm{A}$ & 20.170 & 4.440 & 0.342 & 6991 & F1V & $\mathrm{H}$ \\
\hline 21861 & 29992 & 176.1 & $\mathrm{~A}$ & 28.660 & 5.040 & 0.390 & 6666 & F3IV & $\mathrm{H}, \mathrm{S}$ \\
\hline 23482 & 32743 & 187 & $\mathrm{~A}$ & 26.070 & 5.370 & 0.420 & 6624 & F5V & $\mathrm{H}, \mathrm{S}$ \\
\hline 23693 & 33262 & 189 & $\mathrm{~S}, \mathrm{C}, \mathrm{A}$ & 11.650 & 4.710 & 0.526 & 6246 & $\mathrm{~F} 6 / 7 \mathrm{~V}$ & $\mathrm{~S}, \mathrm{H}$ \\
\hline 23783 & 32537 & $187.2 \mathrm{~A}$ & $\mathrm{~A}$ & 26.290 & 4.980 & 0.320 & 7018 & $\mathrm{~F} 2 \mathrm{~V}$ & $\mathrm{H}$ \\
\hline 23875 & 33111 & $\ldots$ & $\mathrm{C}, \mathrm{A}$ & 27.400 & 2.760 & 0.150 & 8377 & A3IVn & $\mathrm{H}$ \\
\hline 23941 & 33256 & 189.2 & A & 25.460 & 5.110 & 0.430 & 6411 & $\mathrm{~F} 5.5 \mathrm{~V}$ & $\mathrm{H}$ \\
\hline 24608 & 34029 & $194 \mathrm{~A}$ & $\mathrm{C}$ & 13.120 & 0.080 & 0.795 & 5356 & M1 & B \\
\hline 25110 & 33564 & 196 & A & 20.890 & 5.080 & 0.480 & 6394 & F7V & $\mathrm{H}$ \\
\hline
\end{tabular}


Table B.1—Continued

\begin{tabular}{|c|c|c|c|c|c|c|c|c|c|}
\hline $\begin{array}{l}\text { Hipp. } \\
\text { No. }\end{array}$ & $\begin{array}{l}\text { HD } \\
\text { No. }\end{array}$ & $\begin{array}{c}\text { Gliese } \\
\text { No. }\end{array}$ & $\begin{array}{c}\text { Target }^{\mathrm{a}} \\
\text { List }\end{array}$ & $\begin{array}{c}\text { Dist. } \\
(\mathrm{pc})\end{array}$ & $\begin{array}{c}V \\
(\mathrm{mag})\end{array}$ & $\begin{array}{c}B-V \\
(\mathrm{mag})\end{array}$ & $\begin{array}{l}T_{\text {eff }} \\
(\mathrm{K})\end{array}$ & Sp. T. & Notes $^{b}$ \\
\hline 25278 & 35296 & 202 & $\mathrm{~S}, \mathrm{C}, \mathrm{A}$ & 14.390 & 5.000 & 0.523 & 6202 & F8V & $\mathrm{Y}, \mathrm{B}$ \\
\hline 27072 & 38393 & $216 \mathrm{~A}$ & $\mathrm{~S}, \mathrm{C}, \mathrm{A}$ & 8.927 & 3.590 & 0.481 & 6372 & F7V & $\mathrm{Y}, N_{\mathrm{obs}}=8$ \\
\hline 27288 & 38678 & 217.1 & $\mathrm{C}, \mathrm{A}$ & 21.610 & 3.550 & 0.104 & 8337 & $\mathrm{~A} 2$ & $\mathrm{H}$ \\
\hline 27321 & 39060 & 219 & $\mathrm{C}, \mathrm{A}$ & 19.440 & 3.850 & 0.171 & 8052 & $\mathrm{~A} 5 \mathrm{~V}$ & $\mathrm{~S}, \mathrm{H}$ \\
\hline 27628 & 39425 & $\ldots$ & $\mathrm{C}$ & 26.730 & 3.100 & 1.175 & 4545 & K1.5III & $\mathrm{E}$ \\
\hline 27654 & 39364 & $\ldots$ & $\mathrm{C}$ & 34.900 & 3.900 & 0.980 & $\ldots$ & G8III/IV & $\mathrm{E}$ \\
\hline 27890 & 40409 & 224.1 & $\mathrm{C}$ & 26.250 & 4.640 & 1.048 & 4661 & K1III/IV & $\mathrm{S}, \mathrm{E}$ \\
\hline 27913 & 39587 & $222 \mathrm{AB}$ & $\mathrm{S}, \mathrm{C}, \mathrm{A}$ & 8.663 & 4.395 & 0.594 & 5882 & G0V & B \\
\hline 28103 & 40136 & 225 & $\mathrm{~S}, \mathrm{C}, \mathrm{A}$ & 14.880 & 3.710 & 0.337 & 7069 & $\mathrm{~F} 1 \mathrm{~V}$ & $\mathrm{H}$ \\
\hline 28360 & 40183 & $\ldots$ & $\mathrm{C}, \mathrm{A}$ & 24.870 & 1.890 & 0.071 & 9024 & $\mathrm{~A} 2 \mathrm{~V}$ & $\mathrm{H}$ \\
\hline 29271 & 43834 & 231 & $\mathrm{~S}, \mathrm{C}, \mathrm{A}$ & 10.200 & 5.080 & 0.714 & 5587 & G6V & $\mathrm{S}$ \\
\hline 29800 & 43386 & 9207 & $\mathrm{~A}$ & 19.250 & 5.040 & 0.430 & 6602 & F5V & $\mathrm{H}$ \\
\hline 31592 & 47205 & 239.1 & $\mathrm{C}$ & 19.750 & 3.950 & 1.063 & 4799 & K1III & $\mathrm{E}, \mathrm{B}$ \\
\hline 32349 & 48915 & $244 \mathrm{~A}$ & $\mathrm{~S}, \mathrm{C}, \mathrm{A}$ & 2.630 & -1.440 & 0.009 & 9580 & A1.0V & $\mathrm{H}$ \\
\hline 32362 & 48737 & 242 & $\mathrm{C}, \mathrm{A}$ & 18.000 & 3.320 & 0.443 & 6455 & F5IV & $\mathrm{H}$ \\
\hline 32607 & $\ldots$ & $\ldots$ & $\mathrm{C}, \mathrm{A}$ & 29.600 & 3.230 & 0.222 & 7536 & A7IV & $\mathrm{S}, \mathrm{H}$ \\
\hline 32765 & 50223 & 249.1 & $\mathrm{~A}$ & 25.260 & 5.140 & 0.450 & 6482 & F5.5V & $\mathrm{H}, \mathrm{S}$ \\
\hline 33202 & 50635 & $\ldots$ & $\mathrm{C}, \mathrm{A}$ & 25.630 & 4.730 & 0.321 & 7064 & F0Vp & $\mathrm{H}$ \\
\hline 33302 & 51199 & $\ldots$ & A & 29.580 & 4.660 & 0.370 & 6790 & F3V & $\mathrm{H}$ \\
\hline 34065 & 53705 & $264.1 \mathrm{~A}$ & $\mathrm{~S}$ & 16.520 & 5.560 & 0.624 & 5827 & G3V & $\mathrm{B}, \mathrm{S}$ \\
\hline 34834 & 55892 & 268.1 & $\mathrm{C}, \mathrm{A}$ & 21.430 & 4.490 & 0.324 & 6907 & F0IV & $\mathrm{H}$ \\
\hline 35350 & 56537 & $\ldots$ & $\mathrm{C}$ & 30.900 & 3.600 & 0.110 & $\ldots$ & A3V & $\mathrm{H}$ \\
\hline 35550 & 56986 & $271 \mathrm{~A}$ & $\mathrm{C}, \mathrm{A}$ & 18.540 & 3.500 & 0.374 & 6906 & F0IV & $\mathrm{H}$ \\
\hline 36046 & 58207 & $\ldots$ & $\mathrm{C}$ & 36.900 & 4.000 & 1.020 & $\ldots$ & G9III & $\mathrm{E}$ \\
\hline 36366 & 58946 & $274 \mathrm{~A}$ & $\mathrm{C}, \mathrm{A}$ & 18.050 & 4.160 & 0.320 & 7035 & F0V & $\mathrm{H}$ \\
\hline 36439 & 58855 & $\ldots$ & $\mathrm{A}$ & 20.240 & 5.350 & 0.450 & 6457 & $\mathrm{~F} 6 \mathrm{~V}$ & $\mathrm{H}$ \\
\hline 36795 & 60532 & 279 & $\mathrm{C}, \mathrm{A}$ & 25.300 & 4.440 & 0.521 & 6262 & F6V & $\mathrm{H}$ \\
\hline 36850 & 60179 & $278 \mathrm{~A}$ & $\mathrm{~S}, \mathrm{C}, \mathrm{A}$ & 15.600 & 1.580 & 0.034 & 8932 & $\mathrm{~A} 2 \mathrm{Vm}$ & $\mathrm{H}$ \\
\hline 37279 & 61421 & $280 \mathrm{~A}$ & $\mathrm{~S}, \mathrm{C}, \mathrm{A}$ & 3.510 & 0.400 & 0.432 & 6543 & F5IV-V & $\mathrm{H}$ \\
\hline 37606 & 62644 & 284 & $\mathrm{~A}$ & 24.670 & 5.040 & 0.760 & 5343 & G8IV-V & $\mathrm{S}$ \\
\hline 37826 & 62509 & 286 & $\mathrm{C}$ & 10.360 & 1.160 & 0.991 & 4850 & K0IIIvar & $\mathrm{E}$ \\
\hline 37853 & 63077 & $288 \mathrm{~A}$ & $\mathrm{~S}, \mathrm{~A}$ & 15.210 & 5.360 & 0.589 & 6002 & G0V & B \\
\hline 38382 & 64096 & $291 \mathrm{~A}$ & $\mathrm{~S}, \mathrm{~A}$ & 16.500 & 5.160 & 0.600 & 5826 & $\mathrm{G} 2 \mathrm{~V}$ & B \\
\hline 38423 & 64379 & $292 \mathrm{~A}$ & A & 17.940 & 5.010 & 0.430 & 6554 & $\mathrm{~F} 5 \mathrm{~V}$ & $\mathrm{H}, \mathrm{S}, \mathrm{B}$ \\
\hline 38908 & 65907 & $294 \mathrm{~A}$ & $\mathrm{~S}$ & 16.200 & 5.580 & 0.573 & 5949 & G0V & $\mathrm{S}$ \\
\hline 39757 & 67523 & $\ldots$ & $\mathrm{C}, \mathrm{A}$ & 19.480 & 2.790 & 0.456 & 6449 & $\mathrm{~F} 2$ & $\mathrm{H}$ \\
\hline
\end{tabular}


Table B.1—Continued

\begin{tabular}{|c|c|c|c|c|c|c|c|c|c|}
\hline $\begin{array}{c}\text { Hipp. } \\
\text { No. }\end{array}$ & $\begin{array}{l}\text { HD } \\
\text { No. }\end{array}$ & $\begin{array}{c}\text { Gliese } \\
\text { No. }\end{array}$ & $\begin{array}{c}\text { Target }^{\mathrm{a}} \\
\text { List }\end{array}$ & $\begin{array}{c}\text { Dist. } \\
\text { (pc) }\end{array}$ & $\begin{array}{c}V \\
(\mathrm{mag})\end{array}$ & $\begin{array}{c}B-V \\
(\mathrm{mag})\end{array}$ & $\begin{array}{l}T_{\text {eff }} \\
(\mathrm{K})\end{array}$ & Sp. T. & Notes $^{b}$ \\
\hline 39903 & 68456 & 297.1 & $\mathrm{C}, \mathrm{A}$ & 19.980 & 4.740 & 0.437 & 6467 & F5V & $\mathrm{S}, \mathrm{H}$ \\
\hline 40167 & 68255 & $\cdots$ & $\mathrm{C}, \mathrm{A}$ & 25.080 & 5.240 & 0.531 & 5741 & G0V & B \\
\hline 40702 & 71243 & 305 & $\mathrm{C}, \mathrm{A}$ & 19.560 & 4.050 & 0.404 & 6625 & F5V & $\mathrm{S}, \mathrm{H}$ \\
\hline 40706 & 70060 & 1109 & $\mathrm{C}, \mathrm{A}$ & 28.630 & 4.440 & 0.222 & 7790 & A4 & $\mathrm{H}$ \\
\hline 41312 & 71878 & $\ldots$ & $\mathrm{C}$ & 33.000 & 3.900 & 1.130 & $\ldots$ & K2IIIvar & $\mathrm{S}, \mathrm{E}$ \\
\hline 42430 & 73752 & $314 \mathrm{~A}$ & A & 19.400 & 5.050 & 0.710 & 5499 & G5IV & $\mathrm{E}$ \\
\hline 42913 & 74956 & $321.3 \mathrm{~A}$ & $\mathrm{C}, \mathrm{A}$ & 24.700 & 1.930 & 0.043 & 9021 & A0V & $\mathrm{S}, \mathrm{H}$ \\
\hline 44127 & 76644 & $331 \mathrm{~A}$ & $\mathrm{~S}, \mathrm{C}, \mathrm{A}$ & 14.510 & 3.100 & 0.207 & 7769 & A7IV & $\mathrm{H}$ \\
\hline 44143 & 77370 & 333.1 & $\mathrm{~A}$ & 26.420 & 5.170 & 0.420 & 6690 & $\mathrm{~F} 4 \mathrm{~V}$ & $\mathrm{H}, \mathrm{S}$ \\
\hline 44248 & 76943 & $332 \mathrm{~A}$ & $\mathrm{~S}, \mathrm{C}, \mathrm{A}$ & 16.070 & 3.970 & 0.443 & 6538 & F5V & $\mathrm{H}$ \\
\hline 44901 & 78209 & $\ldots$ & $\mathrm{C}, \mathrm{A}$ & 28.820 & 4.440 & 0.288 & 7231 & $\mathrm{Am}$ & $\mathrm{H}$ \\
\hline 45038 & 78154 & $335 \mathrm{~A}$ & $\mathrm{C}, \mathrm{A}$ & 20.380 & 4.800 & 0.489 & 6180 & F7IV-V & B \\
\hline 45238 & 80007 & $\ldots$ & $\mathrm{C}$ & 34.700 & 1.700 & 0.070 & $\ldots$ & A2IV & $\mathrm{S}, \mathrm{H}$ \\
\hline 45333 & 79028 & 337.1 & A & 19.570 & 5.180 & 0.590 & 5871 & G0V & B \\
\hline 46509 & 81997 & $348 \mathrm{~A}$ & $\mathrm{C}, \mathrm{A}$ & 17.330 & 4.590 & 0.411 & 6488 & F5V & $\mathrm{H}$ \\
\hline 46651 & 82434 & $351 \mathrm{~A}$ & $\mathrm{C}, \mathrm{A}$ & 18.810 & 3.600 & 0.371 & 6837 & F2IV & $\mathrm{H}$ \\
\hline 46733 & 81937 & $\ldots$ & $\mathrm{C}, \mathrm{A}$ & 23.820 & 3.640 & 0.360 & 6875 & F0IV & $\mathrm{H}$ \\
\hline 46853 & 82328 & $354 \mathrm{~A}$ & $\mathrm{~S}, \mathrm{C}, \mathrm{A}$ & 13.480 & 3.160 & 0.469 & 6334 & F6IV & $\mathrm{H}$ \\
\hline 47080 & 82885 & $356 \mathrm{~A}$ & $\mathrm{~S}, \mathrm{C}, \mathrm{A}$ & 11.370 & 5.390 & 0.770 & 5370 & G8IV-V & B \\
\hline 48319 & 84999 & $\ldots$ & $\mathrm{C}$ & 35.600 & 3.800 & 0.290 & $\ldots$ & F0IV & $\mathrm{H}$ \\
\hline 48833 & 86146 & $\ldots$ & A & 28.130 & 5.110 & 0.470 & 6393 & $\mathrm{~F} 6 \mathrm{~V}$ & $\mathrm{H}$ \\
\hline 49593 & 87696 & 378.3 & $\mathrm{C}, \mathrm{A}$ & 28.240 & 4.490 & 0.190 & 7839 & $\mathrm{~A} 7 \mathrm{~V}$ & $\mathrm{H}$ \\
\hline 49669 & 87901 & $\ldots$ & $\mathrm{C}, \mathrm{A}$ & 24.310 & 1.410 & -0.041 & 11962 & B7V & $\mathrm{H}$ \\
\hline 49809 & 88215 & $\cdots$ & A & 27.730 & 5.300 & 0.370 & 6776 & F3V & $\mathrm{H}$ \\
\hline 50191 & 88955 & $\ldots$ & $\mathrm{C}$ & 31.100 & 3.900 & 0.050 & $\ldots$ & $\mathrm{A} 2 \mathrm{~V}$ & $\mathrm{H}$ \\
\hline 50564 & 89449 & 388.1 & $\mathrm{C}, \mathrm{A}$ & 21.370 & 4.780 & 0.452 & 6476 & F6IV & $\mathrm{E}$ \\
\hline 50954 & 90589 & 391 & $\mathrm{~S}, \mathrm{C}, \mathrm{A}$ & 16.220 & 3.980 & 0.369 & 6885 & $\mathrm{~F} 2 / 3 \mathrm{IV} / \mathrm{V}$ & $\mathrm{S}, \mathrm{H}$ \\
\hline 51502 & 90089 & 392.1 & A & 21.480 & 5.250 & 0.370 & 6762 & F4V & $\mathrm{H}$ \\
\hline 51523 & 91324 & 397.2 & $\mathrm{~A}$ & 21.810 & 4.890 & 0.500 & 6287 & F9V & $\mathrm{S}$ \\
\hline 51814 & 91480 & $\cdots$ & $\mathrm{A}$ & 26.520 & 5.160 & 0.330 & 6972 & $\mathrm{~F} 2 \mathrm{~V}$ & $\mathrm{H}$ \\
\hline 51986 & 92139 & $\ldots$ & $\mathrm{C}, \mathrm{A}$ & 26.840 & 3.830 & 0.299 & 7274 & A3 & $\mathrm{H}$ \\
\hline 52727 & 93497 & $\ldots$ & $\mathrm{C}$ & 35.900 & 2.800 & 0.900 & $\ldots$ & G5III & B \\
\hline 53229 & 94264 & $\ldots$ & $\mathrm{C}$ & 29.090 & 3.790 & 1.040 & 4833 & K0III-IV & $\mathrm{E}$ \\
\hline 53253 & 94510 & 404.1 & $\mathrm{C}$ & 29.130 & 3.780 & 0.945 & 5014 & K1III & $\mathrm{S}$ \\
\hline 53910 & 95418 & $\ldots$ & $\mathrm{C}, \mathrm{A}$ & 24.450 & 2.350 & 0.026 & 9342 & A1V & $\mathrm{H}$ \\
\hline 54182 & 96097 & $\cdots$ & $\mathrm{C}, \mathrm{A}$ & 28.990 & 4.620 & 0.332 & 7010 & F2III-IVvar & $\mathrm{H}$ \\
\hline
\end{tabular}


Table B.1-Continued

\begin{tabular}{|c|c|c|c|c|c|c|c|c|c|}
\hline $\begin{array}{l}\text { Hipp. } \\
\text { No. }\end{array}$ & $\begin{array}{l}\text { HD } \\
\text { No. }\end{array}$ & $\begin{array}{l}\text { Gliese } \\
\text { No. }\end{array}$ & $\begin{array}{c}\text { Target }^{\mathrm{a}} \\
\text { List }\end{array}$ & $\begin{array}{l}\text { Dist. } \\
\text { (pc) }\end{array}$ & $\begin{array}{c}V \\
(\mathrm{mag})\end{array}$ & $\begin{array}{c}B-V \\
(\mathrm{mag})\end{array}$ & $\begin{array}{l}T_{\text {eff }} \\
(\mathrm{K})\end{array}$ & Sp. T. & Notes \\
\hline 54872 & 97603 & 419 & $\mathrm{C}, \mathrm{A}$ & 17.910 & 2.560 & 0.128 & 8037 & $\mathrm{~A} 4 \mathrm{~V}$ & $\mathrm{H}$ \\
\hline 55642 & 99028 & $426.1 \mathrm{~A}$ & $\mathrm{C}, \mathrm{A}$ & 23.670 & 4.000 & 0.423 & 6600 & F2IV & $\mathrm{B}, \mathrm{H}$ \\
\hline 55705 & 99211 & $\ldots$ & $\mathrm{C}, \mathrm{A}$ & 25.240 & 4.070 & 0.216 & 7805 & A9V & $\mathrm{H}$ \\
\hline 55779 & 99453 & 3663 & $\mathrm{~A}$ & 27.220 & 5.180 & 0.500 & 6361 & F7V & $\mathrm{H}, \mathrm{S}$ \\
\hline 57632 & 102647 & 448 & $\mathrm{~S}, \mathrm{C}, \mathrm{A}$ & 11.000 & 2.140 & 0.090 & 8378 & A3Vvar & $\mathrm{H}$ \\
\hline 58001 & 103287 & $\ldots$ & $\mathrm{C}, \mathrm{A}$ & 25.500 & 2.390 & 0.045 & 9272 & $\mathrm{~A} 0 \mathrm{~V}$ & $\mathrm{~B}, \mathrm{H}$ \\
\hline 58803 & 104731 & 3701 & $\mathrm{~A}$ & 25.320 & 5.150 & 0.420 & 6638 & F5V & $\mathrm{H}, \mathrm{S}$ \\
\hline 59072 & 105211 & 455.2 & $\mathrm{C}, \mathrm{A}$ & 19.760 & 4.140 & 0.353 & 6950 & F0IV & $\mathrm{S}, \mathrm{H}$ \\
\hline 59199 & 105452 & 455.3 & $\mathrm{~S}, \mathrm{C}, \mathrm{A}$ & 14.940 & 4.020 & 0.334 & 7081 & F0IV/V & $\mathrm{H}$ \\
\hline 59774 & 106591 & 459 & $\mathrm{C}, \mathrm{A}$ & 24.690 & 3.280 & 0.077 & 8613 & A3Vvar & $\mathrm{H}$ \\
\hline 60965 & $108767 \mathrm{~A}$ & $\ldots$ & $\mathrm{C}, \mathrm{A}$ & 26.630 & 2.930 & -0.013 & 10207 & B9.5V & $\mathrm{H}$ \\
\hline 61084 & 108903 & 470 & $\mathrm{C}$ & 27.150 & 1.650 & 1.517 & 3385 & M4III & $\mathrm{S}$ \\
\hline 61174 & 109085 & 471.2 & $\mathrm{C}, \mathrm{A}$ & 18.280 & 4.300 & 0.388 & 6784 & $\mathrm{~F} 2 \mathrm{~V}$ & $\mathrm{H}$ \\
\hline 61941 & 110379 & $482 \mathrm{~A}$ & $\mathrm{~S}, \mathrm{C}, \mathrm{A}$ & 11.680 & 3.440 & 0.362 & 5674 & F1V & $\mathrm{H}$ \\
\hline 62956 & 112185 & $\ldots$ & $\mathrm{C}, \mathrm{A}$ & 25.310 & 1.760 & -0.022 & 9020 & A0p & $\mathrm{H}$ \\
\hline 63076 & 112429 & $\ldots$ & $\mathrm{A}$ & 29.290 & 5.230 & 0.290 & 7129 & F0V & $\mathrm{H}$ \\
\hline 63125 & 112413 & $\ldots$ & $\mathrm{C}$ & 35.200 & 2.800 & 0.120 & $\ldots$ & A0spe & $\mathrm{H}$ \\
\hline 63503 & 113139 & $\ldots$ & A & 25.440 & 4.930 & 0.370 & 6829 & $\mathrm{~F} 2 \mathrm{~V}$ & $\mathrm{H}$ \\
\hline 63608 & 113226 & $\ldots$ & $\mathrm{C}$ & 33.600 & 3.000 & 0.930 & $\ldots$ & G8IIIvar & $\mathrm{E}$ \\
\hline 63613 & 112985 & $\ldots$ & $\mathrm{C}$ & 27.870 & 3.600 & 1.189 & 4390 & K2III & $\mathrm{S}, \mathrm{E}$ \\
\hline 64241 & $\ldots$ & $\ldots$ & $\mathrm{C}, \mathrm{A}$ & 17.830 & 4.320 & 0.455 & 6399 & F5V & $\mathrm{H}$ \\
\hline 64583 & 114837 & 503 & $\mathrm{C}, \mathrm{A}$ & 18.200 & 4.910 & 0.469 & 6390 & F5V & $\mathrm{S}, \mathrm{H}$ \\
\hline 65109 & 115892 & 508.1 & $\mathrm{C}, \mathrm{A}$ & 18.020 & 2.720 & 0.068 & 10207 & $\mathrm{~A} 2 \mathrm{~V}$ & $\mathrm{H}$ \\
\hline 65378 & 116656 & $\ldots$ & $\mathrm{C}, \mathrm{A}$ & 26.310 & 2.220 & 0.051 & 9330 & $\mathrm{~A} 2 \mathrm{~V}$ & $\mathrm{H}$ \\
\hline 65477 & 116842 & $\ldots$ & $\mathrm{C}, \mathrm{A}$ & 25.060 & 3.980 & 0.169 & 7955 & $\mathrm{~A} 5 \mathrm{~V}$ & $\mathrm{~B}, \mathrm{H}$ \\
\hline 66249 & 118098 & $\ldots$ & $\mathrm{C}, \mathrm{A}$ & 22.710 & 3.380 & 0.114 & 8633 & $\mathrm{~A} 0 / 1 \mathrm{IV}$ & $\mathrm{H}$ \\
\hline 67153 & 119756 & 525.1 & $\mathrm{C}, \mathrm{A}$ & 19.400 & 4.230 & 0.375 & 6781 & F3V & $\mathrm{H}$ \\
\hline 67301 & 120315 & $\ldots$ & $\mathrm{C}$ & 31.900 & 1.800 & 0.100 & $\ldots$ & B3V & $\mathrm{B}, \mathrm{H}$ \\
\hline 67927 & 121370 & 534 & $\mathrm{~S}, \mathrm{C}, \mathrm{A}$ & 11.400 & 2.680 & 0.580 & 6116 & G0IV & B \\
\hline 68895 & 123123 & $\ldots$ & $\mathrm{C}$ & 31.000 & 3.400 & 1.090 & $\ldots$ & K2III & $\mathrm{E}$ \\
\hline 68933 & 123139 & 539 & $\mathrm{C}$ & 18.030 & 2.060 & 1.011 & 4823 & K0IIIB & $\mathrm{E}$ \\
\hline 69673 & 124897 & 541 & $\mathrm{C}$ & 11.260 & -0.050 & 1.239 & 4336 & K2IIIp & $\mathrm{E}$ \\
\hline 69701 & 124850 & $\ldots$ & $\mathrm{C}, \mathrm{A}$ & 22.240 & 4.070 & 0.511 & 6234 & F6III & $\mathrm{H}$ \\
\hline 69713 & 125161 & $\ldots$ & $\mathrm{A}$ & 29.070 & 4.750 & 0.240 & 7700 & A7IV & $\mathrm{H}$ \\
\hline 69732 & 125162 & $\ldots$ & $\mathrm{C}$ & 30.400 & 4.200 & 0.090 & $\ldots$ & A0sh & $\mathrm{H}$ \\
\hline 70497 & 126660 & $549 \mathrm{~A}$ & $\mathrm{~S}, \mathrm{C}, \mathrm{A}$ & 14.530 & 4.040 & 0.497 & 6192 & F7V & B \\
\hline
\end{tabular}


Table B.1-Continued

\begin{tabular}{|c|c|c|c|c|c|c|c|c|c|}
\hline $\begin{array}{l}\text { Hipp. } \\
\text { No. }\end{array}$ & $\begin{array}{l}\text { HD } \\
\text { No. }\end{array}$ & $\begin{array}{c}\text { Gliese } \\
\text { No. }\end{array}$ & $\begin{array}{c}\text { Target }^{\mathrm{a}} \\
\text { List }\end{array}$ & $\begin{array}{l}\text { Dist. } \\
\text { (pc) }\end{array}$ & $\begin{array}{c}V \\
(\mathrm{mag})\end{array}$ & $\begin{array}{l}B-V \\
(\mathrm{mag})\end{array}$ & $\begin{array}{l}T_{\text {eff }} \\
(\mathrm{K})\end{array}$ & Sp. T. & Notes $^{b}$ \\
\hline 71075 & 127762 & $\ldots$ & $\mathrm{C}, \mathrm{A}$ & 26.610 & 3.040 & 0.191 & 8047 & A7IIIvar & $\mathrm{H}$ \\
\hline 71681 & 128621 & $559 \mathrm{~B}$ & $\mathrm{~S}, \mathrm{C}, \mathrm{A}$ & 1.340 & 1.350 & 0.900 & 5178 & K0V & S \\
\hline 71683 & 128620 & $559 \mathrm{~A}$ & $\mathrm{~S}, \mathrm{C}, \mathrm{A}$ & 1.340 & -0.010 & 0.710 & 5801 & $\mathrm{G} 2.0 \mathrm{~V}$ & S \\
\hline 71908 & 128898 & $560 \mathrm{~A}$ & $\mathrm{~S}, \mathrm{C}, \mathrm{A}$ & 16.570 & 3.160 & 0.256 & 7631 & $\ldots$ & $\mathrm{S}, \mathrm{H}$ \\
\hline 71957 & 129502 & 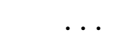 & $\mathrm{C}, \mathrm{A}$ & 18.270 & 3.860 & 0.385 & 6751 & $\mathrm{~F} 2 \mathrm{~V}$ & $\mathrm{H}$ \\
\hline 72603 & 130819 & 563.4 & $\mathrm{~A}$ & 22.980 & 5.150 & 0.400 & 6745 & $\mathrm{~F} 4 \mathrm{~V}$ & $\mathrm{H}$ \\
\hline 72622 & 130841 & 564.1 & $\mathrm{C}, \mathrm{A}$ & 23.240 & 2.750 & 0.147 & 8128 & A3III/V & $\mathrm{H}$ \\
\hline 72848 & 131511 & 567 & $\mathrm{~S}$ & 11.510 & 5.996 & 0.841 & 5335 & $\mathrm{~K} 2 \mathrm{~V}$ & $\mathrm{~B}$ ? \\
\hline 73165 & 132052 & $\ldots$ & $\mathrm{C}, \mathrm{A}$ & 26.900 & 4.460 & 0.318 & 7079 & $\mathrm{~F} 2 \mathrm{~V}$ & $\mathrm{H}$ \\
\hline 73695 & 133640 & $575 \mathrm{~A}$ & $\mathrm{~S}, \mathrm{C}, \mathrm{A}$ & 12.510 & 4.830 & 0.647 & 5533 & $\mathrm{G} 2 \mathrm{~V}+\mathrm{G} 2 \mathrm{~V}$ & B \\
\hline 74395 & 134505 & $\ldots$ & $\mathrm{C}$ & 36.000 & 3.600 & 0.920 & $\ldots$ & G8III & $\mathrm{S}$ \\
\hline 74605 & 136064 & 580.2 & $\mathrm{~A}$ & 25.340 & 5.150 & 0.540 & 6152 & F8V & $\mathrm{H}$ \\
\hline 74824 & 135379 & $\ldots$ & $\mathrm{C}$ & 30.600 & 4.100 & 0.090 & $\ldots$ & $\mathrm{A} 3 \mathrm{~V}$ & $\mathrm{~S}, \mathrm{H}$ \\
\hline 74975 & 136202 & $\ldots$ & $\mathrm{A}$ & 25.380 & 5.040 & 0.540 & 6119 & F8IV & $\mathrm{H}$ \\
\hline 75458 & 137759 & $\ldots$ & $\mathrm{C}$ & 31.000 & 3.500 & 1.170 & $\ldots$ & K2III & $\mathrm{E}$ \\
\hline 75695 & 137909 & $\ldots$ & $\mathrm{C}$ & 34.300 & 3.700 & 0.320 & $\ldots$ & F0p & $\mathrm{H}$ \\
\hline 76267 & 139006 & $\cdots$ & $\mathrm{C}, \mathrm{A}$ & 23.010 & 2.210 & 0.025 & 9584 & $\mathrm{~A} 0 \mathrm{~V}$ & $\mathrm{H}$ \\
\hline 76829 & 139664 & 594 & $\mathrm{C}, \mathrm{A}$ & 17.440 & 4.630 & 0.413 & 6649 & $\mathrm{~F} 3 / 5 \mathrm{~V}$ & $\mathrm{H}$ \\
\hline 77070 & 140573 & 596.2 & $\mathrm{C}$ & 22.680 & 2.610 & 1.167 & 4548 & K2III & $\mathrm{E}$ \\
\hline 77622 & 141795 & $\ldots$ & $\mathrm{C}, \mathrm{A}$ & 21.600 & 3.710 & 0.147 & 8257 & $\ldots$ & $\mathrm{H}$ \\
\hline 77952 & 141891 & $601 \mathrm{~A}$ & $\mathrm{~S}, \mathrm{C}, \mathrm{A}$ & 12.380 & 2.810 & 0.315 & 7109 & F0III/IV & $\mathrm{S}, \mathrm{H}$ \\
\hline 78527 & 144284 & 609.1 & $\mathrm{C}, \mathrm{A}$ & 21.030 & 3.990 & 0.528 & 4642 & F8IV-V & B \\
\hline 79822 & 148048 & $3950 \mathrm{~A}$ & A & 29.730 & 4.950 & 0.360 & 6868 & $\mathrm{~F} 2 \mathrm{~V}$ & $\mathrm{H}$ \\
\hline 79882 & 146791 & $\cdots$ & $\mathrm{C}$ & 32.600 & 3.400 & 0.970 & $\ldots$ & G8III & $\mathrm{E}$ \\
\hline 80179 & 147449 & $\ldots$ & $\mathrm{A}$ & 27.270 & 4.820 & 0.340 & 6981 & F0V & $\mathrm{H}$ \\
\hline 80331 & 148387 & $624.1 \mathrm{~A}$ & $\mathrm{C}$ & 28.230 & 2.730 & 0.910 & 4941 & G8III & B \\
\hline 80337 & 147513 & $620.1 \mathrm{~A}$ & $\mathrm{~A}$ & 12.780 & 5.370 & 0.630 & 5930 & G5V & $\mathrm{S}$ \\
\hline 80686 & 147584 & 624 & $\mathrm{~S}, \mathrm{C}, \mathrm{A}$ & 12.120 & 4.900 & 0.555 & 6107 & G0V & $\mathrm{S}$ \\
\hline 81693 & 150680 & $635 \mathrm{~A}$ & $\mathrm{~S}, \mathrm{C}, \mathrm{A}$ & 10.720 & 2.810 & 0.650 & 5820 & F9IV & B \\
\hline 81833 & 150997 & $\ldots$ & $\mathrm{C}$ & 33.300 & 3.600 & 0.920 & $\ldots$ & G8III-IV & $\mathrm{E}$ \\
\hline 82020 & 151613 & $\cdots$ & $\mathrm{A}$ & 26.730 & 4.840 & 0.380 & 6805 & $\mathrm{~F} 2 \mathrm{~V}$ & $\mathrm{H}$ \\
\hline 82396 & 151680 & 639.1 & $\mathrm{C}$ & 19.540 & 2.260 & 1.181 & 4703 & K2IIIB & $\mathrm{E}$ \\
\hline 82587 & 152598 & $\ldots$ & $\mathrm{A}$ & 29.190 & 5.340 & 0.310 & 7136 & F0V & $\mathrm{H}$ \\
\hline 82860 & 153597 & 648 & $\mathrm{C}, \mathrm{A}$ & 15.260 & 4.880 & 0.481 & 6146 & F6Vvar & B \\
\hline 83000 & 153210 & $\cdots$ & $\mathrm{C}$ & 28.040 & 3.180 & 1.158 & 4564 & K2IIIvar & $\mathrm{E}$ \\
\hline 83431 & 153580 & $\ldots$ & $\mathrm{A}$ & 27.220 & 5.270 & 0.470 & 6501 & F5V & $\mathrm{H}, \mathrm{S}$ \\
\hline
\end{tabular}


Table B.1-Continued

\begin{tabular}{|c|c|c|c|c|c|c|c|c|c|}
\hline $\begin{array}{l}\text { Hipp. } \\
\text { No. }\end{array}$ & $\begin{array}{l}\text { HD } \\
\text { No. }\end{array}$ & $\begin{array}{c}\text { Gliese } \\
\text { No. }\end{array}$ & $\begin{array}{c}\text { Target }^{\mathrm{a}} \\
\text { List }\end{array}$ & $\begin{array}{l}\text { Dist. } \\
\text { (pc) }\end{array}$ & $\begin{array}{c}V \\
(\mathrm{mag})\end{array}$ & $\begin{array}{c}B-V \\
(\mathrm{mag})\end{array}$ & $\begin{array}{l}T_{\text {eff }} \\
(\mathrm{K})\end{array}$ & Sp. T. & Notes $^{\mathrm{b}}$ \\
\hline 84012 & 155125 & $656.1 \mathrm{~A}$ & $\mathrm{C}, \mathrm{A}$ & 27.090 & 2.430 & 0.059 & 8788 & $\mathrm{~A} 2.5 \mathrm{VA}$ & $\mathrm{H}$ \\
\hline 84143 & 155203 & 657 & $\mathrm{C}, \mathrm{A}$ & 22.530 & 3.310 & 0.423 & 6519 & $\mathrm{~F} 2 \mathrm{~V}$ & $\mathrm{H}$ \\
\hline 84379 & 156164 & $\cdots$ & $\mathrm{C}, \mathrm{A}$ & 23.040 & 3.120 & 0.080 & 8879 & $\mathrm{~A} 3 \mathrm{IVv}$ & $\mathrm{B}, \mathrm{H}$ \\
\hline 84405 & 155885 & $663 \mathrm{~A}$ & $\mathrm{~S}, \mathrm{C}, \mathrm{A}$ & 5.950 & 4.330 & 0.855 & 5119 & $\mathrm{~K} 1.5 \mathrm{~V}$ & B \\
\hline 84709 & $\ldots$ & $667 \mathrm{AB}$ & $\mathrm{S}$ & 6.836 & 6.255 & 0.999 & 4810 & $\mathrm{M} 1.5 \mathrm{~V}$ & $\mathrm{~S}$ \\
\hline 84720 & $\ldots$ & $666 \mathrm{AB}$ & $\mathrm{S}, \mathrm{C}$ & 8.800 & 5.520 & 0.791 & 5052 & M0V & $\mathrm{S}$ \\
\hline 84893 & 156897 & $670 \mathrm{~A}$ & $\mathrm{C}, \mathrm{A}$ & 17.360 & 4.390 & 0.366 & 6704 & $\mathrm{~F} 2 / 3 \mathrm{~V}$ & $\mathrm{H}$ \\
\hline 85340 & 157792 & 673.1 & $\mathrm{C}, \mathrm{A}$ & 25.500 & 4.150 & 0.283 & 7440 & $\mathrm{~A} 3$ & $\mathrm{H}$ \\
\hline 85667 & 158614 & $678 \mathrm{~A}$ & $\mathrm{~S}$ & 16.340 & 5.310 & 0.715 & 5538 & G6V & B \\
\hline 86032 & 159561 & 681 & $\mathrm{~S}, \mathrm{C}$ & 14.900 & 2.080 & 0.155 & 8225 & A5III & $\mathrm{H}$ \\
\hline 86036 & 160269 & $684 \mathrm{~A}$ & A & 14.190 & 5.230 & 0.600 & 5898 & G0V & B \\
\hline 86201 & 160922 & $\ldots$ & $\mathrm{C}$ & 23.160 & 4.770 & 0.430 & 6560 & F5V & $\mathrm{H}$ \\
\hline 86486 & 160032 & 686.2 & $\mathrm{C}$ & 21.450 & 4.750 & 0.403 & 6678 & $\mathrm{~F} 2 \mathrm{~V}$ & $\mathrm{H}$ \\
\hline 86614 & 162003 & $694.1 \mathrm{~A}$ & $\mathrm{C}$ & 22.840 & 4.550 & 0.433 & 6435 & F5IV-V & $\mathrm{H}$ \\
\hline 86736 & 160915 & 692 & $\mathrm{C}$ & 17.650 & 4.860 & 0.469 & 6465 & $\mathrm{~F} 6 / 7 \mathrm{~V}$ & $\mathrm{H}$ \\
\hline 86742 & 161096 & $\ldots$ & $\mathrm{C}$ & 25.090 & 2.750 & 1.191 & 4571 & K2III & $\mathrm{E}$ \\
\hline 86796 & 160691 & 691 & $\mathrm{~A}$ & 15.510 & 5.120 & 0.690 & 5784 & G3IV-V & $\mathrm{S}$ \\
\hline 87108 & 161868 & $\ldots$ & $\mathrm{C}$ & 31.500 & 3.800 & 0.040 & $\ldots$ & $\mathrm{A} 0 \mathrm{~V}$ & $\mathrm{H}$ \\
\hline 87585 & 163588 & $\ldots$ & $\mathrm{C}$ & 34.500 & 3.900 & 1.180 & $\ldots$ & K2III & $\mathrm{E}$ \\
\hline 88175 & 164259 & 699.2 & $\mathrm{C}, \mathrm{A}$ & 23.550 & 4.620 & 0.367 & 6771 & F2IV & $\mathrm{H}$ \\
\hline 88601 & 165341 & $702 \mathrm{~A}$ & $\mathrm{~S}, \mathrm{C}, \mathrm{A}$ & 5.100 & 4.030 & 0.860 & 5019 & K0V & $\mathrm{E}$ \\
\hline 88635 & 165135 & & $\mathrm{C}$ & 29.700 & 2.950 & 1.021 & 4914 & K0III & $\mathrm{E}$ \\
\hline 88745 & 165908 & $704 \mathrm{~A}$ & $\mathrm{~S}, \mathrm{~A}$ & 15.640 & 5.070 & 0.504 & 5925 & F7V & B \\
\hline 88771 & 165777 & $\ldots$ & $\mathrm{C}, \mathrm{A}$ & 26.630 & 3.700 & 0.140 & 8400 & $\mathrm{~A} 4 \mathrm{IVs}$ & $\mathrm{H}$ \\
\hline 89348 & 168151 & 708.1 & $\mathrm{~A}$ & 22.920 & 4.990 & 0.430 & 6404 & $\mathrm{~F} 5 \mathrm{~V}$ & $\mathrm{H}$ \\
\hline 89937 & 170153 & $713 \mathrm{AB}$ & $\mathrm{C}, \mathrm{A}$ & 8.060 & 3.560 & 0.489 & 6122 & F7Vvar & B \\
\hline 90139 & 169414 & $\ldots$ & $\mathrm{C}$ & 36.500 & 4.000 & 1.170 & $\ldots$ & K2III & $\mathrm{E}$ \\
\hline 90496 & 169916 & 713.1 & $\mathrm{C}$ & 23.970 & 2.810 & 1.057 & 4809 & K1IIIB & $\mathrm{E}$ \\
\hline 91262 & 172167 & 721 & $\mathrm{~S}, \mathrm{C}, \mathrm{A}$ & 7.680 & 0.030 & -0.001 & 9519 & A0Vvar & $\mathrm{H}$ \\
\hline 92024 & 172555 & $\ldots$ & $\mathrm{A}$ & 28.550 & 4.780 & 0.200 & 7846 & $\mathrm{~A} 7 \mathrm{~V}$ & $\mathrm{H}, \mathrm{S}$ \\
\hline 92161 & 173880 & $\ldots$ & $\mathrm{C}, \mathrm{A}$ & 28.890 & 4.340 & 0.127 & 8334 & A5III & $\mathrm{H}$ \\
\hline 93017 & 176051 & $738 \mathrm{~A}$ & $\mathrm{~S}, \mathrm{~A}$ & 14.870 & 5.280 & 0.555 & 6064 & G0V & B \\
\hline 93506 & 176687 & $\ldots$ & $\mathrm{C}$ & 27.040 & 2.600 & 0.062 & 8799 & A3IV & $\mathrm{H}$ \\
\hline 93747 & 177724 & $\ldots$ & $\mathrm{C}, \mathrm{A}$ & 25.460 & 2.990 & 0.014 & 9190 & $\mathrm{~A} 0 \mathrm{Vn}$ & $\mathrm{H}$ \\
\hline 93825 & 177474 & $743.1 \mathrm{~A}$ & $\mathrm{C}, \mathrm{A}$ & 17.300 & 4.230 & 0.523 & 6202 & $\mathrm{~F} 8 / \mathrm{G} 0 \mathrm{~V}$ & $\mathrm{~B}$ \\
\hline 94083 & 180777 & 748.1 & $\mathrm{~A}$ & 27.300 & 5.110 & 0.310 & 7129 & FoVs & $\mathrm{H}$ \\
\hline
\end{tabular}


Table B.1-Continued

\begin{tabular}{|c|c|c|c|c|c|c|c|c|c|}
\hline $\begin{array}{l}\text { Hipp. } \\
\text { No. }\end{array}$ & $\begin{array}{l}\text { HD } \\
\text { No. }\end{array}$ & $\begin{array}{c}\text { Gliese } \\
\text { No. }\end{array}$ & $\begin{array}{c}\text { Target }^{\mathrm{a}} \\
\text { List }\end{array}$ & $\begin{array}{l}\text { Dist. } \\
\text { (pc) }\end{array}$ & $\begin{array}{c}V \\
(\mathrm{mag})\end{array}$ & $\begin{array}{c}B-V \\
(\mathrm{mag})\end{array}$ & $\begin{array}{l}T_{\text {eff }} \\
(\mathrm{K})\end{array}$ & Sp. T. & Notes $^{\mathrm{b}}$ \\
\hline 94376 & & & $\mathrm{C}$ & 29.870 & 3.050 & 1.001 & 4966 & G9III & $\mathrm{E}$ \\
\hline 95501 & 182640 & 760 & $\mathrm{~S}, \mathrm{C}, \mathrm{A}$ & 15.530 & 3.360 & 0.319 & 7074 & F2IV & $\mathrm{H}$ \\
\hline 97295 & 187013 & $767.1 \mathrm{~A}$ & $\mathrm{~A}$ & 21.230 & 5.000 & 0.470 & 6401 & F5.5IV-V & $\mathrm{H}, \mathrm{B}$ \\
\hline 97649 & 187642 & 768 & $\mathrm{~S}, \mathrm{C}, \mathrm{A}$ & 5.120 & 0.760 & 0.221 & 7800 & A7IV-V & $\mathrm{H}$ \\
\hline 97650 & 187532 & $\ldots$ & $\mathrm{A}$ & 27.870 & 5.380 & 0.350 & 6812 & F5V & $\mathrm{H}, \mathrm{B}$ \\
\hline 98066 & 188376 & 770.1 & $\mathrm{~A}$ & 25.840 & 4.700 & 0.750 & 5425 & G5IV & $\mathrm{B}, N_{\mathrm{obs}}=13$ \\
\hline 98495 & 188228 & $\ldots$ & $\mathrm{C}$ & 32.200 & 4.000 & 0.030 & $\ldots$ & $\mathrm{A} 0 \mathrm{~V}$ & $\mathrm{~S}, \mathrm{H}$ \\
\hline 99240 & 190248 & 780 & $\mathrm{~S}, \mathrm{C}, \mathrm{A}$ & 6.110 & 3.530 & 0.765 & 5590 & G8.0IV & $\mathrm{S}$ \\
\hline 101612 & 195627 & $\ldots$ & A & 27.790 & 4.750 & 0.290 & 7201 & F0V & $\mathrm{H}, \mathrm{S}$ \\
\hline 101983 & 196378 & 794.2 & $\mathrm{~A}$ & 24.660 & 5.110 & 0.510 & 6040 & G0V & $\mathrm{S}$ \\
\hline 102333 & 197157 & $\ldots$ & $\mathrm{C}, \mathrm{A}$ & 24.170 & 4.500 & 0.278 & 7448 & A7III/IV & $\mathrm{S}, \mathrm{H}$ \\
\hline 102422 & 198149 & 807 & $\mathrm{~S}, \mathrm{C}$ & 14.270 & 3.410 & 0.912 & 4940 & K0IV & $\mathrm{E}$ \\
\hline 102431 & 198084 & $\ldots$ & $\mathrm{C}, \mathrm{A}$ & 27.290 & 4.520 & 0.535 & 6138 & F8IV-V & B \\
\hline 102485 & 197692 & 805 & $\mathrm{~S}, \mathrm{C}, \mathrm{A}$ & 14.680 & 4.130 & 0.426 & 6633 & F5V & $\mathrm{H}$ \\
\hline 102488 & 197989 & $806.1 \mathrm{~A}$ & $\mathrm{C}$ & 22.290 & 2.450 & 1.021 & 4799 & KOIII & $\mathrm{E}$ \\
\hline 104858 & 202275 & $822 \mathrm{~A}$ & $\mathrm{C}, \mathrm{A}$ & 18.490 & 4.470 & 0.529 & 6238 & F5V & $\mathrm{H}$ \\
\hline 104887 & 202444 & $822.1 \mathrm{~A}$ & $\mathrm{C}, \mathrm{A}$ & 20.340 & 3.740 & 0.393 & 6621 & F1IV & $\mathrm{H}$ \\
\hline 105199 & 203280 & 826 & $\mathrm{~S}, \mathrm{C}, \mathrm{A}$ & 15.040 & 2.430 & 0.243 & 7773 & A7IV-V & $\mathrm{H}$ \\
\hline 105858 & 203608 & 827 & $\mathrm{~S}, \mathrm{C}, \mathrm{A}$ & 9.260 & 4.220 & 0.469 & 6205 & F7V & S \\
\hline 107089 & 205478 & 835.1 & $\mathrm{C}$ & 21.200 & 3.730 & 1.017 & 4769 & KOIII & S \\
\hline 107310 & 206826 & $836.6 \mathrm{~A}$ & $\mathrm{C}, \mathrm{A}$ & 22.240 & 4.690 & 0.454 & 6309 & F6V & B \\
\hline 107556 & $\ldots$ & $\ldots$ & $\mathrm{S}, \mathrm{C}, \mathrm{A}$ & 11.870 & 2.850 & 0.305 & 7301 & $\mathrm{~A} 5 \mathrm{mF} 2$ & $\mathrm{H}$ \\
\hline 107649 & 207129 & 838 & $\mathrm{~S}$ & 15.990 & 5.570 & 0.601 & 5889 & G0V & $\mathrm{Y}$ \\
\hline 108036 & 207958 & 838.5 & $\mathrm{~A}$ & 26.610 & 5.080 & 0.380 & 6799 & $\mathrm{~F} 2 \mathrm{~V}$ & $\mathrm{H}$ \\
\hline 108870 & 209100 & 845 & $\mathrm{~S}, \mathrm{C}, \mathrm{A}$ & 3.620 & 4.690 & 1.056 & 4683 & $\mathrm{~K} 4 \mathrm{~V}$ & $\mathrm{Y}, \mathrm{S}$ \\
\hline 108917 & & & $\mathrm{C}, \mathrm{A}$ & 29.590 & 4.400 & 0.341 & 6964 & $\mathrm{Am}$ & $\mathrm{H}$ \\
\hline 109176 & 210027 & 848 & $\mathrm{~S}, \mathrm{C}, \mathrm{A}$ & 11.730 & 3.770 & 0.435 & 6442 & F5V & $\mathrm{H}$ \\
\hline 109268 & 209952 & $\ldots$ & $\mathrm{C}$ & 31.000 & 1.700 & 0.070 & $\ldots$ & B7IV & $\mathrm{H}$ \\
\hline 109427 & 210418 & $\ldots$ & $\mathrm{C}, \mathrm{A}$ & 28.300 & 3.520 & 0.086 & 8569 & $\mathrm{~A} 2 \mathrm{~V}$ & $\mathrm{H}$ \\
\hline 109857 & 211336 & $\ldots$ & $\mathrm{C}, \mathrm{A}$ & 26.200 & 4.170 & 0.278 & 7283 & F0IV & $\mathrm{H}$ \\
\hline 110109 & 211415 & $853 \mathrm{~A}$ & $\mathrm{~S}, \mathrm{~A}$ & 13.790 & 5.360 & 0.614 & 5837 & G3V & S \\
\hline 110618 & 211998 & $855.1 \mathrm{~A}$ & $\mathrm{~A}$ & 28.700 & 5.280 & 0.630 & 5486 & G9V & S \\
\hline 110649 & 212330 & 857 & $\mathrm{~A}$ & 20.560 & 5.310 & 0.670 & 5739 & G2IV-V & S \\
\hline 110960 & $\ldots$ & $\ldots$ & $\mathrm{C}$ & 28.170 & 3.650 & 0.406 & 6619 & F3III-IV & $\mathrm{H}, \mathrm{E}$ \\
\hline 111169 & 213558 & $\cdots$ & $\mathrm{C}$ & 31.500 & 3.800 & 0.030 & $\ldots$ & A1V & $\mathrm{H}$ \\
\hline 111449 & 213845 & 863.2 & $\mathrm{~A}$ & 22.680 & 5.210 & 0.450 & 6597 & F5V & $\mathrm{H}$ \\
\hline
\end{tabular}




\section{Completeness of Individual Stars}

In this appendix show the results of automated searches for planets in our Doppler data. This search methodology is described fully in Sec. 5.1. We also show the completeness limits for each star computed by injection-recovery tests (Sec. 5.3). The figures below show these results in graphical form for the 76 stars for which we have Doppler data. Each figure caption lists the HD number, Hipparcos number, and the codes for the imaging program target lists ( $\mathrm{S}=$ starshade, $\mathrm{C}=$ coronagraph, $\mathrm{A}=$ WFIRST-AFTA). 
Table B.1-Continued

\begin{tabular}{|c|c|c|c|c|c|c|c|c|c|}
\hline $\begin{array}{l}\text { Hipp. } \\
\text { No. }\end{array}$ & $\begin{array}{l}\text { HD } \\
\text { No. }\end{array}$ & $\begin{array}{c}\text { Gliese } \\
\text { No. }\end{array}$ & $\begin{array}{c}\text { Target }^{\mathrm{a}} \\
\text { List }\end{array}$ & $\begin{array}{l}\text { Dist. } \\
\text { (pc) }\end{array}$ & $\begin{array}{c}V \\
(\mathrm{mag})\end{array}$ & $\begin{array}{c}B-V \\
(\mathrm{mag})\end{array}$ & $\begin{array}{l}T_{\text {eff }} \\
(\mathrm{K})\end{array}$ & Sp. T. & Notes \\
\hline 112724 & 216228 & $\ldots$ & $\mathrm{C}$ & 35.300 & 3.700 & 1.050 & $\ldots$ & K0III & $\mathrm{E}$ \\
\hline 112748 & 216131 & $\ldots$ & $\mathrm{C}$ & 32.500 & 3.700 & 0.930 & $\ldots$ & M2III & $\mathrm{E}$ \\
\hline 112935 & 216385 & $9801 \mathrm{~A}$ & $\mathrm{~A}$ & 27.280 & 5.160 & 0.470 & 6250 & F6V & $\mathrm{H}$ \\
\hline 113368 & 216956 & $881 \mathrm{~A}$ & $\mathrm{~S}, \mathrm{C}, \mathrm{A}$ & 7.700 & 1.230 & 0.140 & 8399 & $\mathrm{~A} 3 \mathrm{~V}$ & $\mathrm{H}$ \\
\hline 113638 & 217364 & $\ldots$ & $\mathrm{C}$ & 33.400 & 4.300 & 0.960 & $\ldots$ & G8III & $\mathrm{S}$ \\
\hline 113860 & 217792 & 886.2 & $\mathrm{~A}$ & 29.400 & 5.120 & 0.280 & 7143 & F1V & $\mathrm{H}, \mathrm{S}$ \\
\hline 114570 & 219080 & 891.1 & $\mathrm{C}, \mathrm{A}$ & 24.590 & 4.520 & 0.302 & 7176 & F0V & $\mathrm{H}$ \\
\hline 114996 & 219571 & $\ldots$ & $\mathrm{C}, \mathrm{A}$ & 23.060 & 3.990 & 0.410 & 6618 & F3IV/V & $\mathrm{S}, \mathrm{H}$ \\
\hline 115126 & 219834 & $\ldots$ & $\mathrm{A}$ & 21.050 & 5.200 & 0.790 & 5461 & G8.5IV & \\
\hline 116584 & 222107 & $\ldots$ & $\mathrm{C}$ & 26.410 & 3.850 & 0.987 & 4636 & G8III-IV & B \\
\hline 116727 & 222404 & 903 & $\mathrm{C}$ & 14.100 & 3.210 & 1.031 & 4761 & K1IV & $\mathrm{E}$ \\
\hline 118268 & 224617 & $\ldots$ & $\mathrm{C}$ & 32.000 & 4.100 & 0.420 & $\ldots$ & F4IV & $\mathrm{H}$ \\
\hline
\end{tabular}

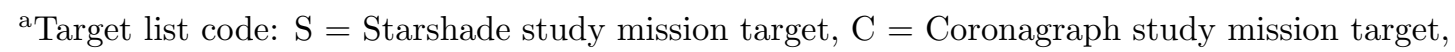
A = WFIRST-AFTA study mission target.

${ }^{\mathrm{b}}$ Stars were not observed by Lick and Keck Doppler programs because of these (non-exhaustive) list of reasons: star too hot (H), typically earlier than F8V; star too far south (S); star is evolved (E) into a giant or subgiant; star too young $(\mathrm{Y})$, with chromospheric activity substantially increasing jitter; or binary (B) or higher stellar multiple noted in the literature and/or our RVs. These reasons for a lack of observations were inferred in 2014 based on stellar properties and not based on a target down-selections when observing lists were created. 

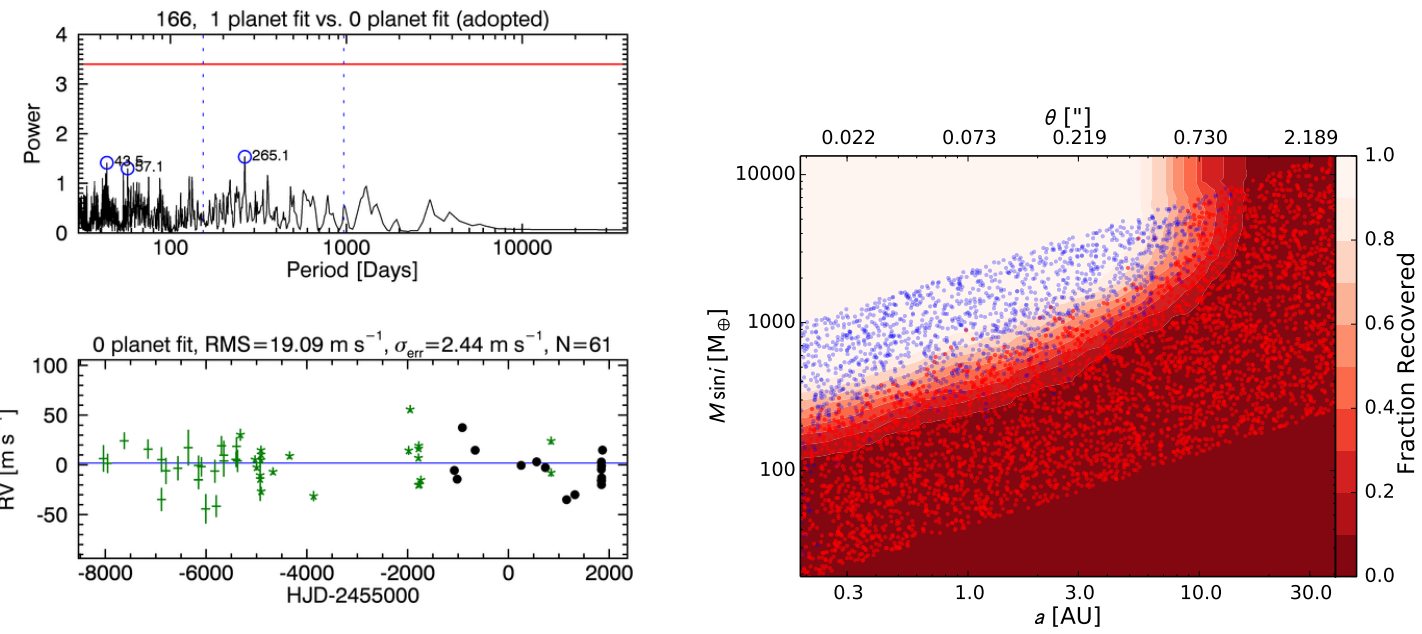

Fig. C.1.- Results from an automated search for planets orbiting the star HD 166 (HIP 544; program $=\mathrm{S}$ ) based on RVs from Lick and/or Keck Observatory. The set of plots on the left (analogous to Figures 4 and 5) show the planet search results and the plot on the right shows the completeness limits (analogous to Fig. 6). See the captions of those figures for detailed descriptions. 

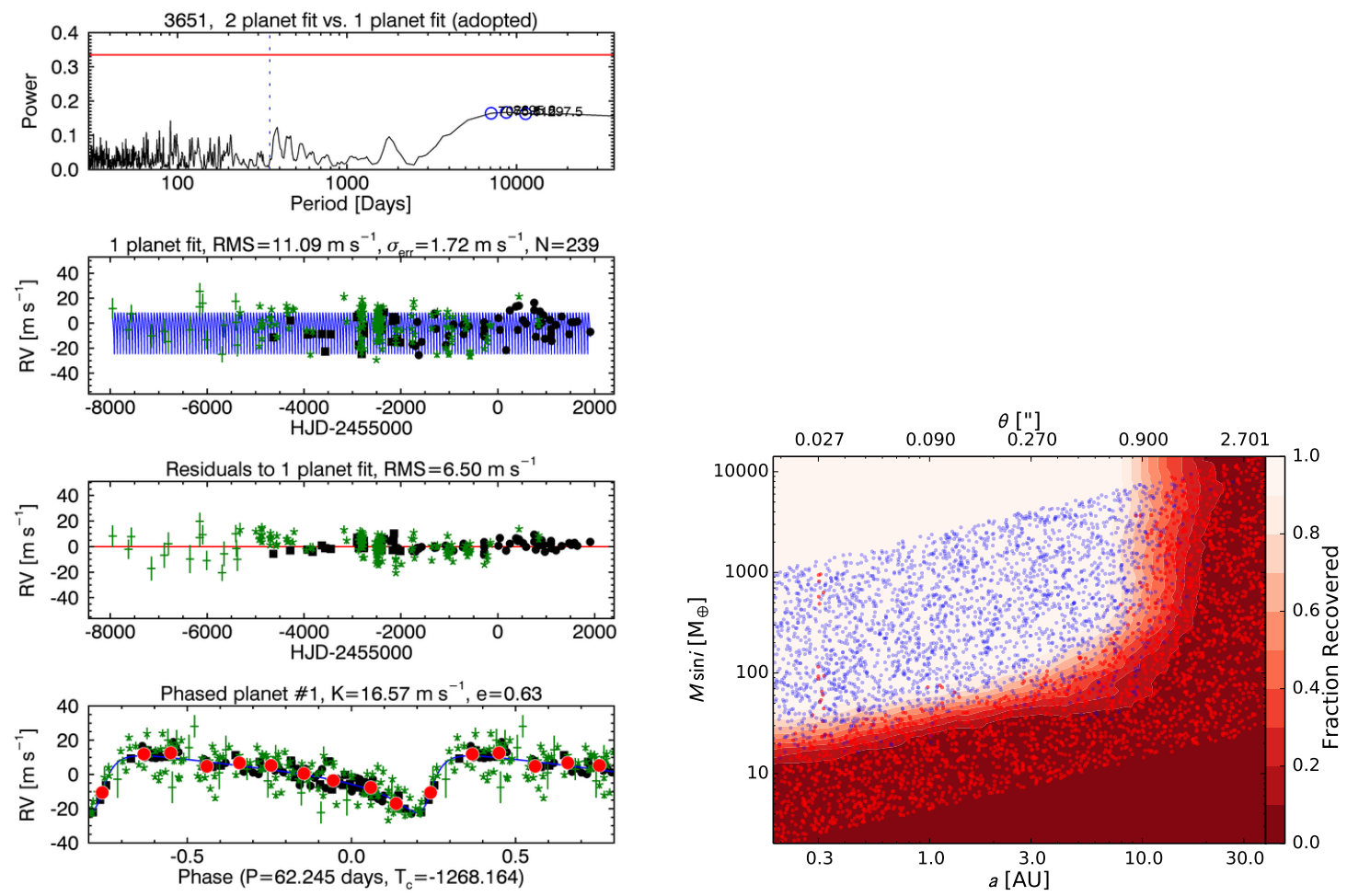

Fig. C.2.- Results from an automated search for planets orbiting the star HD 3651 (HIP 3093; program $=\mathrm{S}$ ) based on RVs from Lick and/or Keck Observatory. The set of plots on the left (analogous to Figures 4 and 5) show the planet search results and the plot on the right shows the completeness limits (analogous to Fig. 6). See the captions of those figures for detailed descriptions. This star has one known planet. 

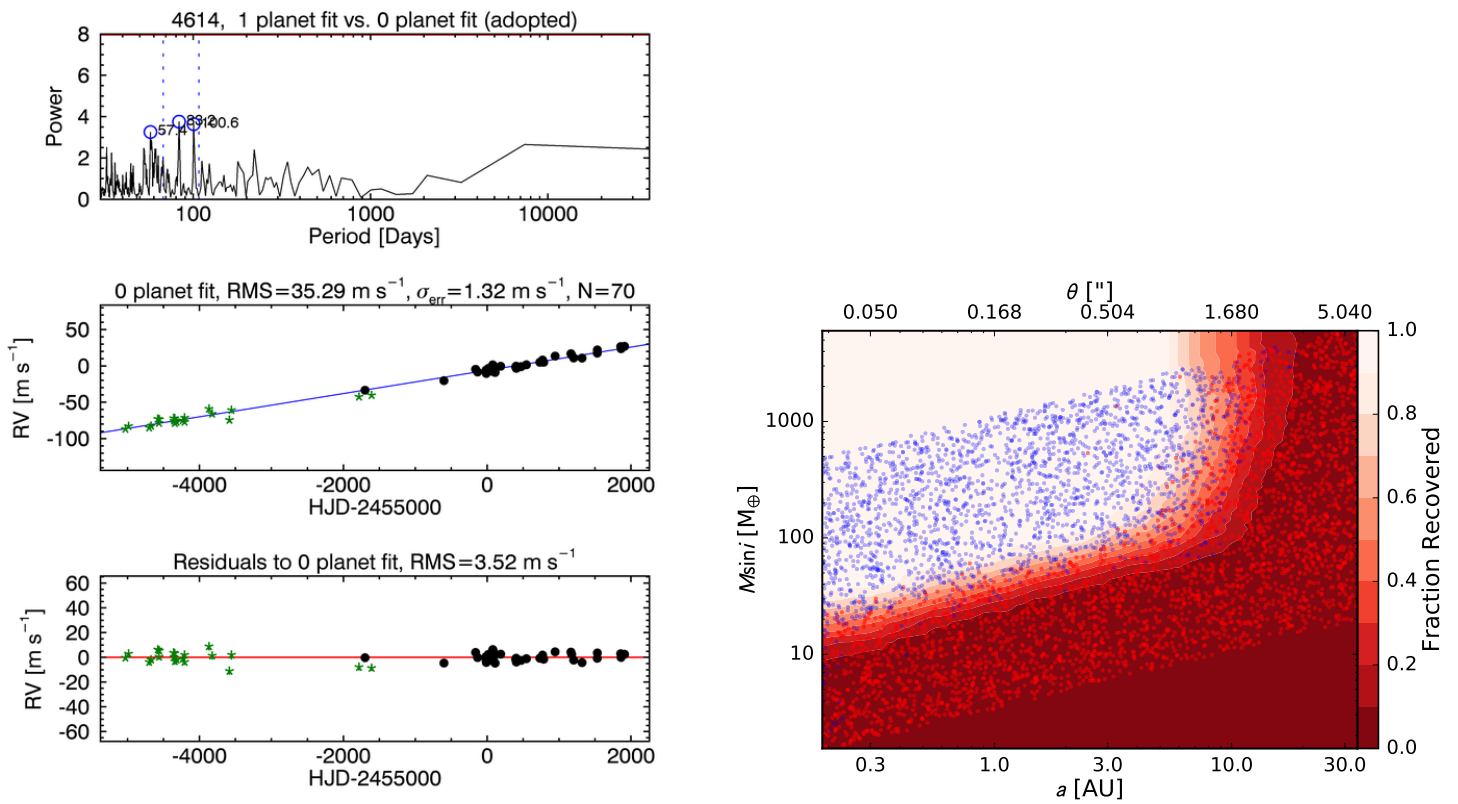

Fig. C.3.- Results from an automated search for planets orbiting the star HD 4614 (HIP 3821; programs = S, C, A) based on RVs from Lick and/or Keck Observatory. The set of plots on the left (analogous to Figures 4 and 5 show the planet search results and the plot on the right shows the completeness limits (analogous to Fig. 6). See the captions of those figures for detailed descriptions. This star shows a significant linear trend with no detectable curvature, presumably due to its known stellar companion. 

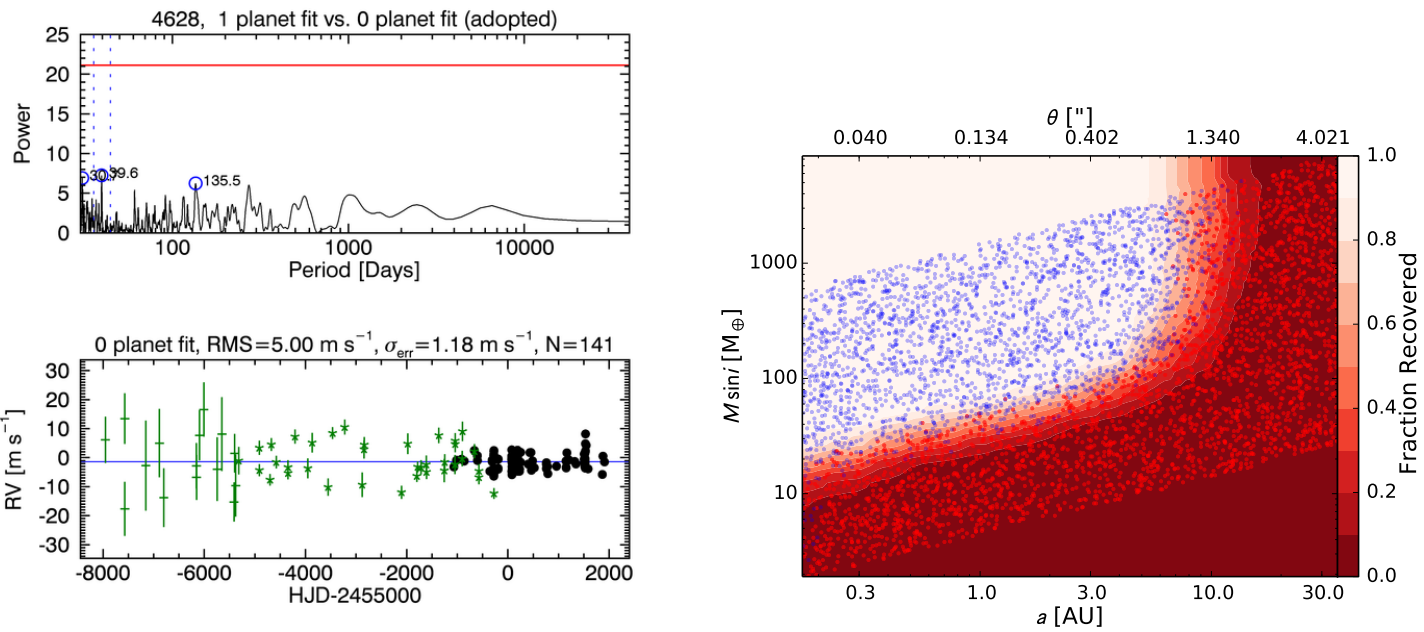

Fig. C.4. - Results from an automated search for planets orbiting the star HD 4628 (HIP 3765; programs $=$ S, C) based on RVs from Lick and/or Keck Observatory. The set of plots on the left (analogous to Figures 4 and 5 show the planet search results and the plot on the right shows the completeness limits (analogous to Fig. 6). See the captions of those figures for detailed descriptions. 

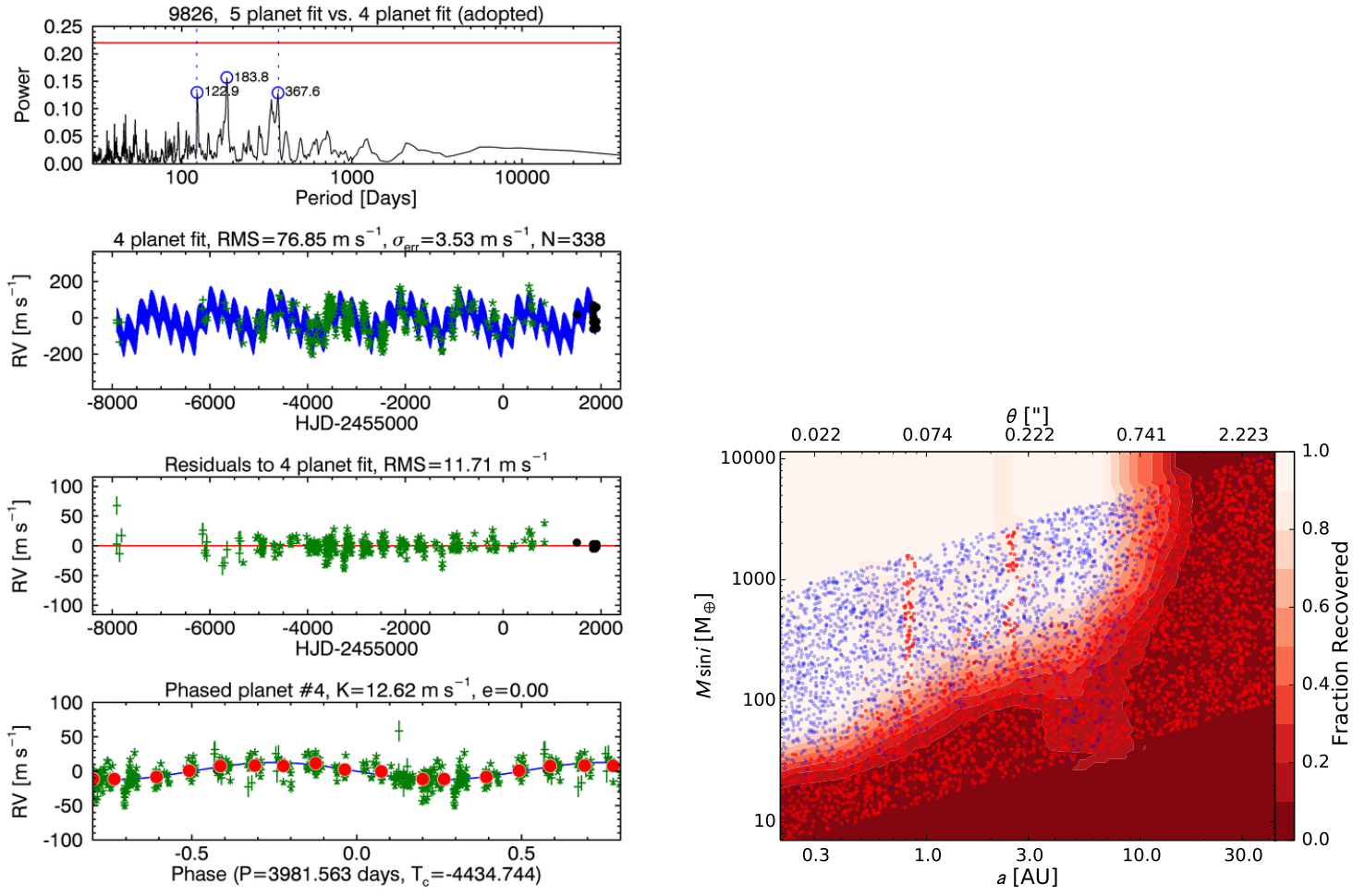

Fig. C.5.- Results from an automated search for planets orbiting the star HD 9826 (HIP 7513; programs $=\mathrm{C}$, A) based on RVs from Lick and/or Keck Observatory. The set of plots on the left (analogous to Figures 4 and 5) show the planet search results and the plot on the right shows the completeness limits (analogous to Fig. 6). See the captions of those figures for detailed descriptions. This star has three known planets. We detect an additional periodicity at $\sim 4000$ days and interpret this as the signature of a stellar magnetic activity cycle. 

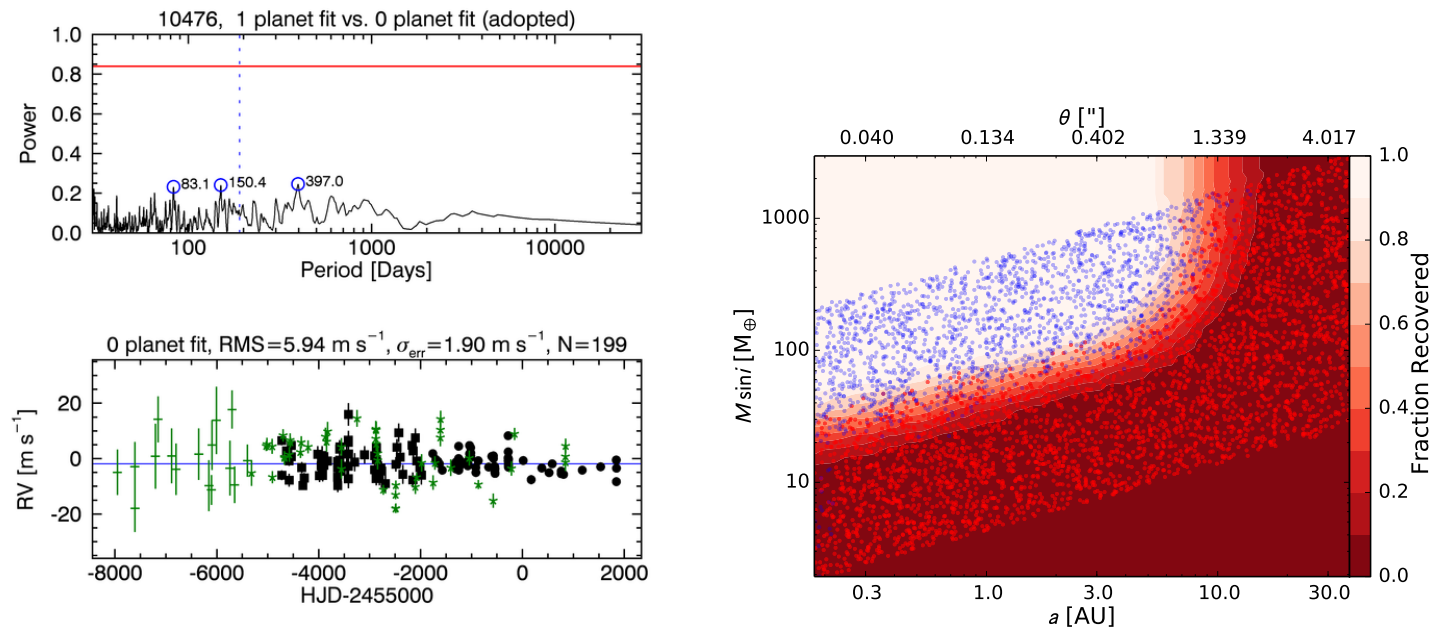

Fig. C.6. - Results from an automated search for planets orbiting the star HD 10476 (HIP 7981; programs = S, C, A) based on RVs from Lick and/or Keck Observatory. The set of plots on the left (analogous to Figures 4 and 5) show the planet search results and the plot on the right shows the completeness limits (analogous to Fig. 6). See the captions of those figures for detailed descriptions. 

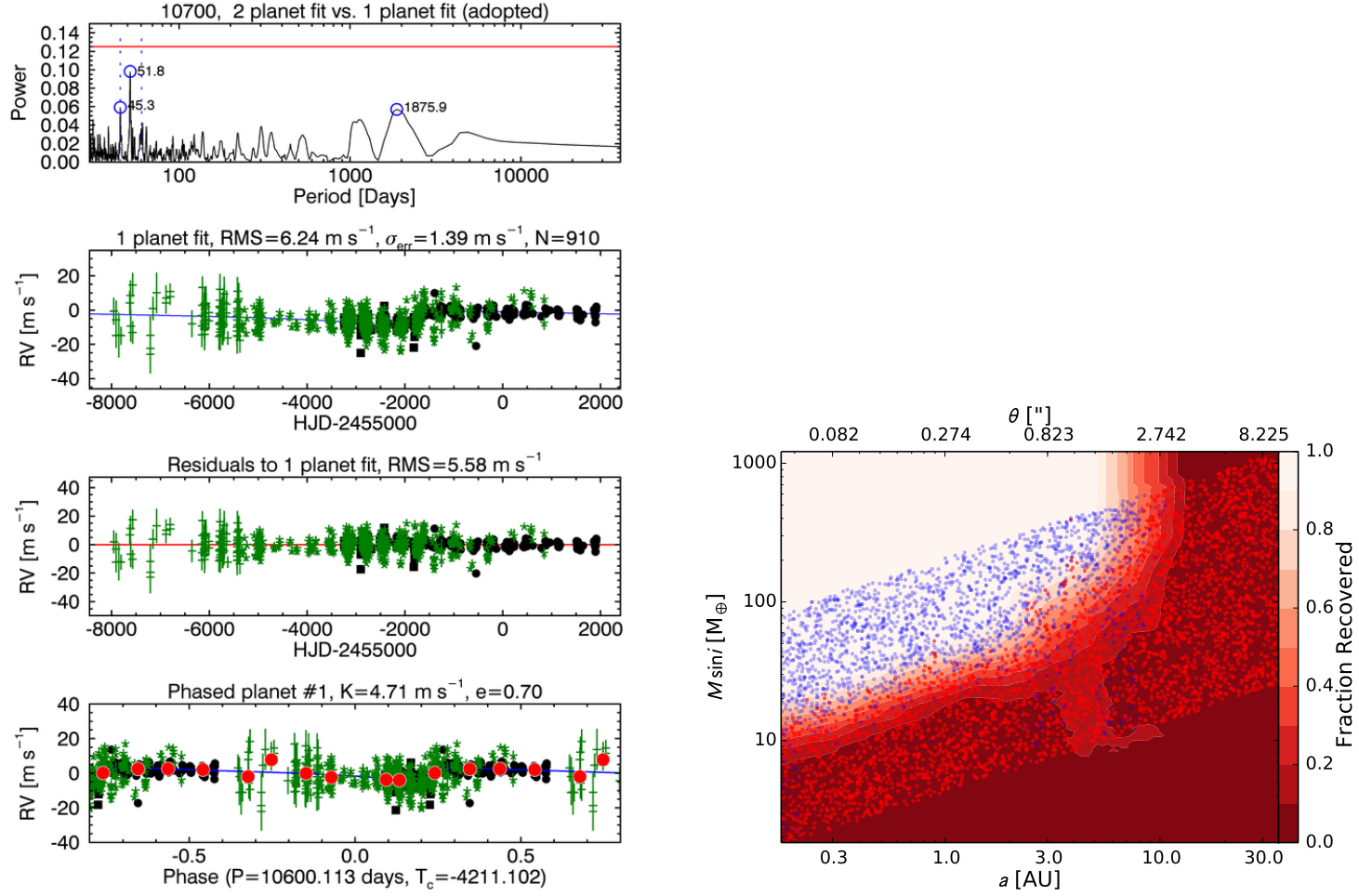

Fig. C.7.- Results from an automated search for planets orbiting the star HD 10700 (HIP 8102; programs = S, C, A) based on RVs from Lick and/or Keck Observatory. The set of plots on the left (analogous to Figures 4 and 5) show the planet search results and the plot on the right shows the completeness limits (analogous to Fig. 6). See the captions of those figures for detailed descriptions. The automated pipeline detects a long-period signal but it appears to be caused by a poorly constrained offset between datasets. We do not detect to five controversial, low-mass planets claimed by Tuomi et al. (2013). 

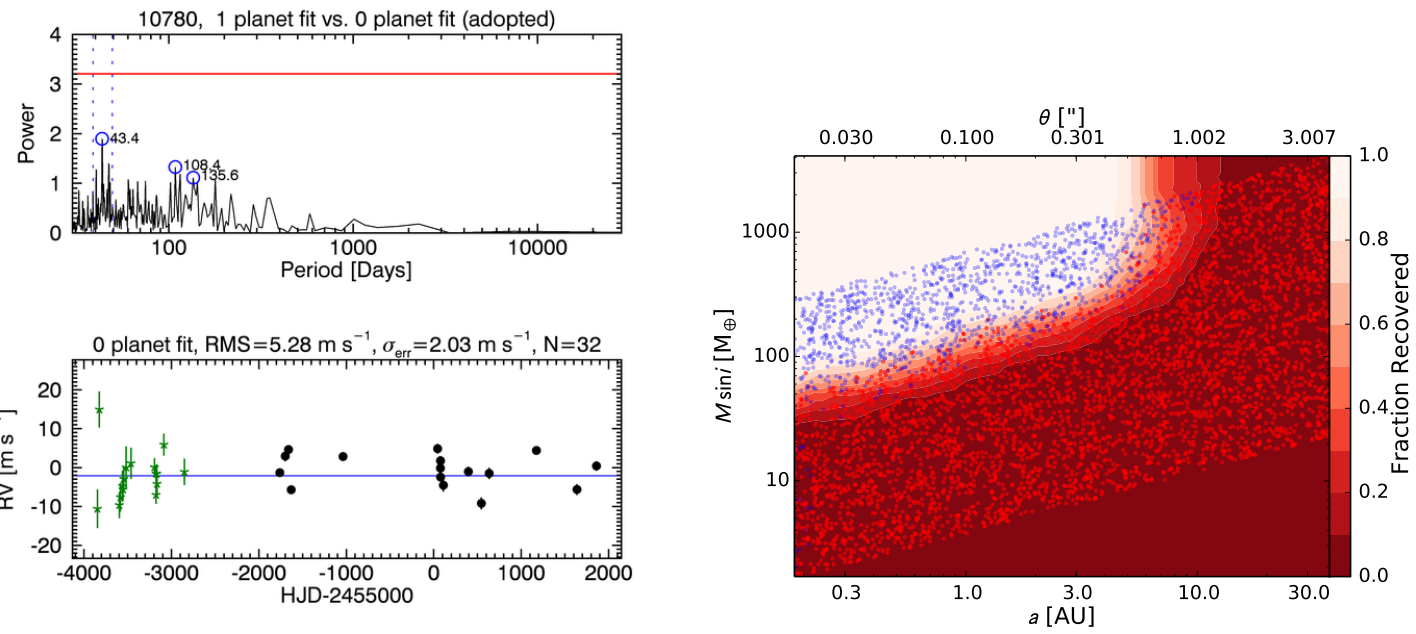

Fig. C.8.- Results from an automated search for planets orbiting the star HD 10780 (HIP 8362; program $=\mathrm{S}$ ) based on RVs from Lick and/or Keck Observatory. The set of plots on the left (analogous to Figures 4 and 5) show the planet search results and the plot on the right shows the completeness limits (analogous to Fig. 6). See the captions of those figures for detailed descriptions. 

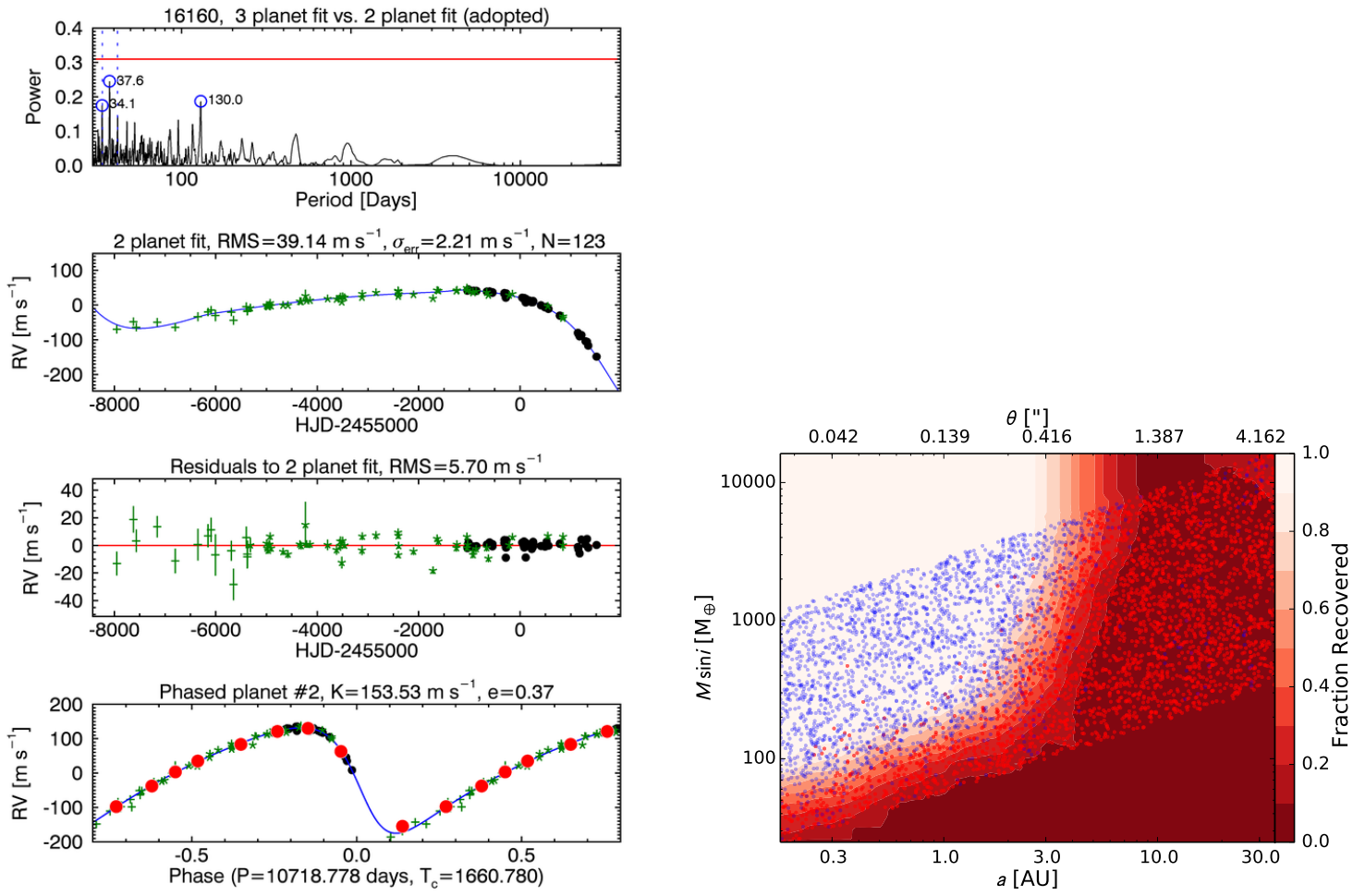

Fig. C.9.- Results from an automated search for planets orbiting the star HD 16160 (HIP 12114; program $=\mathrm{S}$ ) based on RVs from Lick and/or Keck Observatory. The set of plots on the left (analogous to Figures 4 and 5) show the planet search results and the plot on the right shows the completeness limits (analogous to Fig. 6). See the captions of those figures for detailed descriptions. The RV time-series for this star shows significant curvature from a late M-type companion. The RV curvature makes it difficult to detect long-period planets in this system. 

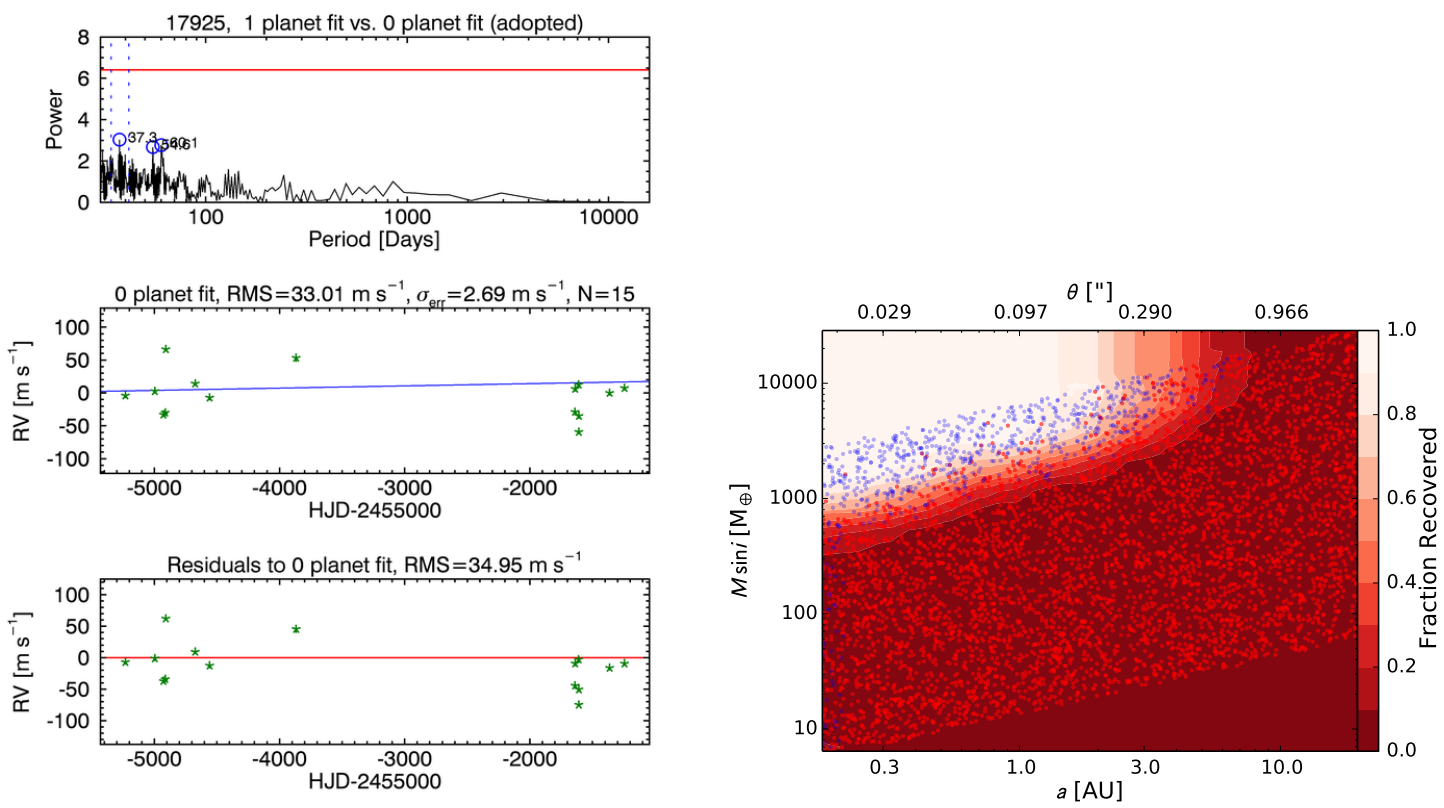

Fig. C.10.- Results from an automated search for planets orbiting the star HD 17925 $($ HIP 13402; program $=$ S) based on RVs from Lick and/or Keck Observatory. The set of plots on the left (analogous to Figures 4 and 5 ) show the planet search results and the plot on the right shows the completeness limits (analogous to Fig. 6). See the captions of those figures for detailed descriptions. The automated pipeline detects a marginally significant linear trend in the RV time series. 

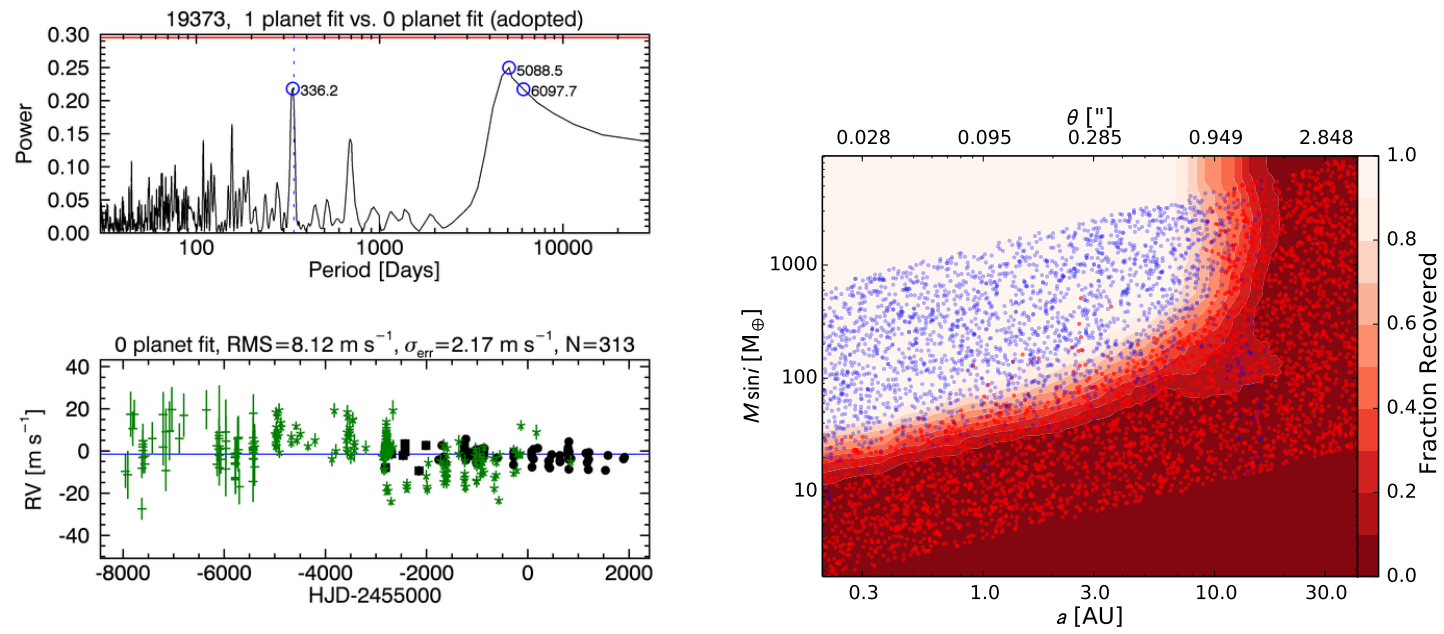

Fig. C.11. - Results from an automated search for planets orbiting the star HD 19373 (HIP 14632; programs = S, C, A) based on RVs from Lick and/or Keck Observatory. The set of plots on the left (analogous to Figures 4 and 5 show the planet search results and the plot on the right shows the completeness limits (analogous to Fig. 6). See the captions of those figures for detailed descriptions. 

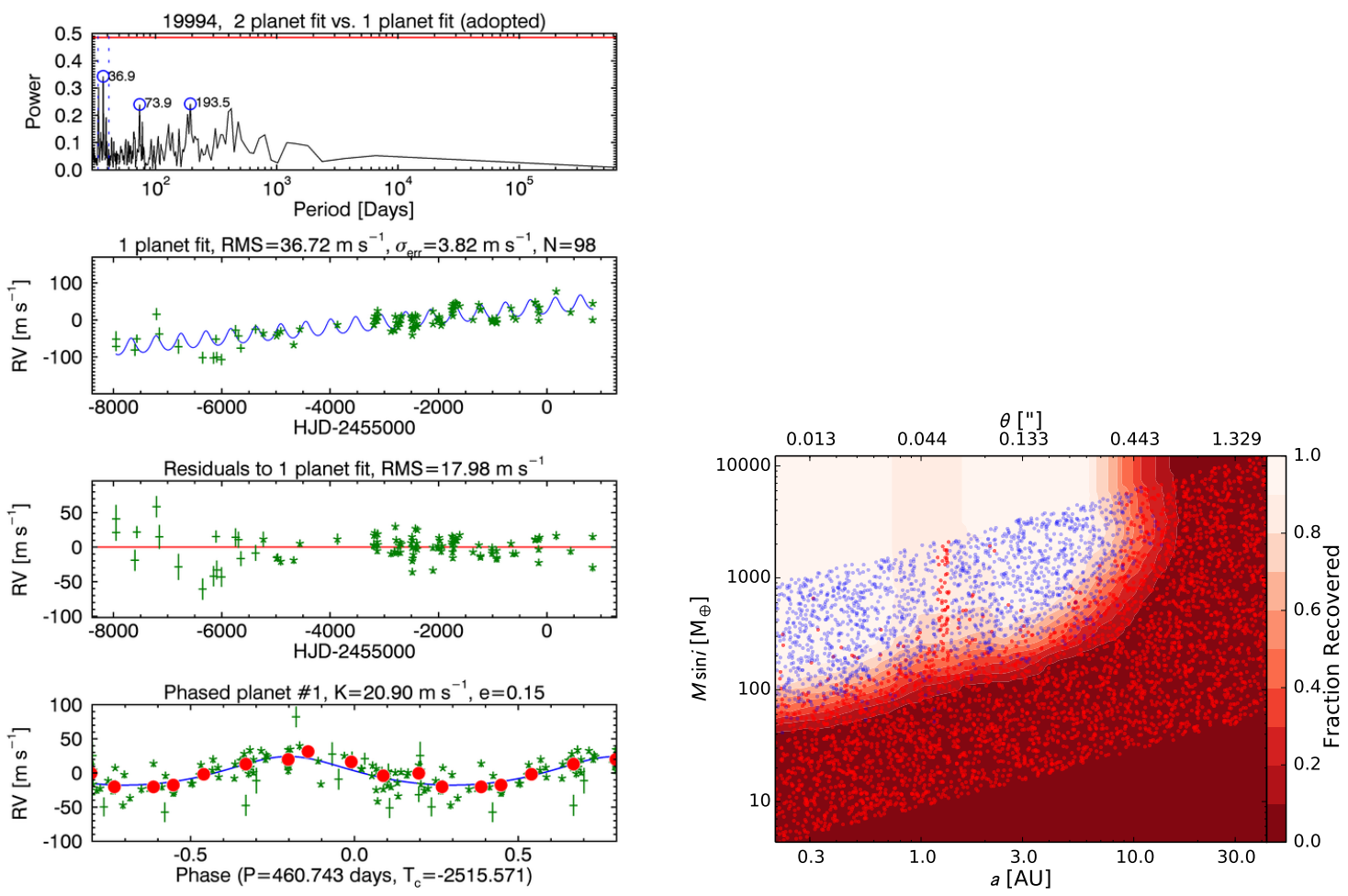

Fig. C.12.- Results from an automated search for planets orbiting the star HD 19994 (HIP 14954; program = A) based on RVs from Lick and/or Keck Observatory. The set of plots on the left (analogous to Figures 4 and 5 show the planet search results and the plot on the right shows the completeness limits (analogous to Fig. 6). See the captions of those figures for detailed descriptions. This star has one known planet. The pipeline also detects a linear trend in the RV time series. 

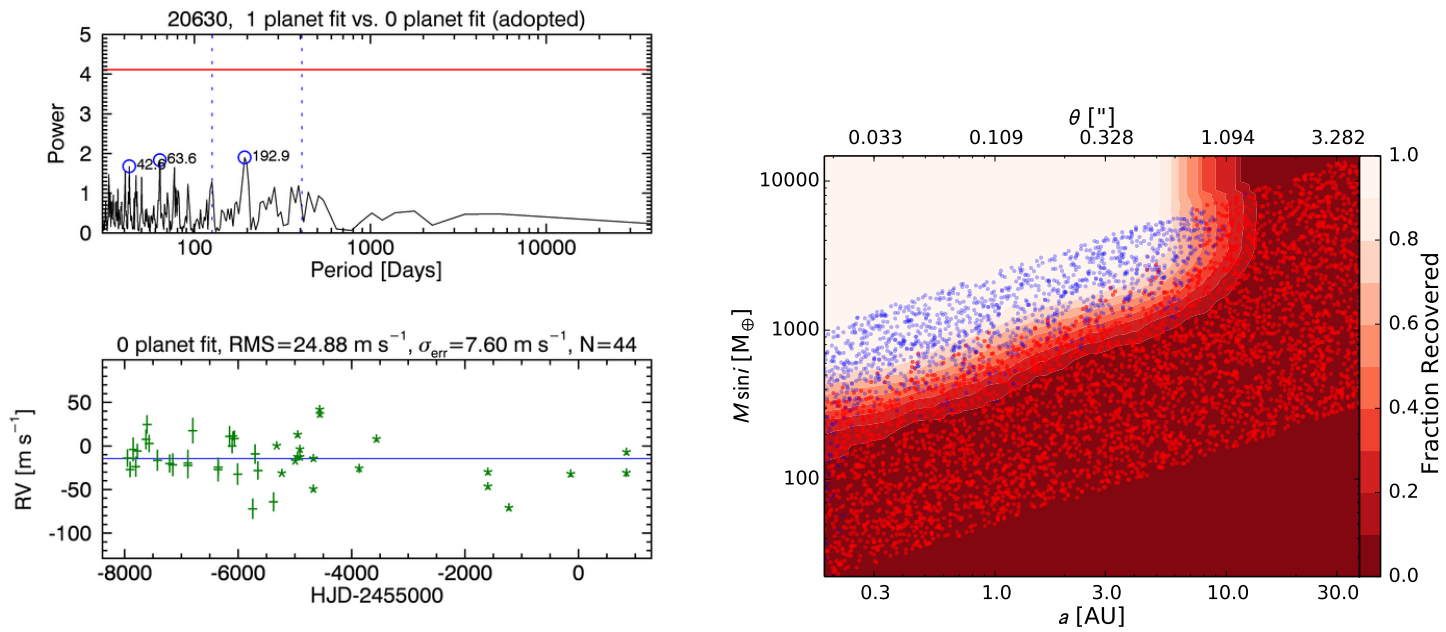

Fig. C.13.- Results from an automated search for planets orbiting the star HD 20630 (HIP 15457; programs = S, C, A) based on RVs from Lick and/or Keck Observatory. The set of plots on the left (analogous to Figures 4 and 5 show the planet search results and the plot on the right shows the completeness limits (analogous to Fig. 6). See the captions of those figures for detailed descriptions. 

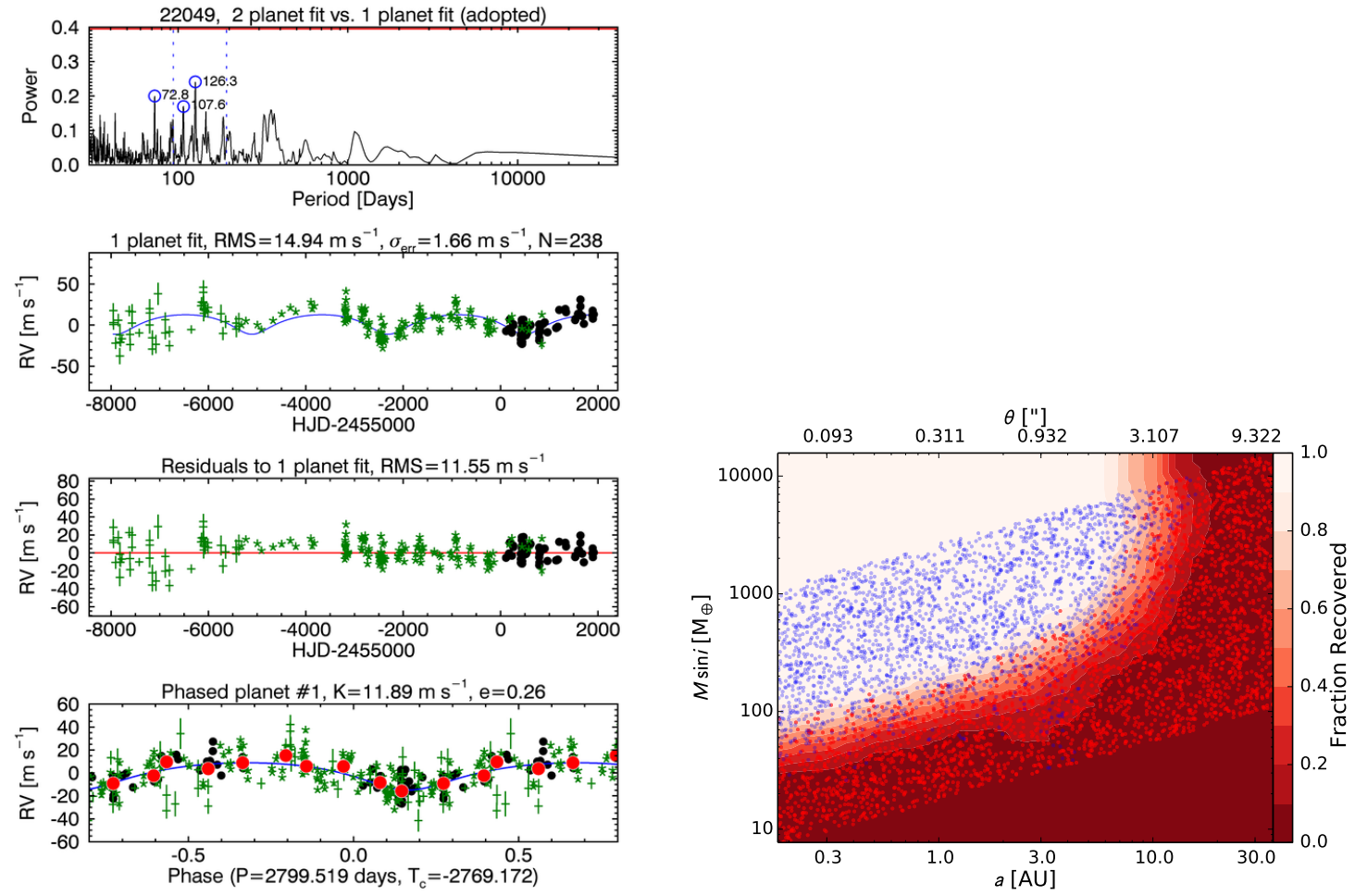

Fig. C.14. - Results from an automated search for planets orbiting the star HD 22049 (HIP 16537; programs = C, A) based on RVs from Lick and/or Keck Observatory. The set of plots on the left (analogous to Figures 4 and 5) show the planet search results and the plot on the right shows the completeness limits (analogous to Fig. 6). See the captions of those figures for detailed descriptions. This star has one known, long-period planet, which we clearly detect in the Rv time series. This claim has been controversial because the host star is young and active. However, our measurements of the activity-sensitive Ca II H \& $\mathrm{K}$ lines in Keck spectra do not correlate with the RVs. 

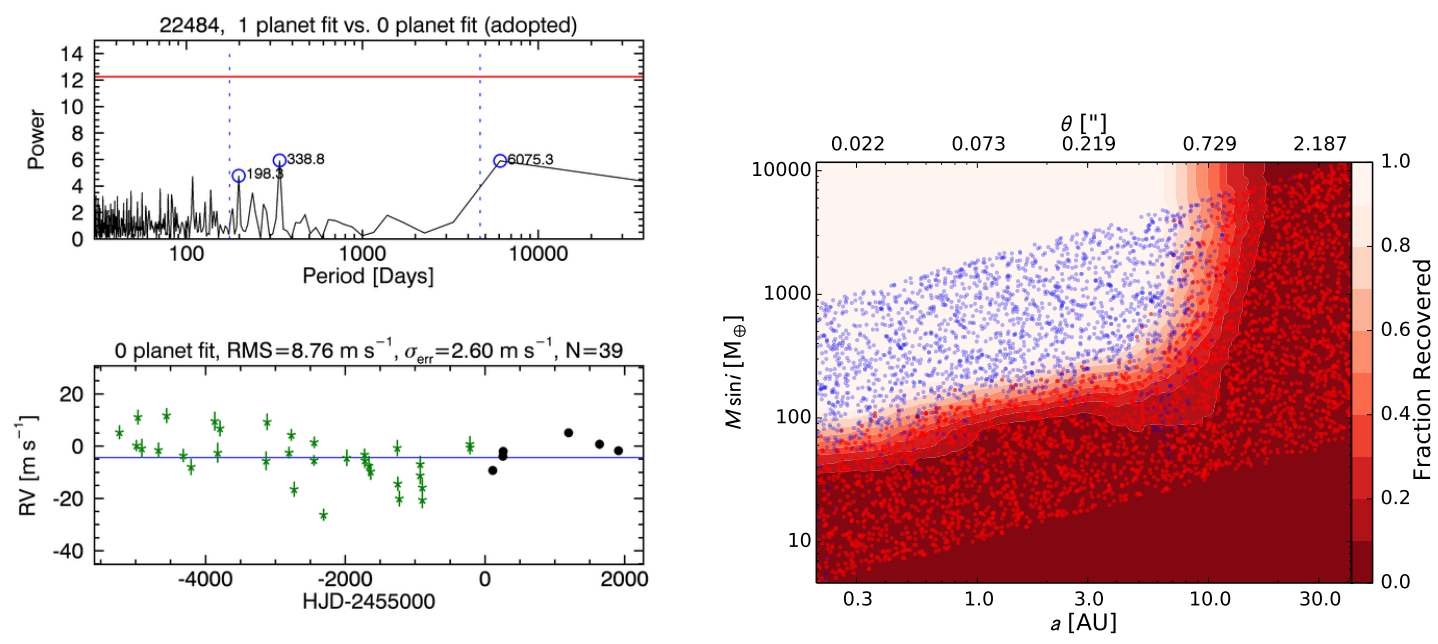

Fig. C.15. - Results from an automated search for planets orbiting the star HD 22484 (HIP 16852; programs = S, C, A) based on RVs from Lick and/or Keck Observatory. The set of plots on the left (analogous to Figures 4 and 5 ) show the planet search results and the plot on the right shows the completeness limits (analogous to Fig. 6). See the captions of those figures for detailed descriptions.
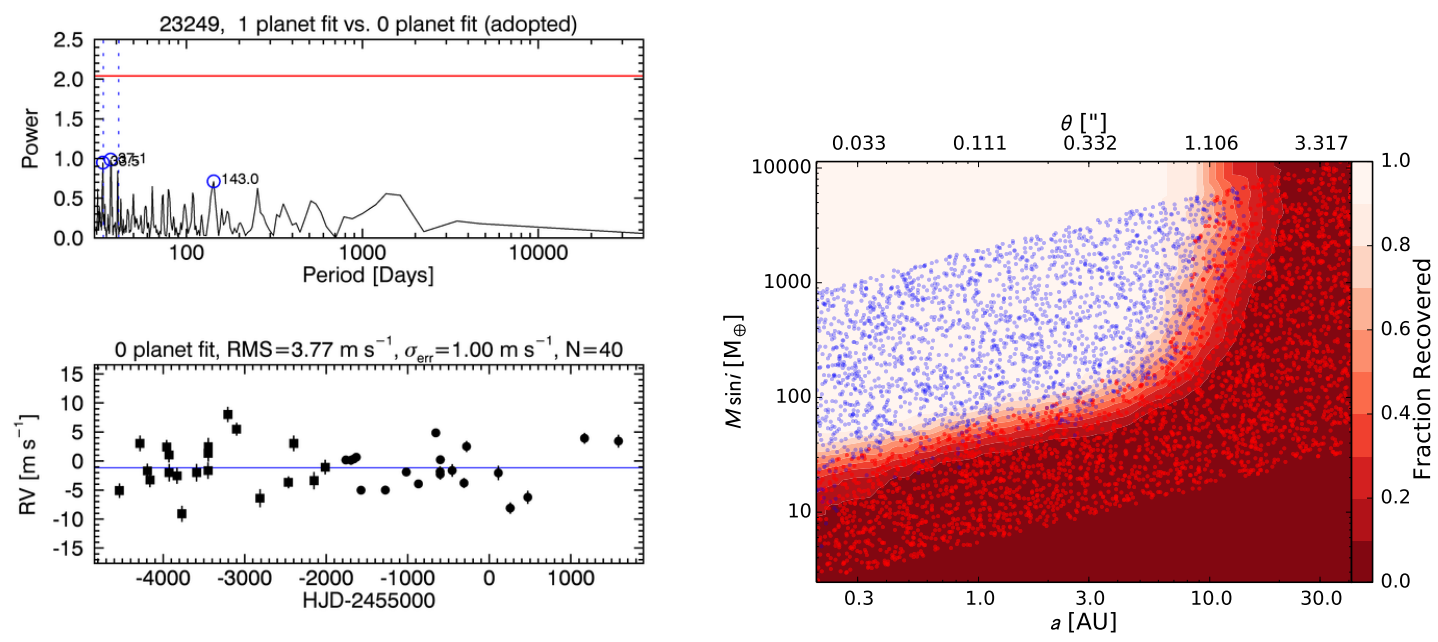

Fig. C.16. - Results from an automated search for planets orbiting the star HD 23249 (HIP 17378; programs = S, C) based on RVs from Lick and/or Keck Observatory. The set of plots on the left (analogous to Figures 4 and 5 show the planet search results and the plot on the right shows the completeness limits (analogous to Fig. 6). See the captions of those figures for detailed descriptions. 

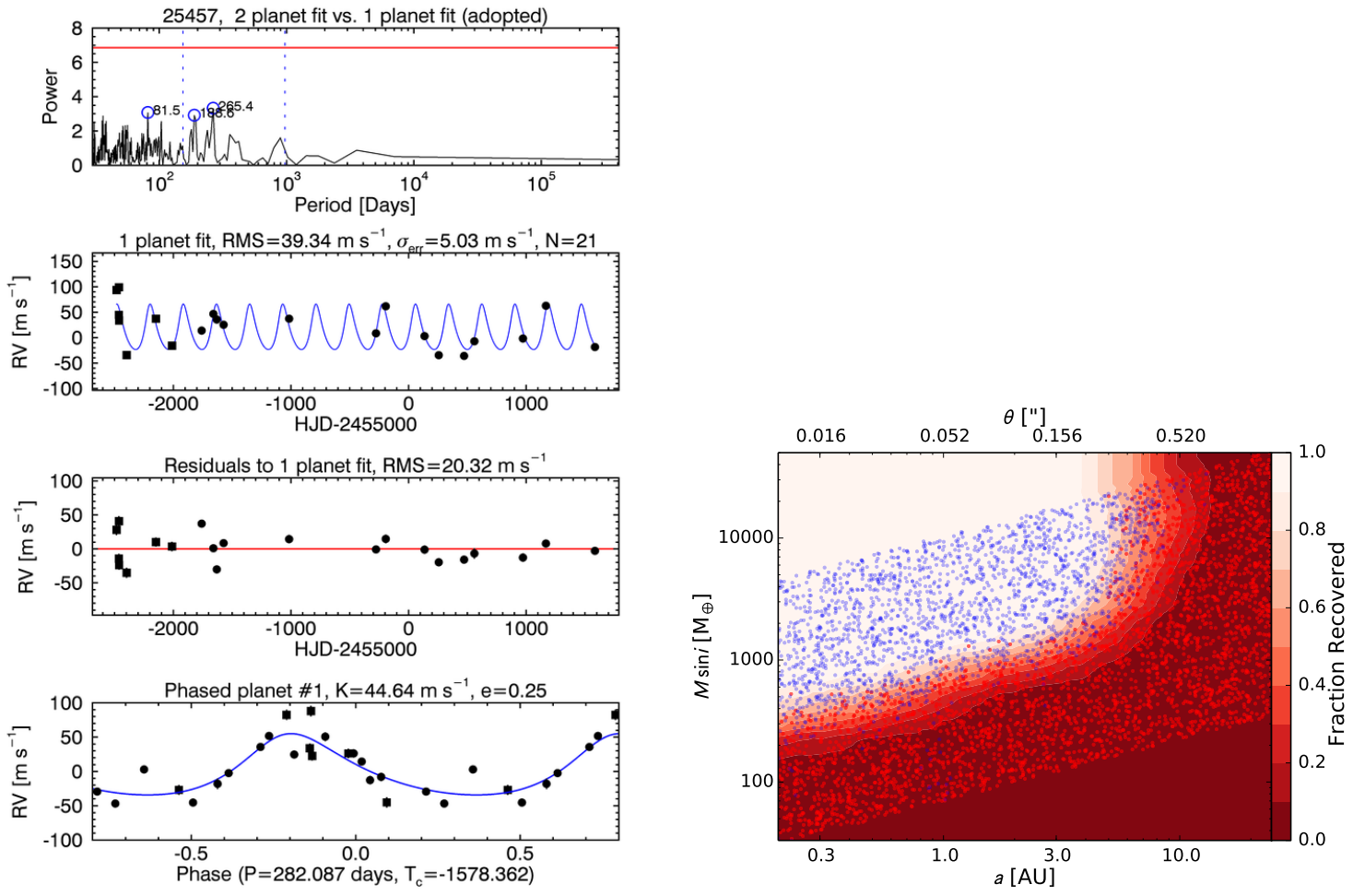

Fig. C.17.- Results from an automated search for planets orbiting the star HD 25457 (HIP 18859; program $=$ A) based on RVs from Lick and/or Keck Observatory. The set of plots on the left (analogous to Figures 4 and 5) show the planet search results and the plot on the right shows the completeness limits (analogous to Fig. 6). See the captions of those figures for detailed descriptions. The automated pipeline formally identifies a planet candidate based on 21 Keck RVs. Given the high jitter and small number of observations, we deem this candidate not credible with the current data. 

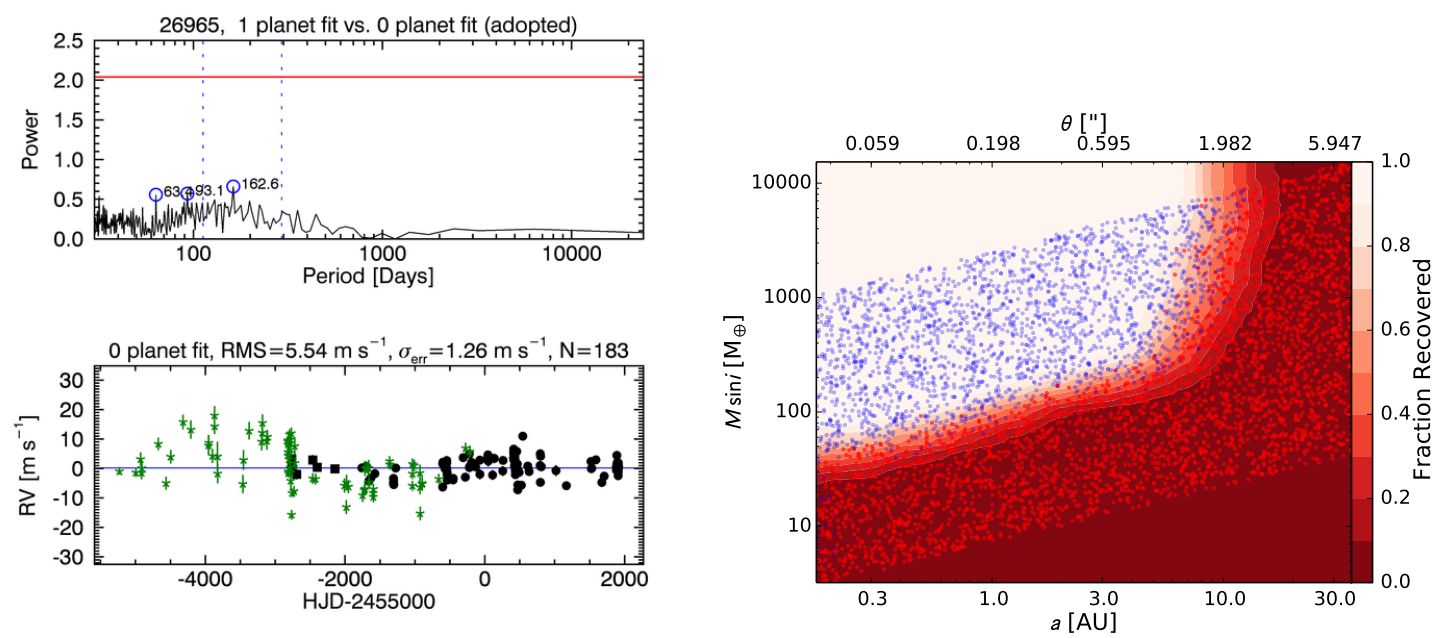

Fig. C.18. - Results from an automated search for planets orbiting the star HD 26965 (HIP 19849; programs = S, C, A) based on RVs from Lick and/or Keck Observatory. The set of plots on the left (analogous to Figures 4 and 5 show the planet search results and the plot on the right shows the completeness limits (analogous to Fig. 6). See the captions of those figures for detailed descriptions. 

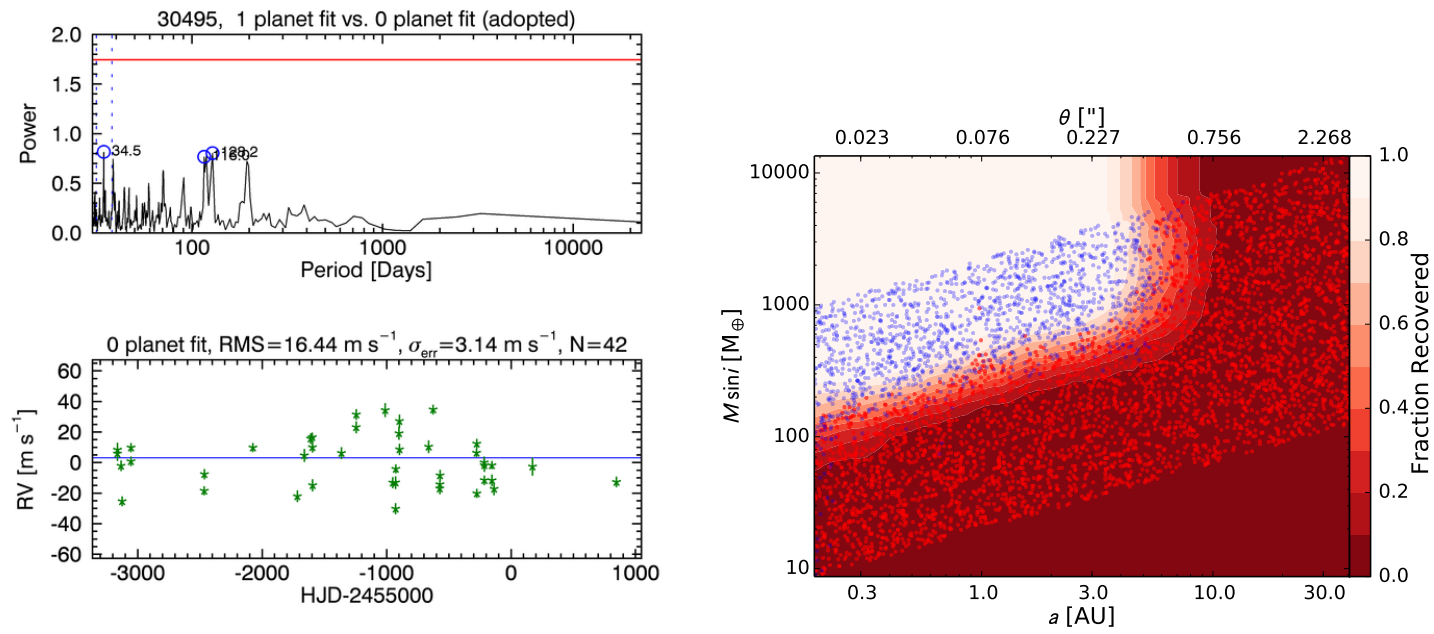

Fig. C.19. - Results from an automated search for planets orbiting the star HD 30495 (HIP 22263; program = S) based on RVs from Lick and/or Keck Observatory. The set of plots on the left (analogous to Figures 4 and 5) show the planet search results and the plot on the right shows the completeness limits (analogous to Fig. 6). See the captions of those figures for detailed descriptions. 

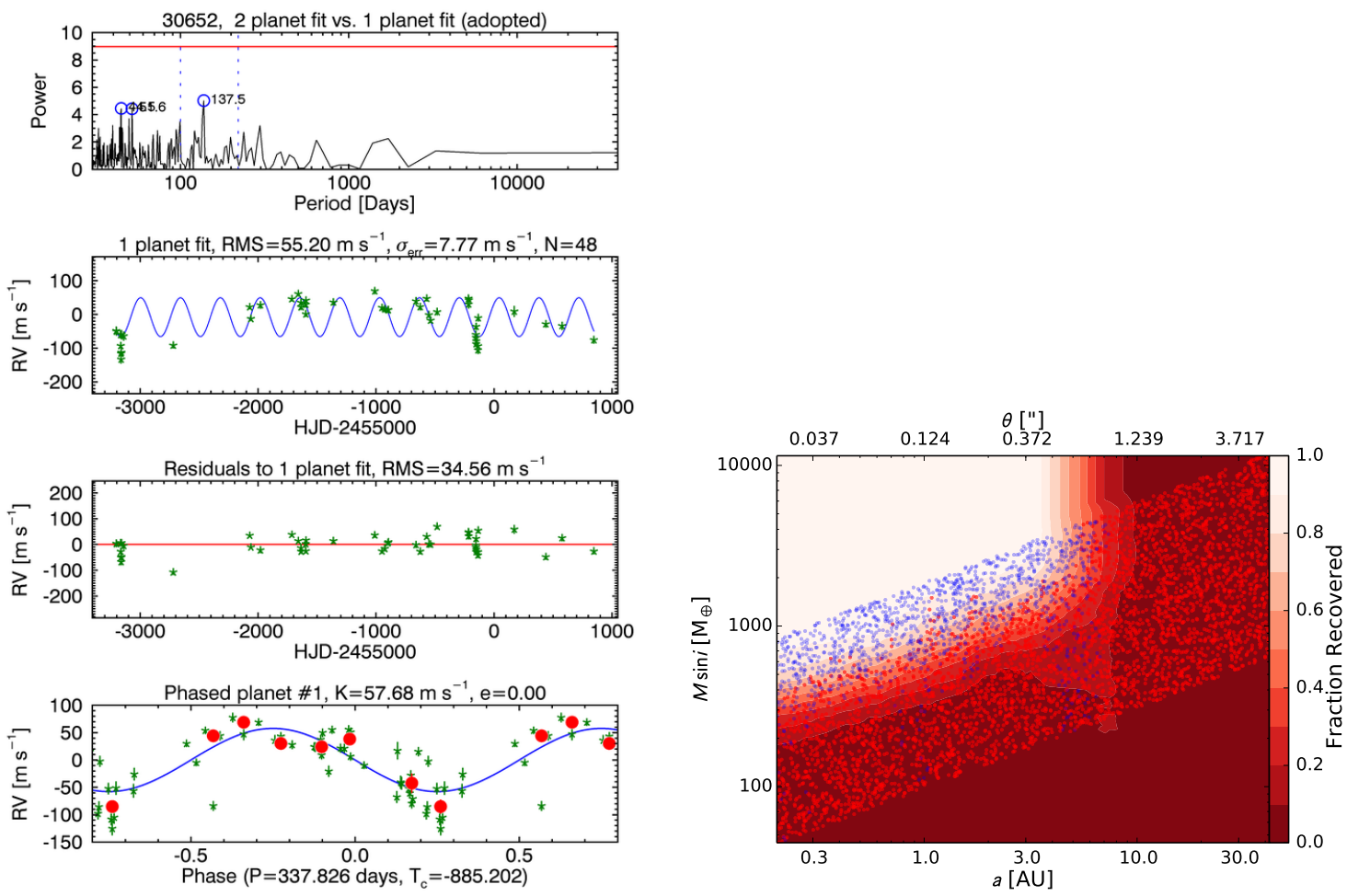

Fig. C.20.- Results from an automated search for planets orbiting the star HD 30652 (HIP 22449; programs = S, C, A) based on RVs from Lick and/or Keck Observatory. The set of plots on the left (analogous to Figures 4 and 5 show the planet search results and the plot on the right shows the completeness limits (analogous to Fig. 6). See the captions of those figures for detailed descriptions. We detect a marginally significant periodic signal with a period of 338 days. However, the poor observing history and the proximity of the period to one year, we conclude that this signal is not caused by a real planetary companion. The nature of the signal is most likely non-astrophysical in nature. 

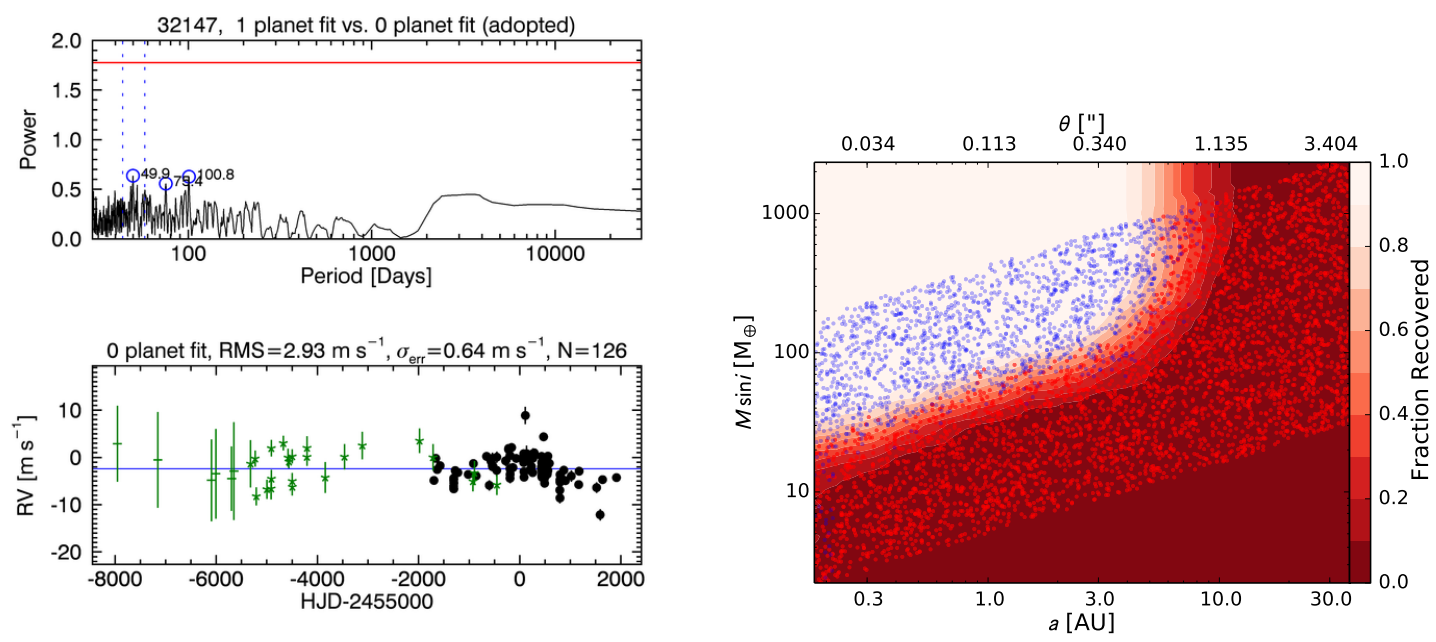

Fig. C.21. - Results from an automated search for planets orbiting the star HD 32147 (HIP 23311; program $=$ S) based on RVs from Lick and/or Keck Observatory. The set of plots on the left (analogous to Figures 4 and 5) show the planet search results and the plot on the right shows the completeness limits (analogous to Fig. 6). See the captions of those figures for detailed descriptions.
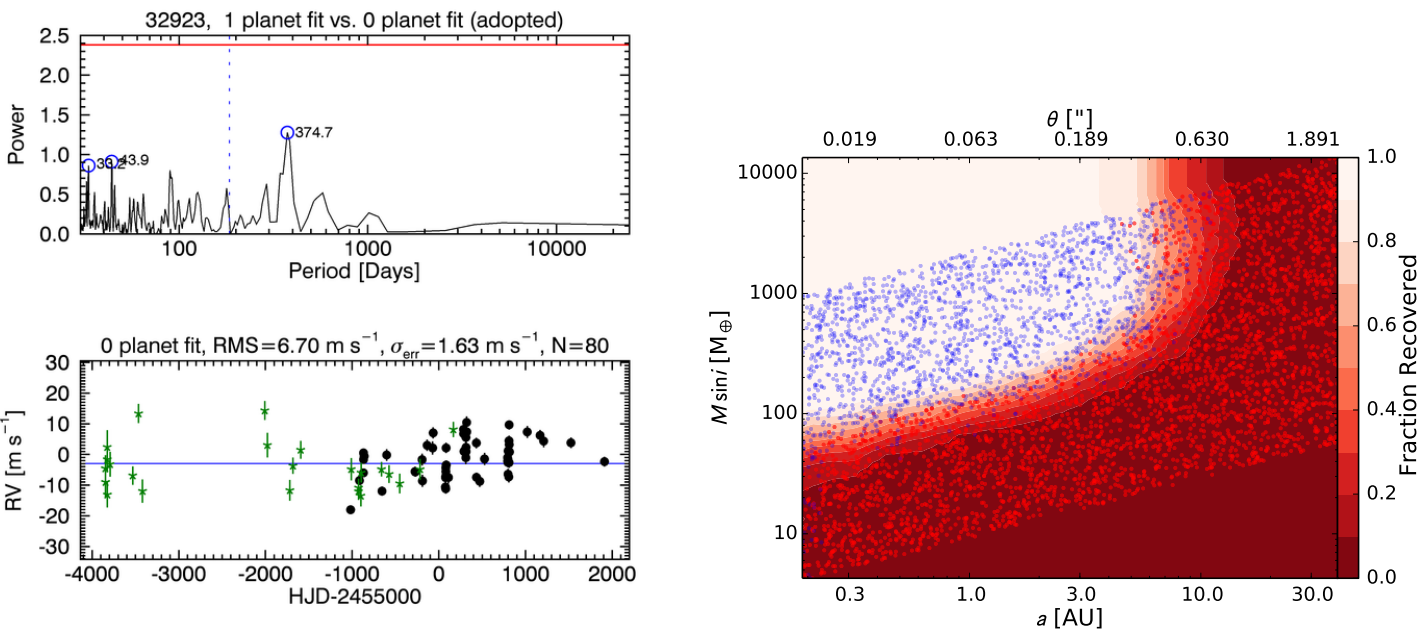

Fig. C.22. - Results from an automated search for planets orbiting the star HD 32923 (HIP 23835; programs = S, C, A) based on RVs from Lick and/or Keck Observatory. The set of plots on the left (analogous to Figures 4 and 5 show the planet search results and the plot on the right shows the completeness limits (analogous to Fig. 6). See the captions of those figures for detailed descriptions. 

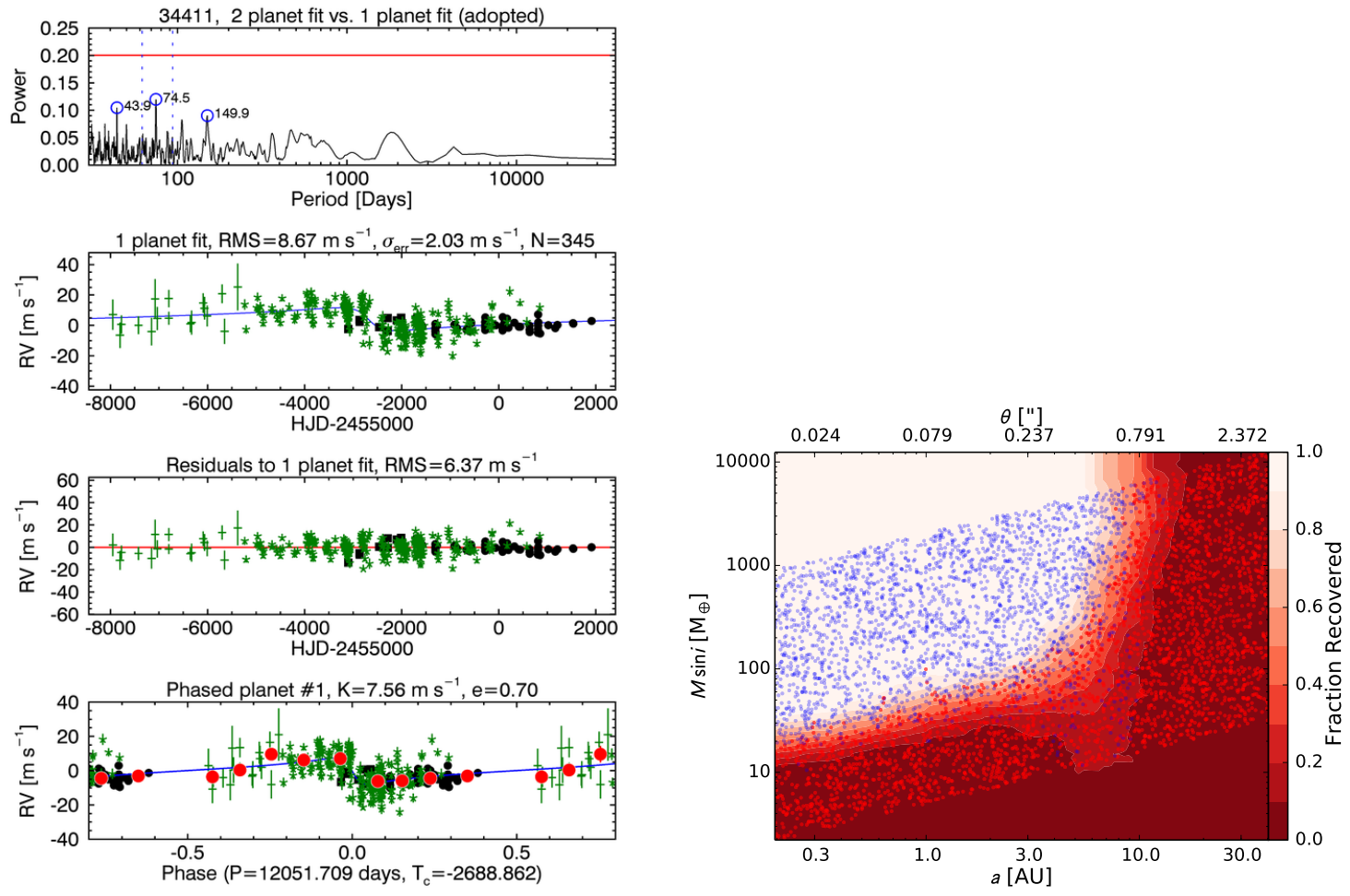

Fig. C.23. - Results from an automated search for planets orbiting the star HD 34411 (HIP 24813; programs = S, C, A) based on RVs from Lick and/or Keck Observatory. The set of plots on the left (analogous to Figures 4 and 5 ) show the planet search results and the plot on the right shows the completeness limits (analogous to Fig. 6). See the captions of those figures for detailed descriptions. The automated pipeline picks up a long-period, high-eccentricity signal that is likely due to poorly constrained offsets between instruments. 

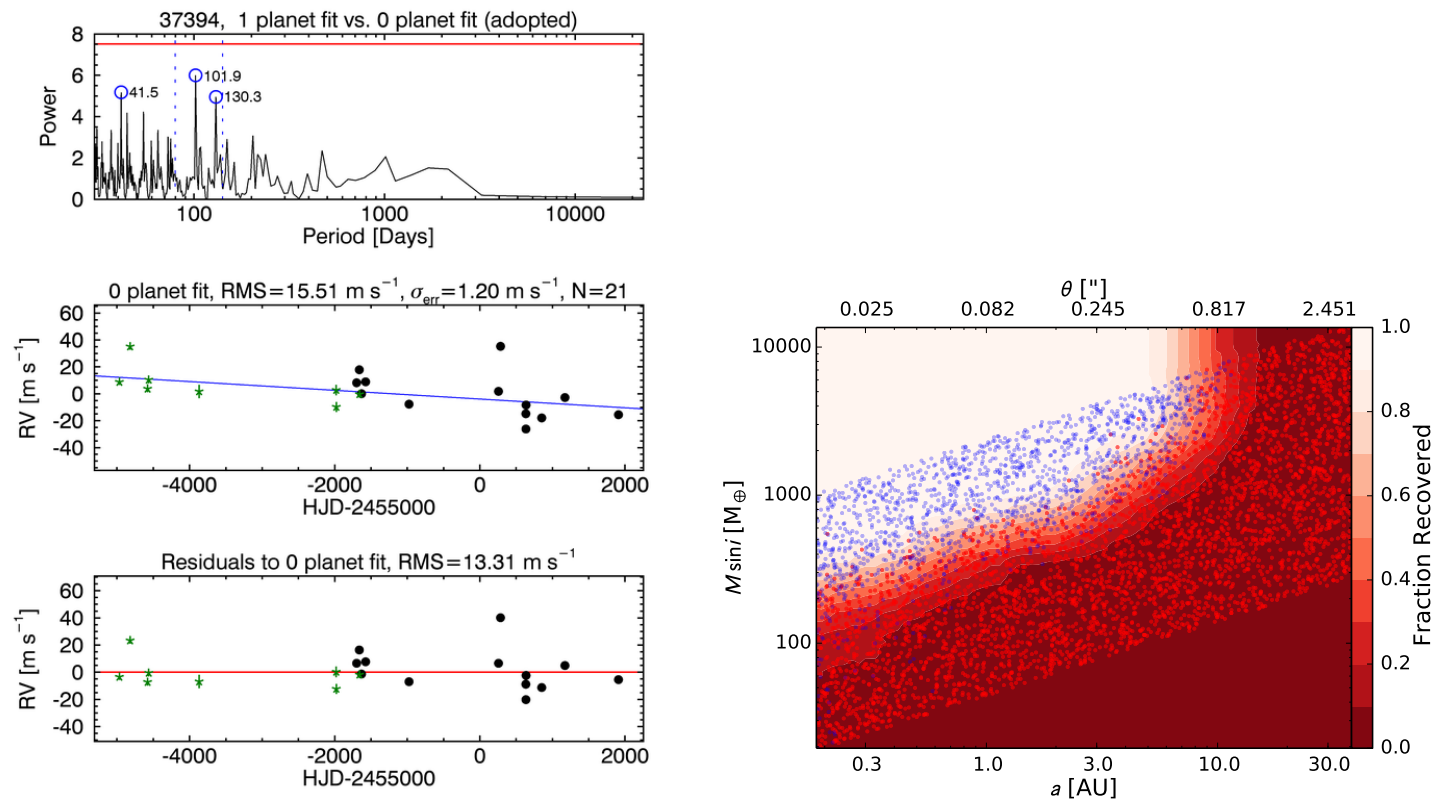

Fig. C.24. - Results from an automated search for planets orbiting the star HD 37394 (HIP 26779; program = S) based on RVs from Lick and/or Keck Observatory. The set of plots on the left (analogous to Figures 4 and 5 ) show the planet search results and the plot on the right shows the completeness limits (analogous to Fig. 6). See the captions of those figures for detailed descriptions. This young, active star shows a small, marginally significant linear trend. 

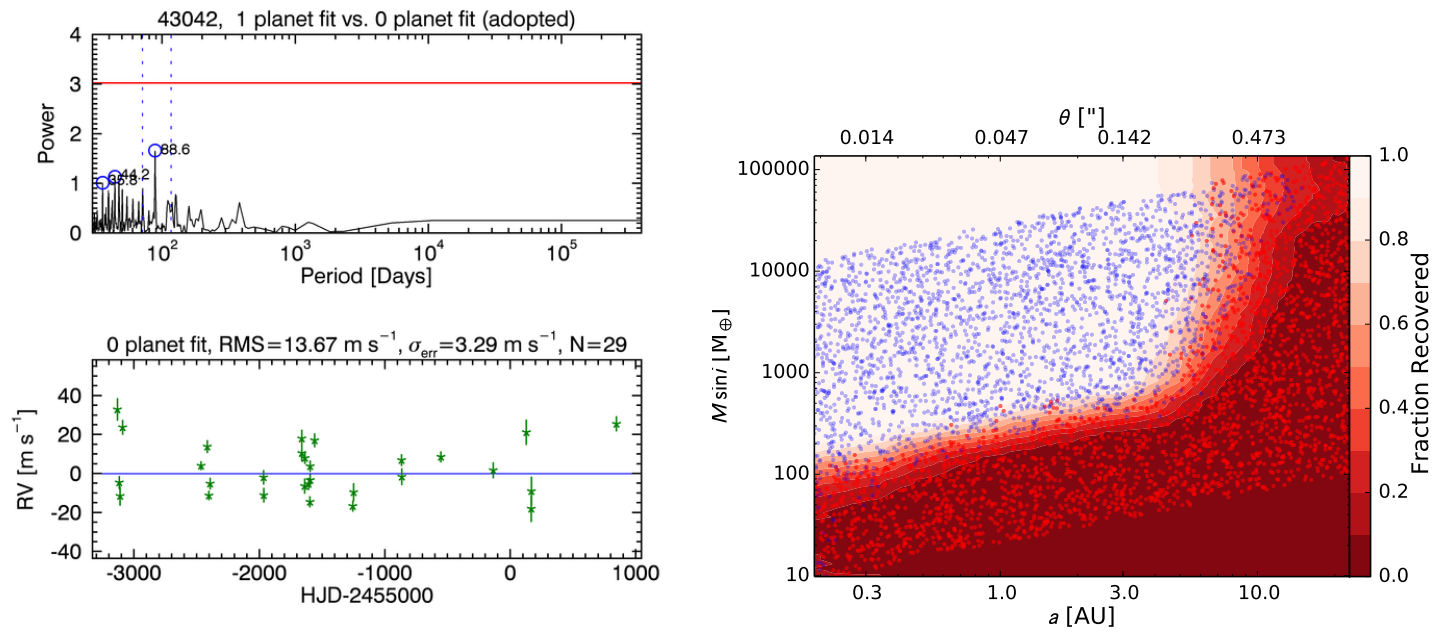

Fig. C.25. - Results from an automated search for planets orbiting the star HD 43042 (HIP 29650; program = A) based on RVs from Lick and/or Keck Observatory. The set of plots on the left (analogous to Figures 4 and 5) show the planet search results and the plot on the right shows the completeness limits (analogous to Fig. 6). See the captions of those figures for detailed descriptions. 

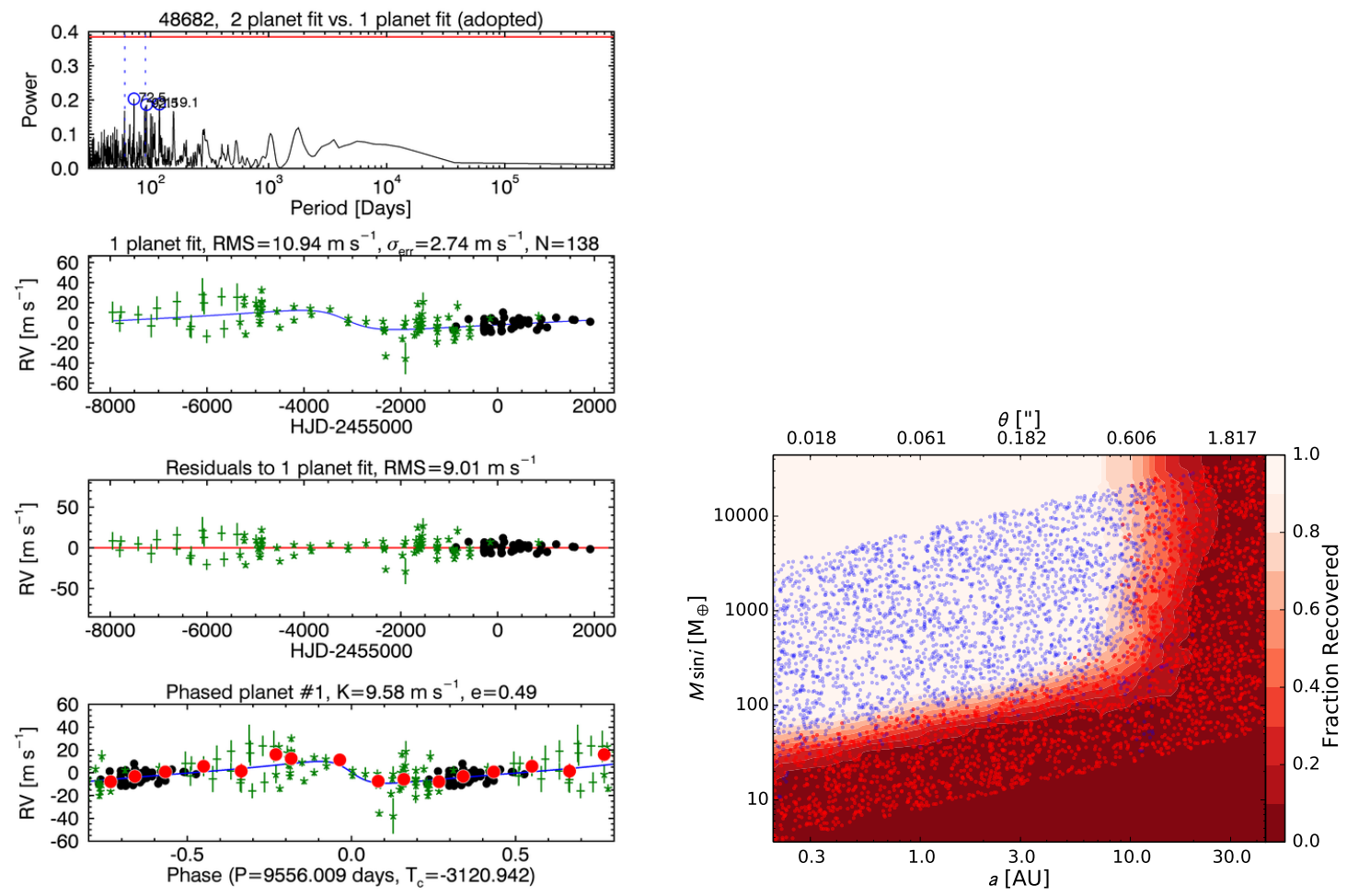

Fig. C.26. - Results from an automated search for planets orbiting the star HD 48682 (HIP 32480; program = A) based on RVs from Lick and/or Keck Observatory. The set of plots on the left (analogous to Figures 4 and 5) show the planet search results and the plot on the right shows the completeness limits (analogous to Fig. 6). See the captions of those figures for detailed descriptions. This star has a formally adopted signal that appears to be due to uncorrected zero-point offsets in the Lick RVs and not due a planet. 

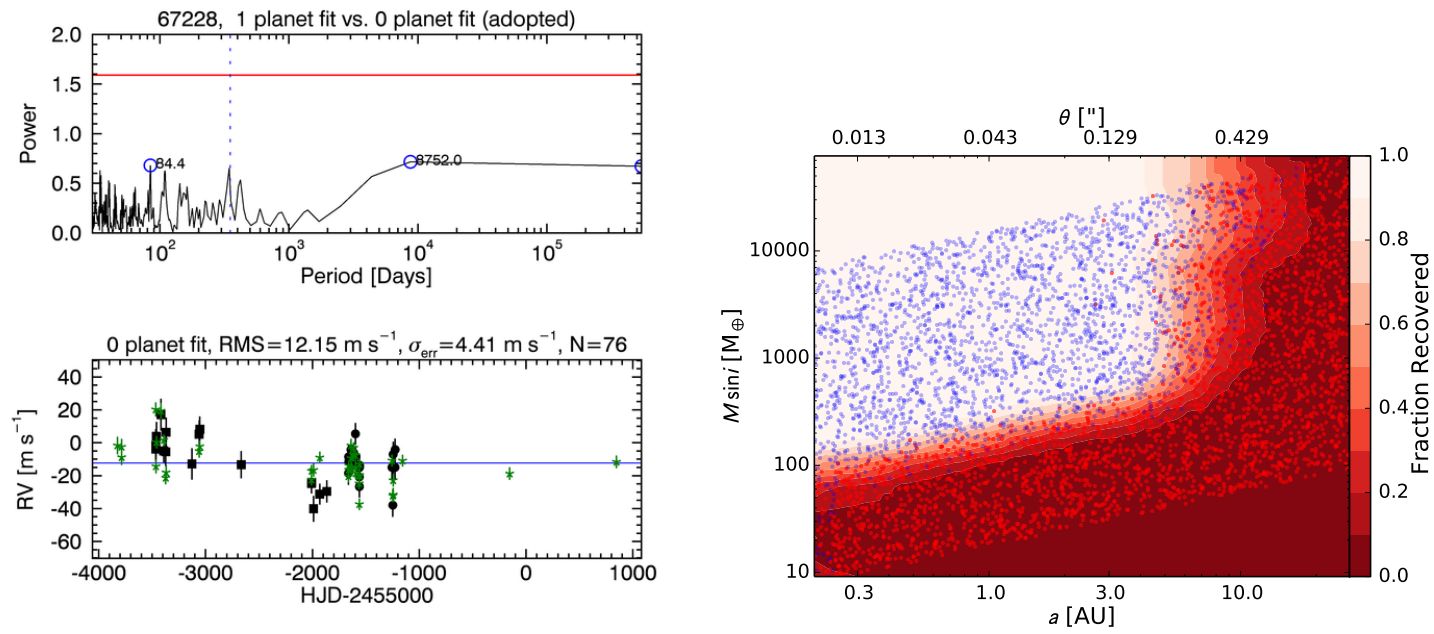

Fig. C.27. - Results from an automated search for planets orbiting the star HD 67228 (HIP 39780; program = A) based on RVs from Lick and/or Keck Observatory. The set of plots on the left (analogous to Figures 4 and 5) show the planet search results and the plot on the right shows the completeness limits (analogous to Fig. 6). See the captions of those figures for detailed descriptions. 

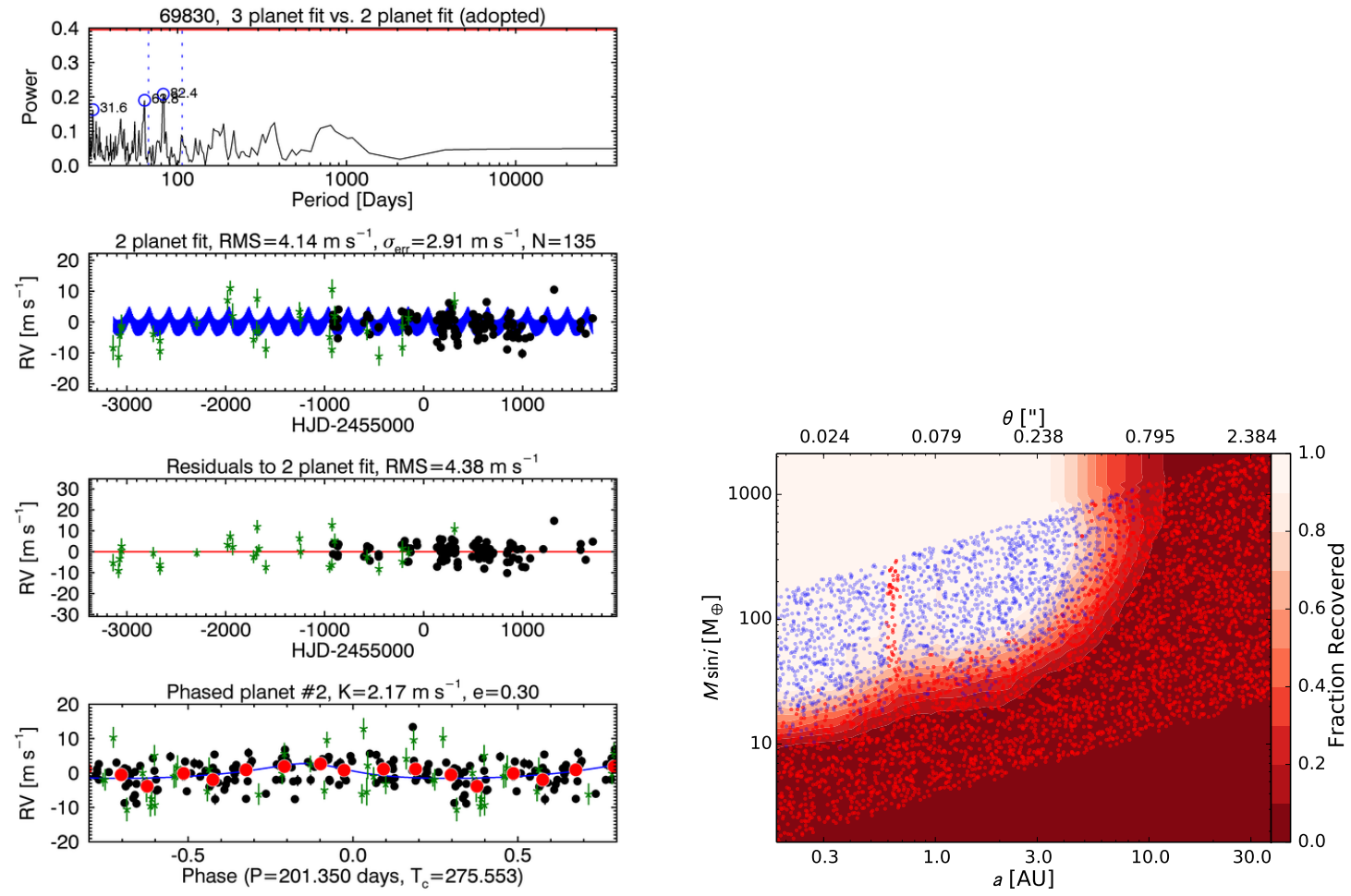

Fig. C.28. - Results from an automated search for planets orbiting the star HD 69830 (HIP 40693; programs = S) based on RVs from Lick and/or Keck Observatory. The set of plots on the left (analogous to Figures 4 and 5) show the planet search results and the plot on the right shows the completeness limits (analogous to Fig. 6). See the captions of those figures for detailed descriptions. This star hosts three Neptune-mass planets, two of which we detect with our Keck and Lick RVs. While we do not detect the 31.6 day signal, we have not performed an analysis to show that our non-detection is dispositive. 

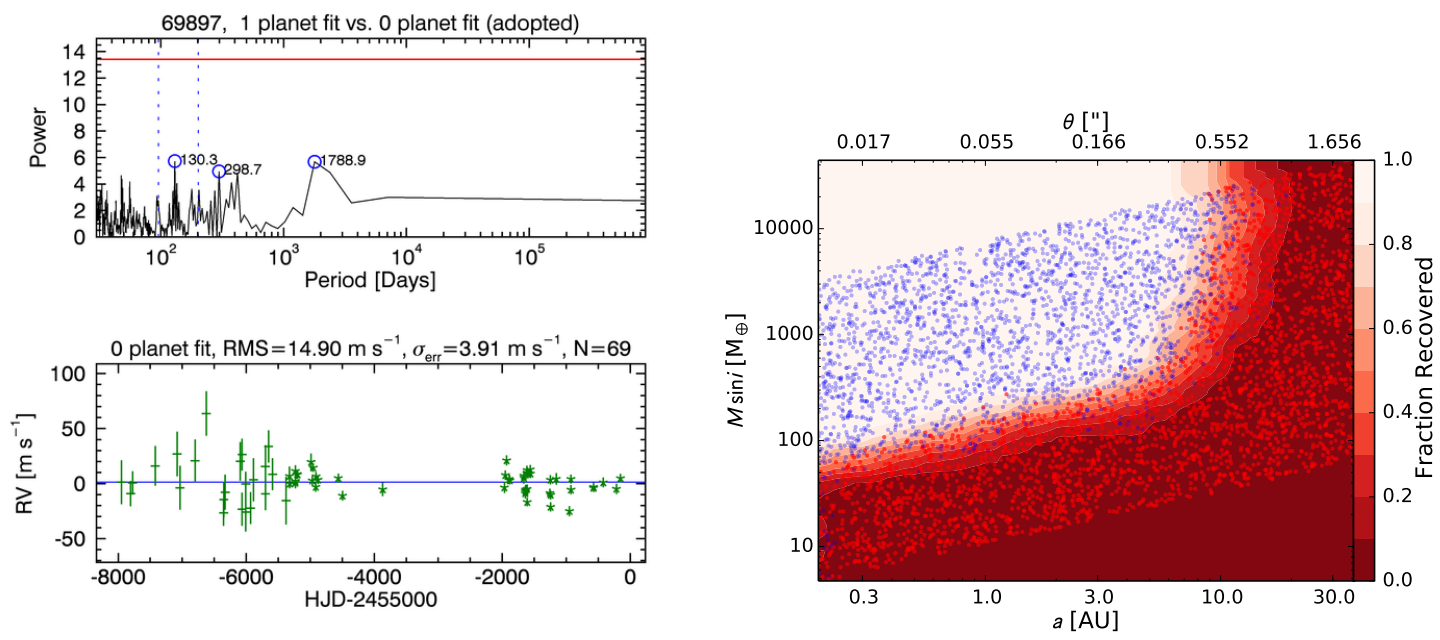

Fig. C.29. - Results from an automated search for planets orbiting the star HD 69897 (HIP 40843; program = A) based on RVs from Lick and/or Keck Observatory. The set of plots on the left (analogous to Figures 4 and 5) show the planet search results and the plot on the right shows the completeness limits (analogous to Fig. 6). See the captions of those figures for detailed descriptions.
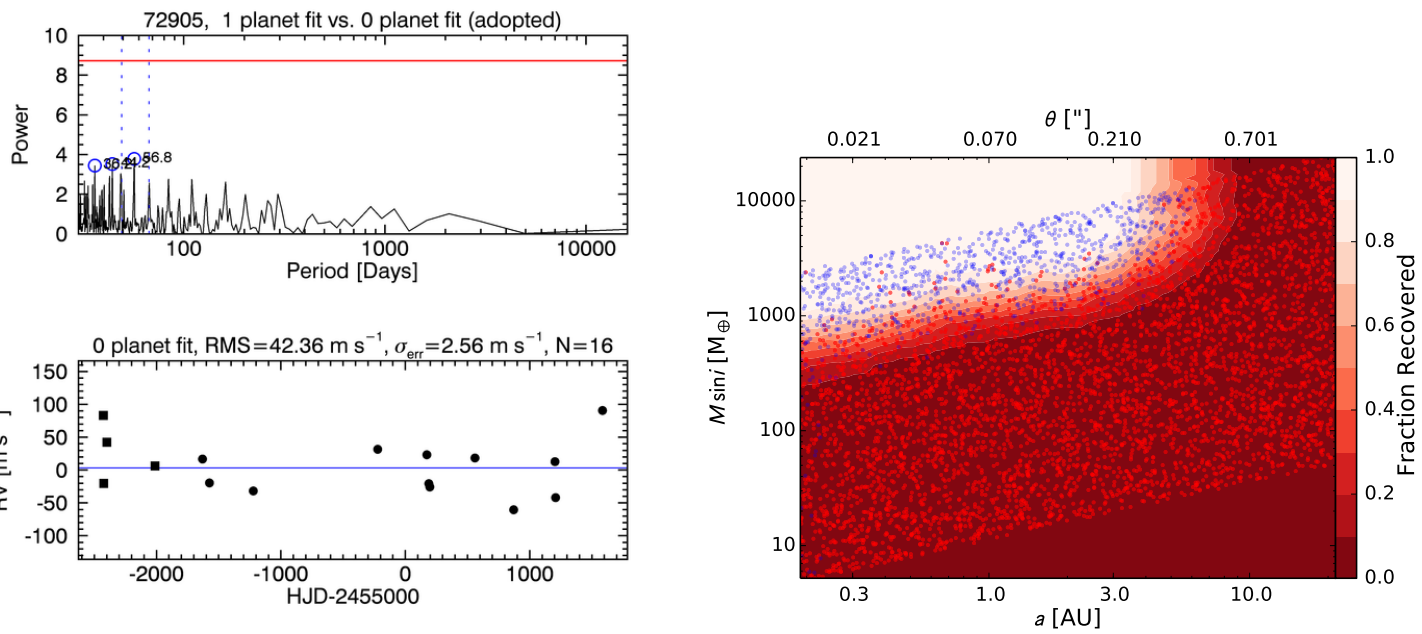

Fig. C.30.- Results from an automated search for planets orbiting the star HD 72905 (HIP 42438; program $=\mathrm{S}$ ) based on RVs from Lick and/or Keck Observatory. The set of plots on the left (analogous to Figures 4 and 5) show the planet search results and the plot on the right shows the completeness limits (analogous to Fig. 6). See the captions of those figures for detailed descriptions. 

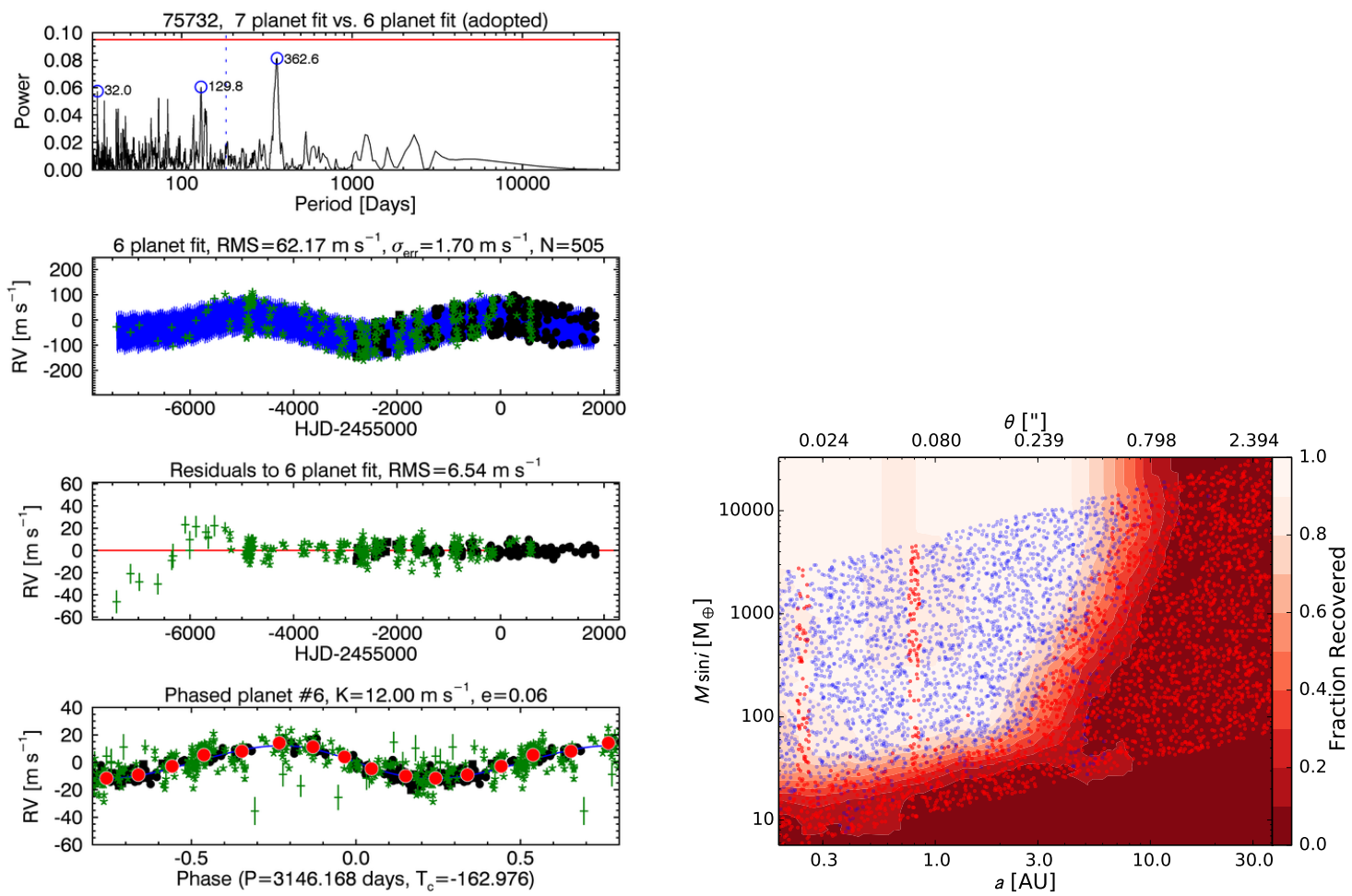

Fig. C.31. - Results from an automated search for planets orbiting the star HD 75732 (HIP 43587; program = S) based on RVs from Lick and/or Keck Observatory. The set of plots on the left (analogous to Figures 4 and 5) show the planet search results and the plot on the right shows the completeness limits (analogous to Fig. 6). See the captions of those figures for detailed descriptions. This star hosts five known planets. 

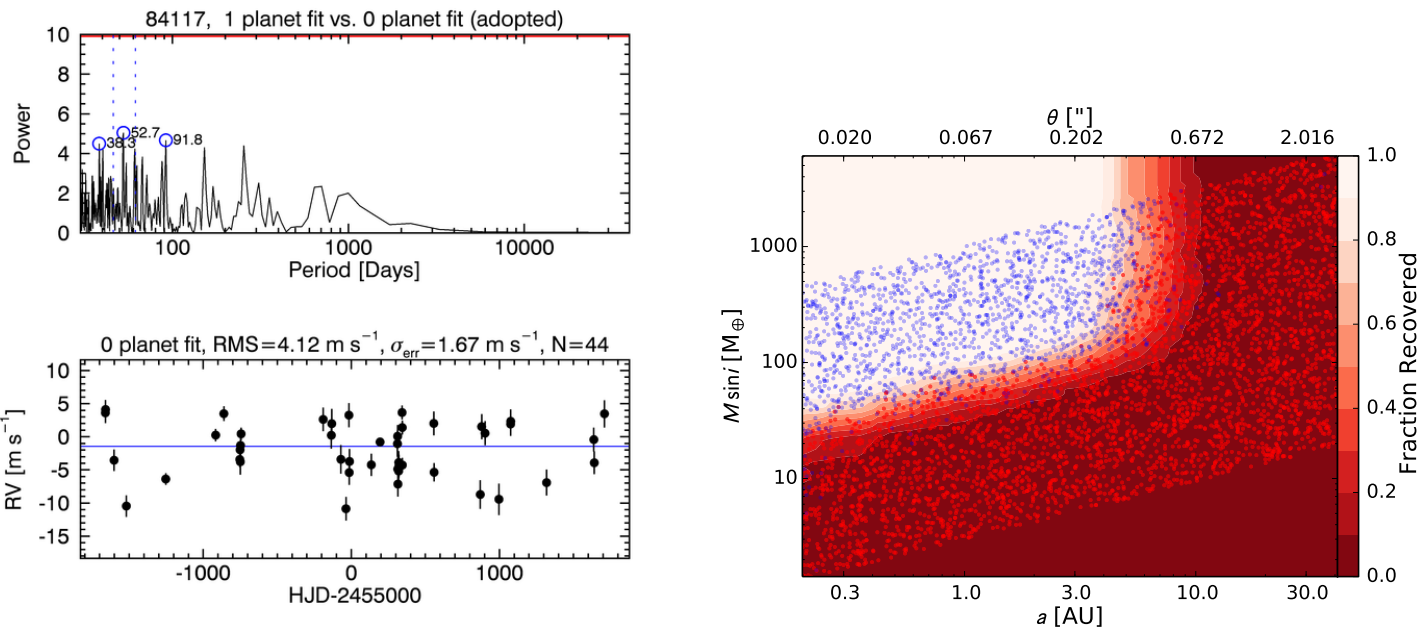

Fig. C.32. - Results from an automated search for planets orbiting the star HD 84117 (HIP 47592; programs = S, C, A) based on RVs from Lick and/or Keck Observatory. The set of plots on the left (analogous to Figures 4 and 5 show the planet search results and the plot on the right shows the completeness limits (analogous to Fig. 6). See the captions of those figures for detailed descriptions. 

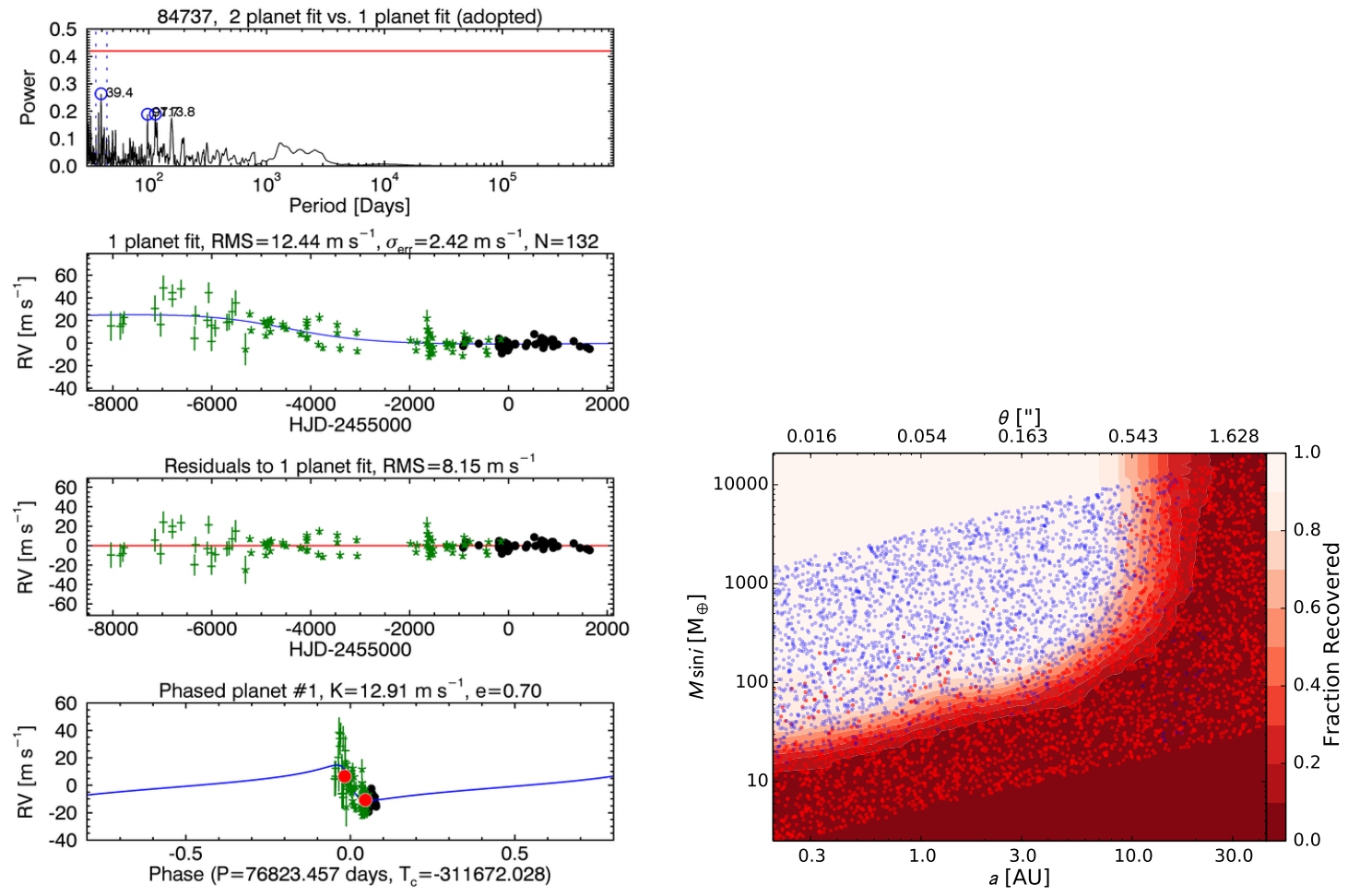

Fig. C.33. - Results from an automated search for planets orbiting the star HD 84737 (HIP 48113; program = A) based on RVs from Lick and/or Keck Observatory. The set of plots on the left (analogous to Figures 4 and 5) show the planet search results and the plot on the right shows the completeness limits (analogous to Fig. 6). See the captions of those figures for detailed descriptions. This star has a formally adopted signal that appears to be due to uncorrected zero-point offsets in the Lick RVs and not due a planet. 

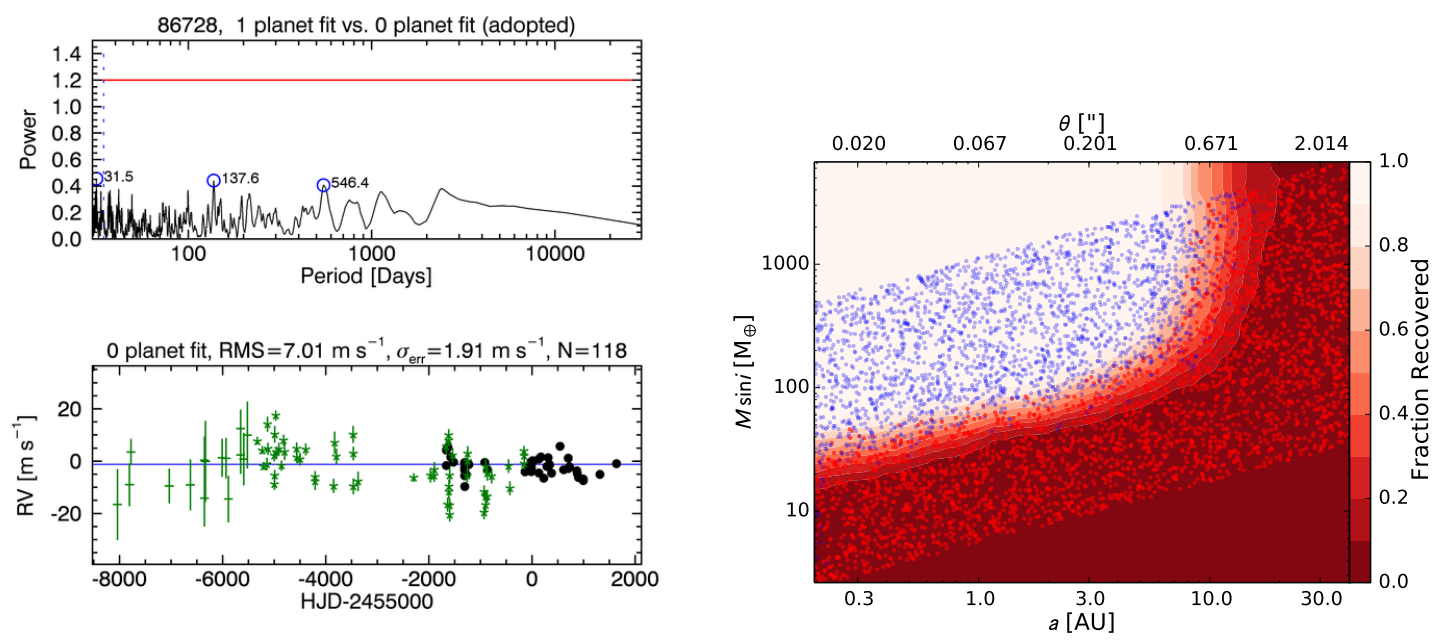

Fig. C.34.- Results from an automated search for planets orbiting the star HD 86728 (HIP 49081; programs = S, A) based on RVs from Lick and/or Keck Observatory. The set of plots on the left (analogous to Figures 4 and 5) show the planet search results and the plot on the right shows the completeness limits (analogous to Fig. 6). See the captions of those figures for detailed descriptions. 

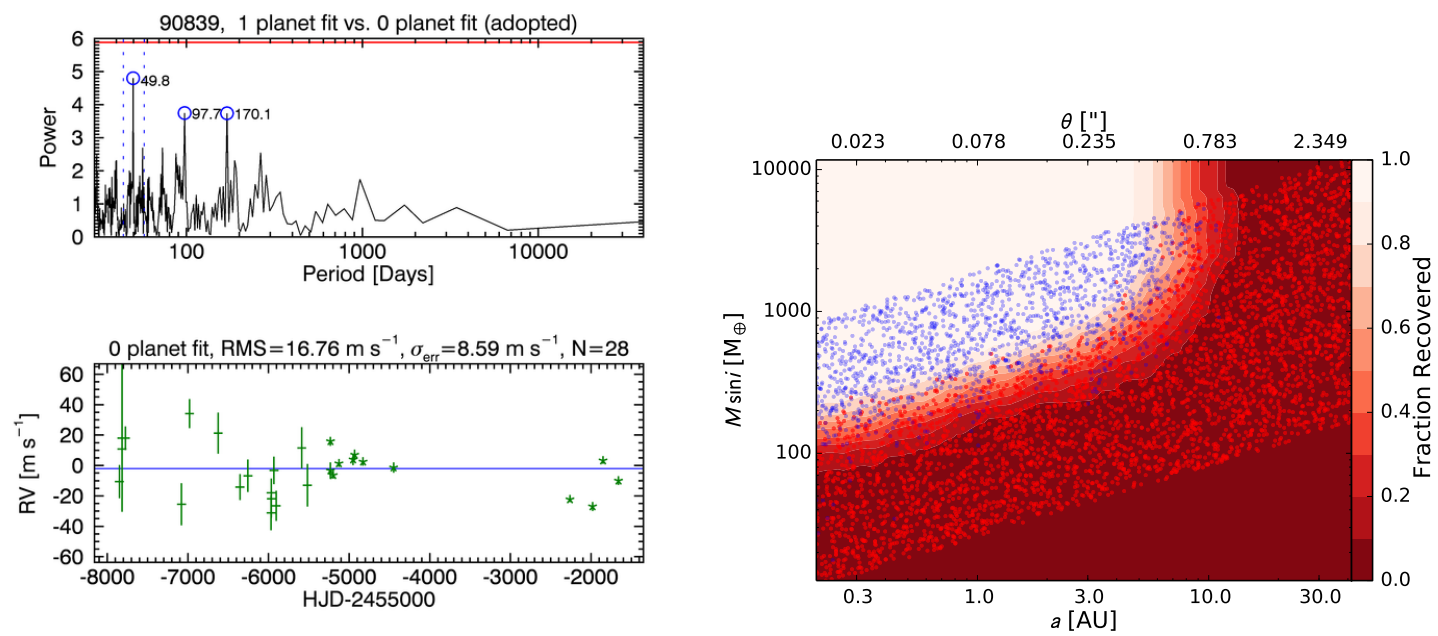

Fig. C.35. - Results from an automated search for planets orbiting the star HD 90839 (HIP 51459; programs = S, C, A) based on RVs from Lick and/or Keck Observatory. The set of plots on the left (analogous to Figures 4 and 5 ) show the planet search results and the plot on the right shows the completeness limits (analogous to Fig. 6). See the captions of those figures for detailed descriptions. 

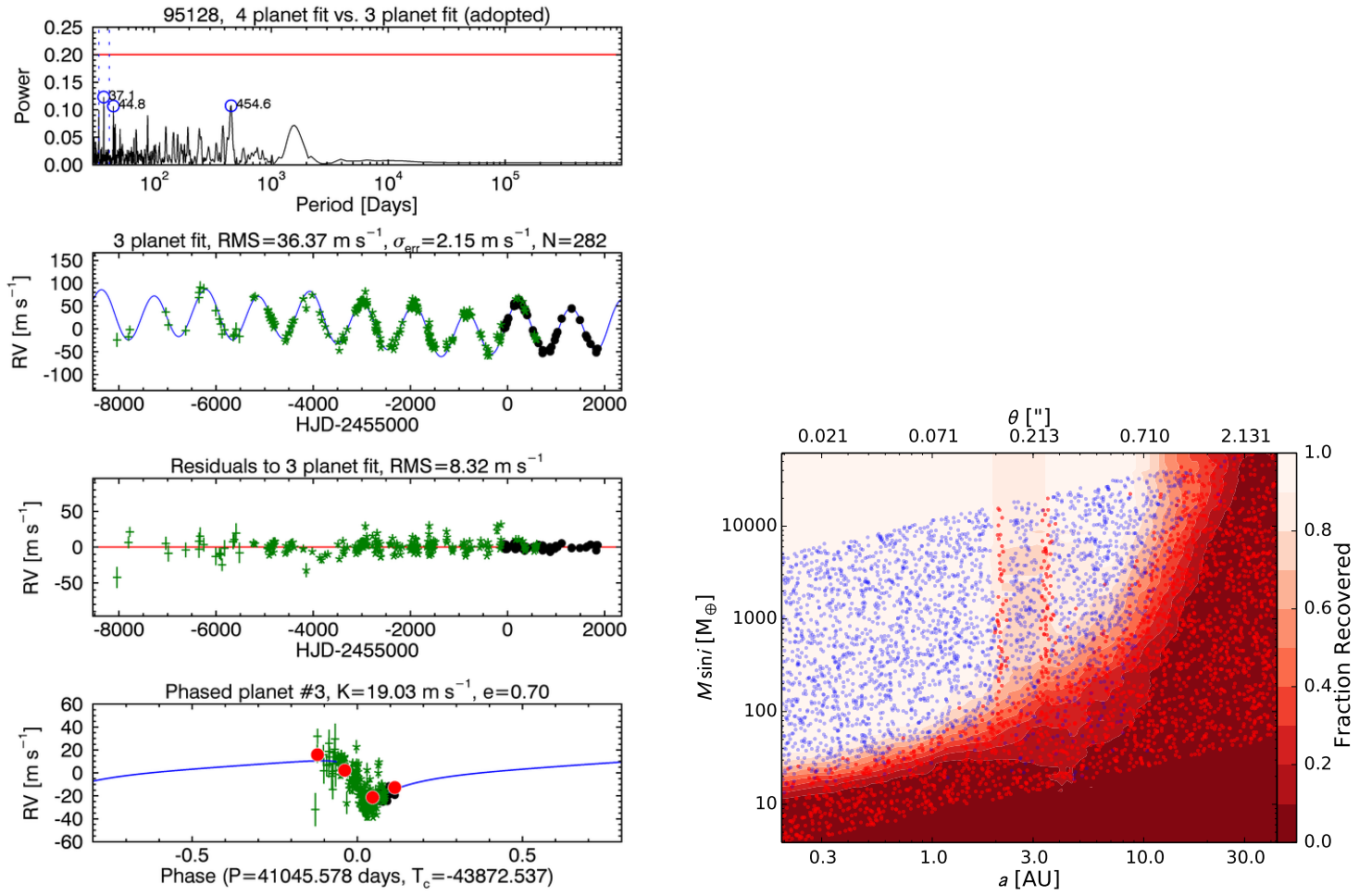

Fig. C.36. - Results from an automated search for planets orbiting the star HD 95128 (HIP 53721; programs = A) based on RVs from Lick and/or Keck Observatory. The set of plots on the left (analogous to Figures 4 and 5) show the planet search results and the plot on the right shows the completeness limits (analogous to Fig. 6). See the captions of those figures for detailed descriptions. This star hosts two well-known giant planets with semimajor axes of 2 and $3.6 \mathrm{AU}$, and possibly a third planet at 11 AU. Our automated search prefers a model with three planets, although the outer most planet has a poorly constrained orbit. 

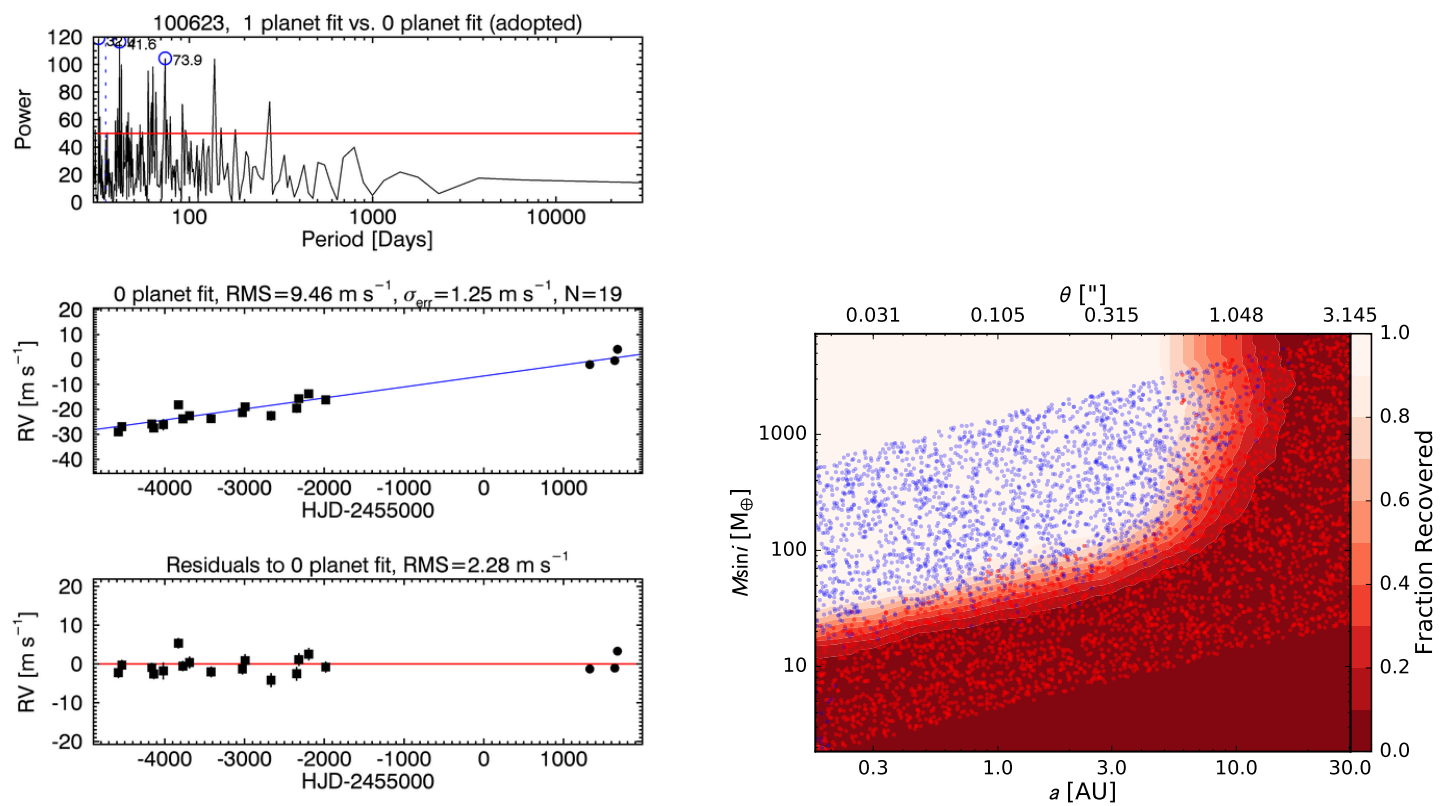

Fig. C.37.- Results from an automated search for planets orbiting the star HD 100623 (HIP 56452; programs = S) based on RVs from Lick and/or Keck Observatory. The set of plots on the left (analogous to Figures 4 and 5 ) show the planet search results and the plot on the right shows the completeness limits (analogous to Fig. 6). See the captions of those figures for detailed descriptions. This star shows a significant linear trend with no detectable curvature. 

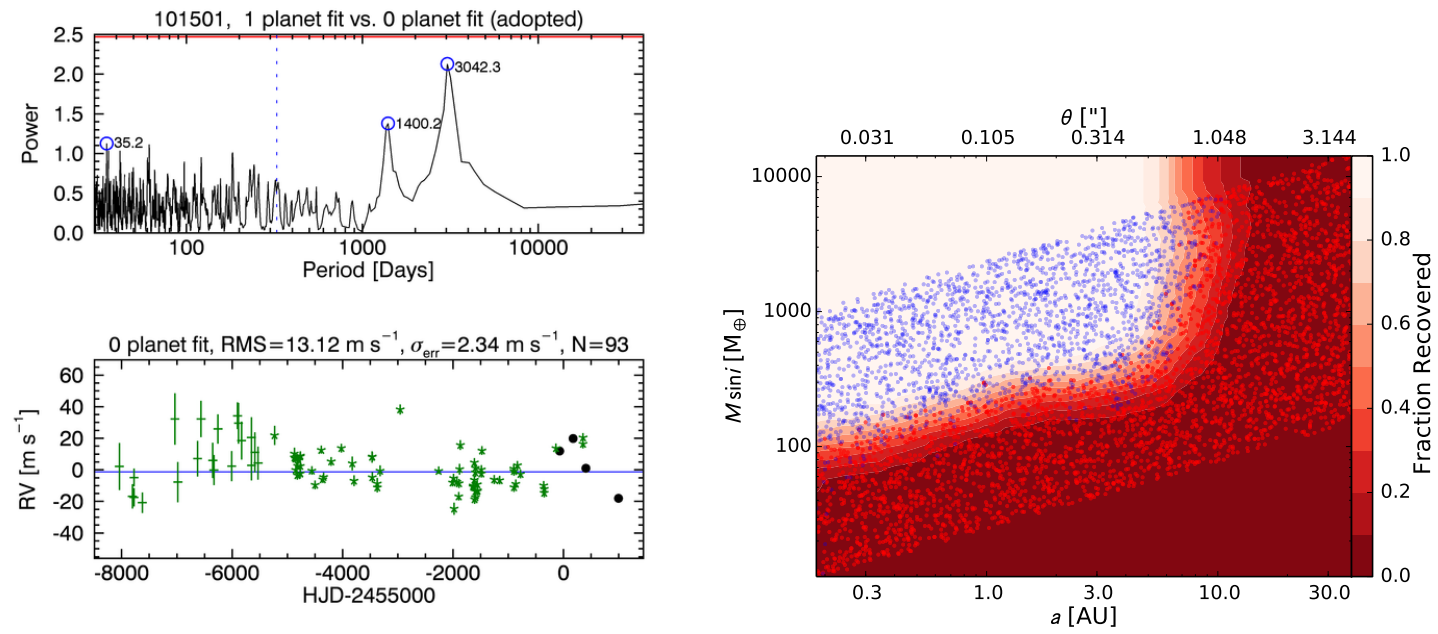

Fig. C.38. - Results from an automated search for planets orbiting the star HD 101501 (HIP 56997; programs = S, C, A) based on RVs from Lick and/or Keck Observatory. The set of plots on the left (analogous to Figures 4 and 5 show the planet search results and the plot on the right shows the completeness limits (analogous to Fig. 6). See the captions of those figures for detailed descriptions. 

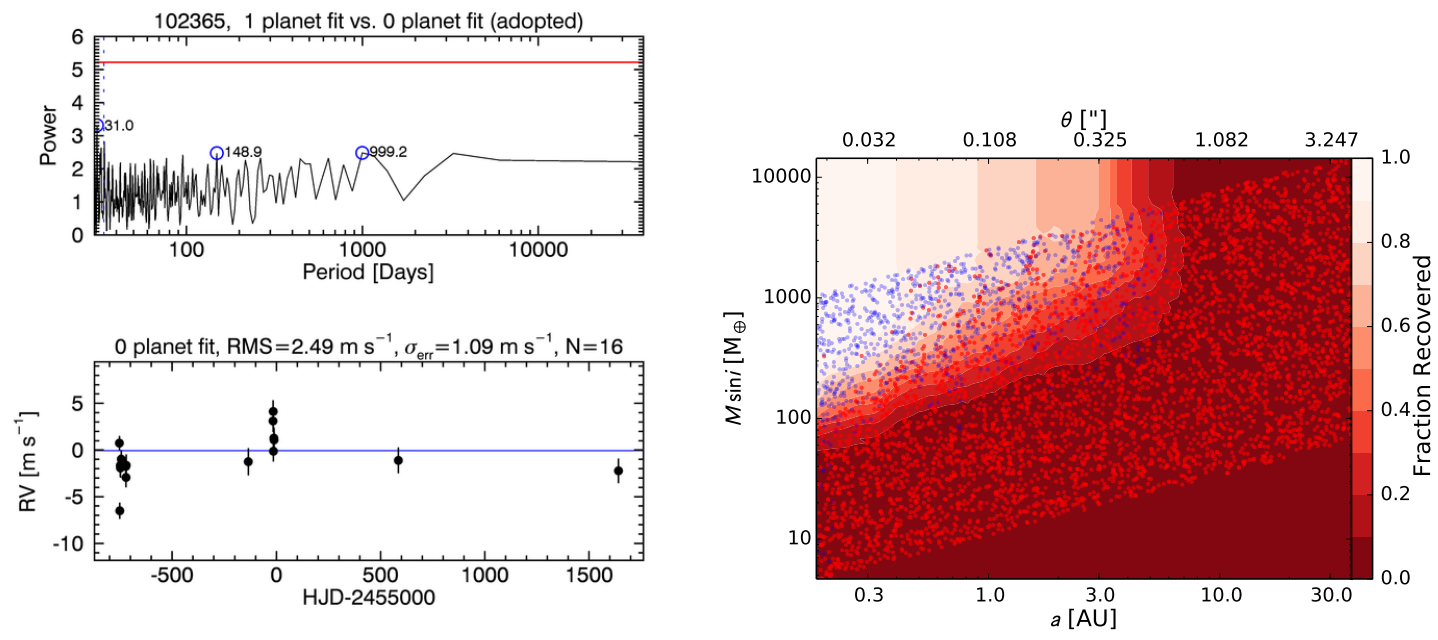

Fig. C.39.- Results from an automated search for planets orbiting the star HD 102365 (HIP 57443; programs = S, C, A) based on RVs from Lick and/or Keck Observatory. The set of plots on the left (analogous to Figures 4 and 5) show the planet search results and the plot on the right shows the completeness limits (analogous to Fig. 6). See the captions of those figures for detailed descriptions.
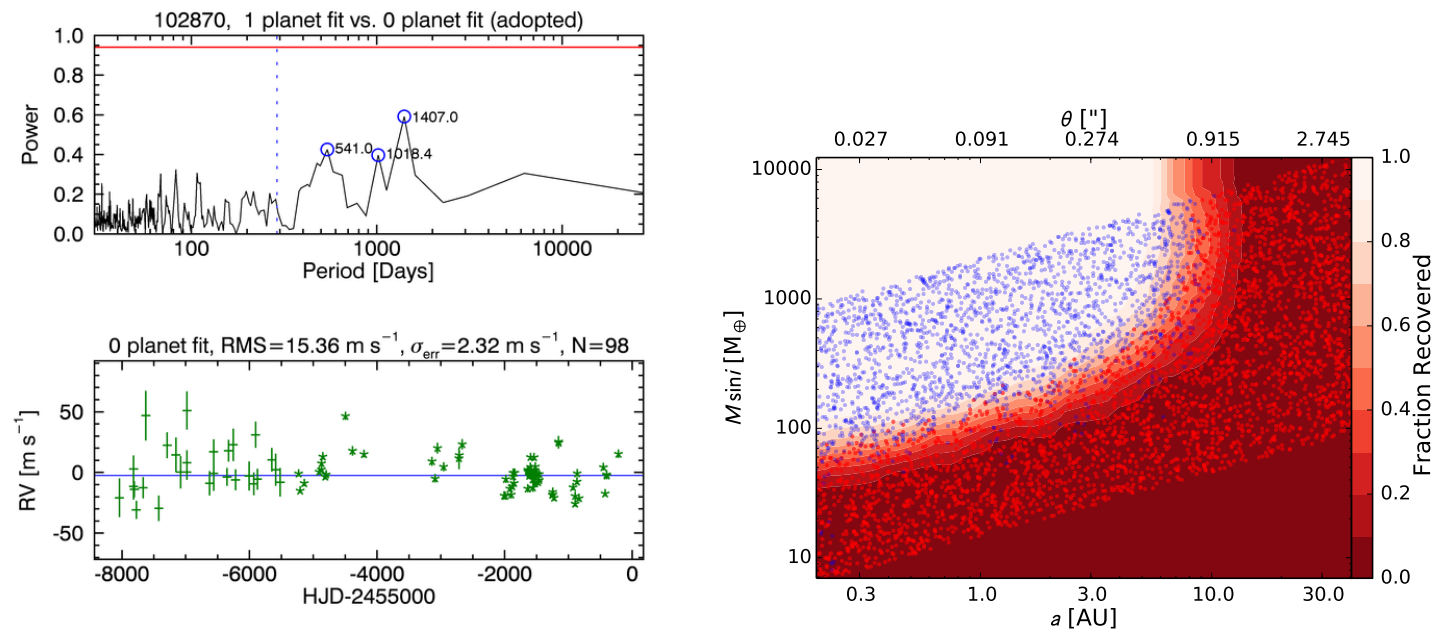

Fig. C.40.- Results from an automated search for planets orbiting the star HD 102870 (HIP 57757; programs = S, C, A) based on RVs from Lick and/or Keck Observatory. The set of plots on the left (analogous to Figures 4 and 5 ) show the planet search results and the plot on the right shows the completeness limits (analogous to Fig. 6). See the captions of those figures for detailed descriptions. 

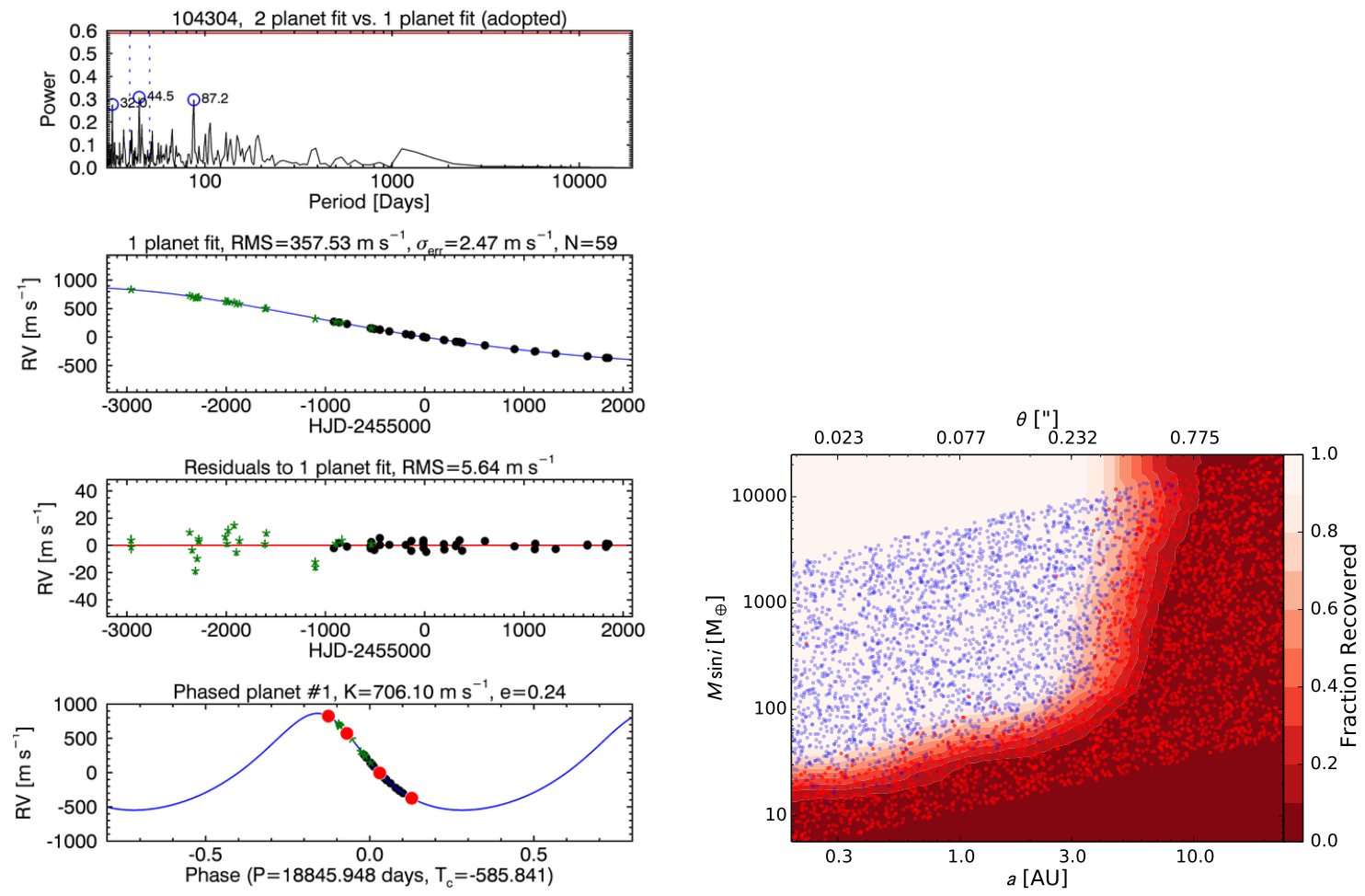

Fig. C.41. - Results from an automated search for planets orbiting the star HD 104304 (HIP 58576; program $=\mathrm{S}$ ) based on RVs from Lick and/or Keck Observatory. The set of plots on the left (analogous to Figures 4 and 5) show the planet search results and the plot on the right shows the completeness limits (analogous to Fig. 6). See the captions of those figures for detailed descriptions. This system shows a strong long-term linear trend with curvature, likely due to a detected low-mass, stellar companion. Our automated search prefers a model with a linear velocity trend (constant acceleration) in addition to the orbit segment from the companion (three bodies total), with considerable model degeneracy. 

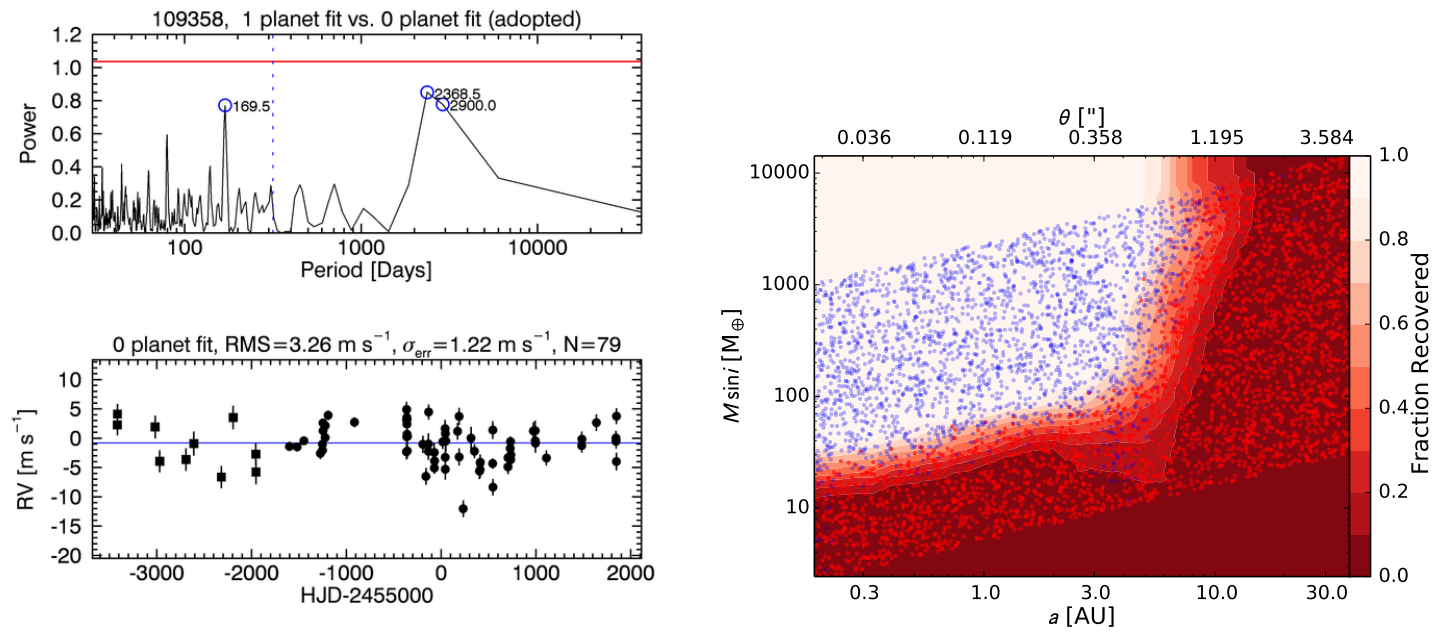

Fig. C.42.- Results from an automated search for planets orbiting the star HD 109358 (HIP 61317; programs = S, C, A) based on RVs from Lick and/or Keck Observatory. The set of plots on the left (analogous to Figures 4 and 5) show the planet search results and the plot on the right shows the completeness limits (analogous to Fig. 6). See the captions of those figures for detailed descriptions. 

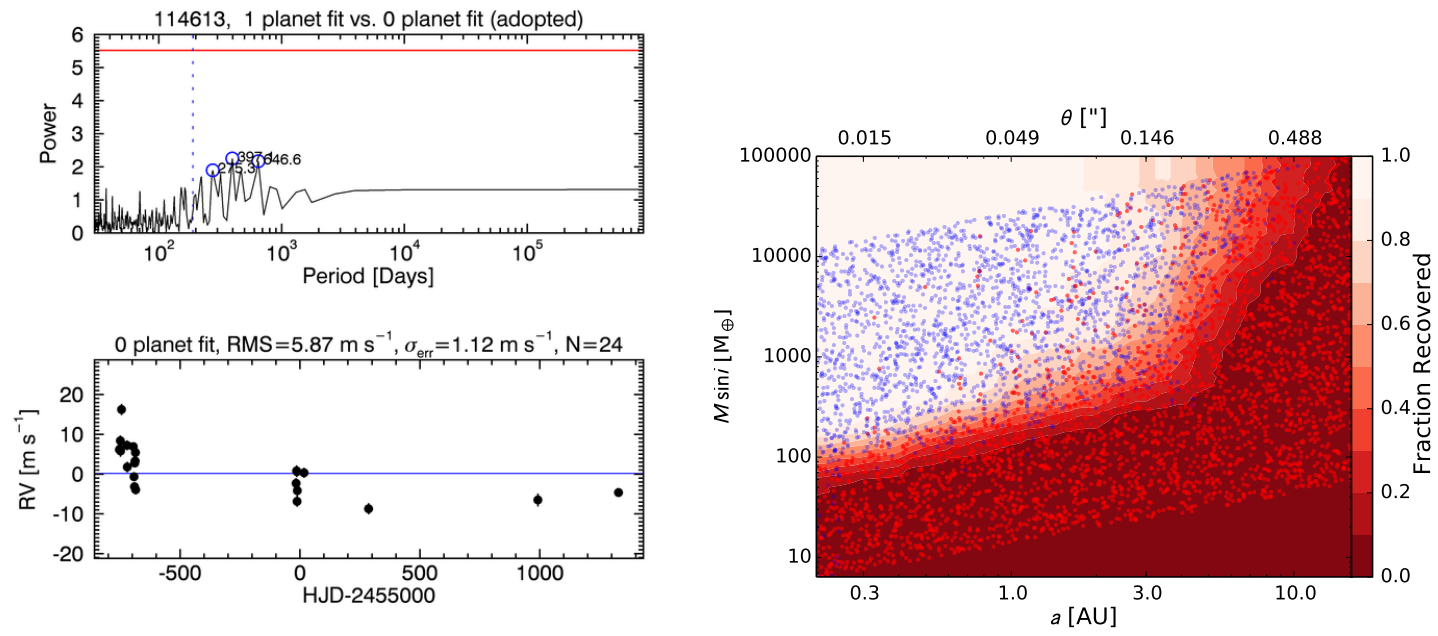

Fig. C.43.- Results from an automated search for planets orbiting the star HD 114613 (HIP 64408; program = A) based on RVs from Lick and/or Keck Observatory. The set of plots on the left (analogous to Figures 4 and 5) show the planet search results and the plot on the right shows the completeness limits (analogous to Fig. 6). See the captions of those figures for detailed descriptions.
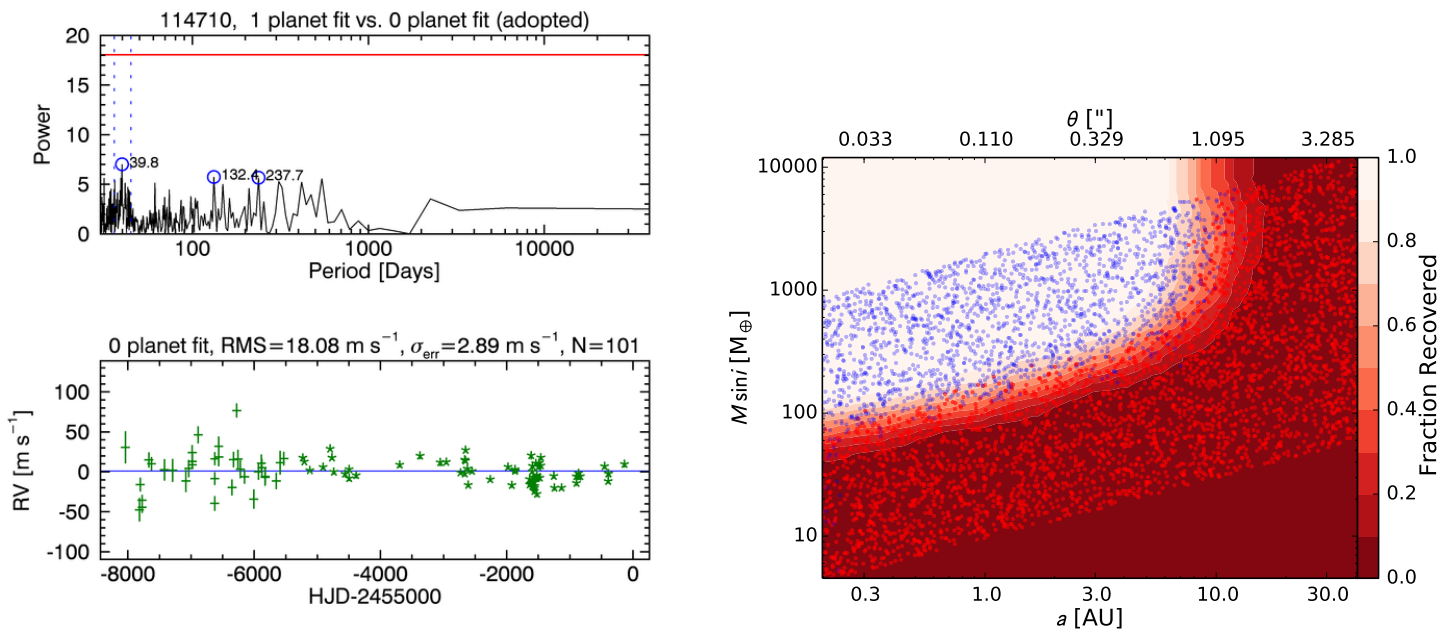

Fig. C.44. - Results from an automated search for planets orbiting the star HD 114710 (HIP 64394; programs = S, C, A) based on RVs from Lick and/or Keck Observatory. The set of plots on the left (analogous to Figures 4 and 5 show the planet search results and the plot on the right shows the completeness limits (analogous to Fig. 6). See the captions of those figures for detailed descriptions. 

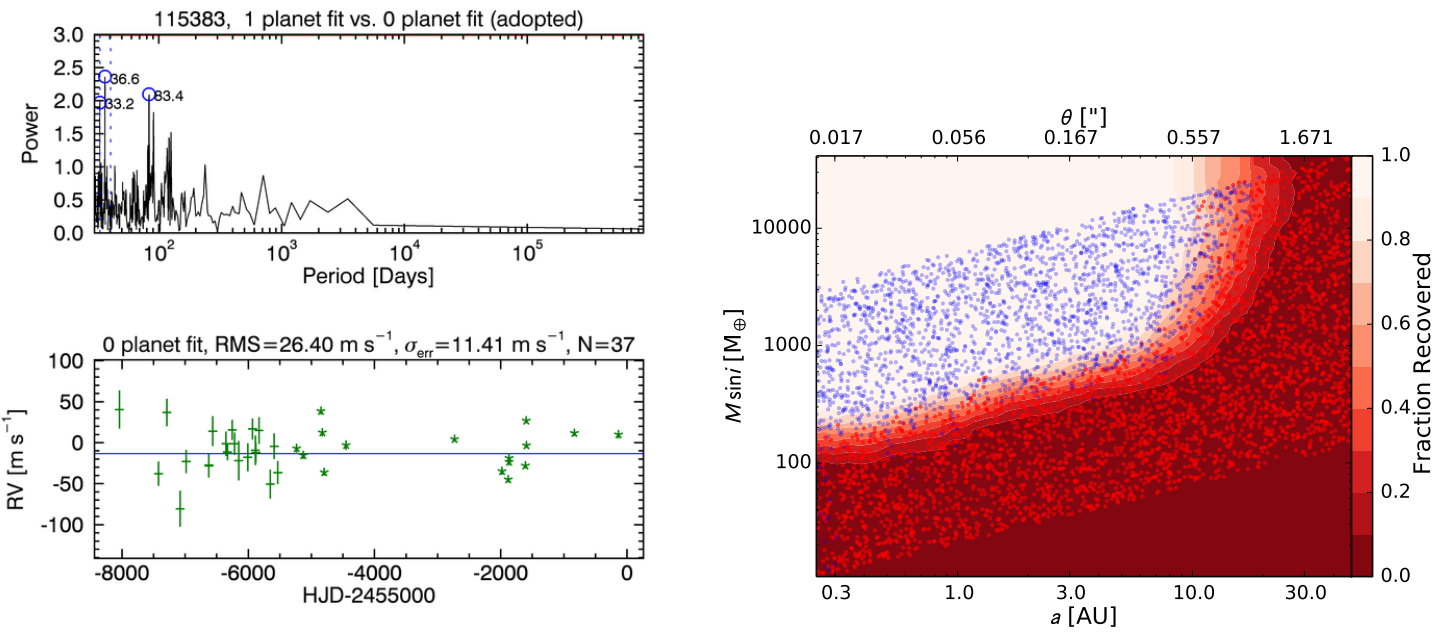

Fig. C.45. - Results from an automated search for planets orbiting the star HD 115383 (HIP 64792; program = A) based on RVs from Lick and/or Keck Observatory. The set of plots on the left (analogous to Figures 4 and 5) show the planet search results and the plot on the right shows the completeness limits (analogous to Fig. 6). See the captions of those figures for detailed descriptions. 

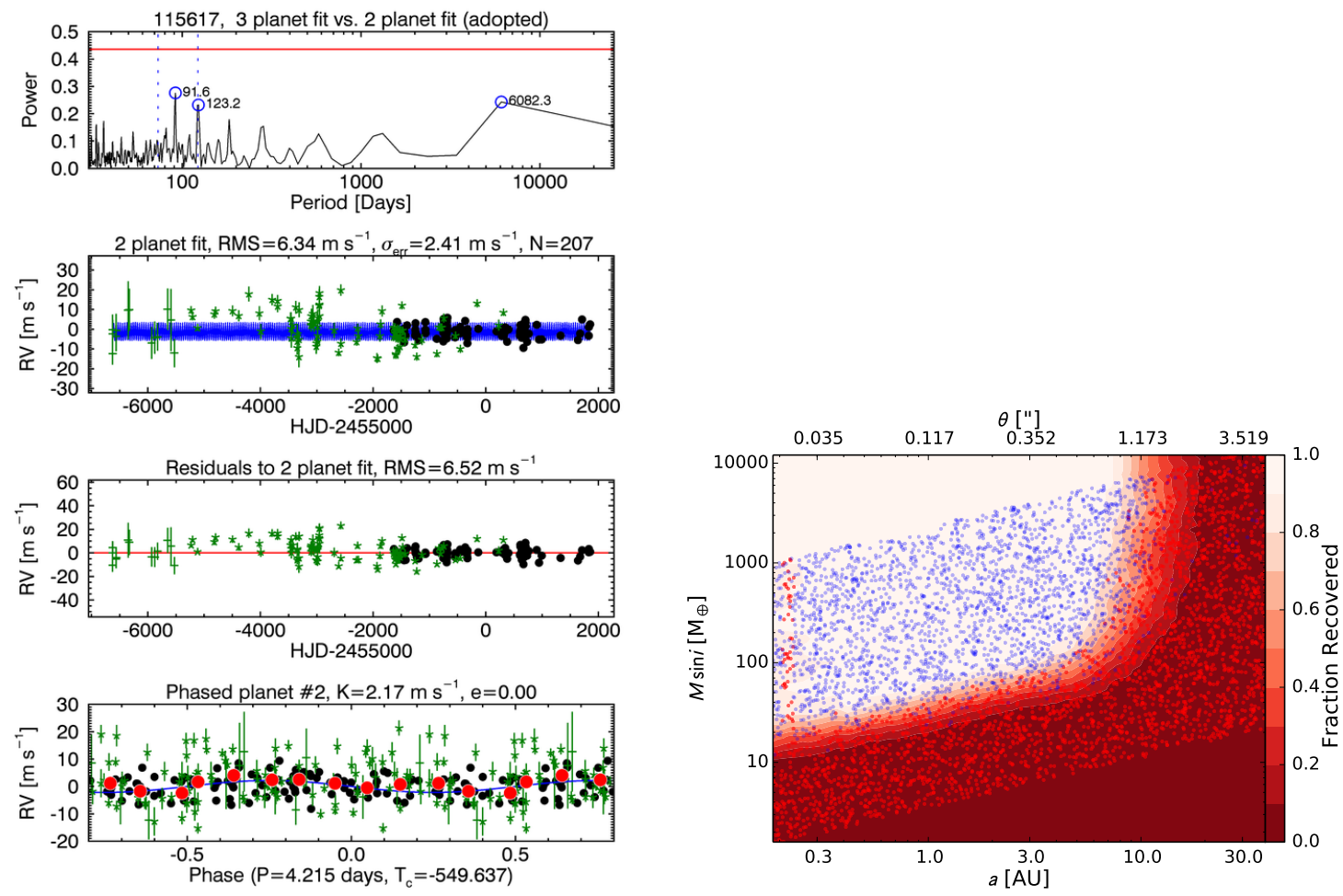

Fig. C.46. - Results from an automated search for planets orbiting the star HD 115617 (HIP 64924; program = S, C, A) based on RVs from Lick and/or Keck Observatory. The set of plots on the left (analogous to Figures 4 and 5 show the planet search results and the plot on the right shows the completeness limits (analogous to Fig. 6). See the captions of those figures for detailed descriptions. This star has three reported small planets, two of which we detect in our Keck RVs. 

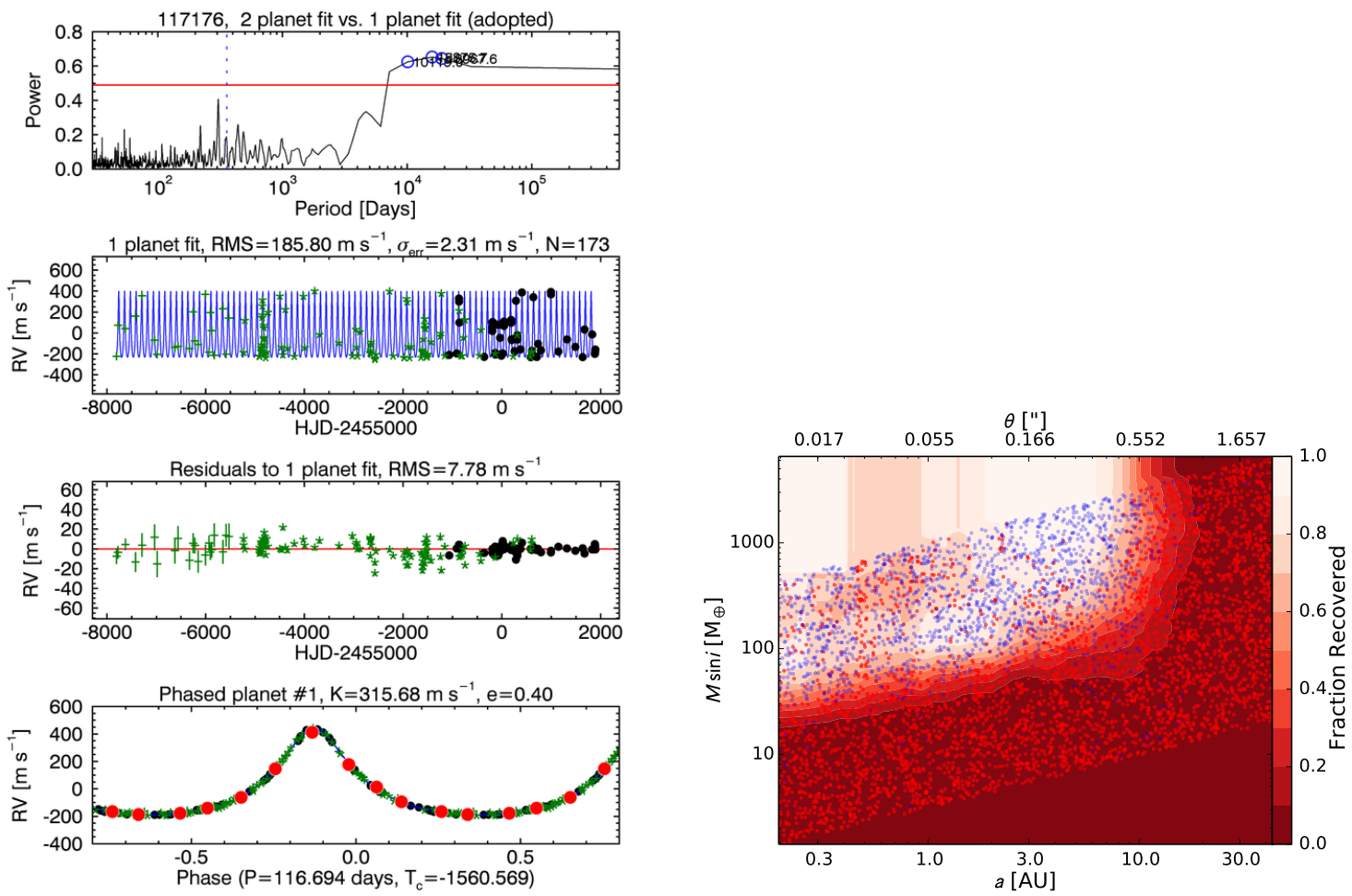

Fig. C.47.- Results from an automated search for planets orbiting the star HD 117176 $($ HIP 65721; program = A) based on RVs from Lick and/or Keck Observatory. The set of plots on the left (analogous to Figures 4 and 5) show the planet search results and the plot on the right shows the completeness limits (analogous to Fig. 6). See the captions of those figures for detailed descriptions. This star hosts a giant planet. 

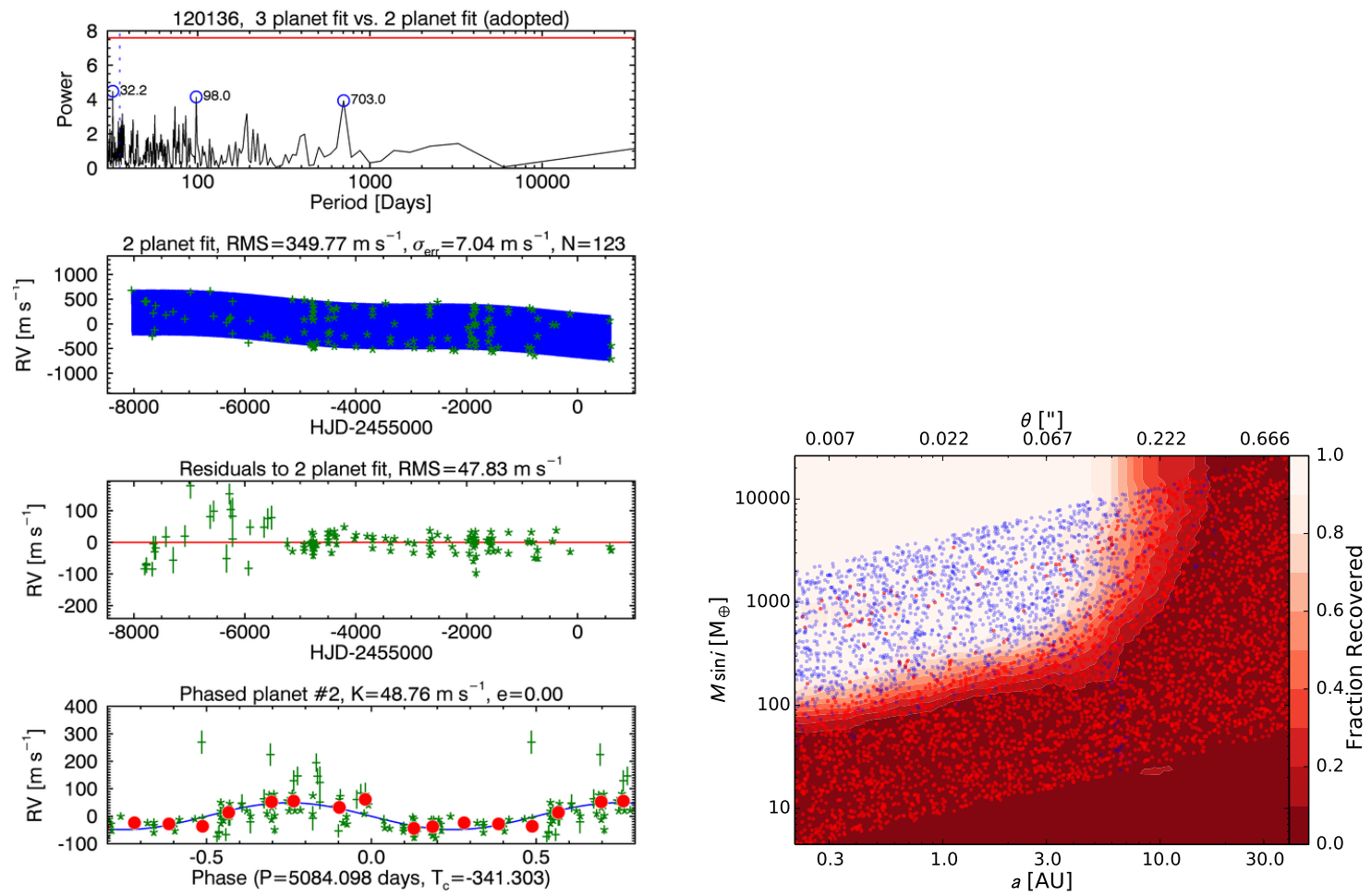

Fig. C.48. - Results from an automated search for planets orbiting the star HD 120136 (HIP 67275; programs = S, C, A) based on RVs from Lick and/or Keck Observatory. The set of plots on the left (analogous to Figures 4 and 5 show the planet search results and the plot on the right shows the completeness limits (analogous to Fig. 6). See the captions of those figures for detailed descriptions. This star hosts a massive, hot Jupiter planet. We also detect a significant linear trend in the RV time series and a periodicity at $\sim 5000$ days. The trend is likely real, but the 5000 day periodicity could be due to instrumental offsets in the Lick-only data or a stellar magnetic activity cycle. 

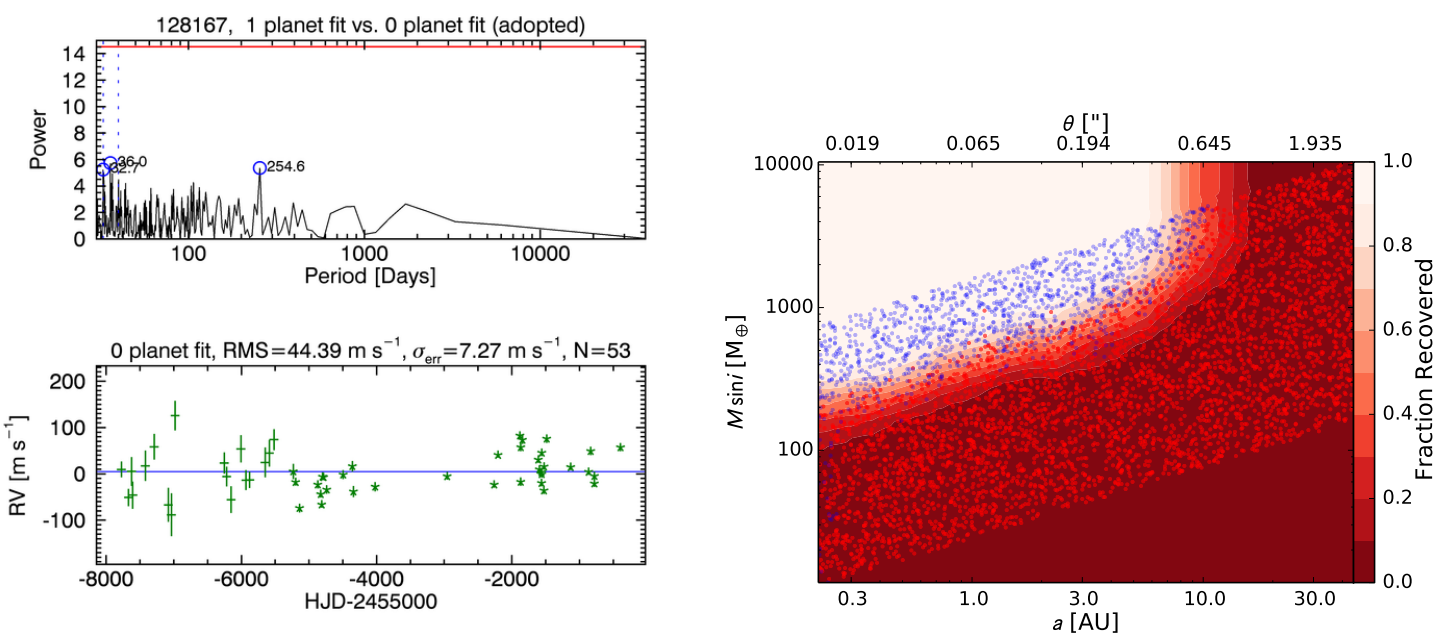

Fig. C.49.- Results from an automated search for planets orbiting the star HD 128167 (HIP 71284; programs = S, C, A) based on RVs from Lick and/or Keck Observatory. The set of plots on the left (analogous to Figures 4 and 5 show the planet search results and the plot on the right shows the completeness limits (analogous to Fig. 6). See the captions of those figures for detailed descriptions. 

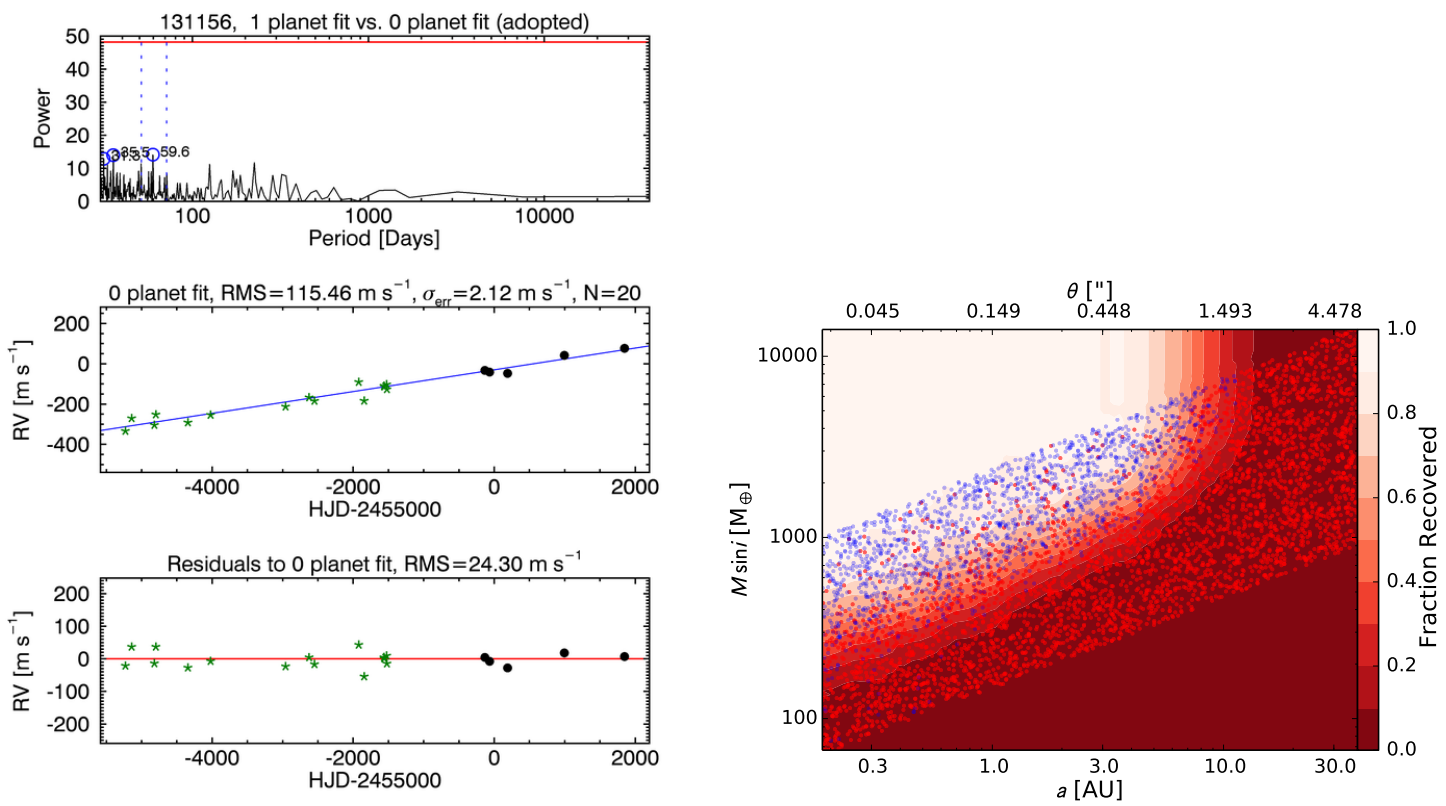

Fig. C.50.- Results from an automated search for planets orbiting the star HD 131156 (HIP 72659; programs = S, C, A) based on RVs from Lick and/or Keck Observatory. The set of plots on the left (analogous to Figures 4 and 5 ) show the planet search results and the plot on the right shows the completeness limits (analogous to Fig. 6). See the captions of those figures for detailed descriptions. This star has a significant linear trend in the RV time series, likely due to a binary companion. 

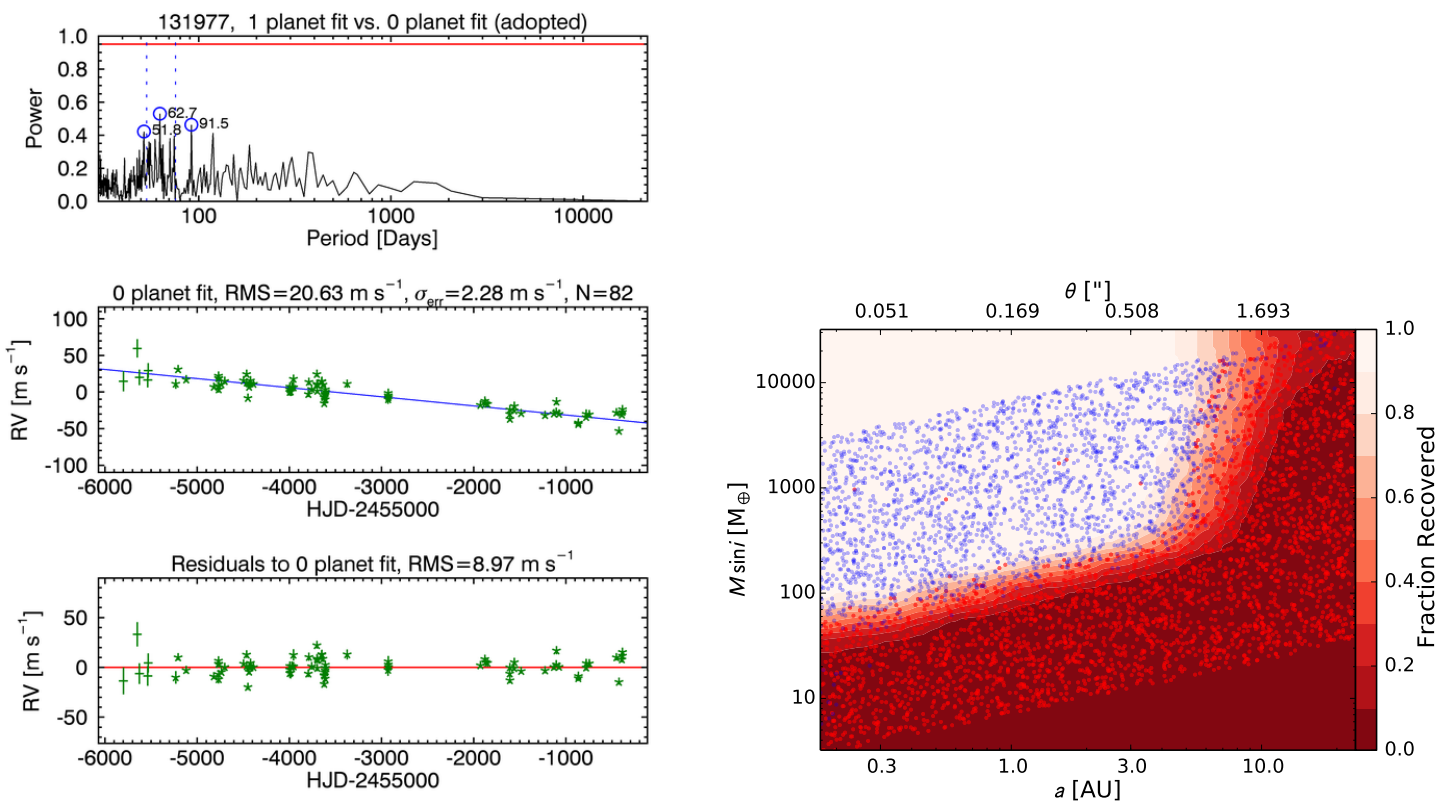

Fig. C.51. - Results from an automated search for planets orbiting the star HD 131977 (HIP 73184; programs = S, C) based on RVs from Lick and/or Keck Observatory. The set of plots on the left (analogous to Figures 4 and 5) show the planet search results and the plot on the right shows the completeness limits (analogous to Fig. 6). See the captions of those figures for detailed descriptions. This star has a significant linear trend in the RV time series, likely due to a binary companion. 

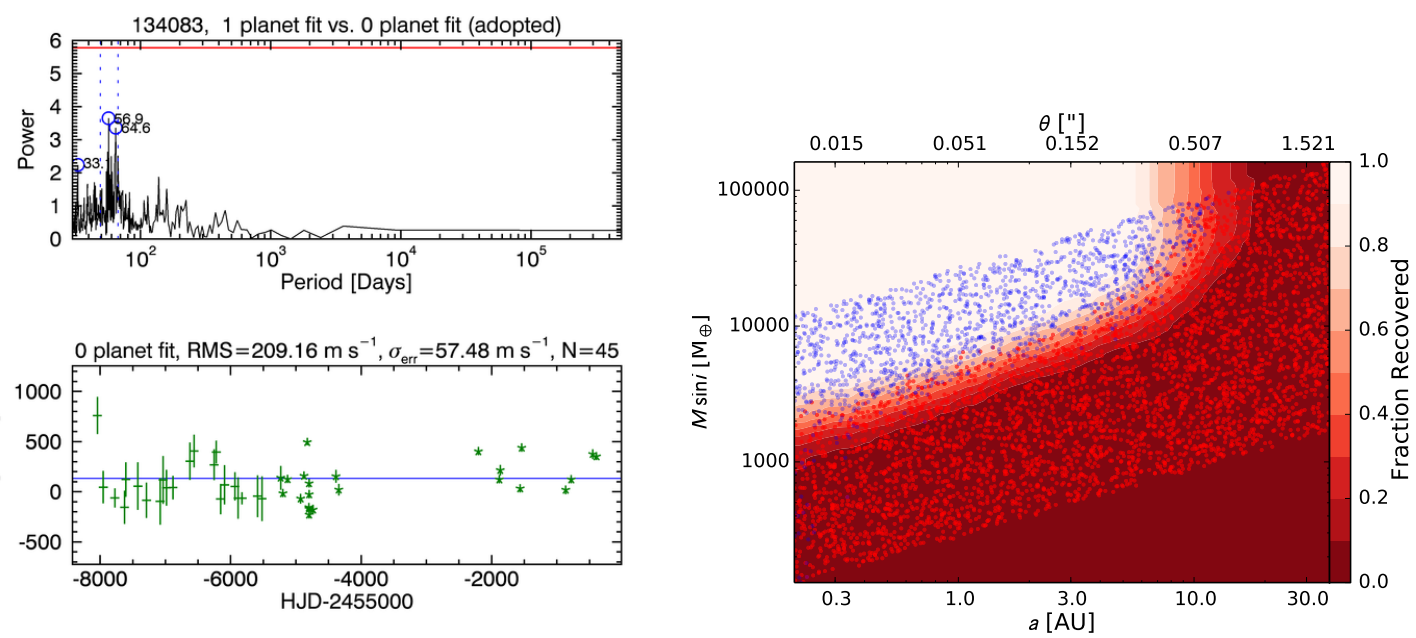

Fig. C.52.- Results from an automated search for planets orbiting the star HD 134083 (HIP 73996; program = A) based on RVs from Lick and/or Keck Observatory. The set of plots on the left (analogous to Figures 4 and 5) show the planet search results and the plot on the right shows the completeness limits (analogous to Fig. 6). See the captions of those figures for detailed descriptions. 

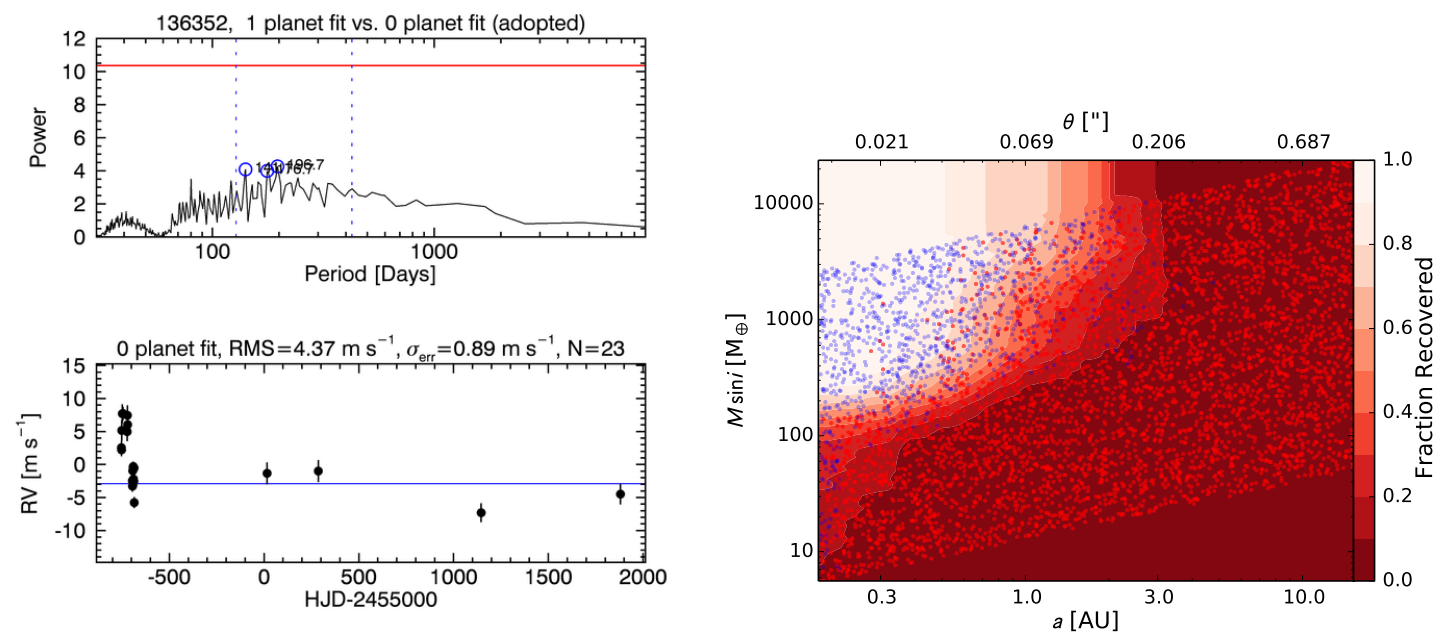

Fig. C.53. - Results from an automated search for planets orbiting the star HD 136352 $($ HIP 75181; program $=$ S) based on RVs from Lick and/or Keck Observatory. The set of plots on the left (analogous to Figures 4 and 5) show the planet search results and the plot on the right shows the completeness limits (analogous to Fig. 6). See the captions of those figures for detailed descriptions.
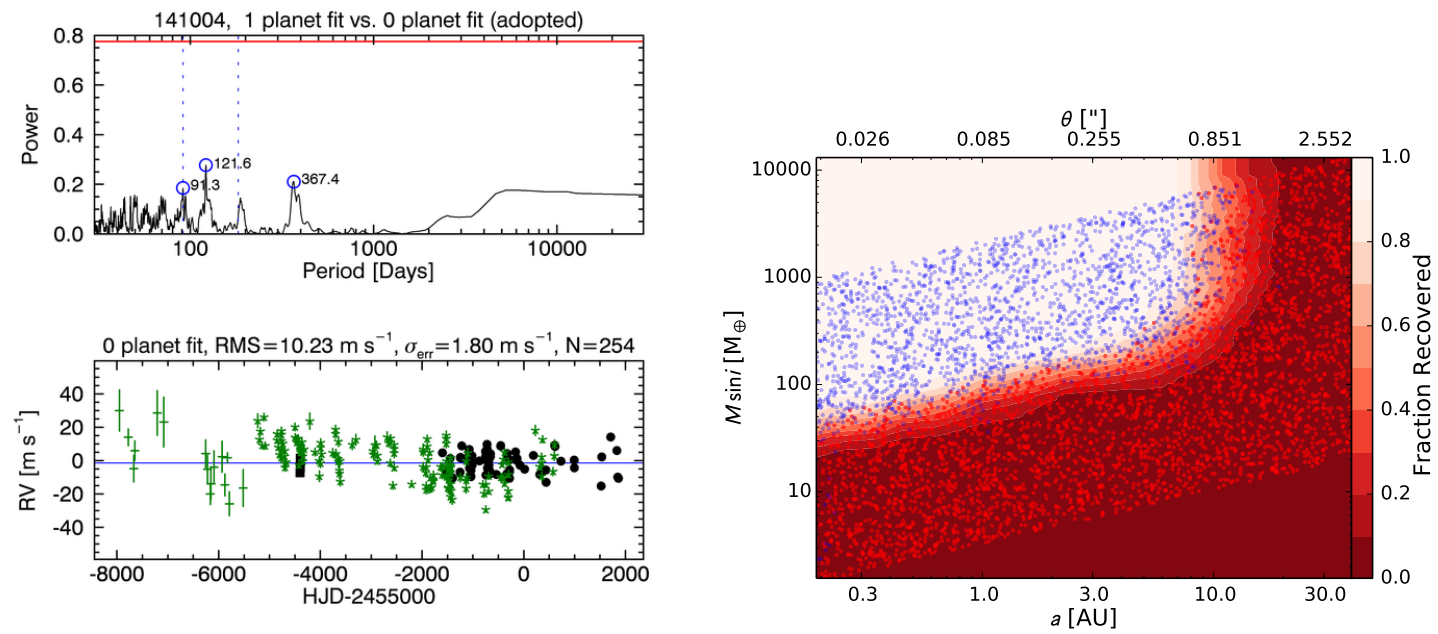

Fig. C.54. - Results from an automated search for planets orbiting the star HD 141004 (HIP 77257; programs = S, C, A) based on RVs from Lick and/or Keck Observatory. The set of plots on the left (analogous to Figures 4 and 5 show the planet search results and the plot on the right shows the completeness limits (analogous to Fig. 6). See the captions of those figures for detailed descriptions. 

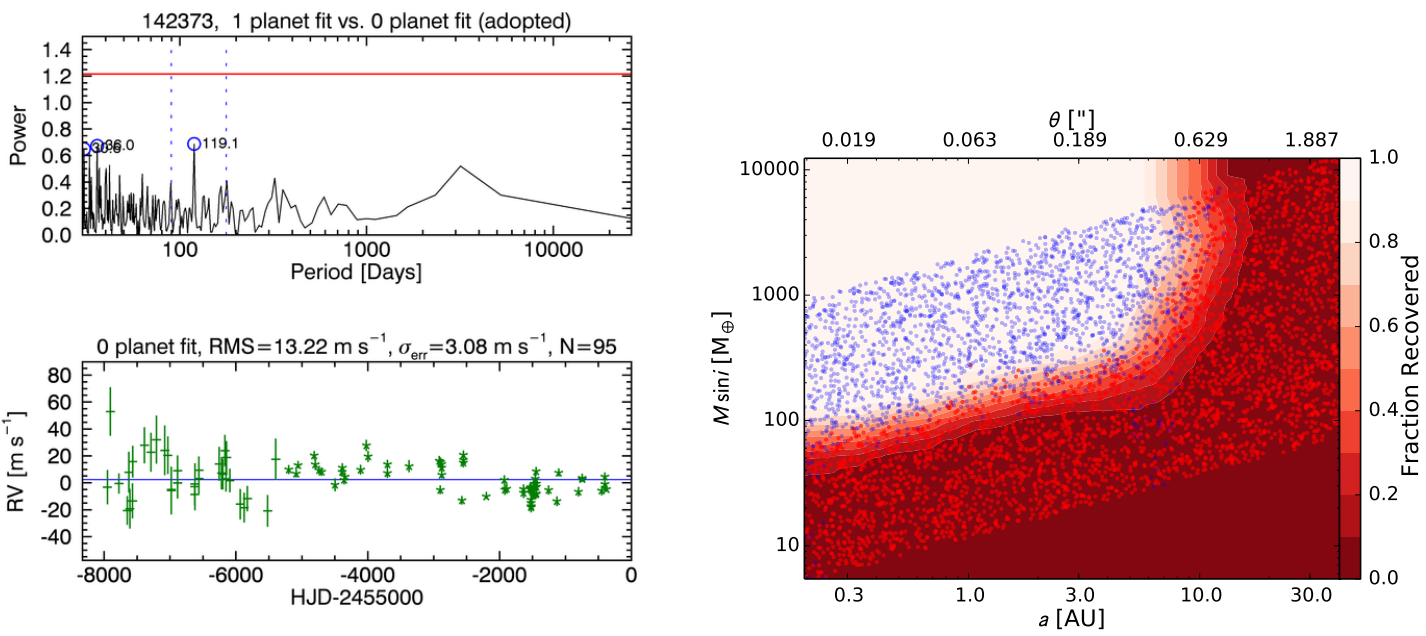

Fig. C.55. - Results from an automated search for planets orbiting the star HD 142373 (HIP 77760; programs = S, C, A) based on RVs from Lick and/or Keck Observatory. The set of plots on the left (analogous to Figures 4 and 5 show the planet search results and the plot on the right shows the completeness limits (analogous to Fig. 6). See the captions of those figures for detailed descriptions.
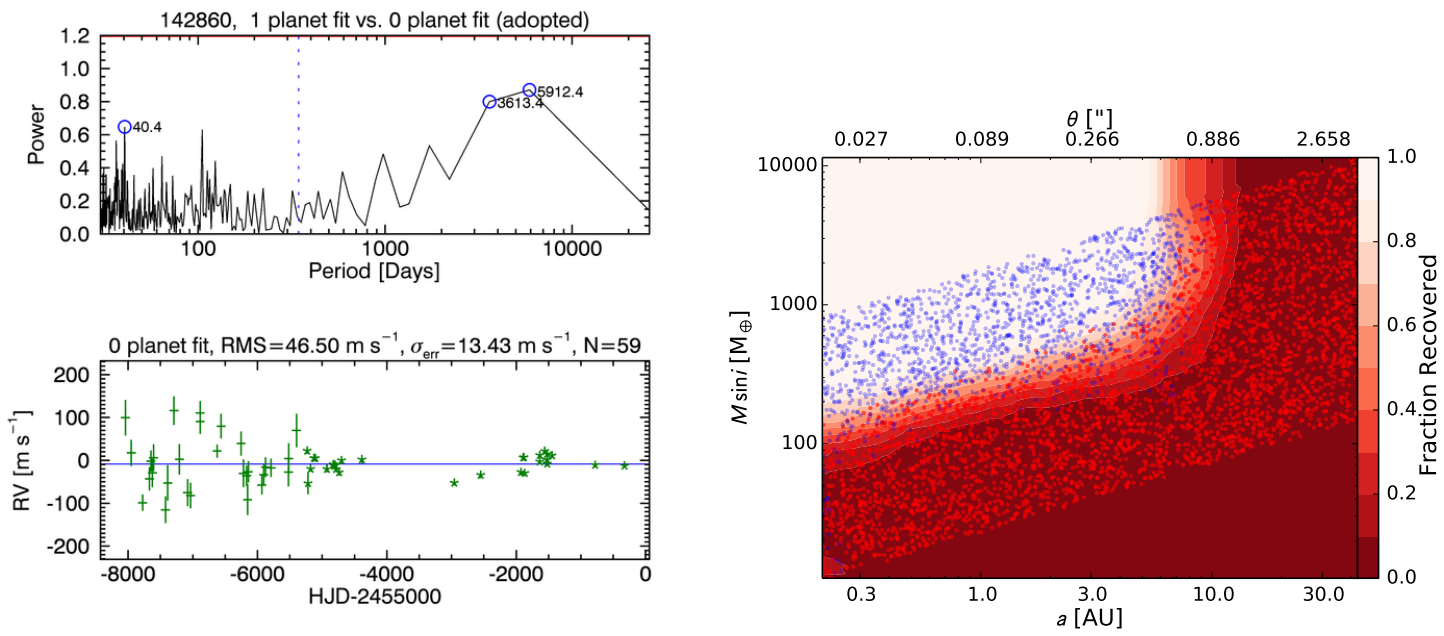

Fig. C.56. - Results from an automated search for planets orbiting the star HD 142860 (HIP 78072; programs $=$ S, C, A) based on RVs from Lick and/or Keck Observatory. The set of plots on the left (analogous to Figures 4 and 5 ) show the planet search results and the plot on the right shows the completeness limits (analogous to Fig. 6). See the captions of those figures for detailed descriptions. 

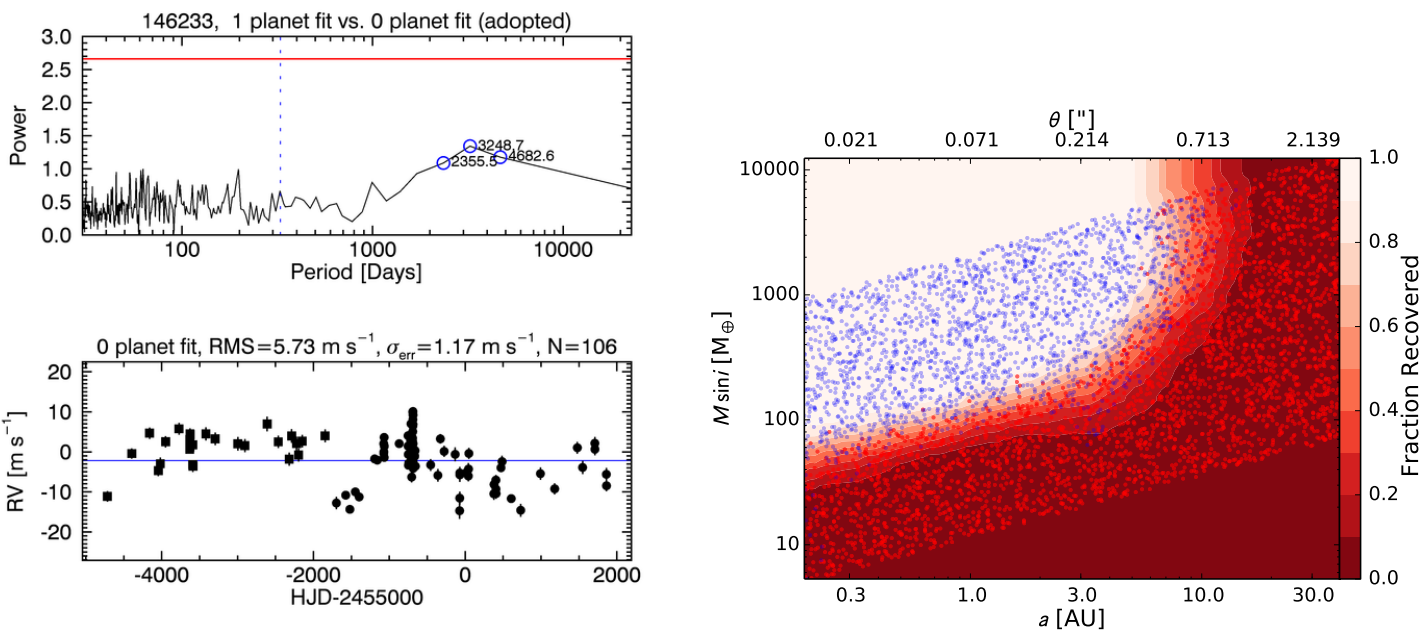

Fig. C.57.- Results from an automated search for planets orbiting the star HD 146233 $($ HIP 79672; program $=$ S) based on RVs from Lick and/or Keck Observatory. The set of plots on the left (analogous to Figures 4 and 5) show the planet search results and the plot on the right shows the completeness limits (analogous to Fig. 6). See the captions of those figures for detailed descriptions.
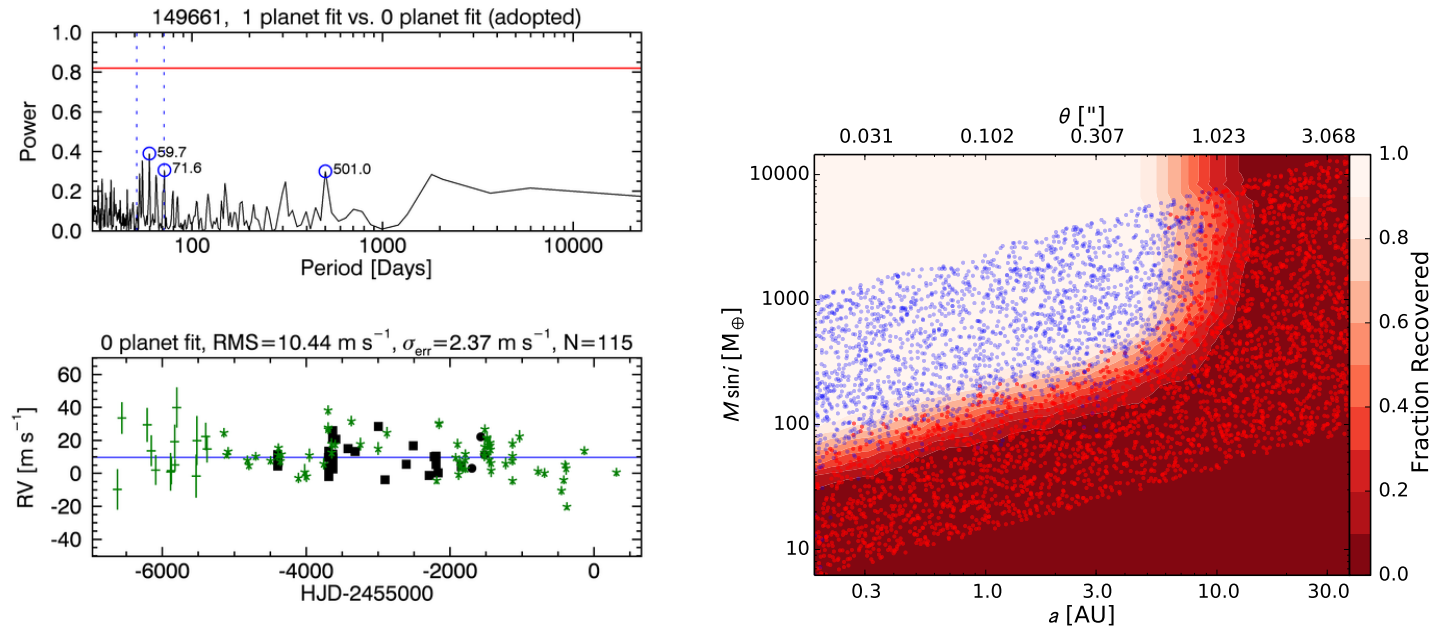

Fig. C.58. - Results from an automated search for planets orbiting the star HD 149661 $($ HIP 81300; program $=\mathrm{S}$ ) based on RVs from Lick and/or Keck Observatory. The set of plots on the left (analogous to Figures 4 and 5 show the planet search results and the plot on the right shows the completeness limits (analogous to Fig. 6). See the captions of those figures for detailed descriptions. 

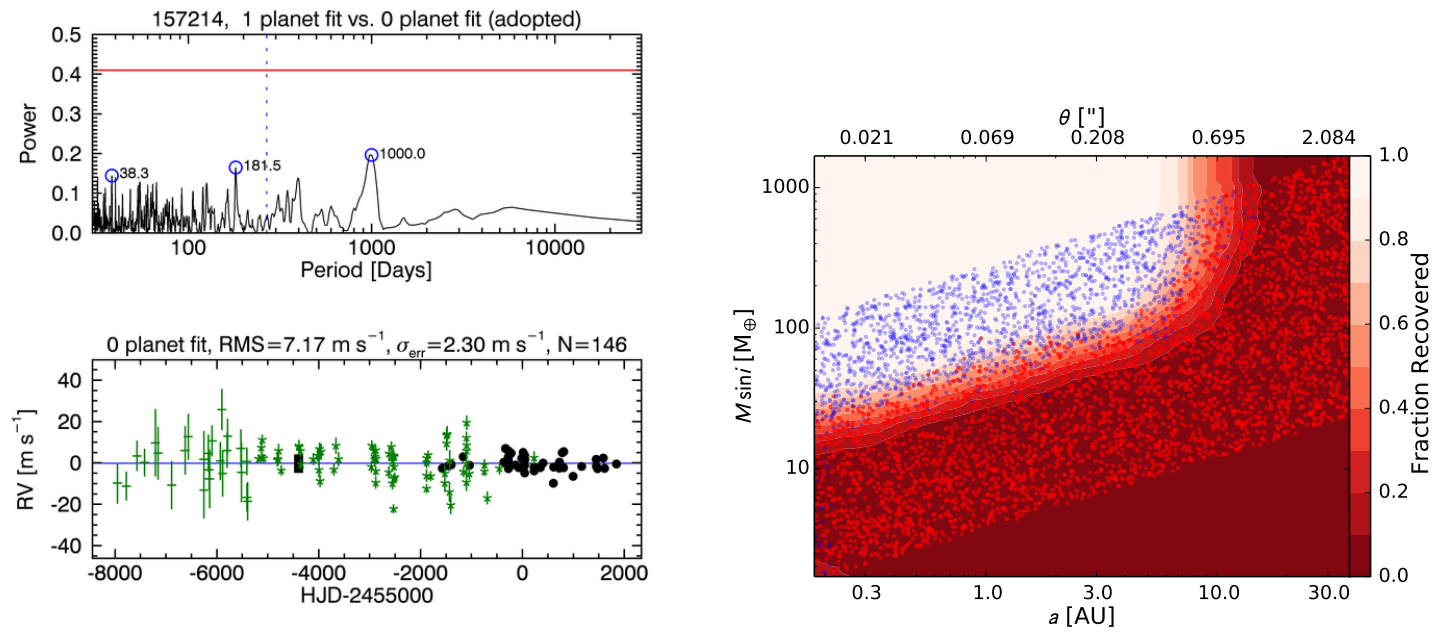

Fig. C.59. - Results from an automated search for planets orbiting the star HD 157214 (HIP 84862; programs = S, A) based on RVs from Lick and/or Keck Observatory. The set of plots on the left (analogous to Figures 4 and 5) show the planet search results and the plot on the right shows the completeness limits (analogous to Fig. 6). See the captions of those figures for detailed descriptions. 

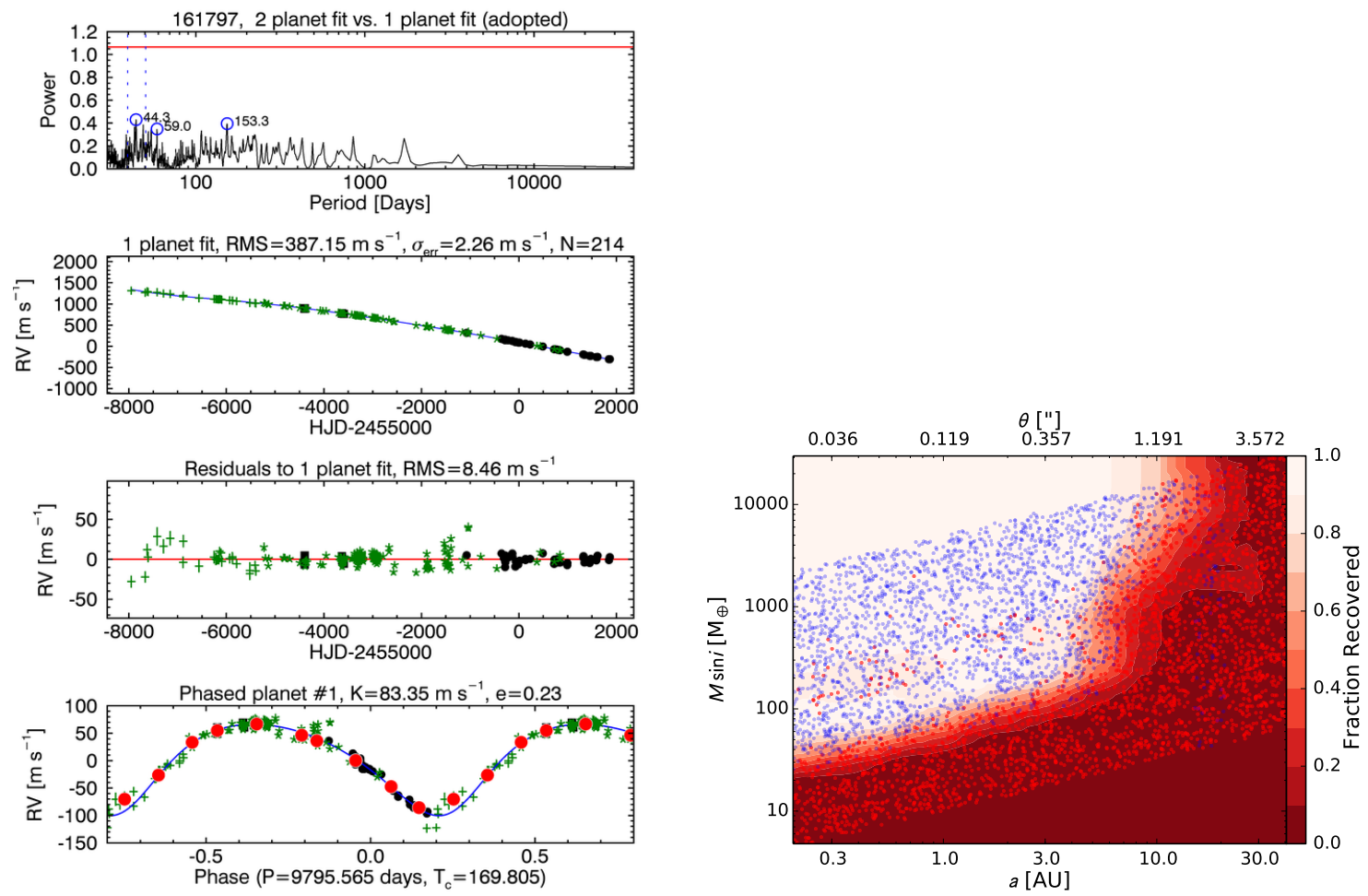

Fig. C.60.- Results from an automated search for planets orbiting the star HD 161797 (HIP 86974; programs = S, C, A) based on RVs from Lick and/or Keck Observatory. The set of plots on the left (analogous to Figures 4 and 5) show the planet search results and the plot on the right shows the completeness limits (analogous to Fig. 6). See the captions of those figures for detailed descriptions. This star is in a hierarchical triple system and shows a strong linear trend and significant curvature or a possibly closed orbit in the RV time series. 

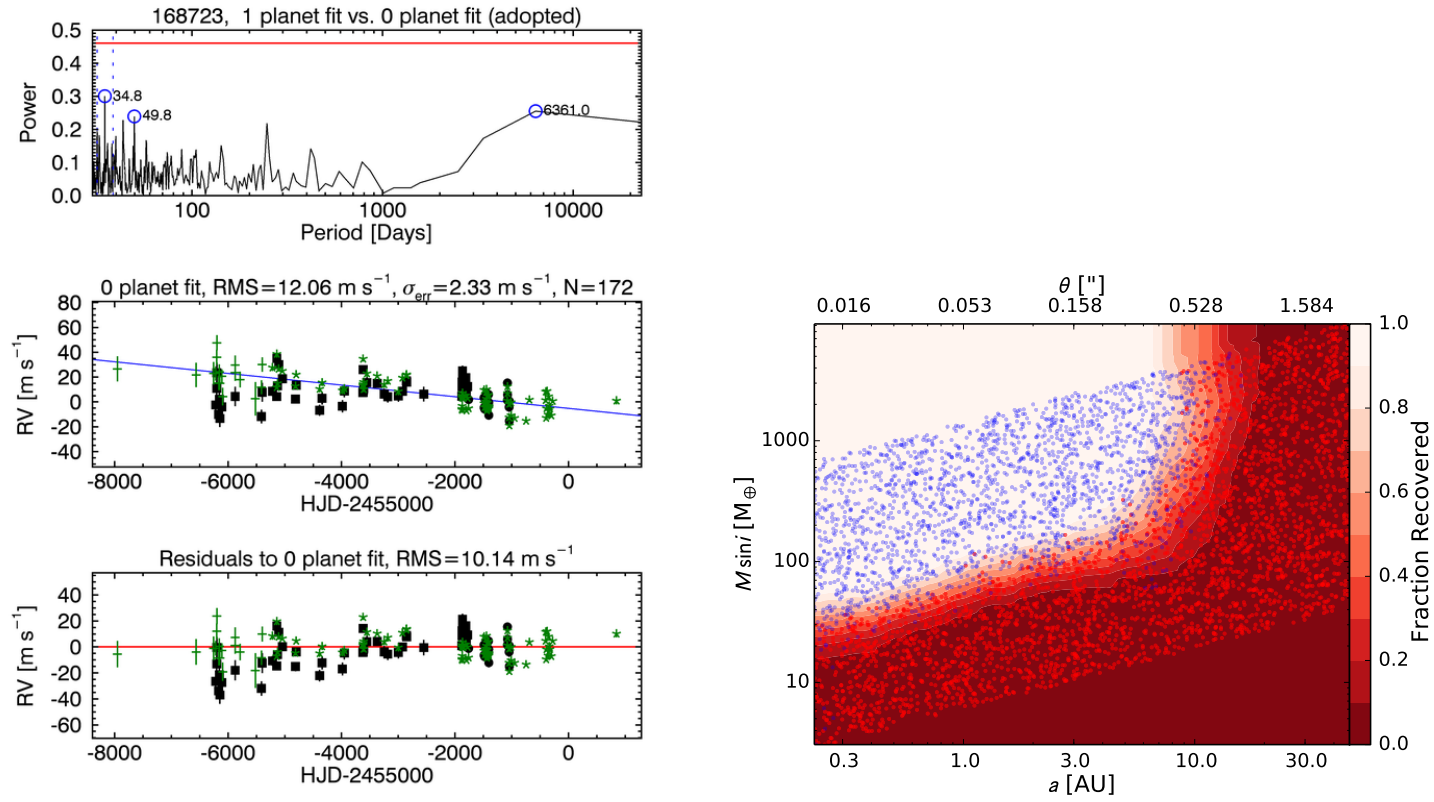

Fig. C.61. - Results from an automated search for planets orbiting the star HD 168723 (HIP 89962; program = C) based on RVs from Lick and/or Keck Observatory. The set of plots on the left (analogous to Figures 4 and 5) show the planet search results and the plot on the right shows the completeness limits (analogous to Fig. 6). See the captions of those figures for detailed descriptions. The Lick data for this star show a marginal linear trend in the RV time series while the long-baseline pre-upgrade Keck data (code $=\mathrm{k}$ ) does not show the trend. 

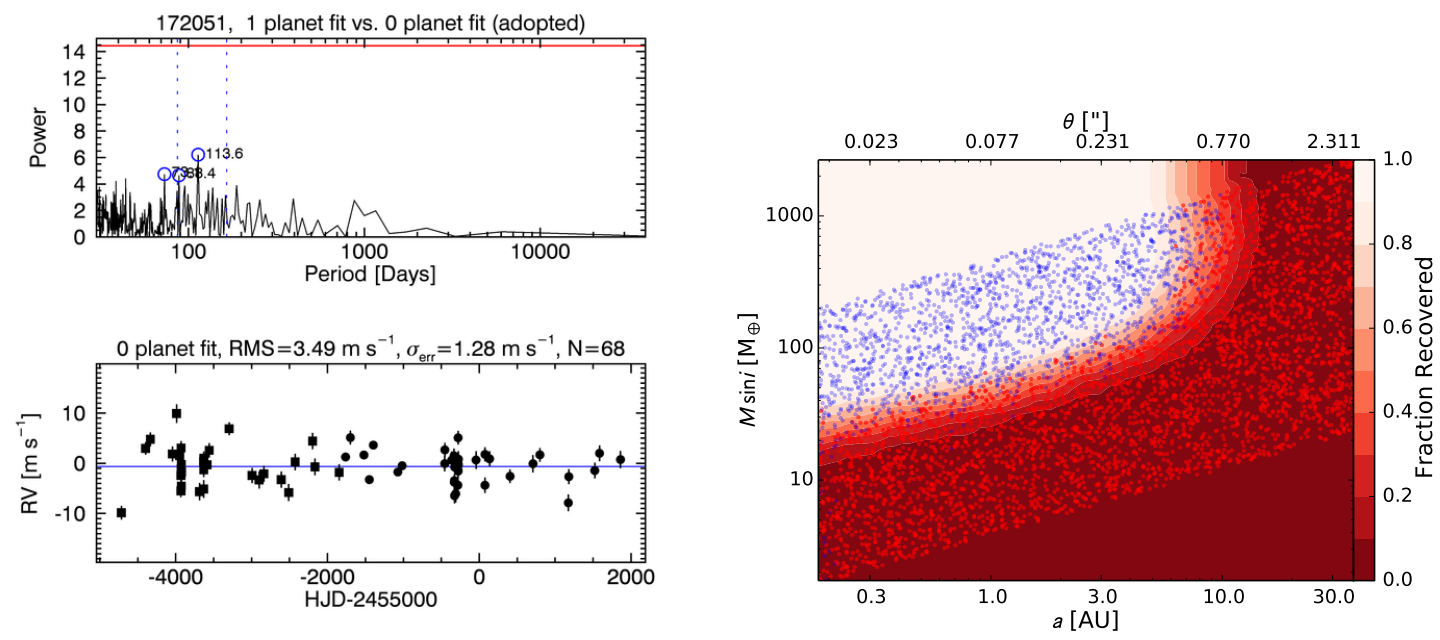

Fig. C.62.- Results from an automated search for planets orbiting the star HD 172051 (HIP 91438; program $=$ S) based on RVs from Lick and/or Keck Observatory. The set of plots on the left (analogous to Figures 4 and 5) show the planet search results and the plot on the right shows the completeness limits (analogous to Fig. 6). See the captions of those figures for detailed descriptions. 

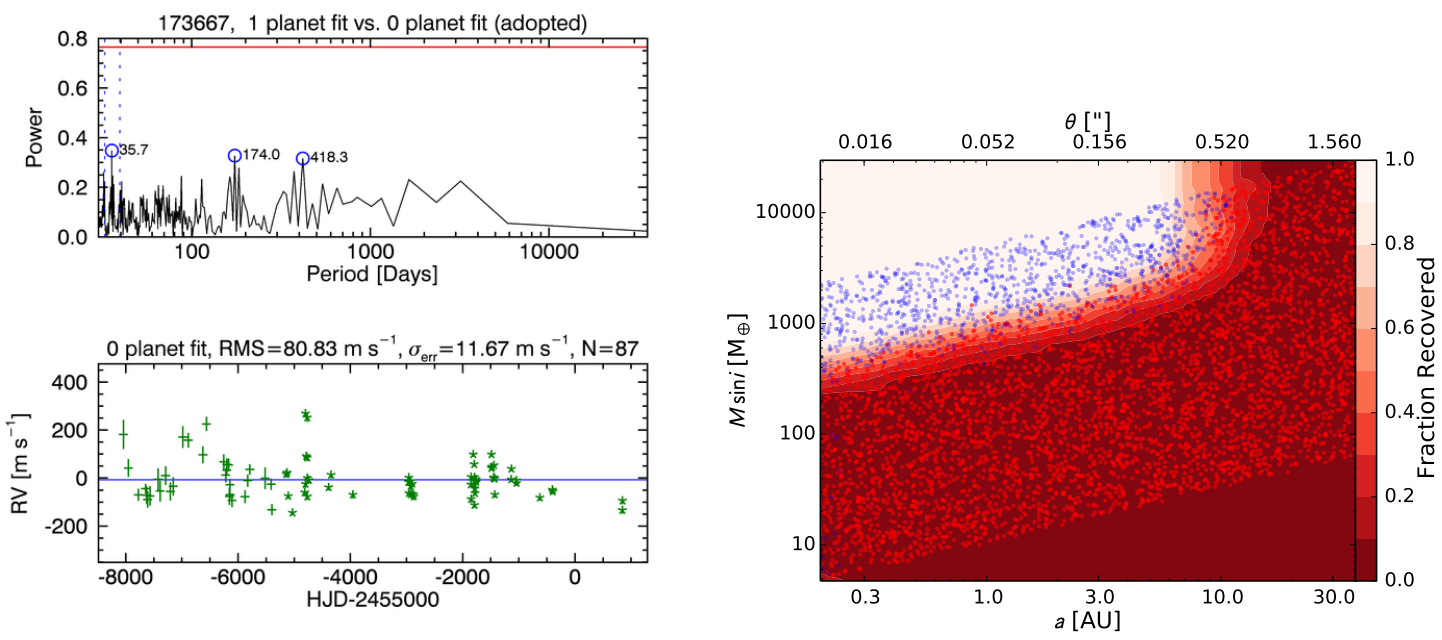

Fig. C.63.- Results from an automated search for planets orbiting the star HD 173667 (HIP 92043; programs = C, A) based on RVs from Lick and/or Keck Observatory. The set of plots on the left (analogous to Figures 4 and 5) show the planet search results and the plot on the right shows the completeness limits (analogous to Fig. 6). See the captions of those figures for detailed descriptions.
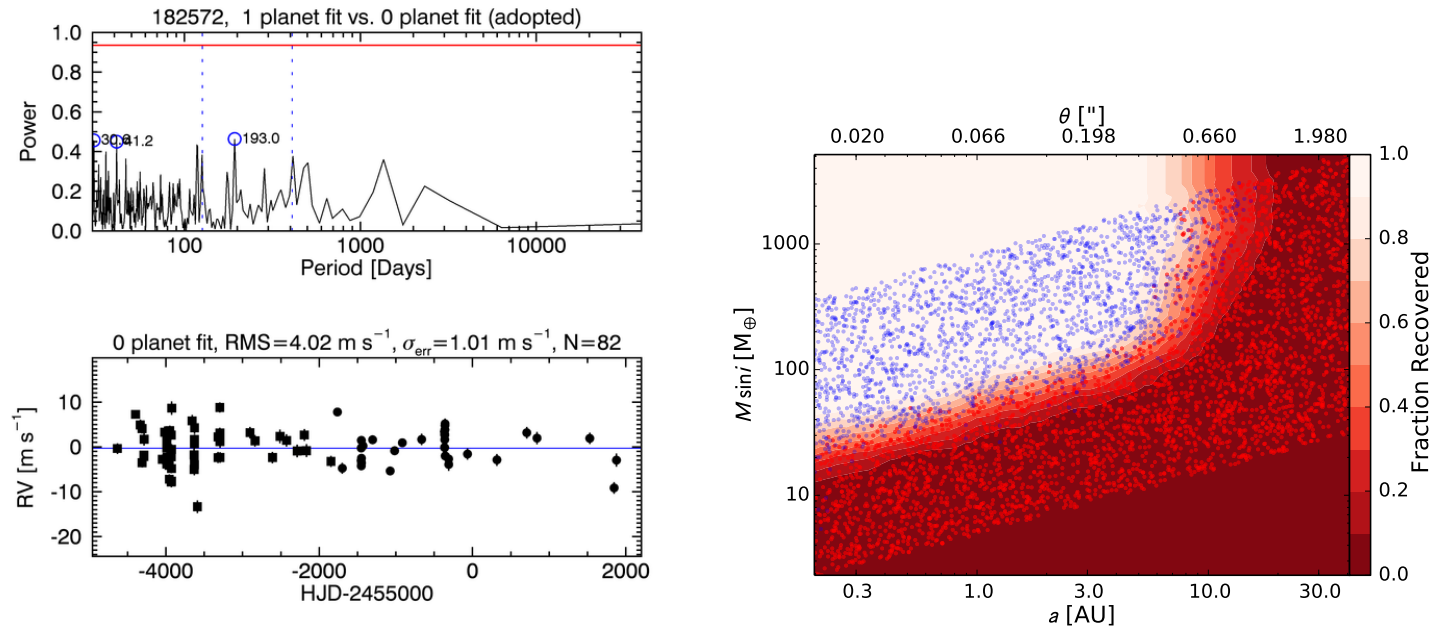

Fig. C.64. - Results from an automated search for planets orbiting the star HD 182572 $($ HIP 95447; programs = S, A) based on RVs from Lick and/or Keck Observatory. The set of plots on the left (analogous to Figures 4 and 5 show the planet search results and the plot on the right shows the completeness limits (analogous to Fig. 6). See the captions of those figures for detailed descriptions. 

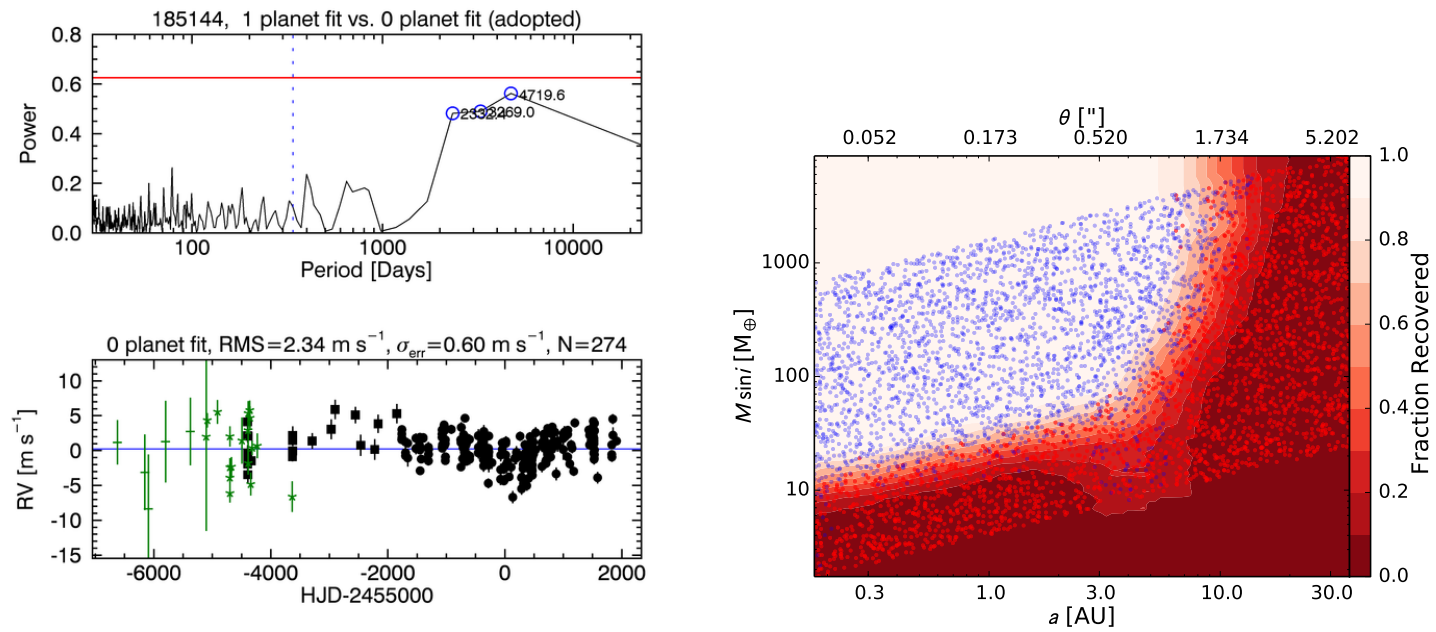

Fig. C.65.- Results from an automated search for planets orbiting the star HD 185144 (HIP 96100; programs = S, C, A) based on RVs from Lick and/or Keck Observatory. The set of plots on the left (analogous to Figures 4 and 5 show the planet search results and the plot on the right shows the completeness limits (analogous to Fig. 6). See the captions of those figures for detailed descriptions. 

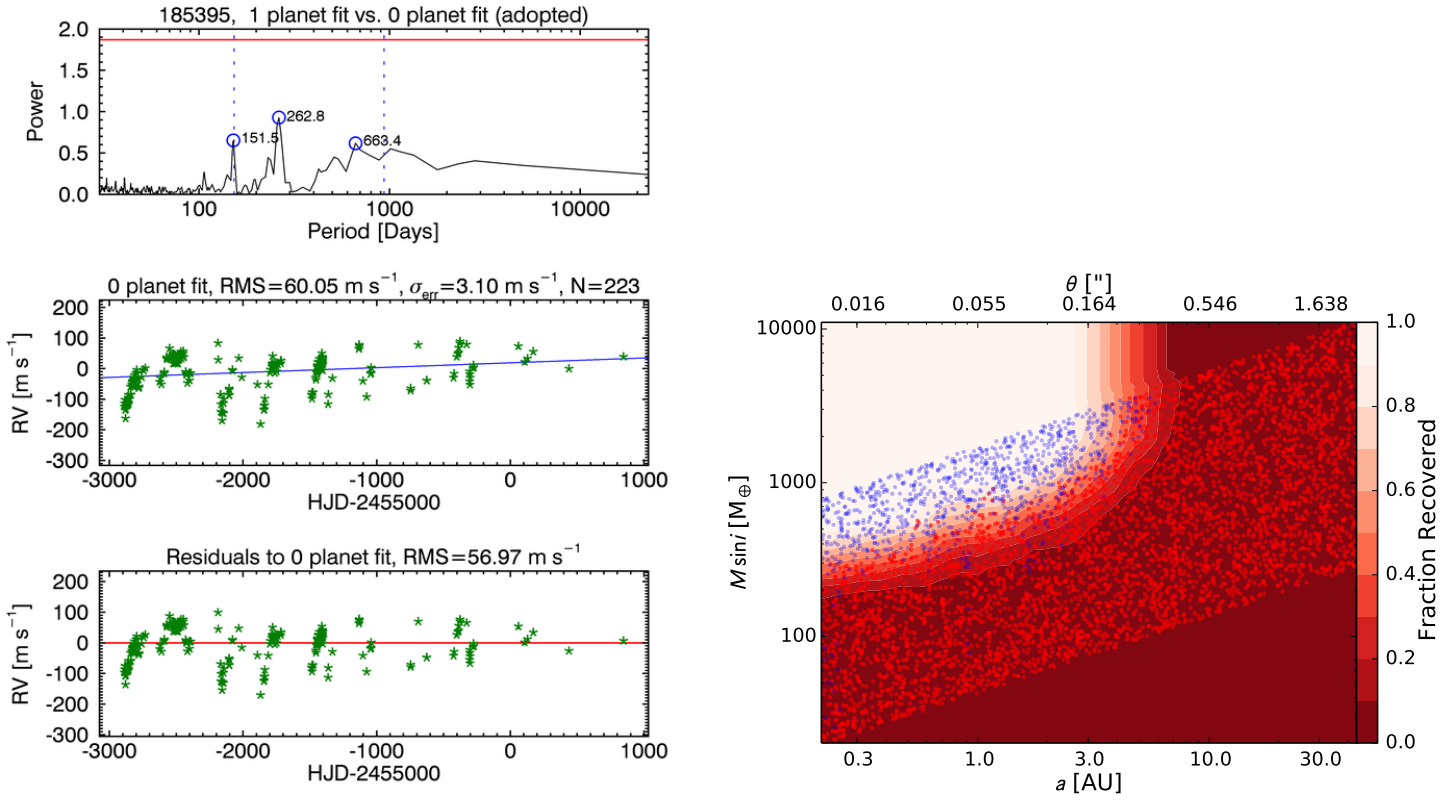

Fig. C.66. - Results from an automated search for planets orbiting the star HD 185395 (HIP 96441; programs = C, A) based on RVs from Lick and/or Keck Observatory. The set of plots on the left (analogous to Figures 4 and 5) show the planet search results and the plot on the right shows the completeness limits (analogous to Fig. 6). See the captions of those figures for detailed descriptions. This early-type star (F4 V) has high jitter and a claimed, controversial planet with $P \approx 150$ days. We see evidence in our Lick data for RV variation at this period and other periods related by the yearly alias. Our pipeline formally adopts a linear trend model for the RVs. 

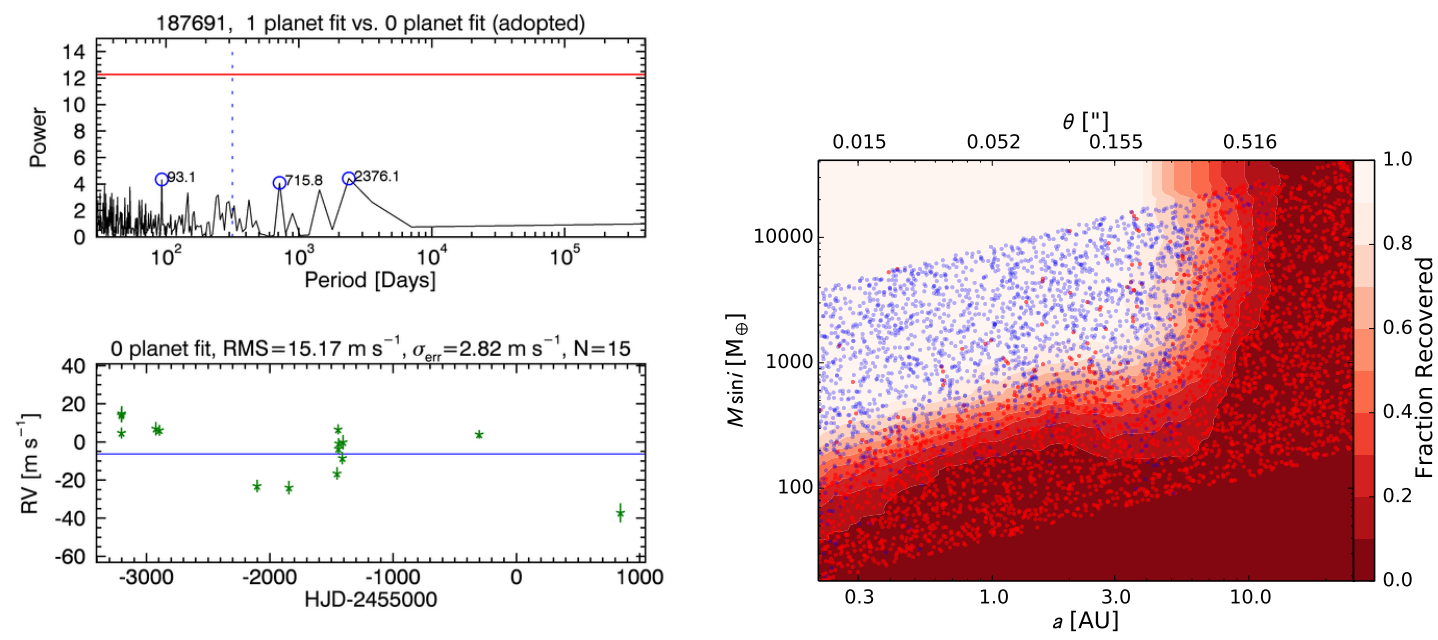

Fig. C.67.- Results from an automated search for planets orbiting the star HD 187691 (HIP 97675; program = A) based on RVs from Lick and/or Keck Observatory. The set of plots on the left (analogous to Figures 4 and 5) show the planet search results and the plot on the right shows the completeness limits (analogous to Fig. 6). See the captions of those figures for detailed descriptions.
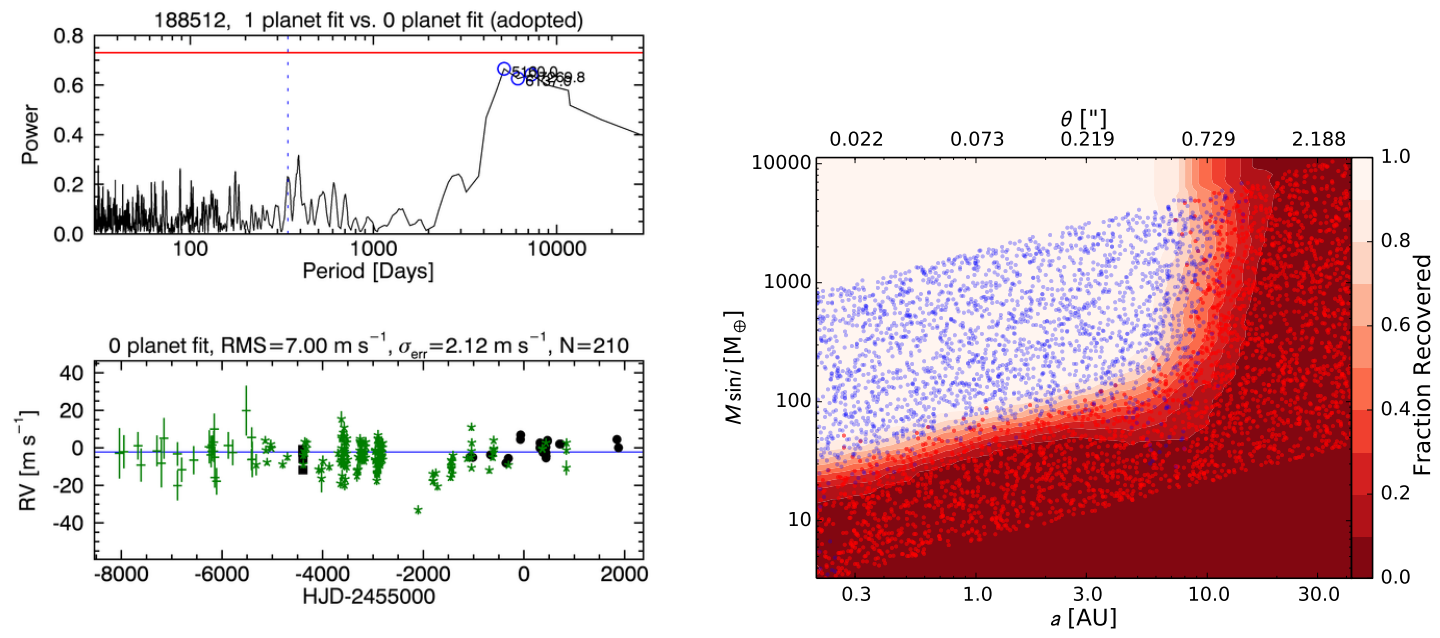

Fig. C.68. - Results from an automated search for planets orbiting the star HD 188512 (HIP 98036; programs = S, C) based on RVs from Lick and/or Keck Observatory. The set of plots on the left (analogous to Figures 4 and 5 show the planet search results and the plot on the right shows the completeness limits (analogous to Fig. 6). See the captions of those figures for detailed descriptions. 

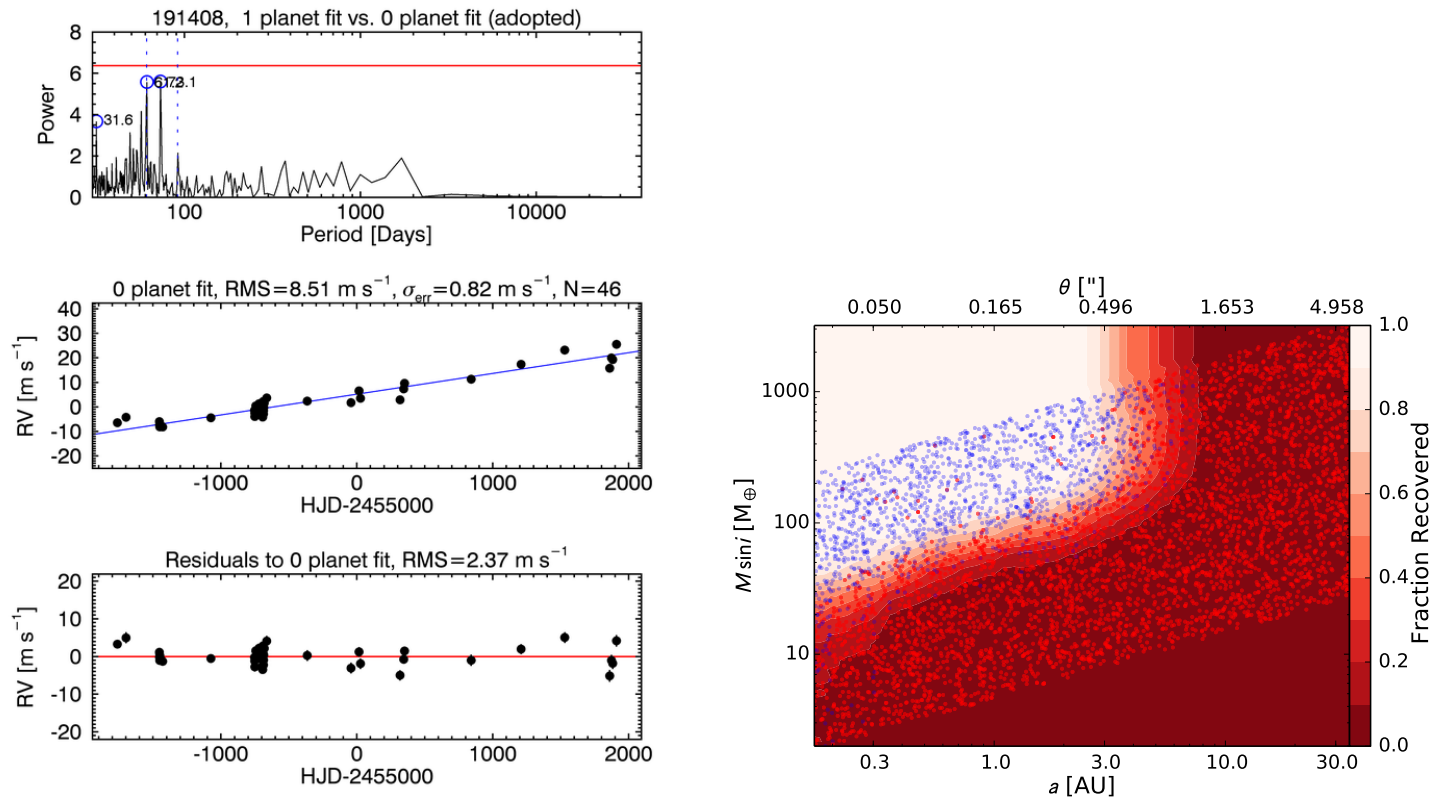

Fig. C.69. - Results from an automated search for planets orbiting the star HD 191408 (HIP 99461; programs = S, C, A) based on RVs from Lick and/or Keck Observatory. The set of plots on the left (analogous to Figures 4 and 5) show the planet search results and the plot on the right shows the completeness limits (analogous to Fig. 6). See the captions of those figures for detailed descriptions. This star shows a slight linear trend with no detectable curvature, presumably due to its common proper motion companion. 

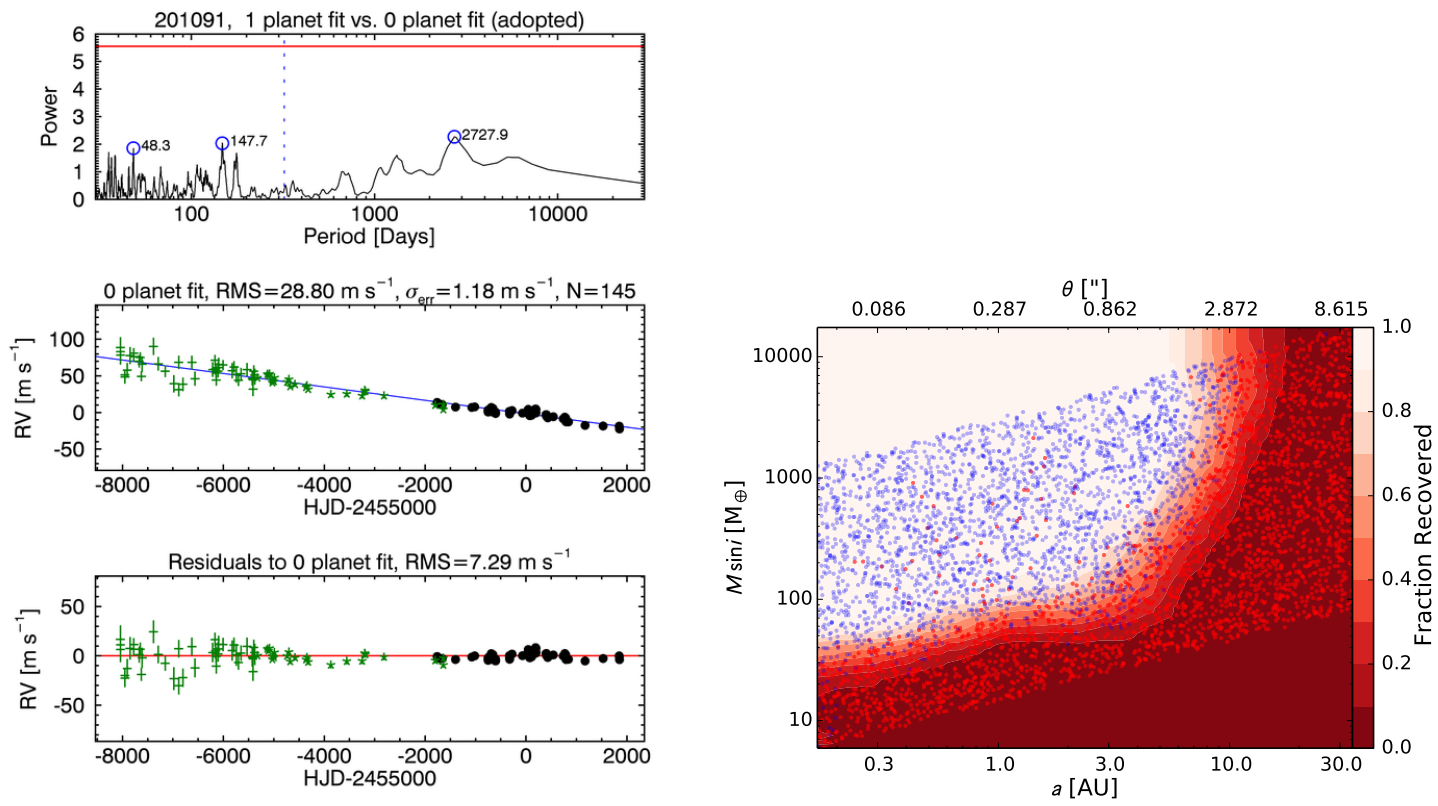

Fig. C.70.- Results from an automated search for planets orbiting the star HD 201091 (HIP 104214; program = S, C, A) based on RVs from Lick and/or Keck Observatory. The set of plots on the left (analogous to Figures 4 and 5 ) show the planet search results and the plot on the right shows the completeness limits (analogous to Fig. 6). See the captions of those figures for detailed descriptions. This star shows a significant linear trend with no detectable curvature, presumably due to its known stellar companion. 

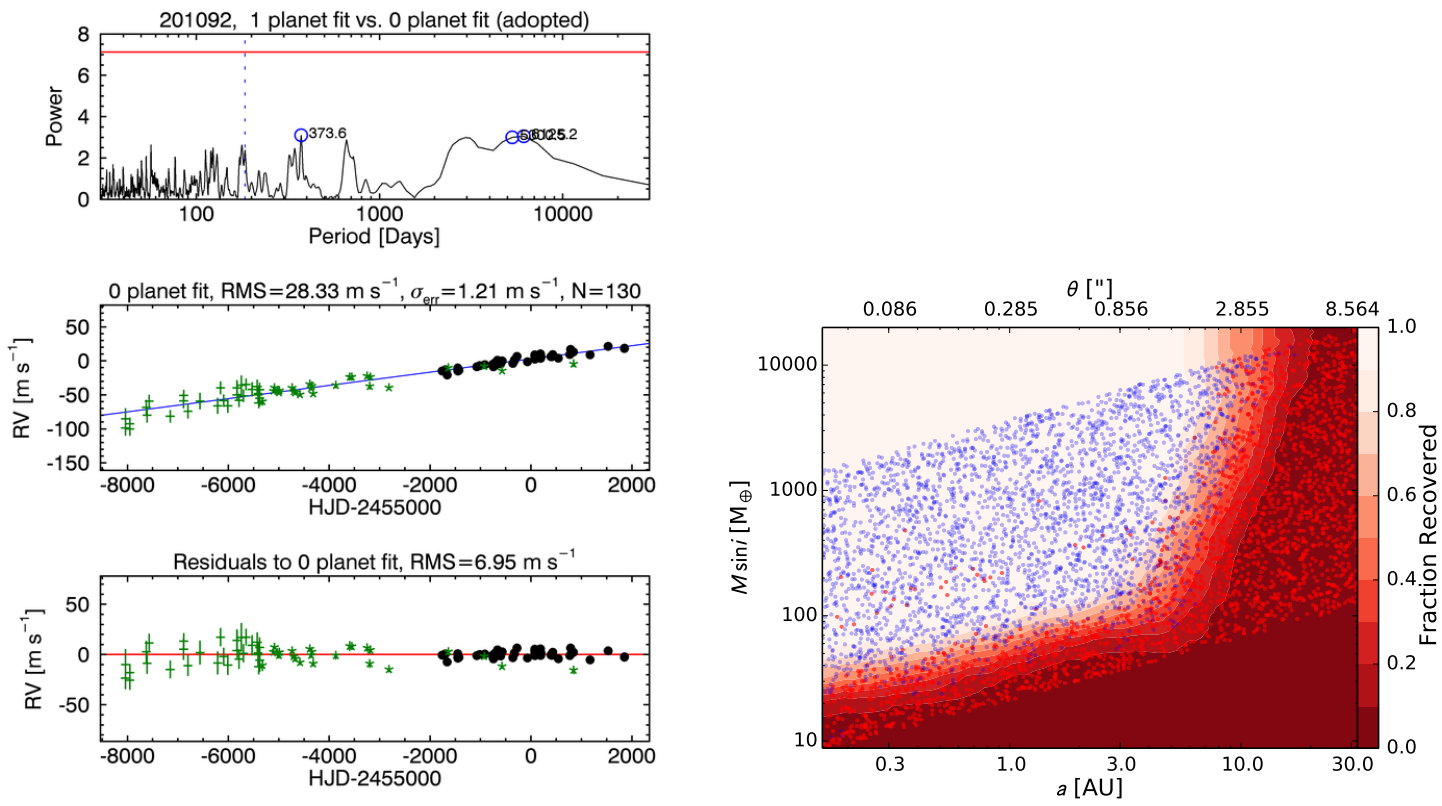

Fig. C.71. - Results from an automated search for planets orbiting the star HD 201092 (HIP 104217; program $=$ S) based on RVs from Lick and/or Keck Observatory. The set of plots on the left (analogous to Figures 4 and 5) show the planet search results and the plot on the right shows the completeness limits (analogous to Fig. 6). See the captions of those figures for detailed descriptions. This star shows a significant linear trend with no detectable curvature, presumably due to its known stellar companion. 

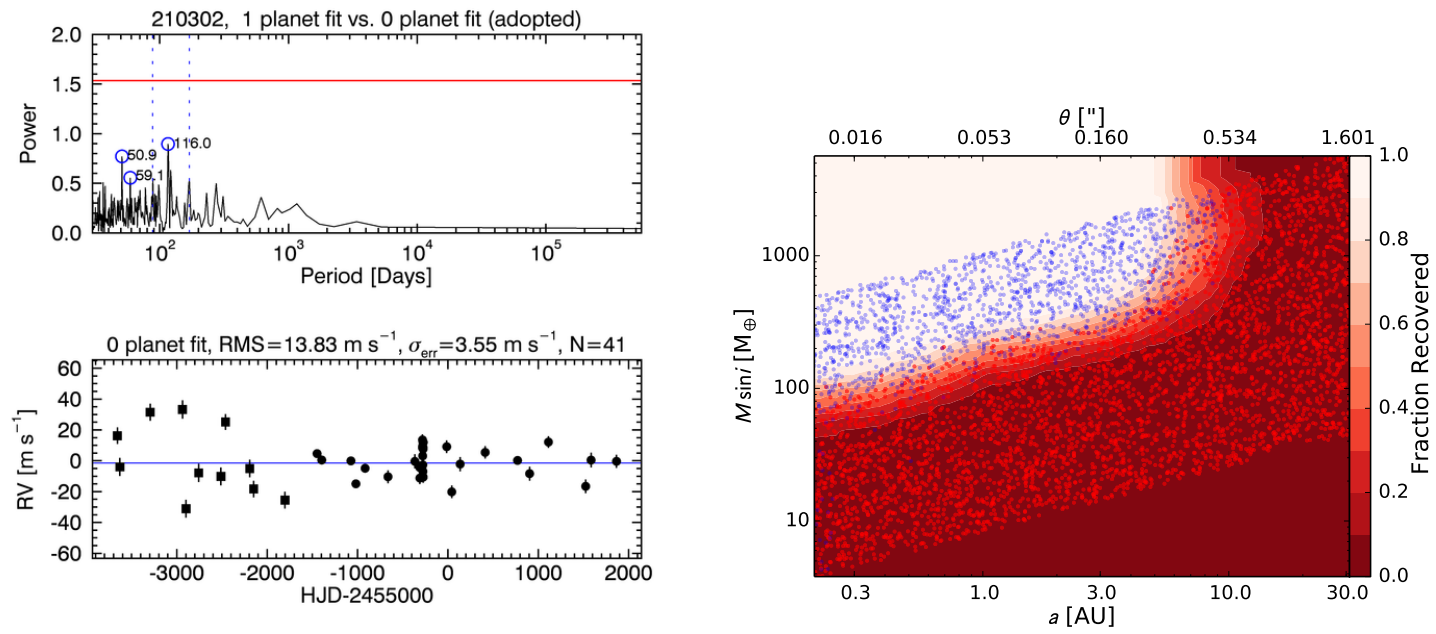

Fig. C.72. - Results from an automated search for planets orbiting the star HD 210302 (HIP 109422; program = A) based on RVs from Lick and/or Keck Observatory. The set of plots on the left (analogous to Figures 4 and 5) show the planet search results and the plot on the right shows the completeness limits (analogous to Fig. 6). See the captions of those figures for detailed descriptions. 

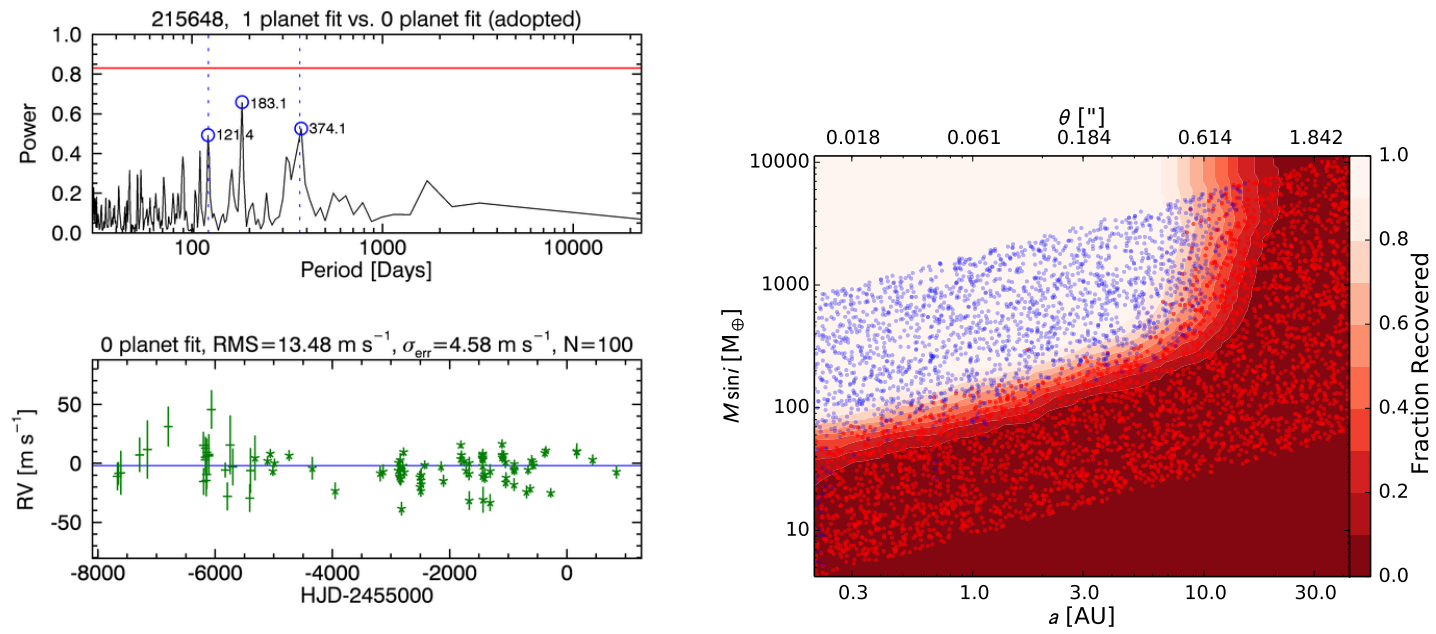

Fig. C.73. - Results from an automated search for planets orbiting the star HD 215648 (HIP 112447; programs = S, C, A) based on RVs from Lick and/or Keck Observatory. The set of plots on the left (analogous to Figures 4 and 5) show the planet search results and the plot on the right shows the completeness limits (analogous to Fig. 6). See the captions of those figures for detailed descriptions. 

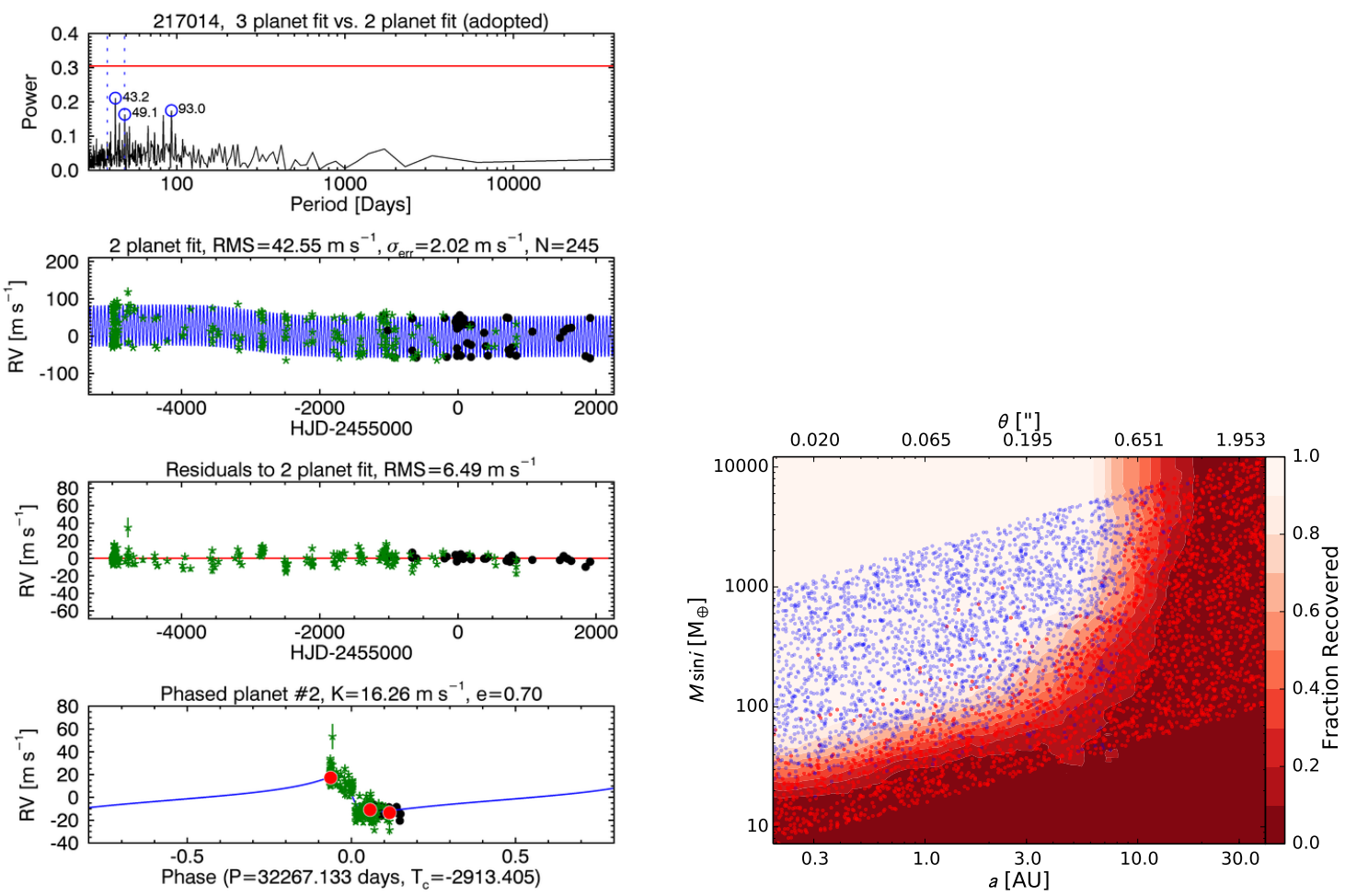

Fig. C.74. - Results from an automated search for planets orbiting the star HD 217014 (HIP 113357; program = S) based on RVs from Lick and/or Keck Observatory. The set of plots on the left (analogous to Figures 4 and 5) show the planet search results and the plot on the right shows the completeness limits (analogous to Fig. 6). See the captions of those figures for detailed descriptions. This star hosts a hot Jupiter. We also detect a long-period signal but this is likely caused by instrumental offsets within the Lick dataset. 

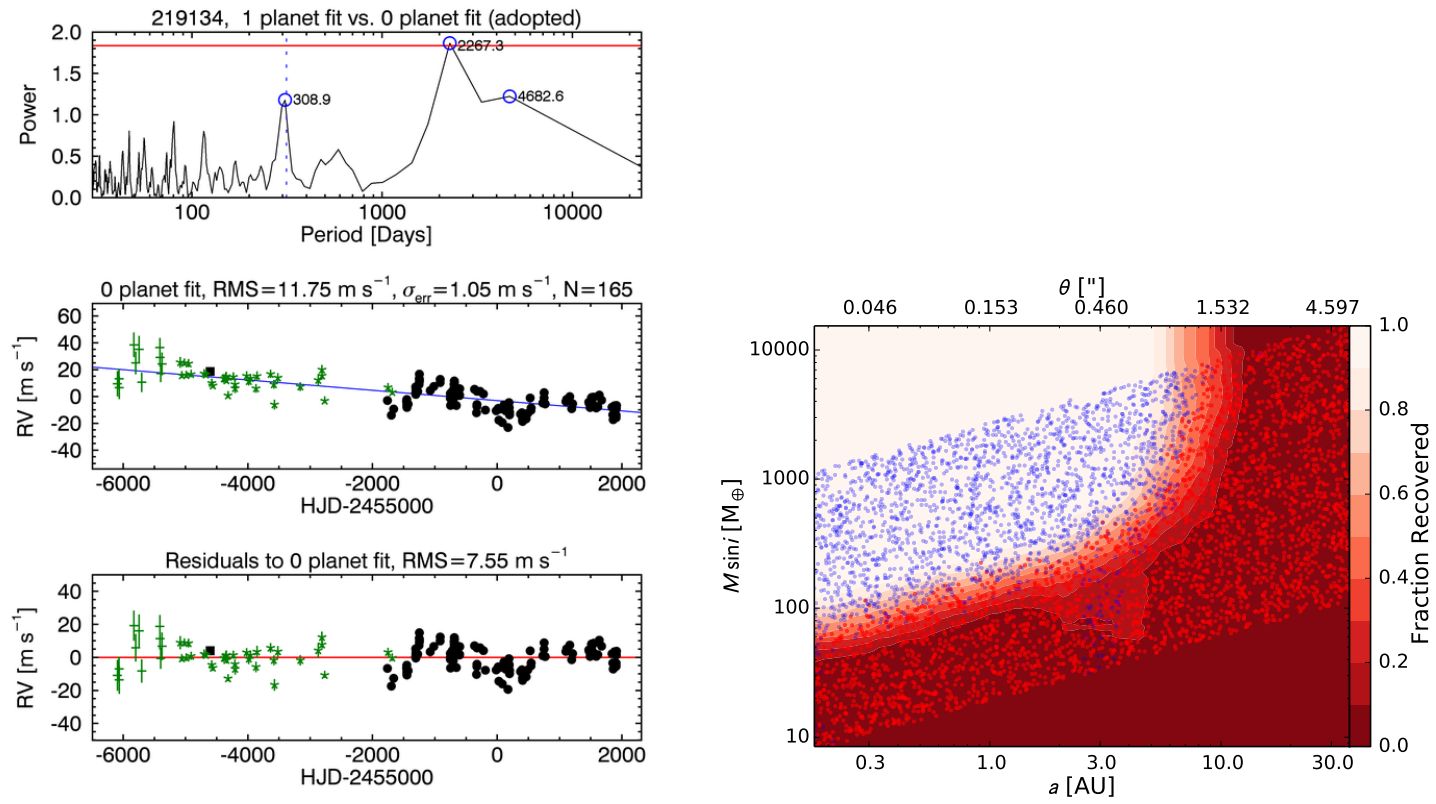

Fig. C.75. - Results from an automated search for planets orbiting the star HD 219134 (HIP 114622; programs = S, C) based on RVs from Lick and/or Keck Observatory. The set of plots on the left (analogous to Figures 4 and 5) show the planet search results and the plot on the right shows the completeness limits (analogous to Fig. 6). See the captions of those figures for detailed descriptions. This star has candidate planets including a giant planet in a 3 AU orbit that we will continue to examine as more RVs are gathered. Our automated pipeline formally prefers a model with a linear trend in the RV time series. 

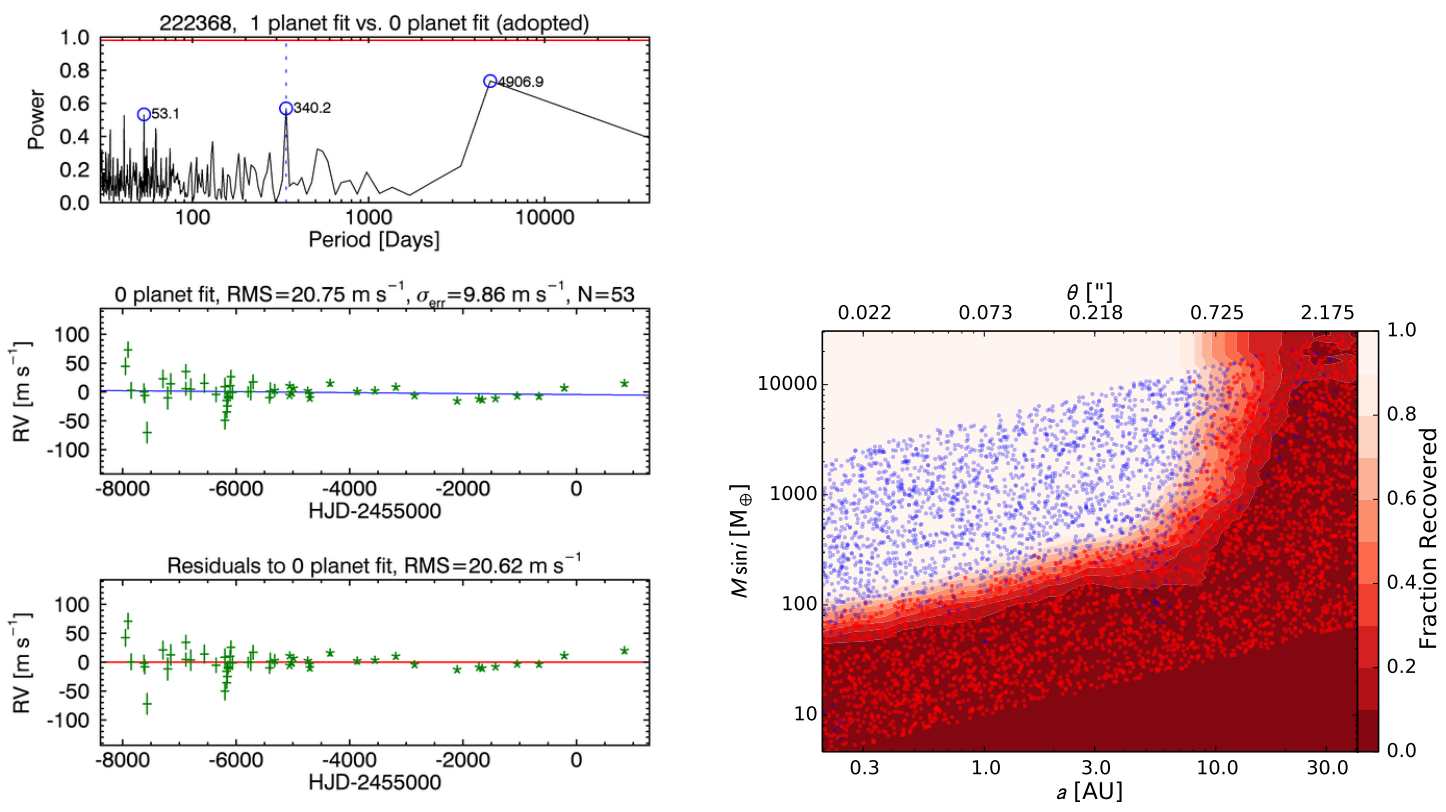

Fig. C.76.- Results from an automated search for planets orbiting the star HD 222368 (HIP 116771; programs = S, C, A) based on RVs from Lick and/or Keck Observatory. The set of plots on the left (analogous to Figures 4 and 5 show the planet search results and the plot on the right shows the completeness limits (analogous to Fig. 6). See the captions of those figures for detailed descriptions. 